\title{
CORIOLIS MASS FLOW RATE METERS \\ FOR LOW FLOWS
}




\section{Promotiecommissie:}

Voorzitter, secretaris

Promotor

Deskundigen

Leden prof.dr.ir. J. van Amerongen, Universiteit Twente

prof.dr.ir. P.P.L. Regtien, Universiteit Twente

ir. J.M. Zwikker, Demcon

ir. W. Jouwsma, Bronkhorst High Tech

prof.dr.ir. S. Stramigioli, Universiteit Twente

prof.dr.ir. C.H. Slump, Universiteit Twente

prof.ir. R.H. Munnig Schmidt, TU Delft

dr.ir. D.M. Brouwer, Universiteit Twente

Coriolis Mass Flow Rate Meters for Low Flows

A. Mehendale

Ph.D Thesis, University of Twente, Enschede, The Netherlands

ISBN: 978-90-365-2727-9

Cover: Toy gyro top. Photograph by Adam Hart-Davis, via http://gallery.hd.org

Rear: A parallel between a gyro and a Coriolis mass-flowmeter

Printing: Print Partners Ipskamp, Enschede, The Netherlands

(C) Aditya Mehendale, 2008 


\title{
CORIOLIS MASS FLOW RATE METERS FOR LOW FLOWS
}

\section{DISSERTATION}

\author{
to obtain \\ the degree of doctor at the University of Twente, \\ on the authority of the rector magnificus, \\ prof.dr. W.H.M. Zijm, \\ on account of the decision of the graduation committee, \\ to be publicly defended \\ on Thursday the $2^{\text {nd }}$ of October, 2008 at 13:15
}

\section{by}

\section{Aditya Mehendale}

born on the $2^{\text {nd }}$ of June 1976

in Pune, India 
This dissertation has been approved by:

Prof.dr.ir. P.P.L. Regtien (promotor) 


\section{Summary}

The accurate and quick measurement of small mass flow rates $(\sim 10 \mathrm{mg} / \mathrm{s})$ of fluids is considered an "enabling technology" in semiconductor, fine-chemical, and food \& drugs industries. Flowmeters based on the Coriolis effect offer the most direct sensing of the mass flow rate, and for this reason do not need complicated translation or linearization tables to compensate for other physical parameters (e.g. density, state, temperature, heat capacity, viscosity, etc.) of the medium that they measure. This also makes Coriolis meters versatile the same instrument can, without need for factory calibration, measure diverse fluid media liquids as well as gases. Additionally, Coriolis meters have a quick response, and can principally afford an all-metal-no-sliding-parts fluid interface.

A Coriolis force is a pseudo-force that is generated when a mass is forced to travel along a straight path in a rotating system. This is apparent in a hurricane on the earth (a rotating system): when air flows towards a low-pressure region from surrounding areas, instead of following a straight path, it "swirls" (in a \{towards + sideways $\}$ motion). The sideways motion component of the swirl may be attributed to the Coriolis (pseudo)force. To harness this force for the purpose of measurement, a rotating tube may be used. The measurand (mass flow rate) is forced through this tube. The Coriolis force will then be observed as a sideways force (counteracting the swirl) acting upon this tube in presence of mass-flows. The Coriolis mass flow meter tube may thus be viewed as an active measurement - a "modulator" where the output (Coriolis force) is proportional to the product of the excitation (angular velocity of the tube) and the measurand (mass flow rate).

From a constructional viewpoint, the Coriolis force in a Coriolis meter is generated in an oscillating (rather than a continuously rotating) meter-tube that carries the measurand fluid. In such a system (typically oscillating at a chosen eigenfrequency of the tube-construction), besides the Coriolis force, there are also inertial, dissipative and spring-forces that act upon the meter tube. As the instrument is scaled down, these other forces become significantly larger than the generated Coriolis force. Several "tricks" can be implemented to isolate these constructional forces from the Coriolis force, based on orthogonality - in the time domain, in eigenmodes and in terms of position (unobservable $\&$ uncontrollable modes, symmetry, etc.).

Being an active measurement, the design of Coriolis flowmeters involves multidisciplinary elements - fluid dynamics, fine-mechanical construction principles, mechanical design of the oscillating tube and surroundings, sensor and actuator design, electronics for driving, sensing and processing and software for data manipulation \& control. This nature lends itself well to a mechatronic system-design approach. Such an approach, combined with a "V-model" system development cycle, aids in the realization of a Coriolis meter for low flows.

Novel concepts and proven design principles are assessed and consciously chosen for implementation for this "active measurement". These include:

shape and form of the meter-tube a statically determined affixation of the tube contactless pure-torque actuator for exciting the tube contactless position-sensing for observing the (effect of) Coriolis force strategic positioning of the sensor \& actuator to minimize actuator crosstalk and to maximize the position sensor ratio-gain

- ratiometric measurement of the (effect of) the Coriolis force to identify the measurand (i.e. the mass flow rate)

- multi-sensor pickoff and processing based solely on time measurement - this is tolerant to component gain mismatch and any drift thereof

- measurement of temperature and correction for its effect of tube-stiffness 
The combined effect of these and other choices is the realization of a fully working prototype. Such prototype devices are presented as a test case in this thesis to assess the effectiveness of these choices.

A "V-model" system-development cycle involves the critical definition of requirements at the beginning and a detailed evaluation at the end to verify that these are met. To reduce ambiguity of intent, several test methods are defined right at the beginning with this model in mind. These end-tests complete the "cycle" - a loop that began with the concepts and with the definition of requirements. However, a V-model also entails shorter iterative cycles that help refine concepts and components during the intermediate design phases. Such "inner loops" are also presented to illustrate design at subsystem and component levels.

A Coriolis flowmeter prototype with an all-steel fluid-interface is demonstrated, that has a specified full-scale ("FS") mass flow rate of $200 \mathrm{~g} / \mathrm{h}(\sim 55 \mathrm{mg} / \mathrm{s})$ of water. This instrument has a long-term zero-stability better than $0.1 \% \mathrm{FS}$ and sensitivity stability better than $0.1 \%$, density independence of sensitivity (within $0.2 \%$ for liquids), negligible temperature effect on drift \& sensitivity, and a $98 \%$ settling time of less than 0.1 seconds. For higher and/or negative pressure drops, these instruments have been seen to operate from -50xFS to $+50 \times \mathrm{FS}$ (i.e. from $-10 \mathrm{~kg} / \mathrm{h}$ to $+10 \mathrm{~kg} / \mathrm{h}$ ) without performance degradation - particularly important in order to tolerate flow-pulsations in dosing applications.

Finally, the results of the present work are discussed, and recommendations are made for possible future research that would add to it. Two important recommendations are made about the possibility to seek, by means of an automated optimization algorithm, an improved tube shape for sensing the flow, and about constructional improvements to make the measuring instrument more robust against external vibrations. 


\section{Samenvatting}

De nauwkeurige en snelle meting van kleine massastromen $(\sim 10 \mathrm{mg} / \mathrm{s})$ wordt in de halfgeleider-, fijnchemische, voedingsmiddelen- en medische industrie als een technologie gezien welke vernieuwende processen mogelijk maken. De op het Coriolis-effect gebaseerde massastroommeters meten direct de massastroom, dus zonder omrekeningen om andere fysische parameters (bijvoorbeeld dichtheid, aggregratietoestand, temperatuur, warmtecapaciteit, viscositeit, enz.) van de te meten grootheid te compenseren. Hierdoor zijn deze instrumenten veelzijdig; hetzelfde instrument kan, zonder noodzaak van mediumspecifieke kalibratie, diverse vloeistoffen en gassen meten. Bovendien hebben deze meters een korte reactietijd en het vloeistofcontact kan volledig in RVS uitgevoerd worden, zonder glijdende delen.

De Coriolis-kracht is een pseudo-kracht die optreedt wanneer een massa in een roterend systeem loodrecht op de rotatierichting beweegt. Dit treedt bijvoorbeeld op in een orkaan wanneer op de draaiende aarde de lucht die naar een lage druk regio stroomt, begint af te buigen en daardoor de bekende spiraalvorm krijgt. Deze lucht ondervindt een zijwaartse pseudo-kracht die veroorzaakt wordt door het Coriolis-effect. Om deze kracht te kunnen beheersen en daarmee te meten, kan een roterende buis worden gebruikt. De te meten vloeistof (of het te meten gas) wordt gedwongen door deze buis te stromen. De Corioliskracht kan nu worden waargenomen als een kracht die afbuiging van deze stromende vloeistof tegenwerkt. De Coriolis-buis van de massastroommeter kan worden beschouwd als een actief meetprincipe, ofwel modulator, waarbij de Coriolis-kracht evenredig is aan het product van de excitatie (hoeksnelheid van de buis) en de massastroomsnelheid.

De benodigde hoeksnelheid van de vloeistofdragende buis wordt gerealiseerd door een oscillerende (in plaats van continu roterende, zoals de aarde) beweging; dit om glijdende delen te vermijden. De oscillatie van de buis vindt plaats op een eigenfrequentie van de buisconstructie. Door deze oscillatie ontstaan krachten tengevolge van inertiële, verende en dempende eigenschappen van de buisconstructie. Naarmate de meetbuis verkleind (geschaald) wordt, worden deze krachten vele malen groter ten opzichte van de Corioliskracht. Verschillende "trucs" kunnen gebruikt worden om deze krachten afkomstig van de constructie te isoleren van de Coriolis-kracht. Deze "trucs" zijn gebaseerd op orthogonaliteit zowel in het tijddomein als met betrekking tot eigenmodes en de positionering van componenten.

Juist omdat het een actief meetprincipe is, is het ontwerp van een Coriolis-massastroommeter multidisciplinair. Vloeistofdynamica, fijnmechanische en constructieprincipes, elektronica voor de aansturing, sensoren, signaalbewerking en informatica voor regelen en sturen, spelen een rol in de prestaties van het uiteindelijke meetinstrument. Daarom is dit ontwerp geschikt voor een mechatronische ontwerp-aanpak. Deze aanpak heeft er samen met een systeemontwerpcyclus volgens het " $V$-model" aan bijgedragen om een voor zeer lage massastromen bedoelde Coriolis-massastroommeter te realiseren.

Zowel nieuwe concepten als bewezen ontwerpregels zijn overwogen en steeds is een bewuste keuze gemaakt bij aspecten van de implementatie van de actieve meting:

Vorm van de meetbuis

- Statisch bepaalde buisfixatie en omgevingsconstructie

- Contactloze 'zuiver-koppel' aanstoting van de buis

- Contactloos meetprincipe om het effect van de Coriolis-kracht te meten

- Strategische positionering van de actuator en sensoren om de overspraak van de actuatiekracht naar de Coriolis-beweging te minimaliseren en tegelijk de Coriolisbeweging zo groot mogelijk te maken ten opzichte van de actuatiebeweging

- Ratiometrische bepaling van de Coriolis-kracht op de buis om de massastroom te ijken 
- Opname door middel van meerdere, uitsluitend op tijdmeting gebaseerde sensoren, waardoor de opname ongevoelig is voor ongelijke signaalamplitudes of veranderingen daarvan.

- Meten van een correctie voor de door temperatuur beïnvloede eigenschappen van het buismateriaal

Bovenstaande concepten hebben tezamen bijgedragen aan het realiseren van volledig werkende prototypes. Deze prototypes zijn onderworpen aan een aantal testen om de doeltreffendheid van de gemaakte keuzes aan te tonen.

De systeemontwerpcyclus volgens het "V-model" eist het kritisch vastleggen van systeemeisen (requirements) tijdens de beginfase en een evaluatie in de eindfase om aan te tonen dat de eisen gehaald zijn. Om onduidelijkheid met betrekking tot meetmethoden te voorkomen, moeten deze tijdens de beginfase vastgelegd worden. De eindmetingen voltooien de lus die begon met het vastleggen van de systeemeisen. Binnen het V-model kunnen ook meerdere kleinere iteratieve lussen gemaakt worden gedurende de systeemontwikkeling om tussendoor de concepten te verbeteren. Naast de grotere lus zijn in dit verslag ook enkele kleinere lussen gepresenteerd om het subsysteem en het ontwerp op componentniveau toe te lichten.

Een Coriolis-massastroommeter met een "Full Scale" (FS) bereik van $200 \mathrm{~g} / \mathrm{uur}$ water wordt in deze thesis beschreven. Deze meter heeft een nulpunts-stabiliteit beter dan $0.1 \%$ FS en een gevoeligheidsverandering kleiner dan $0.1 \%$. De invloed van de vloeistofdichtheid op de gevoeligheid leidt tot een fout kleiner dan $0.2 \%$. De invloed van de temperatuur op de gevoeligheid en het nulpuntsverloop is verwaarloosbaar klein. Verder is de reactietijd, gedefinieerd als de benodigde tijd om $98 \%$ van de eindwaarde door te geven, minder dan 0.1 seconden.

Tot slot worden de resultaten van het huidige onderzoek besproken en zijn er aanbevelingen gedaan voor mogelijk vervolgonderzoek. Twee belangrijke aanbevelingen zijn gedaan, namelijk om door een geautomatiseerd optimalisatie-algoritme een verbeterde buisvorm voor het meten van de flow te ontdekken en om door constructieve verbeteringen de meetbuisvorm ongevoelig te maken voor externe trillingen. 


\section{Listing of symbols}

\begin{tabular}{|c|c|c|}
\hline Symbol(s) & Meaning (unless specified otherwise) & Unit \\
\hline $\bar{a}$ & Acceleration vector & {$\left[\mathrm{m} \cdot \mathrm{s}^{-2}\right]$} \\
\hline$d$ & Deflection & {$[\mathrm{m}]$} \\
\hline$f$ & Frequency & {$[\mathrm{Hz}]\left(\left[\mathrm{s}^{-1}\right]\right)$} \\
\hline$h$ & Height & {$[\mathrm{m}]$} \\
\hline$k$ & Stiffness, linear or angular & $\begin{array}{l}{\left[\mathrm{N} \cdot \mathrm{m}^{-1}\right] \text { or }} \\
{\left[\mathrm{N} \cdot \mathrm{m} \cdot \mathrm{rad}^{-1}\right]}\end{array}$ \\
\hline$k_{m}$ & Motor constant & {$[\mathrm{N} \cdot \mathrm{m} / \mathrm{A}]$} \\
\hline$l$ & Length & {$[\mathrm{m}]$} \\
\hline$m$ & Mass & {$[\mathrm{kg}]$} \\
\hline$r$ & Radius (see context) & {$[\mathrm{m}]$} \\
\hline $\bar{r}$ & Position vector (referred to origin) & {$[\mathrm{m}]$} \\
\hline$t$ & Time & {$[\mathrm{s}]$} \\
\hline $\bar{v}$ & Velocity vector & {$\left[\mathrm{m} \cdot \mathrm{s}^{-1}\right]$} \\
\hline$A$ & (Cross sectional) area & {$\left[\mathrm{m}^{2}\right]$} \\
\hline $\bar{B}$ & Magnetic flux density vector & {$[\mathrm{T}]$} \\
\hline$E_{x}$ & Elastic modulus of a material $x$ & {$\left[\mathrm{~N} \cdot \mathrm{m}^{-2}\right]$} \\
\hline $\bar{F}$ & Force vector & {$[\mathrm{N}]$} \\
\hline$I_{o}$ & Area moment of inertia & {$\left[\mathrm{m}^{4}\right]$} \\
\hline$I D$ & Inner diameter (of tube) & {$[\mathrm{m}]$} \\
\hline$L$ & Total length (of the Coriolis tube) & {$[\mathrm{m}]$} \\
\hline$O D$ & Outer diameter (of tube) & {$[\mathrm{m}]$} \\
\hline$P$ & Pressure & {$\left[\mathrm{kg} \cdot \mathrm{m}^{-1} \cdot \mathrm{s}^{-2}\right]$} \\
\hline$R$ & Resistance or damping (see context) & {$[\Omega]$ or $[\mathrm{N} \cdot \mathrm{s} / \mathrm{m}]$} \\
\hline$R n$ & Reynolds number & {$[-]$} \\
\hline$T$ & Temperature & $\begin{array}{l}{\left[{ }^{\circ} \mathrm{C}\right] \text { or }[\mathrm{K}] \text {, }} \\
\text { as specified }\end{array}$ \\
\hline$\eta$ & Dynamic viscosity & {$\left[\mathrm{kg} \cdot \mathrm{m}^{-1} \cdot \mathrm{s}^{-1}\right]$} \\
\hline$\theta$ & $\begin{array}{l}\text { Angular deflection (rotational stance) of Coriolis tube, usually oscillatory } \\
\text { and due to excitation }\end{array}$ & radian $[-]$ \\
\hline$\dot{\theta}$ & $\begin{array}{l}\text { Angular velocity (rate of chance of angular deflection) of tube, usually } \\
\text { oscillatory and due to excitation }\end{array}$ & radian per second $\left[\mathrm{s}^{-1}\right]$ \\
\hline$\rho$ & Density or electrical resistivity, as per context & {$\left[\mathrm{kg} \cdot \mathrm{m}^{-3}\right]$ or $[\Omega \cdot \mathrm{m}]$} \\
\hline$\sigma$ & Stress & {$\left[\mathrm{N} \cdot \mathrm{m}^{-2}\right]\left(\left[\mathrm{kg} \cdot \mathrm{m}^{-1} \cdot \mathrm{s}^{-2}\right]\right)$} \\
\hline$\omega$ & Oscillatory angular frequency (rate of change of phase of oscillation) & radian per second $\left[\mathrm{s}^{-1}\right]$ \\
\hline $\bar{\omega}$ & Angular velocity (only in 2.1 ) & radian per second $\left[\mathrm{s}^{-1}\right]$ \\
\hline$\Delta P$ & Pressure drop (across a tube) & {$\left[\mathrm{N} \cdot \mathrm{m}^{-2}\right]\left(\left[\mathrm{kg} \cdot \mathrm{m}^{-1} \cdot \mathrm{s}^{-2}\right]\right)$} \\
\hline $\mathrm{T}$ & Torque & {$[N \cdot \mathrm{m}]$} \\
\hline$\Phi ; \Phi_{m}$ & Mass flow rate & {$\left[\mathrm{kg} \cdot \mathrm{s}^{-1}\right]$ or $[\mathrm{g} / \mathrm{h}]$} \\
\hline$\Phi_{v}$ & Volume flow rate & {$\left[\mathrm{m}^{3} \cdot \mathrm{s}^{-1}\right]$} \\
\hline$\psi_{n}$ & $\begin{array}{l}\text { Phase of the signal (phasor) 'n', WRT some (specified or arbitrary) } \\
\text { reference }\end{array}$ & radian $[-]$ \\
\hline$\psi_{1-2}$ & $\begin{array}{l}\text { Phasor angle (fundamental mode phase difference) between signals } 1 \\
\text { and } 2 ; \psi_{1-2}=\psi_{2}-\psi_{1}\end{array}$ & radian $[-]$ \\
\hline
\end{tabular}




\begin{tabular}{|c|l|}
\hline \multicolumn{2}{|l|}{ Suffixes and accents } \\
\hline$\left.[\cdots]_{c} ; \cdots\right]_{c o r}$ & Caused by Coriolis force \\
\hline$[\cdots]_{t w}$ & Related to 'twist' motion \\
\hline$[\cdots]_{s w}$ & Related to 'swing' motion \\
\hline$[\cdots]_{e i g}$ & Related to eigenfrequency \\
\hline$[\cdots]_{i n}$ & Viewed in an inertial coordinate frame \\
\hline$[\cdots]_{r o t}$ & Viewed in a rotating coordinate frame \\
\hline$[\cdots]_{o s c}$ & Related to oscillatory motion \\
\hline$[\cdots \cdot]$ & Signifying oscillatory nature \\
\hline$\langle\cdots\rangle$ & Mean value \\
\hline
\end{tabular}

\begin{tabular}{|c|c|}
\hline \multicolumn{2}{|c|}{ Acronyms \& short-forms } \\
\hline COTS & Common (or Commercial) off-the-shelf \\
\hline DUT & Device under test \\
\hline FEM & Finite element method (of computing properties of mechanical constructions) \\
\hline FIR & Finite impulse response (filter) - a type of digital filter with a bucket-brigade topology \\
\hline FR & Functional requirement \\
\hline FS & Full-scale \\
\hline $\mathrm{g} / \mathrm{h}$ & Gram per hour (mass flow rate) - the same as $1 / 3600$ gram per second \\
\hline IC & Integrated circuit \\
\hline ID & Inside diameter (usually of a tube) \\
\hline IIR & Infinite impulse response (filter) - a digital filter with states and with auto-feedback \\
\hline LPF & Low-pass filter - a filter that stops high-frequencies while allowing low frequency signals through \\
\hline MFR & Mass flow rate \\
\hline MI & Moment of inertia \\
\hline OD & Outside diameter (usually of a tube) \\
\hline OEM & $\begin{array}{l}\text { Original Equipment Manufacturer - } A \text { manufacturer }(Y) \text { that incorporates one or more units of } \\
\text { "product- } X^{\prime \prime} \text { (supplied by manufacturer } X \text { ) into its own "product- } Y \text { ". }\end{array}$ \\
\hline PLL & Phase-locked loop - a technique to exactly track the frequency of an cyclic signal using its phase \\
\hline PoP & Proof of principle \\
\hline rad & Radian (dimensionless, hence often omitted, but usually very insightful for the reader) \\
\hline RMS & Root mean square \\
\hline SNR & Signal to noise ratio - a metric for the quality of a measurement \\
\hline SS & Stainless steel \\
\hline TR & Technical requirement \\
\hline
\end{tabular}




\section{Contents}

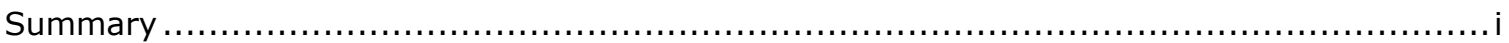

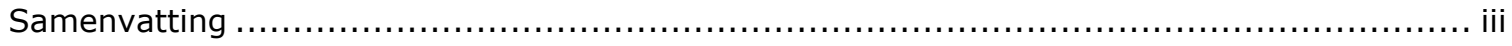

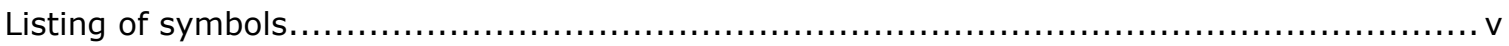

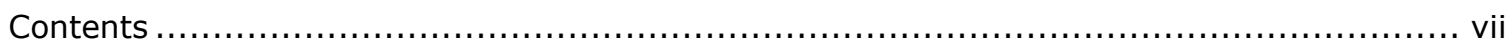

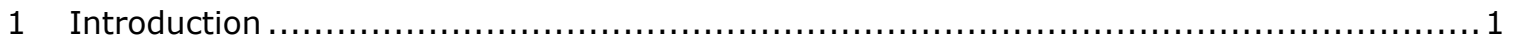

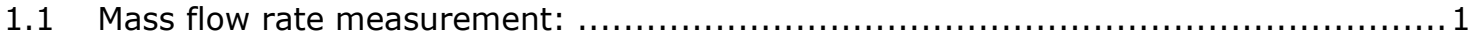

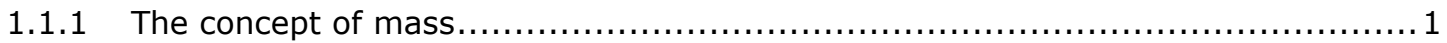

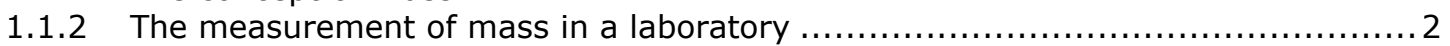

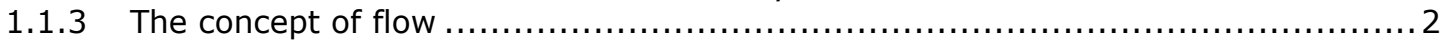

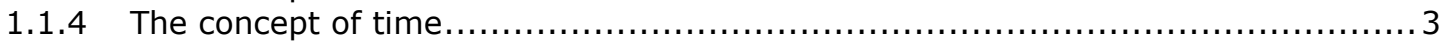

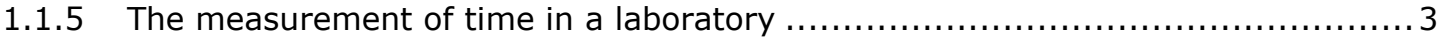

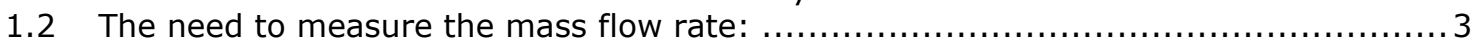

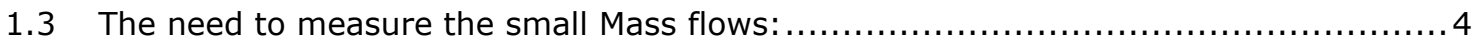

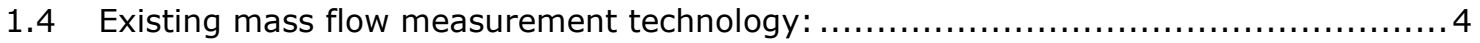

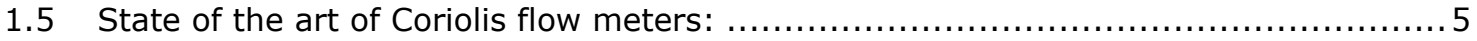

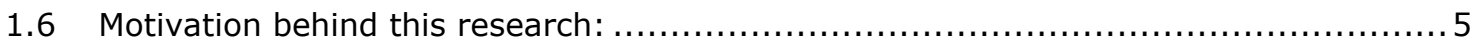

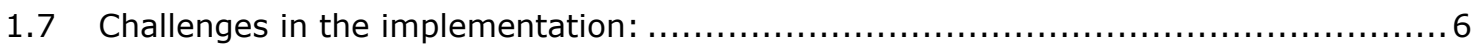

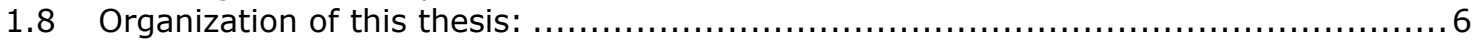

2 Coriolis meters: Current performance and achieved improvements....................... 9

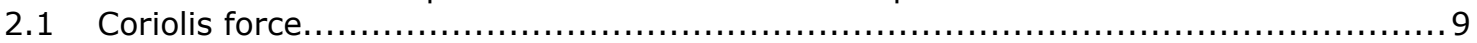

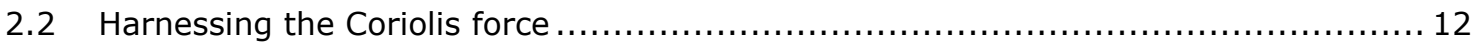

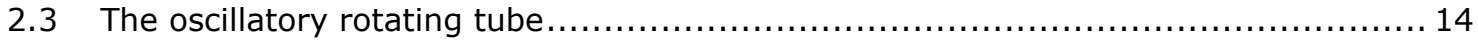

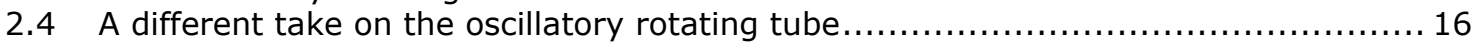

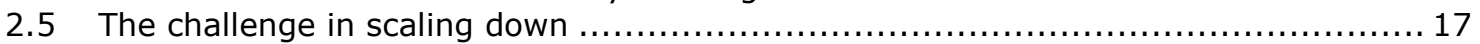

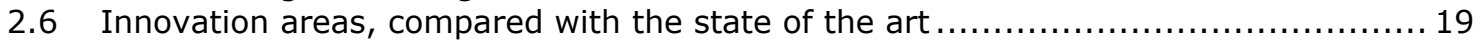

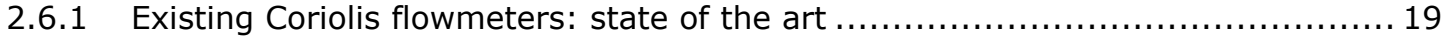

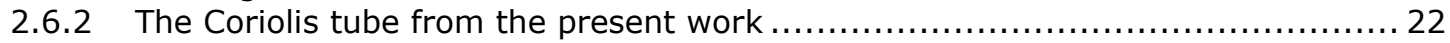

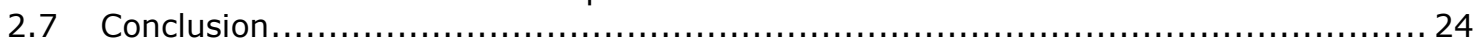

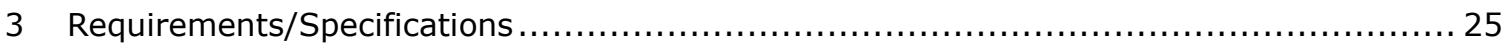

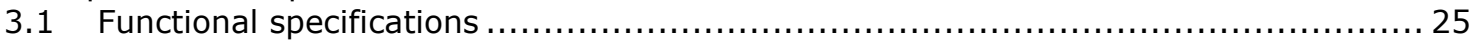

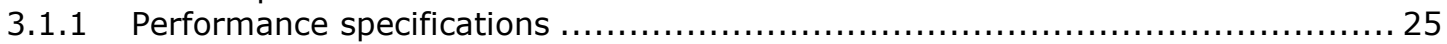

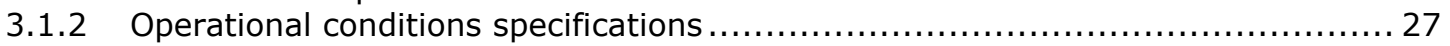

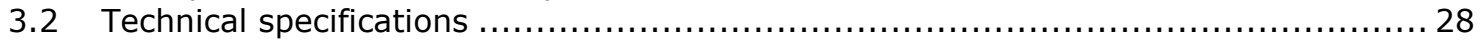

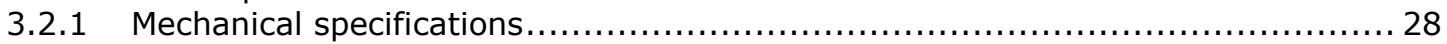

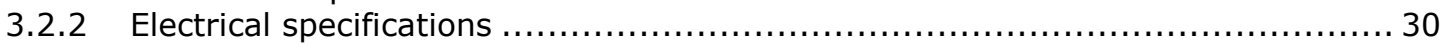

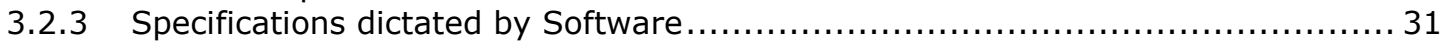

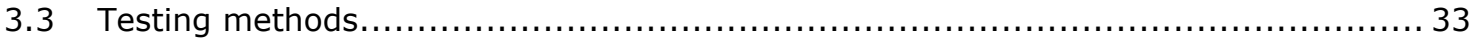

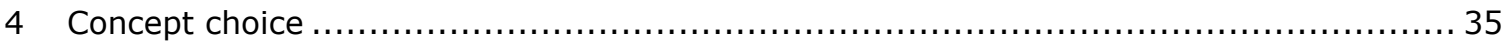

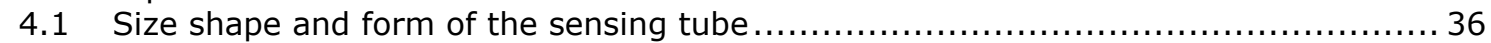

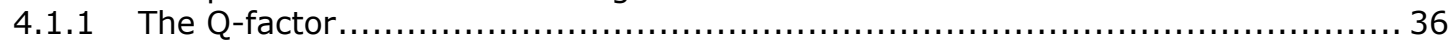

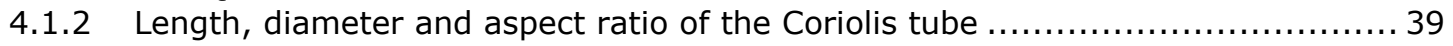

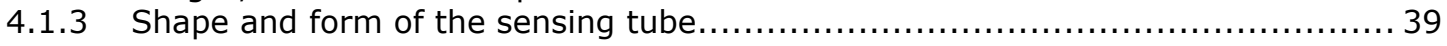

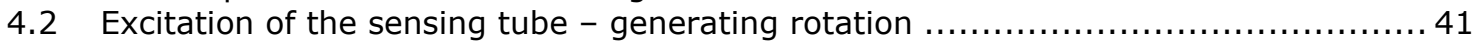

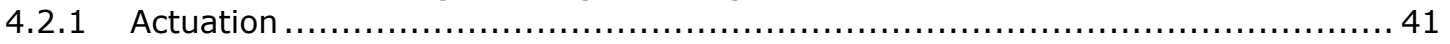

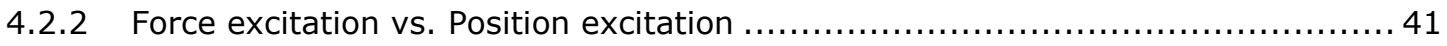

4.2 .3 Possibility of contact-less excitation .................................................. 42

4.2.4 Concept (Part 1) - Lorentz actuator; tube carries current.......................... 44 
4.2.5 Concept (Part 2) - Galvanic isolation of excitation current......................... 44

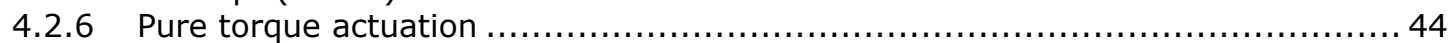

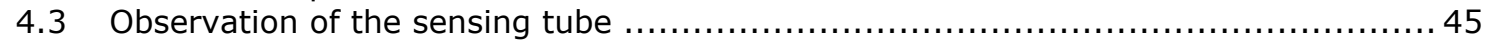

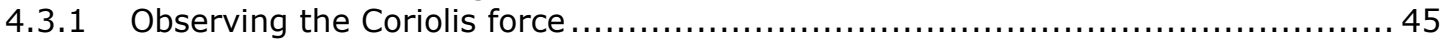

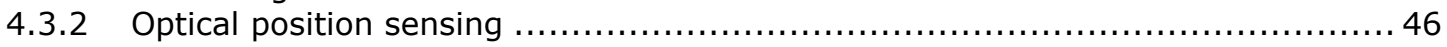

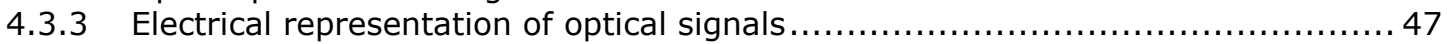

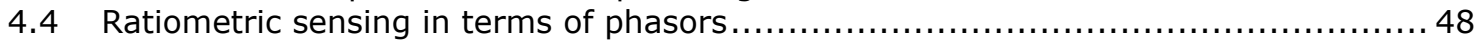

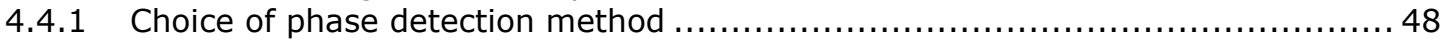

4.4.2 Overview - Dual quadrature demodulation ....................................... 49

4.5 Digital representation of electrical signals (and vice versa) ........................... 50

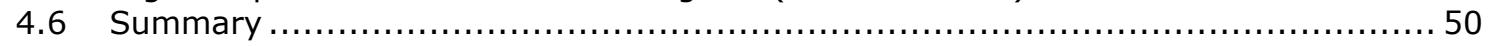

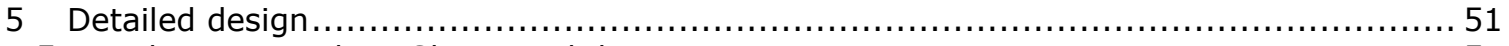

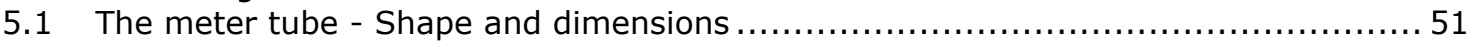

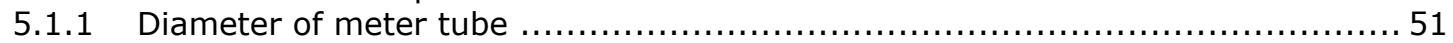

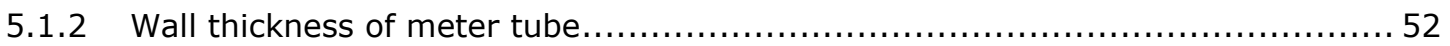

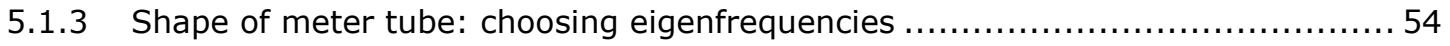

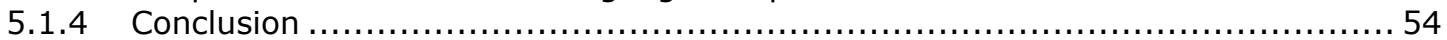

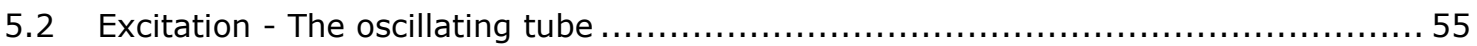

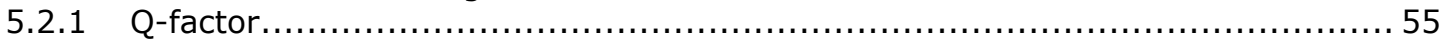

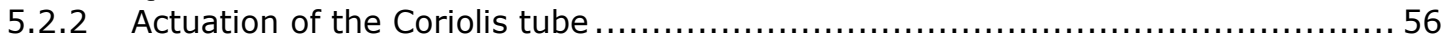

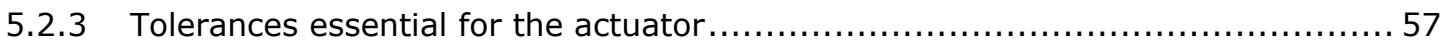

5.2.4 Temperature dependent variation of (stainless steel) material properties ...........59

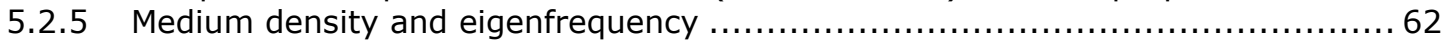

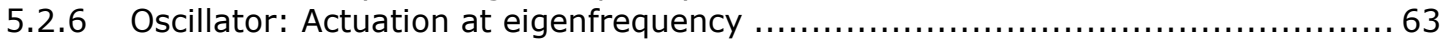

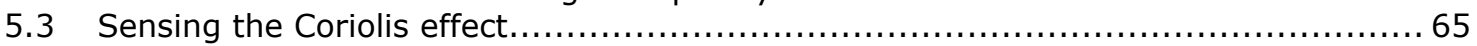

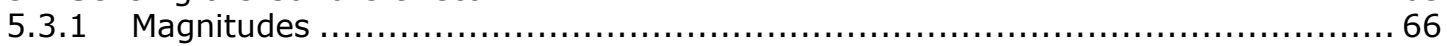

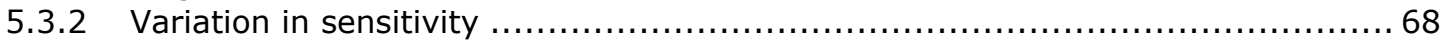

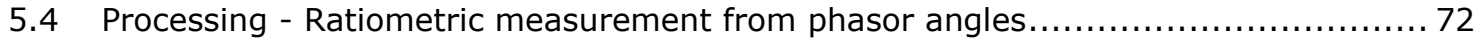

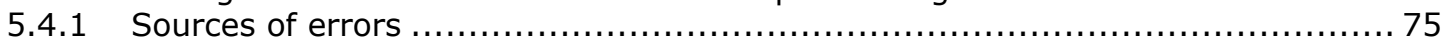

5.4 .2 Correction for rotation-pole shift using multiple sensors ............................ 78

5.4.3 Noise on ratiometric measurement due to noise on position sensor signals ......... 81

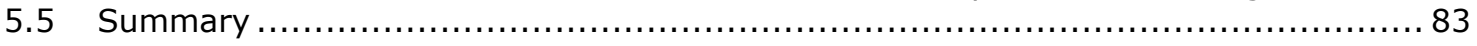

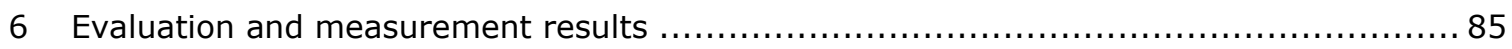

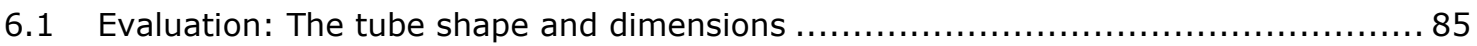

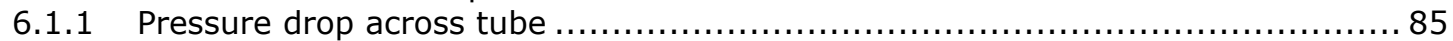

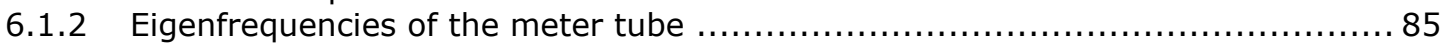

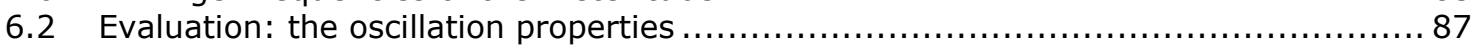

6.2.1 The $\mathrm{Q}$ factor and its relation to mass flow and the environment .................... 87

6.2.2 Actuation - power requirement and relation to the Q-factor ........................ 88

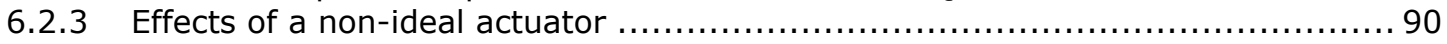

6.2.4 Temperature and tube eigenfrequencies ........................................... 91

6.2.5 Medium density and the tube eigenfrequencies.................................... 92

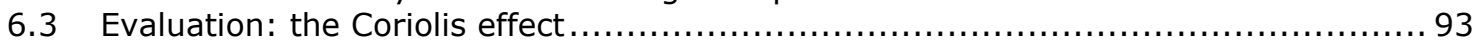

6.3.1 Density-related variation in the instrument sensitivity ............................ 93

6.3.2 Temperature-related variation in the instrument sensitivity ....................... 95

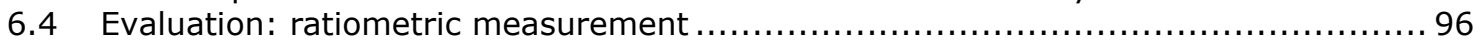

6.4.1 Inherent phase delays and mismatch in the instrument-construction .............. 96

6.4 .2 Pole shift estimation algorithm ...................................................... 96

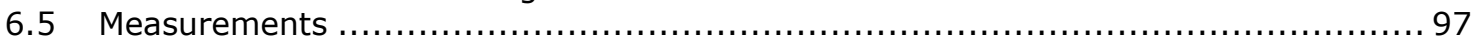

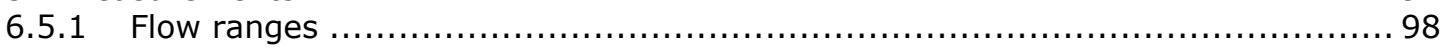

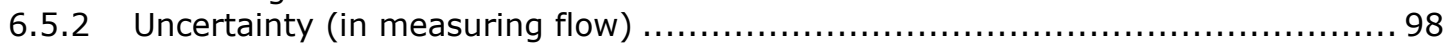

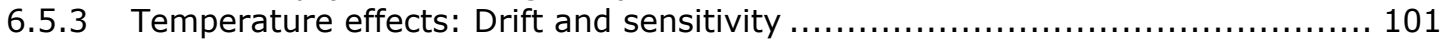

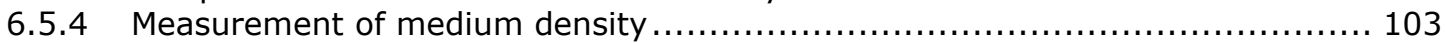

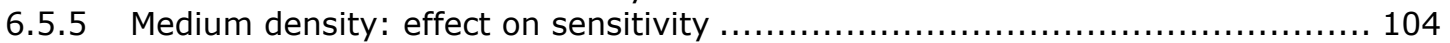

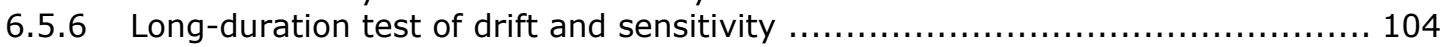

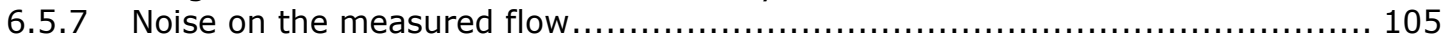

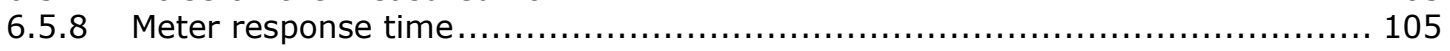




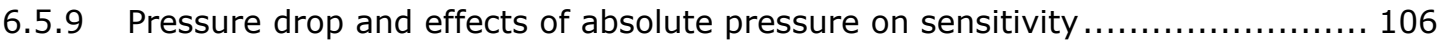

6.5.10 Influence of instrument attitude on offset and sensitivity ...................... 107

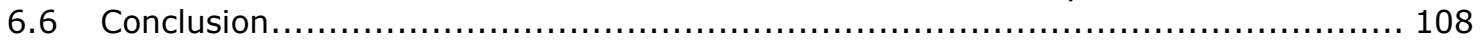

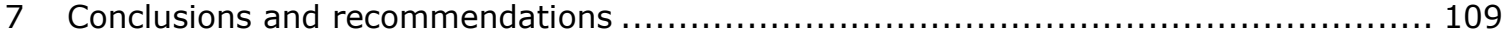

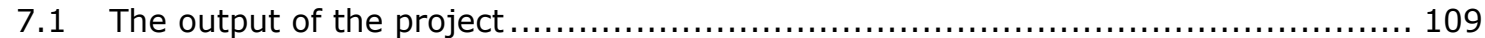

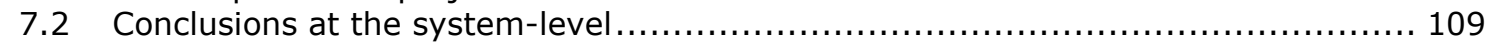

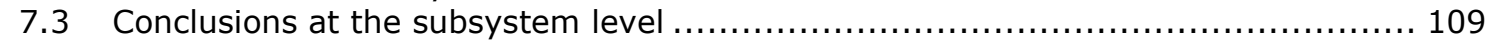

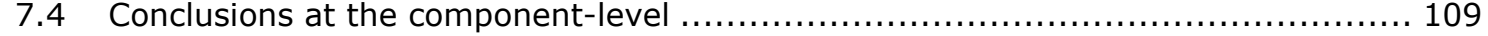

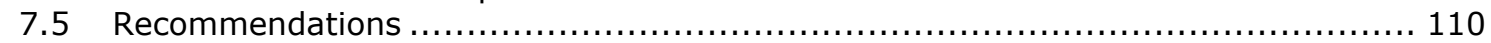

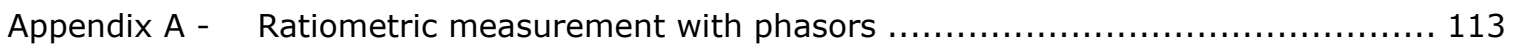

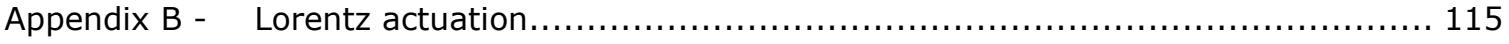

B.1 Dimensioning of the stator yoke of the Lorentz actuator .......................... 115

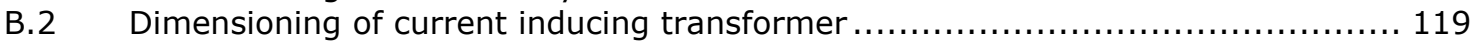

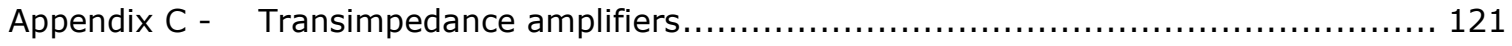

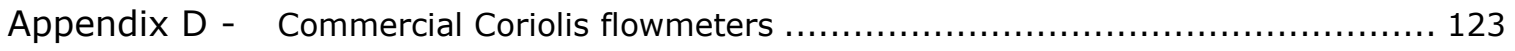

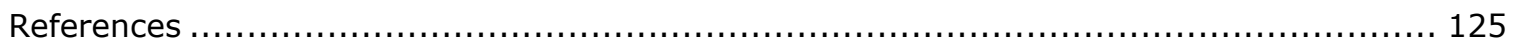

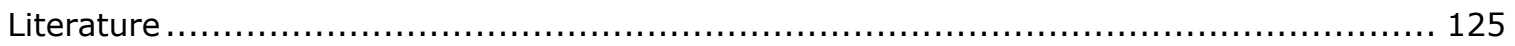

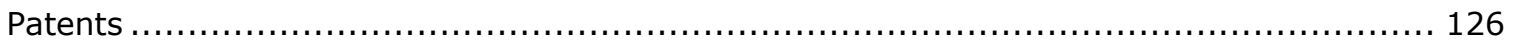

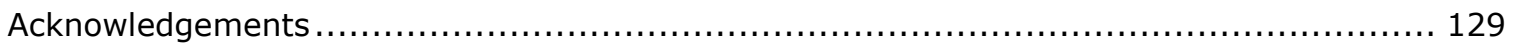

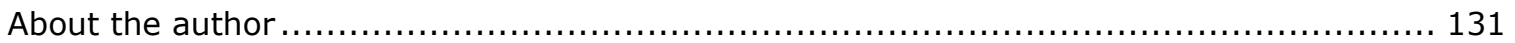





\title{
1 Introduction
}

\subsection{Mass flow rate measurement:}

Mass flow rate measurement, as the name suggests, is the measurement of the rate at which a quantity of a fluid, (expressed in terms of mass) crosses an imaginary boundary - for example the outlet of a tank. Before discussion of mass flow rate measurement, let us first consider measurement, in general. Measurement is process of associating numbers with physical quantities; but often this association has prerequisites and pitfalls.

As the "Guide to the expression of uncertainty in measurement - 1995", Paragraph 3.4.8 puts it (for measurement uncertainty - though the same principles hold for measurement, in general):

\begin{abstract}
"Although this guide provides a framework for assessing (uncertainty), it cannot substitute for critical thinking, intellectual honesty, and professional skill. The (evaluation of uncertainty) is neither a routine task nor a purely mathematical one; it depends on detailed knowledge of the nature of the measurand and of the measurement. The quality and utility (of the uncertainty) quoted for the result of a measurement therefore ultimately depend on the understanding, critical analysis, and integrity of those who contribute to the assignment of its value."
\end{abstract}

For the sake of critical analysis, in order to get a clear understanding of the nature of the measurand before delving into the measurement of mass flow rate, the individual elements are first discussed:

\subsubsection{The concept of mass}

Mass is a fundamental concept in physics, roughly corresponding to the intuitive idea of "how much matter there is in an object". Mass can be generally quantitatively expressed on the basis of two physical (observable) phenomena (see [1]): ${ }^{1}$

\section{Inertia:}

Inertial mass is a measure of an object's resistance to changing its state of motion when a force is applied. An object with small inertial mass changes its motion more readily, and an object with large inertial mass does so less readily.

\section{Gravitation:}

Every mass exerts a gravitational force of attraction on every other mass. The force of attraction between any one pair of masses is proportional to each of the masses in the pair and the inverse of the square of the distance separating the two. Under similar conditions, an object with a larger mass will exert a proportionately larger force of mutual attraction.

The inertial definition of mass is useful for predicting the behavior of tuning forks, billiard balls and deep-space rocket propulsion, while the gravitational definition of mass is useful in the context of bathroom weigh-scales and orbits of planets.

In the context of pendulums and free-fall, both definitions are simultaneously relevant - the comparison (and equivalence) of these definitions has been the subject of numerous experiments since Galileo. As of 2008, no proof of non-equivalence has been found (see [1]). ${ }^{2}$

The kilogram is the unit of mass, and is one of seven base units defined by the SI system. It is, to date, based upon a prototype (the international prototype kilogram "IPK") - a cylinder made of a platinum-iridium alloy and stored in a vault in the International Bureau of Weights and Measures in Sevres, France. Prior to this cylinder, the definition of a kilogram was based upon a liter of pure water at either the triple point $\left(0^{\prime} \mathrm{C}\right)$ or the maximum-density point $\left(4^{\prime} \mathrm{C}\right)$

${ }^{1}$ See also: http://en.wikipedia.org/wiki/Mass

${ }^{2}$ See also: http://en.wikipedia.org/wiki/Mass (Equivalence of inertial and gravitational masses) 


\subsubsection{The measurement of mass in a laboratory}

Though by no means the most accurate manner, it is convenient to measure mass in a laboratory indirectly in terms of weight. Assuming that the Earth's gravitational acceleration is constant and known in the laboratory, the mass of an object is proportional to its weight - the downward 'pull' force exerted by the Earth upon the object. For the purpose of this thesis, for liquids, weight measurements made by means of laboratory weigh-scales are treated as the reference ${ }^{3}$. The veracity of the meter may be checked by placing a reference calibration-mass upon the weigh-scales: this way, possible errors in the weigh-scales due to sensor gain, the definition of "down", and the local variation in earth's gravitational acceleration (together typically contributing to around $+/-0.2 \%$ error) can be eliminated.

Buoyancy effects due to local atmospheric pressure must be critically included in such measurements.

With laboratory-grade equipment and proper procedure, it is not challenging to estimate the mass of a liquid - from its weight - with a relative error smaller than $0.01 \%$.

For gases, a "piston prover" instrument is used as a reference - such an instrument uses the measurements of volume, temperature, pressure and information about the nature of a gas to indirectly state its mass.
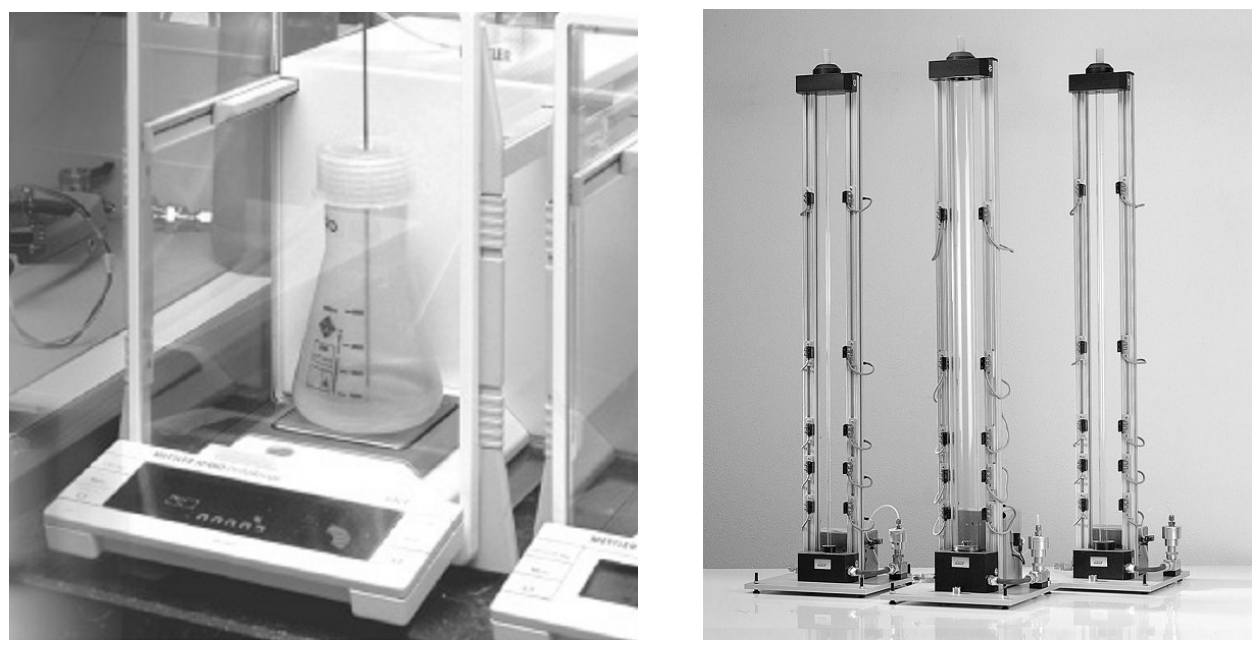

Figure 1.1 Weigh-scales and piston-prover instruments used for calibrating flowmeters

\subsubsection{The concept of flow}

The concept of flow, applicable to a material or fictitious extrinsic substance (water, heat, electric charge, road-traffic), is a measure conveying how much of the substance crosses a specified (imaginary) boundary. Examples:

- Twenty cubic meters of water flowed out of the tank

- One coulomb of charge flowed out of the battery terminal

- A lot of traffic (crossed the international boundary) today...

Flow rate is then the rate at which the substance crosses the boundary. Inherent to the definition of rate is the definition of time, which is discussed in the next section.

Examples:

- Water flowed out of the tank at the rate of twenty liters per second for 1000 seconds

- An Ampere of current flowed out of the battery terminal

(Implied: 1 Ampere corresponds to the rate 1 Coulomb per second)

- Traffic flowed into the Netherlands at the rate of 3000 vehicles per hour.

\footnotetext{
${ }^{3}$ E.g. Mettler-Toledo XP4002S weigh-scales: $4.1 \mathrm{~kg}$ full-scale and $0.01 \mathrm{~g}$ resolution
} 
Usually, a conduit is associated with a flow. This can be, for example, a tube, a conductor, or a road. While defining flow, it is often (unrealistically) assumed that the flow rate into a conduit (i.e. at its imaginary "inlet" boundary) is equal to the flow rate out of the conduit (i.e. at its "outlet" boundary). This may not be the case due to changing circumstances and capacity of the conduit, and should be critically considered while defining/interpreting flow and flow-rate.

\subsubsection{The concept of time}

The concept of time (see [2]) is too fundamental and philosophical in nature to discuss here. Practically, time is the representation of duration between two events. Time, like mass, has a unit (the second) that is one of the seven base units defined by the SI. Since 1967, the International System of Measurements bases the second on the properties of cesium atoms. SI defines the second (see [4]) as 9,192,631,770 cycles of the radiation that corresponds to the transition between two electron spin-energy levels of the ground state of the ${ }^{133} \mathbf{C s}$ atom. The term "rate" is used to express how much of something happens per unit time. As the passage of time is continuous ${ }^{4}$ (i.e. not discrete or granular, but smooth) for all practical purposes, a "constant rate" stays constant despite the small-ness of the time interval chosen to determine the rate.

\subsubsection{The measurement of time in a laboratory}

Time can be conveniently measured in a laboratory or within instruments by counting the oscillations of a crystal (usually quartz) resonator. The relative error with which large-scale time (a day or a year) can be measured in this manner is better than 10 parts per million. On a smaller time-scale, the error in the measurement of time (cycle-to-cycle jitter brought about due to thermal and other effects) has a standard deviation less than 10 picoseconds.

In a laboratory, mass flow rate measurement (measurement of the mass crossing a boundary per unit time i.e. $d \mathrm{~m} / d t$ ) is conveniently approximated by dividing accumulated mass by the elapsed time (i.e. $\Delta \mathrm{m} / \Delta t$ ), in a small time interval. As the measurement of time over practical time intervals (e.g. $1 \mathrm{~s}$ ) has a relative error that is orders of magnitude smaller than that of the measurement of mass, the error of the time-base of the reference instrument can be neglected.

\subsection{The need to measure the mass flow rate:}

As accepted by the SI, in its definition of the mole, the "amount of substance" can be compared in terms of its mass. That's to say - since the proposition of Avogadro's number, the following statement has not been disproved:

Equal "quantities" of the same substance (i.e. the same number of identical molecules) have the same mass.

Practically this means - barring the case of unknown isotopes - that for chemical reactions of any kind, it is advantageous to know the mass of reagents. In a batch-process, this (mixing reagents in a ratio of masses) can be done using weigh-scales. In a continuous process, massflow rate meters become essential ${ }^{5}$.

Volume flow (rate) measurement is often an acceptable description of the quantity or flow-rate of a substance. Water in a utilities meter and petrol and diesel fuel at a pumping station are de-facto expressed as a volume flow or a volume flow rate. Volume flow-rate measurement is generally simpler (and thus cheaper) than mass flow rate measurement. However typical causes of error when expressing the quantity of substance, when volume is measured instead of mass, are:

\footnotetext{
${ }^{4}$ Down to Planck time ( $\left.\sim 5.4 \mathrm{e}-44 \mathrm{~s}\right)$, where physical theories are believed to fail

${ }^{5}$ In modern times, mass flow rate controllers (meter + control-valve) are also used for batch operations (like bottle-filling or reagent dosing) due to their ease of automation, speed of operation, and inherent safety (no spillage or contamination during handling)
} 
- Density change

Cooler fluids are generally denser - i.e. have more substance per unit volume. Also, while dealing with compressible fluids (gases) the density is dependent on pressure. Phase change

Reagents such as ethylene or carbon dioxide may change phase (gas/liquid) during handling due to supercritical nature under pressure.

\subsection{The need to measure the small Mass flows:}

The measurement and control of small fluid flow-rates ('small' in this context is between 1 and $1000 \mathrm{~g} / \mathrm{h}$ ) is required in several applications in continuous reactors and batch operations in

- Semiconductor processing plants (dopants - silane, arsine, phosphine)

- Pilot-plants for petrochemical industries (ethylene)

- Pharmaceutical and food-and-beverage industry

The meters for such applications typically measure corrosive (titanium tetrachloride, hydrogen chloride) and hazardous (ethylene, arsine, phosphine) fluids and contamination-sensitive pharmaceutical agents.

\subsection{Existing mass flow measurement technology:}

Direct (i.e. not based upon volume measurements) and continuous mass flow rate meters available as standard industrial instruments are based on just one of two technologies:

- Thermal mass flow meters:

In these meters, the heat capacity of a known fluid medium is used in conjunction with its flow rate to transport heat towards or away from a sensor or heater. As such, the meters have to be calibrated per fluid medium, and are not suitable for unknown media and also not suitable if the medium properties change considerably (e.g. due to temperature, pressure, physical state, etc.).

- Coriolis mass flow meters:

In these meters, the Coriolis force generated by a fluid flowing in a rotating conduit is used to estimate the mass-flow.

Thermal mass flow rate meters (e.g. the Bronkhorst EL-flow controller in Figure 1.2) are available for gas flows as small as $1 \mathrm{mg} / \mathrm{h}$. Coriolis mass flow meters, on the other hand, are just recently commercially available for flows lower than $1 \mathrm{~g} / \mathrm{h}$.

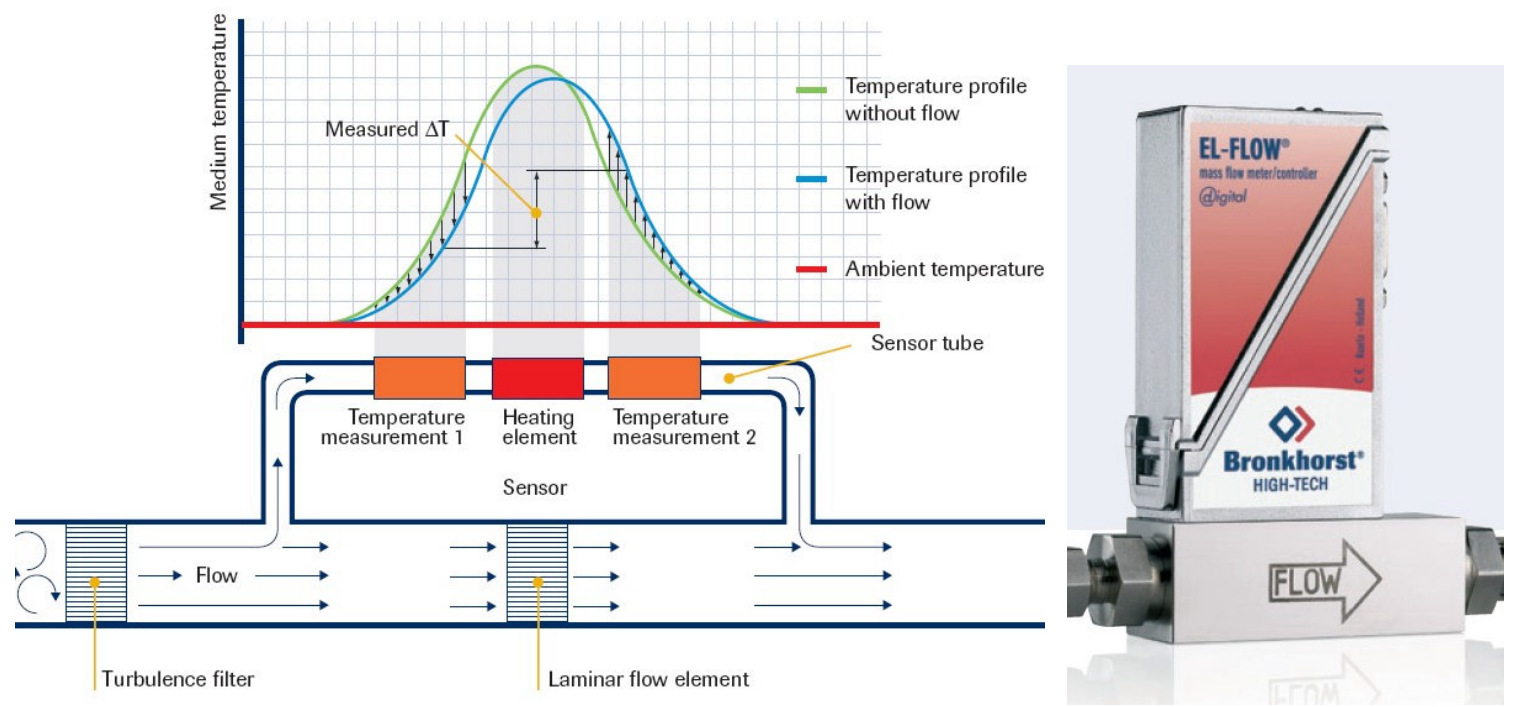

Figure 1.2 Thermal mass flow rate meter: Principle of operation (Bronkhorst EL-FLOW ${ }^{\circledR}$ ) 


\subsection{State of the art of Coriolis flow meters:}

At the time of this writing, there are about 10-15 manufacturers of flow-meters based on the Coriolis principle. An overview is presented in appendix $D$.

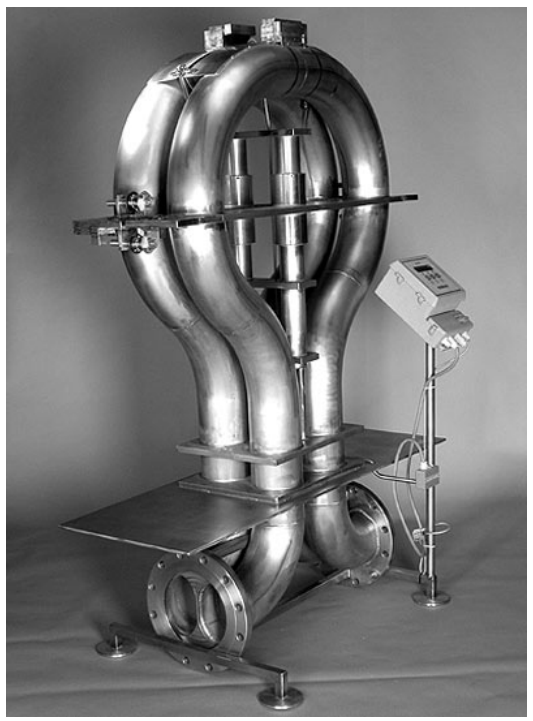

Figure 1.3 Large "omega" -shaped twin tube Coriolis meter for petrochemical applications (Rheonik RHM160)

Amongst the plethora of commercial Coriolis flowmeters, the ones intended for smaller flowranges are relevant for this research. For these instruments, the limiting factor usually is the zero drift. Four models are currently available with a specified "zero drift" lower than $0.3 \mathrm{~g} / \mathrm{h}$ :

- Emerson/Brooks Quantim

- Emerson/Micromotion LF2M

- Oval CNOOA

- Rheonik (Now GE) RHM015

\subsection{Motivation behind this research:}

Modern process industries, particularly IC fabrication and "pilot plants" for fine-chemical and pharmaceutical processes are moving towards higher process yields originating from finer process control, while staying flexible with smaller batch-sizes and a modular buildup of process instrumentation.

In the semiconductor industry, for example, the precursor CupraSelect ${ }^{\mathrm{TM}}$ is used to deposit conductive layers for IC interconnects. Other precursors make possible the so-called "low K dielectrics" and "high k dielectrics". Typical mass flow rates for these precursors are of the order of a few hundred grams per hour. The ability to accurately dose these is seen as an "enabling technology" facilitating advancement in IC miniaturization.

In the chemical/pharmaceutical industry, the new trend is scaling down the process to a "pilot plant", where results can be obtained rapidly, and relatively safely (due to the smaller scale) typically toxic and hazardous chemicals are used here. Here, temperature control of the whole process is also made easier, and smaller scales often mean less wastage. Here too, a flow rate of a few hundred grams per hour is required.

For the precise process control desired in both these industries, Coriolis meters are very suitable particularly as

- Measurement is independent of medium properties (accurate over phase-changes)

- As a side-effect, the instrumentation usage is flexible (one size fits all - no mediumcalibration is needed in the field) 
- Accuracy remains high over product lifetime - no clogging or wear

- $\quad$ Rapid meter response to flow-change

The mass-flow meters currently available are aimed at mass-flow of typically several hundred $\mathrm{kg} /$ hour and as such unsuitable for the smaller scales.

To this end, in collaboration with the University of Twente (formal theoretical background), Demcon (mechatronic design), and TNO (fluid dynamics), this research project has been undertaken.

\subsection{Challenges in the implementation:}

Coriolis meters scale poorly. That is to say, that generally speaking, their performance degrades as the overall size decreases. This scaling aspect is discussed further in Chapter 2. As with all flowmeters, it is desirable to make an instrument with high repeatability and small offset-drift. To avoid the need for characterization, linearity is also desirable. Due to the nature of measurement (discussed in the following chapters), unwanted forces of relatively large magnitudes interfere with the intermediate measurand, i.e. the Coriolis force, leading to large drift in the meter-offset. Designing a meter (for a small flow-rate) with an acceptably small drift is the most challenging task. The technical as well as the organizational aspects have been tackled with the 'Mechatronics-approach'6, for example, by means of a statically determined construction (see [3]), orthogonality of modes, constructional symmetries, strategic sensor and actuator placement and separation in frequency-domain. Processing (compensation for higher order physical effects) is also required in order to reduce sensitivity errors to less than $1 \%$. This is done by means of purely time-domain measurements, correction using multiple position sensors, and (sensitivity) correction for medium density and temperature.

\subsection{Organization of this thesis:}

The organization of this thesis is loosely based on the steps in a V-model of a systems development lifecycle [4], [8]. This is not merely a subjective choice. This project at the backbone of this thesis deals with the entire 'Mechatronic design cycle' (see [8]) of developing a state-of-the-art mechatronic measurement system. Rather than basing the chaptersegmentation on subsystems or on chronological order of research, the V-model approach is taken due to the nature of tasks involved from start to the finish.

\footnotetext{
${ }^{6}$ Mechatronics has come to mean the synergistic use of precision engineering, control theory, computer science, and sensor and actuator technology to design improved products and processes; insight into the mechatronics approach and also the Mechatronics design process is to be found in [8]
} 


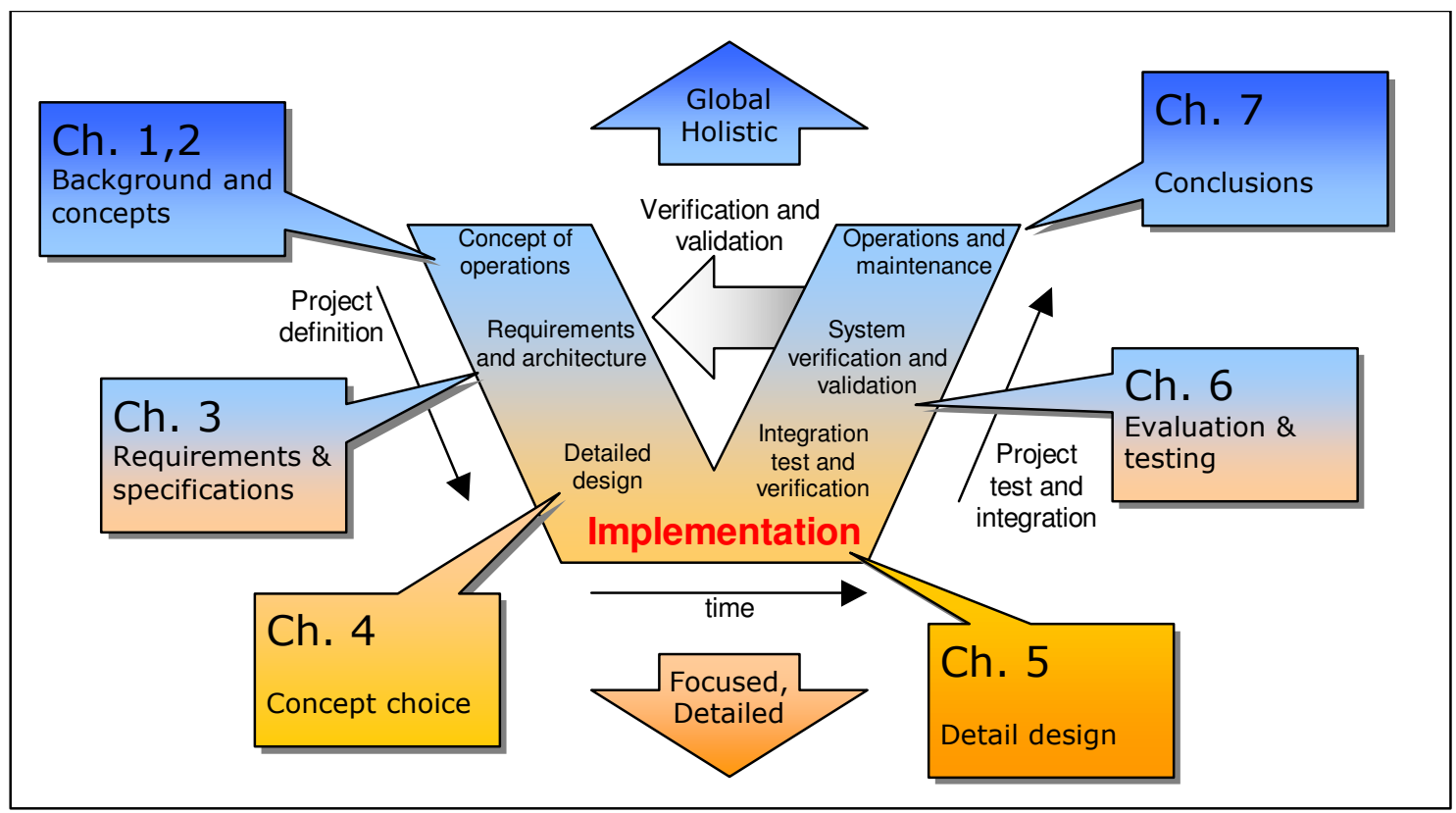

Figure 1.4 The ' $\mathrm{V}$ - model' of systems development lifecycle

Chapters 1 (this chapter) and 2 deal with the background of the research. In Chapter 3, the requirements $\&$ specifications laid out at the beginning of the project are stated and justified. Various concept choices are discussed in chapter 4 considering experience gained from intermediate proof-of-principle (PoP) experiments \& prototypes. In chapter 5 , the concepts of chapter 4 are worked out in detail; and their (theoretical) suitability to meet the requirements is demonstrated. In Chapter 6 , the evaluation (of concepts and intermediate prototypes) and testing of the definitive instrument is outlined.

As suggested by the 'feedback' arrow in the $\mathrm{V}$-model graphic, the development cycle is not a linear process; it has numerous iterative steps, to evaluate proposed concepts and to identify and iron-out unforeseen problems, amongst others. These iterations have been carried out through the project execution, but are not all listed in this thesis.

Finally, chapter 7 rounds up the thesis (and the development cycle), by giving conclusions about the project in terms of the impact of the chosen concepts, the outcome of the development cycle(s), and recommendations for future development. 



\section{Coriolis meters: Current performance and achieved improvements}

\section{Target setting:}

Coriolis meters are available as standard products for about 40 years; patents are to be found from as far back as the 1950s. Patents and literature refer for the most part to tube shapes and actuation, sensing and processing configurations. Literally hundreds of tube-shapes are to be found, many of them with immediately visible advantages and drawbacks. The one common factor in them all is, naturally, the generation and measurement of Coriolis force. In this chapter, we look at the basic operation of a Coriolis meter without implementation details, and form a generic model representative of the fundamental working. Subsequently, the current state of the art is considered (various existing Coriolis meters), their possible shortcomings are listed and the innovative elements of this work are presented against this background.

\subsection{Coriolis force}

Coriolis force, like centrifugal force is a pseudo-force (fictitious force) that appears to act on masses moving in a rotating reference-frame (see [1]). In a rotating coordinate frame, fictitious acceleration can be brought about due to the rotation of the reference frame.

The Coriolis force is named after Gaspard-Gustave de Coriolis, a French scientist who described it in 1835 in a paper titled "Sur les équations du mouvement relatif des systèmes de corps (On the equations of relative motion of a system of bodies)" This paper deals with the transfer of energy in rotating systems like waterwheels. Coriolis's name began to appear in meteorological literature (in the context of weather systems) at the end of the 19th century, although the term "Coriolis force" was not used until the beginning of the 20th century ${ }^{7}$. Today in popular science, the Coriolis effect is best known for explaining why weather systems in the the northern hemisphere spin counter-clockwise and in the southern hemisphere, clockwise.

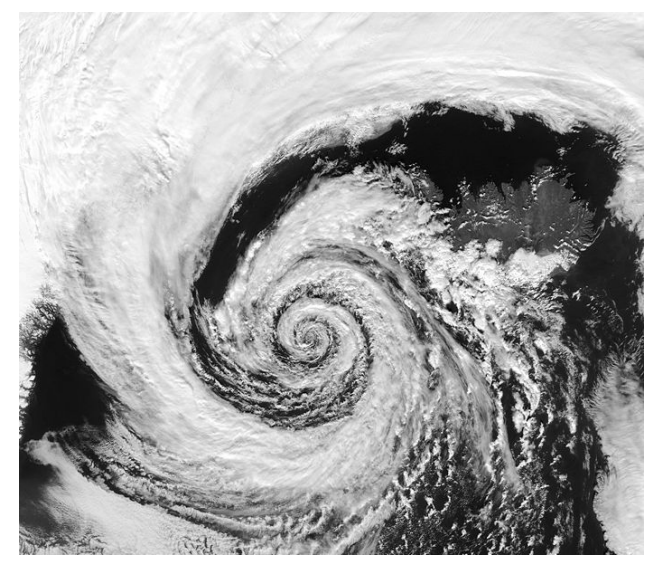

Figure 2.1 This low-pressure system over Iceland (the Northern hemisphere) spins counterclockwise due to balance between the Coriolis force and the pressure gradient force.

The relation between the time-derivative of any vector $\bar{B}$ in a coordinate frame ()$_{\text {rot }}$ rotating with an angular velocity $\overline{\dot{\theta}}$ and in an inertial coordinate frame ()$_{i n}$ can be expressed as:

\footnotetext{
${ }^{7}$ Additionally, animations illustrating the Coriolis effect \& Coriolis meters are to be found at http://en.wikipedia.org/wiki/Coriolis_effect and http://en.wikipedia.org/wiki/Mass_flow_meter
} 


$$
\left(\frac{d \bar{B}}{d t}\right)_{i n}=\left(\frac{d \overline{B_{i n}}}{d t}\right)_{r o t}+\bar{\omega} \times \bar{B}_{i n}
$$

If we express acceleration $\bar{a}$ as the time-derivative of velocity $\bar{v}$,

$$
\bar{a}_{i n}=\left(\frac{d \overline{v_{i n}}}{d t}\right)_{r o t}+\bar{\omega} \times \bar{v}_{i n}
$$

Similarly, if we express the velocity as the time-derivative of the position vector $\bar{r}$

$$
\overline{v_{i n}}=\overline{v_{r o t}}+\bar{\omega} \times \bar{r}
$$

(As there's only the rotation and no translation, $\bar{r}_{i n}=\bar{r}_{\text {rot }}$ )

The acceleration can now be expressed as

$$
\bar{a}_{i n}=\left(\frac{d\left(\overline{v_{r o t}}+\bar{\omega} \times \bar{r}\right)}{d t}\right)_{r o t}+\bar{\omega} \times\left(\overline{v_{r o t}}+\bar{\omega} \times \bar{r}\right)
$$

Or equivalently, as $\frac{d \overline{v_{r o t}}}{d t}=\overline{a_{r o t}}$ and $\frac{d \bar{r}}{d t}=\overline{v_{r o t}}$

$$
\bar{a}_{i n}=\bar{a}_{r o t}+\frac{d \bar{\omega}}{d t} \times \bar{r}+2 \bar{\omega} \times \overline{v_{r o t}}+\bar{\omega} \times(\bar{\omega} \times \bar{r})
$$

The "coordinate acceleration" in the rotating frame is thus

$$
\bar{a}_{\text {rot }}=\underbrace{\bar{a}_{\text {in }}}_{\text {physical acceleration }}-\underbrace{\underbrace{\frac{d \bar{\omega}}{d t} \times \bar{r}}_{\text {Euler acceleration }}-\underbrace{2 \bar{\omega} \times \overline{v_{\text {rot }}}}_{\text {Coriolis acceleration }}-\underbrace{\bar{\omega} \times(\bar{\omega} \times \bar{r})}_{\text {centrifugal acceleration }}}_{\text {fictitious; due to a rotating coordinate frame }}
$$

which is the physical acceleration, exerted by external forces on the object, plus additional terms associated with the geometry of the rotating reference frame.

For an object having mass $\mathrm{m}$ in this rotating reference-frame, the fictitious acceleration terms bring about:

\section{- Centrifugal force:}

$$
\bar{F}_{\text {centrifugal }}=m \cdot(-\bar{\omega} \times(\bar{\omega} \times \bar{r}))=\left(m \omega^{2} r\right) \hat{r}
$$

in the radial direction

\section{- Coriolis force:}

$$
\bar{F}_{\text {Coriolis }}=m\left(-2 \bar{\omega} \times \overline{v_{\text {rot }}}\right)
$$

in a direction perpendicular to the rotation and velocity 


\section{- Euler force:}

$$
\bar{F}_{\text {Euler }}=m\left(-\frac{d \bar{\omega}}{d t} \times \bar{r}\right)
$$

caused by the angular acceleration of the coordinate frame

Going a step further, if instead of a mass, we have a continuous flow (e.g. water in a rotating tube), the individual flowing elements (globs of water) can be thought of as masses together contributing to the fictitious forces.

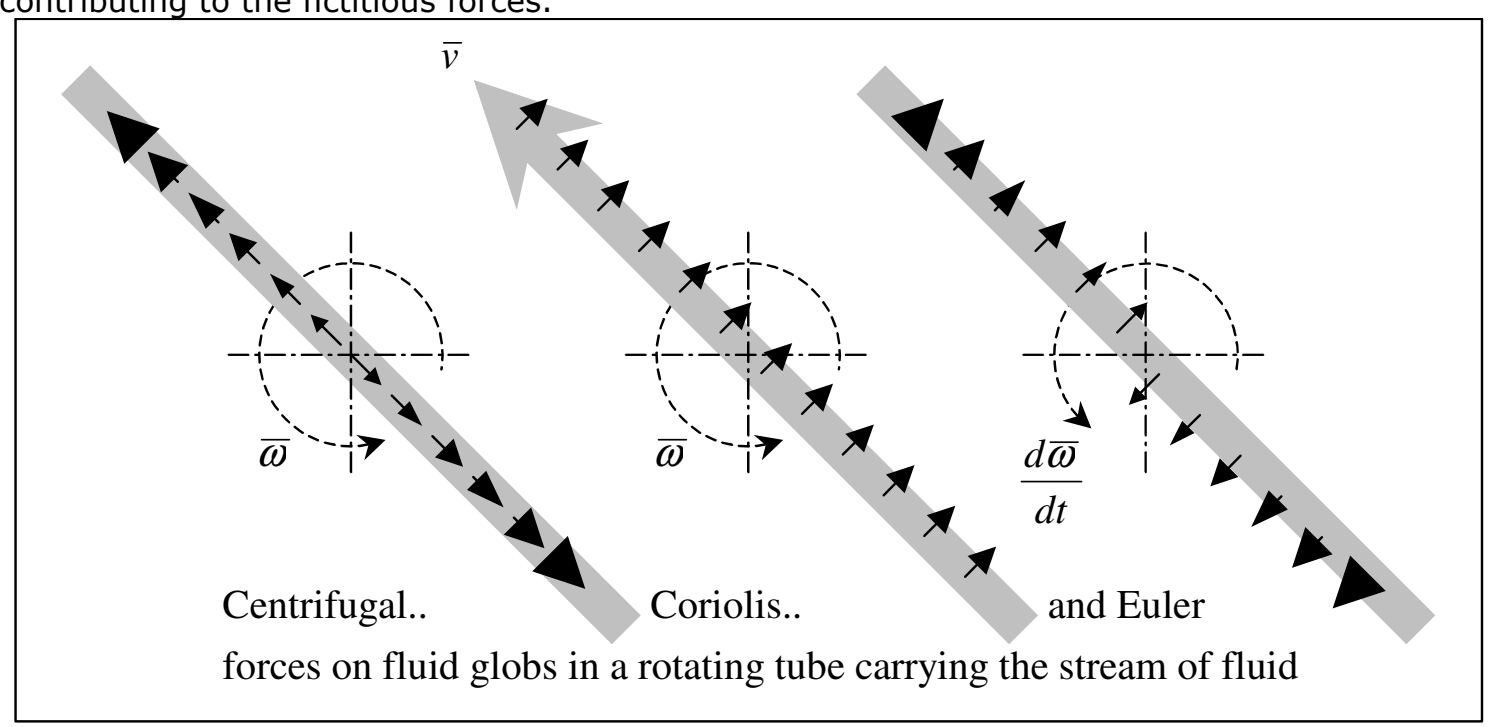

Figure 2.2 Pseudo-forces acting upon fluid in a rotating tube

For the particular case of the Coriolis force, where the velocity plays a role, consider a glob of fluid with mass $d m$, taken as a thin slice of the tube of total length $L$; the thickness of the slice is $d l$, the sectional area is $A$ and the density of the fluid is $\rho$. The tube rotates with an angular velocity $\bar{\omega}$

Summing over all globs:

$$
\left(\bar{F}_{\text {Coriolis }}\right)_{\text {total }}=\int_{l=0}^{L}-2 \bar{\omega} \times \bar{v}_{\text {rot }} \cdot \overbrace{\rho \cdot A \cdot d l}^{\text {aglob of fluid }}
$$

Notice, however, that $\overline{v_{\text {rot }}} \cdot A$ is the volume flow rate and $\overline{v_{\text {rot }}} \cdot \rho \cdot A$ is actually the mass-flowrate $\Phi_{m}$. We can rewrite the expression for Coriolis force as:

$$
\left(\bar{F}_{\text {Coriolis }}\right)_{\text {total }}=\int_{l=0}^{L}-2 \bar{\omega} \times \overline{\Phi_{m}} \cdot d l=-2 l \cdot\left(\bar{\omega} \times \overline{\Phi_{m}}\right)
$$

This relation forms the basis for all Coriolis mass flow rate meters ${ }^{8}$.

${ }^{8}$ For a more intuitive explanation about the generation of Coriolis force, see [9]. [6] gives thorough treatment of rotating reference frames and gyroscopes. 


\subsection{Harnessing the Coriolis force}

From equation 2.8, is seen that in order to generate Coriolis force, a conduit of length $L$ carrying mass-flow (with MFR $\Phi$ ) is needed. When this conduit is rotated with an angular rate of $\omega$ (henceforth referred to as $\dot{\theta}$ to avoid confusion with the symbol for eigenfrequency), it will result in a Coriolis force $F_{c o r}$ proportional to $\Phi$. The following illustrative construction has these components (mass-flow, length and angular rate) and is perhaps the simplest possible usable Coriolis MFR meter.

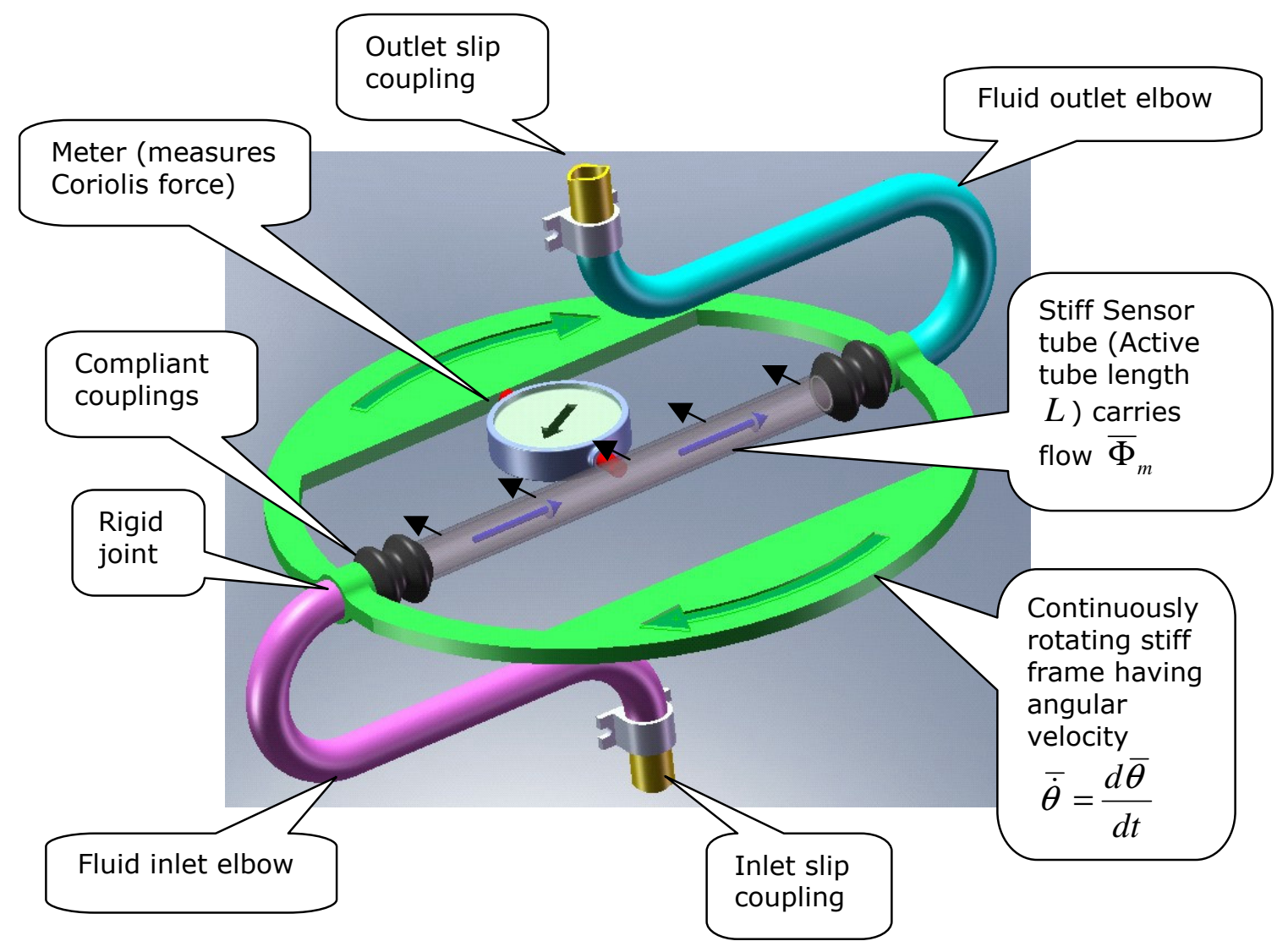

Figure 2.3 An illustrative rotating-tube Coriolis MFR meter

In this construction, fluidic mass-flow is introduced into a so called "active tube length" by means of two slip-couplings and (compliant) bellows. The inlet and outlet are fixed, while the tube construction in between is driven to rotate by means of an external engine, such as an electromotor. A stiff frame couples the feeding sections of pipe so that the inlet and outlet "elbows", together with the frame, form a stiff rotating construction. A (stiff) force sensor is positioned between the rigid frame and the central straight piece of 'sensor' tube between the two bellows (constituting the active tube-length).

The resulting construction is rigid (meaning that the Coriolis force does not distort the tube geometry). As the construction rotates, and a mass-flow is forced through it, all rotating parts of the tube (including the elbows) will experience a Coriolis force. This force will be restrained by the stiff construction - i.e. bearings around in the slip-couplings, and the rigid frame. The (sideways) Coriolis force in the middle section of the tube will also be restrained, but via the (stiff) force-sensor. The reading on this sensor will thus indicate the net Coriolis force acting on the central rotating tube section, pushing against the rigid frame. 
To get an idea about the magnitudes involved, let us consider for the construction in Figure 2.3 a tube of length $0.2 \mathrm{~m}$ and cross-section $1 \mathrm{sq} . \mathrm{cm}$ (irrelevant) rotating at 300 RPM (i.e.

$5 \mathrm{~Hz}$, or $31.4 \mathrm{rad} / \mathrm{sec}$ ). Consider water flowing through this tube at the rate of $0.1 \mathrm{~kg} / \mathrm{s}-$ about a liter in 10 seconds.

The Coriolis force this tube will generate is:

$$
\left|\bar{F}_{\text {Coriolis }}\right|=\left|-2 l \cdot\left(\overline{\dot{\theta}} \times \overline{\Phi_{m}}\right)\right|=2 \times 0.2 \times 31.4 \times 0.1 \cong 1.25[N]
$$

This is small compared to the centrifugal force due to water column in one half of the tube (about $5 \mathrm{~N}$ per half) and somewhat larger than the rate-of-change of momentum in each of the elbows (about $0.2 \mathrm{~N}$ per elbow)

A rotating meter construction, remarkably similar to this one, has been patented as far back as $1953^{9}$.

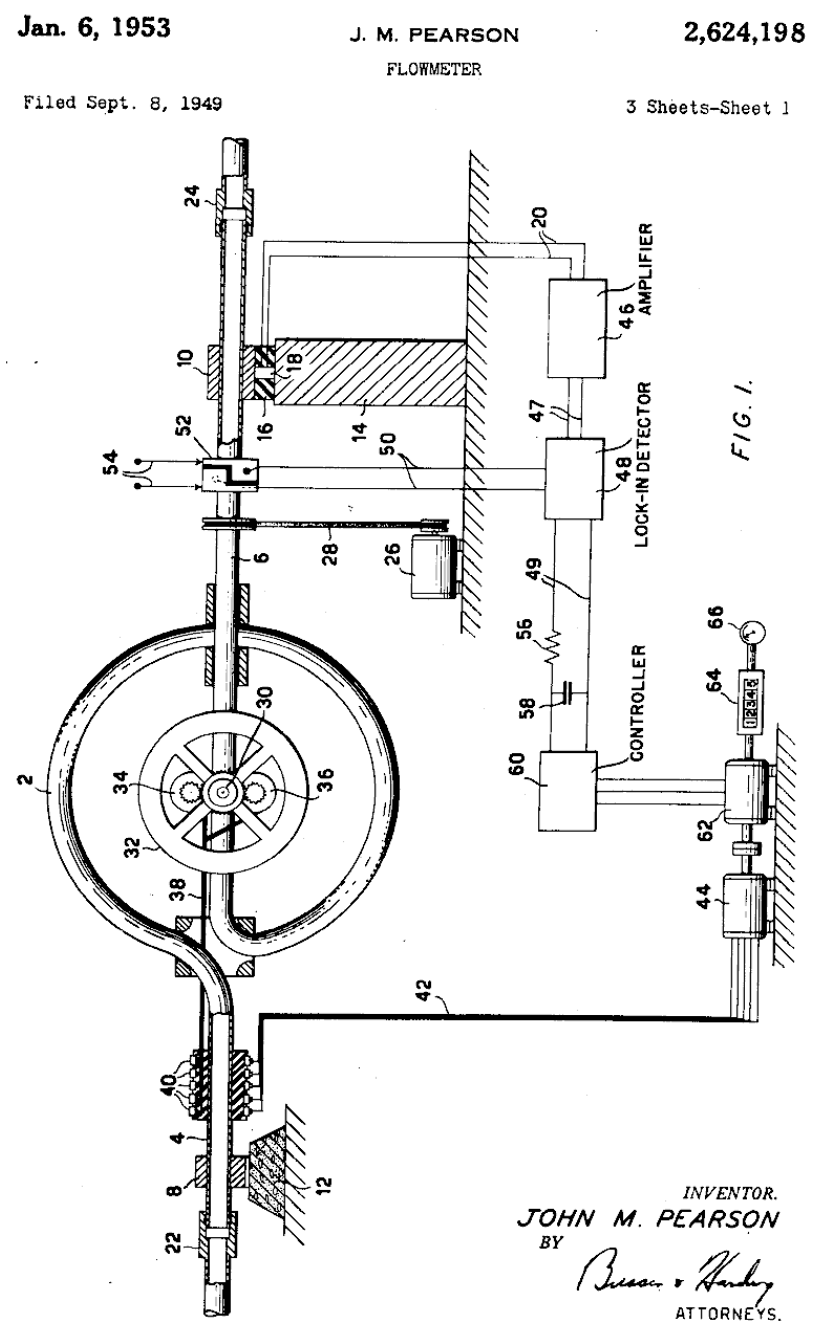

Figure 2.4 A rotating-tube Coriolis meter construction (US patent 2624198)

A severe drawback of the rotating construction (as also noted by this inventor) is the presence of slip-couplings. Wear, material incompatibility, and complexity of construction \& maintenance make such a construction impractical.

\footnotetext{
${ }^{9}$ US patent 2624198, Inventor: J. M. Pearson
} 
An approach taken by several inventors in the 50's is one where the "sensing element" in the tube undergoes oscillatory -rather than continuous- rotation. This "twist" motion, similar to the dance style invented around the same period, is still used in the present day. The basic idea is that a small angular deflection (though with high angular rates, as will be seen) is brought about by means of elastic deformation of the fluid-carrying conduit. This deflection is oscillatory in nature. As such, the need for slip-couplings is eliminated. Of course, this means that the generated Coriolis force too is oscillatory in nature. Possible ill effects of this (aliasing of fast-changing flow due to pulsating readout) can be avoided by choosing a sufficiently high oscillation frequency.

\subsection{The oscillatory rotating tube}

In order to generate a rotation in a tube section by elastic deformation, a form suitable to such deformation is needed. Consider, for the sake of illustration, a U-shaped tube. This is not by far the most ideal or even the simplest form - it is merely easy to visualize and to illustrate.

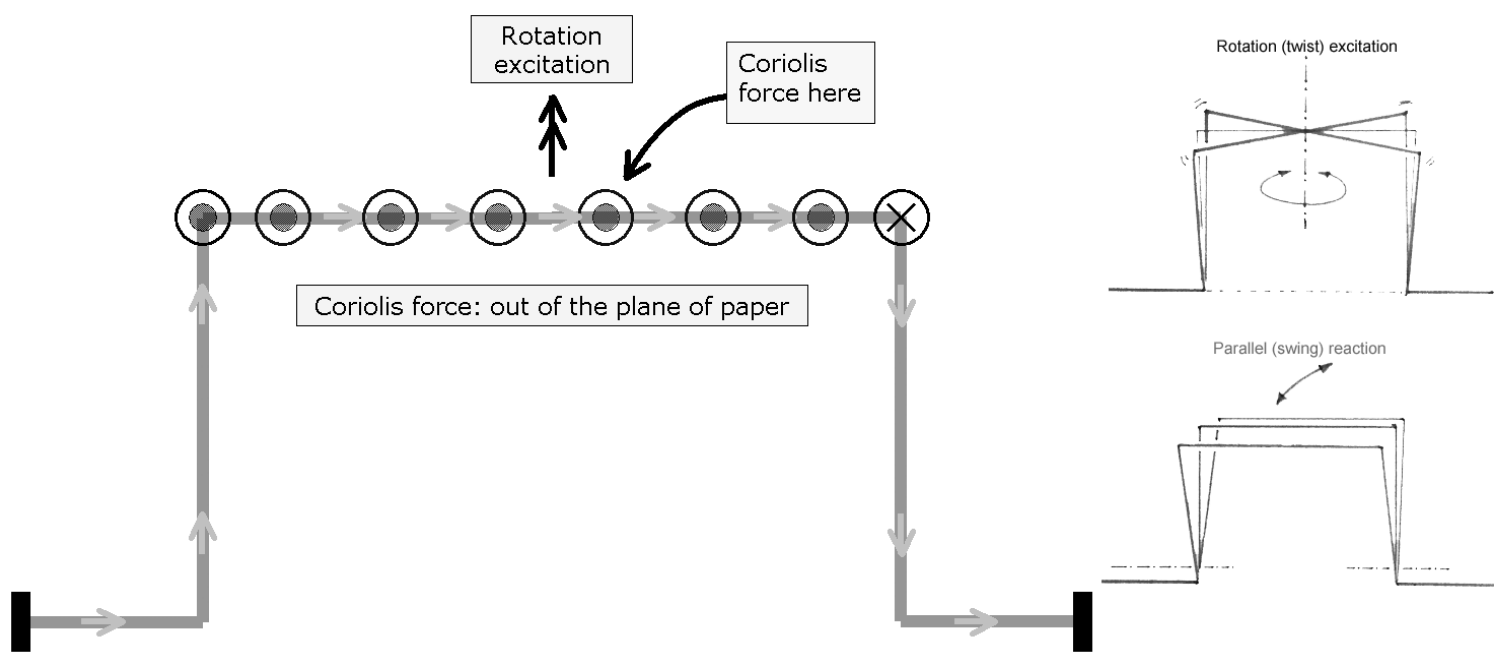

Figure 2.5 Coriolis force in an oscillatory-rotating U-tube driven in a "twist" motion

In this construction a rotation excitation is imposed upon the central portion of the tube, as shown. The two vertical sections can bend and create the necessary compliance to facilitate this rotation. For a particular cross section the "area moment of inertia" can be determined. E.g., for a cylindrical tube,

$$
I_{O}=\frac{\pi}{64}\left(O D^{4}-I D^{4}\right)\left[\mathrm{m}^{4}\right]
$$

For a given material, the elastic modulus $E$ can be looked up. For a bending element of height $h$, the tip-stiffness is then:

$$
k=\frac{3 \cdot E \cdot I_{O}}{h^{3}}[\mathrm{~N} / \mathrm{m}]
$$

The rotating tube of length $l$ sees torsion stiffness contributed by both the vertical elements:

$$
k_{\text {tor }}=2 \cdot k \cdot\left(\frac{l}{2}\right)^{2}=\frac{1}{2} k \cdot l^{2}[\mathrm{~N} \cdot \mathrm{m} / \mathrm{rad}]
$$

where $l / 2$ is the arm of each of the springs from the torsion axis. 
This is by no means an exact estimate of the stiffness. The torsion in the inlet and outlet tubes (which decreases the overall stiffness), the torsion in the rotating length that couples the left and right bending elements (which increases the overall stiffness), and other higher order effects have been neglected. It does give fair estimates for order-of-magnitude calculations.

As an example, we reproduce the circumstances in the example tube from Figure 2.5 here. It is assumed that the height $h$ of the bending elements is $0.1 \mathrm{~m}$.

\begin{tabular}{|l|c|c|c|l|}
\hline Parameter & Symbol & Value & Units & Comment \\
\hline Length of rotating element & $l$ & 0.2 & {$[\mathrm{~m}]$} & \\
\hline Internal tube-diameter & $I D$ & 0.010 & {$[\mathrm{~m}]$} & Approx. $1 \mathrm{~cm}^{2}$ C.S. area \\
\hline Outer tube-diameter & $O D$ & 0.014 & {$[\mathrm{~m}]$} & 2 mm wall thickness \\
\hline Area moment of inertia & $I_{O}$ & $1.4 \mathrm{e}-9$ & {$\left[\mathrm{~m}^{4}\right]$} & \\
\hline Height of bending elements & $h$ & 0.1 & {$[\mathrm{~m}]$} & \\
\hline Tube material & $E$ & $2 \mathrm{e} 11$ & {$\left[\mathrm{~N} / \mathrm{m}^{2}\right]$} & {$[\mathrm{Pa}]$} \\
\hline Elasticity modulus of material & $k$ & $8.37 \mathrm{e} 5$ & {$[\mathrm{~N} / \mathrm{m}]$} & \\
\hline $\begin{array}{l}\text { Bending stiffness of each of } \\
\text { the vertical elements }\end{array}$ & $k_{\text {tor }}$ & $1.67 \mathrm{e} 4$ & {$[\mathrm{Nm} / \mathrm{rad}]$} & \\
\hline $\begin{array}{l}\text { Torsion stiffness experienced } \\
\text { by tube }\end{array}$ & $\dot{\theta}$ & 31.4 & {$[\mathrm{rad} / \mathrm{s}]$} & Similar to the tube in 2.2 \\
\hline Desired angular rate & $f_{o s c}$ & 50 & {$[\mathrm{~Hz}]$} & $\begin{array}{l}\text { Arbitrary - high enough to } \\
\text { follow flow pulsations up } \\
\text { to } 10 \text { Hz }\end{array}$ \\
\hline $\begin{array}{l}\text { Oscillation frequency } \\
\text { Required angular amplitude }\end{array}$ & $\theta_{t w}$ & 0.1 & {$[\mathrm{rad}]$} & $\begin{array}{l}\text { To achieve the desired } \\
\text { angular rate }\end{array}$ \\
\hline
\end{tabular}

Momentarily neglecting possible plastic deformation in the tube, let us calculate the force exerted by each of the bending elements:

\begin{tabular}{|l|c|c|c|l|}
\hline Peak deflection at corner & $\tilde{d}$ & 0.01 & {$[\mathrm{~m}]$} & $0.1 \mathrm{~m} \cdot 0.1 \mathrm{rad}$ \\
\hline $\begin{array}{l}\text { Peak spring-force in bending } \\
\text { element }\end{array}$ & $F_{b}$ & $8.37 \mathrm{e} 3$ & {$[\mathrm{~N}]$} & $\begin{array}{l}6700 \text { times larger than } \\
\text { the peak Coriolis force!! }\end{array}$ \\
\hline
\end{tabular}

Furthermore oscillation requires that each tube-element accelerates and decelerates to make possible the twist motion. This also causes inertial forces:

\begin{tabular}{|l|c|c|c|l|}
\hline Peak deflection at corner & $\tilde{d}$ & 0.01 & {$[\mathrm{~m}]$} & $0.1 \mathrm{~m} \cdot 0.1 \mathrm{rad}$ \\
\hline Oscillation frequency & $f_{\text {osc }}$ & 50 & {$[\mathrm{~Hz}]$} & \\
\hline Peak acceleration at corner & $\frac{d^{2} \tilde{d}}{d t^{2}}$ & 987 & {$\left[\mathrm{~m} / \mathrm{s}^{2}\right]$} & $=\tilde{d}(2 \pi \cdot f)^{2}$ \\
\hline $\begin{array}{l}\text { Lumped inertia (tube plus } \\
\text { medium) expected at a corner }\end{array}$ & $\begin{array}{c}m \\
\text { (approx) }\end{array}$ & 0.068 & {$[\mathrm{~kg}]$} & $\begin{array}{l}\text { Rotating pieces: half tube } \\
\text { and bending element }\end{array}$ \\
\hline $\begin{array}{l}\text { Peak inertial-force at each } \\
\text { tube-corner }\end{array}$ & $F_{i}$ & 67 & {$[\mathrm{~N}]$} & $\begin{array}{l}\text { About 50x larger than the } \\
\text { peak Coriolis force }\end{array}$ \\
\hline
\end{tabular}

To summarize, in an oscillating-tube construction, we end up with elastic and inertial forces that are several orders larger than the peak Coriolis force. If a flow that is a factor 1000 smaller than the peak flow is to be reliably detected, a system that can separate the Coriolis force from other forces present in the tube with a 1-in-6700000 ratio is required.

For example, if the Coriolis force is to be inferred directly with strain gages measuring the strain in the left and right benders, these two strain-gages have to discriminate the small common-mode strain caused by the Coriolis force from the large difference-mode strain caused by excitation motion. As such, they have to be matched to better than the said 1 part 
in 6.7 million $^{10}$. This requirement can be avoided by measuring motion instead of strain - near the rotation axis, the excitation motion is very small, and thus the Coriolis (swing) motion is relatively larger.

Furthermore, to create the oscillatory rotation motion, an actuator that can deliver a peak torque $\mathrm{T}$ of $1.67 \mathrm{e} 3 \mathrm{~N} \cdot \mathrm{m}$ (peak power: $26 \mathrm{~kW}\left(=\frac{1}{2} \mathrm{~T}_{\text {peak }} \cdot \dot{\theta}_{\text {peak }}\right){ }^{11}$ ) Such a brute-force approach can be avoided, for example, by using resonance. Such systems and constructions are presented in chapter 4.

Of course it is possible to change some tube-parameters to reduce the 1-in-6.7e6 disparity and the large excitation forces:

\begin{tabular}{|c|c|c|}
\hline Parameter changed & The improvement & The limitation \\
\hline Reduce wall-thickness & $\begin{array}{l}\text { Mass decreases } \\
\text { Stiffness decreases }\end{array}$ & $\begin{array}{l}\text { Tube must withstand medium } \\
\text { pressure without bursting }\end{array}$ \\
\hline $\begin{array}{l}\text { Reduce tube diameter } \\
D\end{array}$ & $\begin{array}{l}\text { Mass decreases as } D^{2} \text { while } \\
\text { Stiffness decreases as } D^{4}\end{array}$ & $\begin{array}{l}\text { Pressure-drop due to flow } \\
\text { increases }\end{array}$ \\
\hline $\begin{array}{l}\text { Increase tube-length } \\
\text { (more Coriolis force) }\end{array}$ & $\begin{array}{l}\text { Stiffness decreases but mass } \\
\text { increases }\end{array}$ & Pressure-drop increases \\
\hline $\begin{array}{l}\text { Increase bender- } \\
\text { height } h\end{array}$ & $\begin{array}{l}\text { Stiffness decreases as } h^{3} \text { but mass } \\
\text { increases }\end{array}$ & Pressure-drop increases \\
\hline $\begin{array}{l}\text { Increase twist } \\
\text { amplitude }\end{array}$ & $\begin{array}{l}\text { Coriolis force increases, but so does } \\
\text { the deflection effort and inertial force }\end{array}$ & $\begin{array}{l}\text { Plastic deformation of tube } \\
\text { material }\end{array}$ \\
\hline $\begin{array}{l}\text { Increase the } \\
\text { oscillation-frequency }\end{array}$ & $\begin{array}{l}\text { Coriolis force increases, as does the } \\
\text { inertial force }\end{array}$ & $\begin{array}{l}\text { Sideways pressure-waves in } \\
\text { flow-channel }\end{array}$ \\
\hline $\begin{array}{l}\text { Decrease the } \\
\text { oscillation-frequency }\end{array}$ & $\begin{array}{l}\text { Lower inertial forces at the cost of } \\
\text { smaller Coriolis force }\end{array}$ & $\begin{array}{l}\text { Aliasing of flow-pulses } \\
\text { (causing large measurement } \\
\text { error) }\end{array}$ \\
\hline
\end{tabular}

\subsection{A different take on the oscillatory rotating tube}

We chose to impose oscillating motion upon the U-tube in section 2.3 in the form of a twist. In this situation, the horizontal (top section) of the " $U$ " rotates, primarily giving rise to Coriolis forces. A different manner of excitation of the same U-tube is also conceivable:

\footnotetext{
${ }^{10}$ Typical load-cells offer gain matching to the order of 1 -in-1000. A stable (over time) match of 1 in 6700000 is impractical.

${ }^{11}$ Actuation on stiffness-line: $\mathrm{T}_{\text {peak }}$ and $\dot{\theta}_{\text {peak }}$ are $90^{\circ}$ out-of-phase
} 


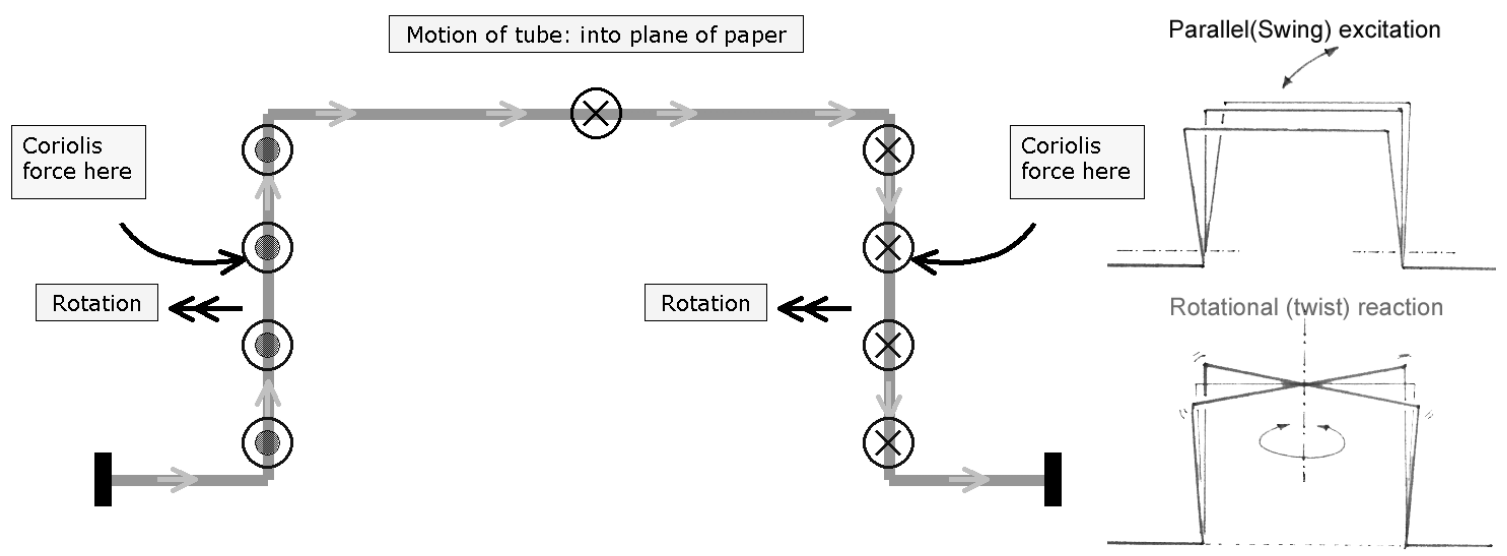

Figure 2.6 Coriolis force(s) in a U-tube driven in a "swing" motion

In this case, the vertical limbs rotate, and in the two limbs arises Coriolis force, as an antiparallel pair (a couple). This couple deflects the tube in a twist motion. While this second concept is too perfectly feasible, it has two major disadvantages:

- The point on the tube where the reaction (twist motion) can be measured is the top section. However, a large excitation (swing) motion is always present here; If motion sensing is to be used, the sensor chain gets the additional task of tolerating and removing the large excitation signal.

- The tube construction is not symmetric about the rotation-axis. Symmetric actuation is difficult, and a part of the actuation effort can affect the reaction mode motion, and get misinterpreted as Coriolis force.

- The stiffness added by the attachment points lets the Coriolis forces make smaller deflection on the rotation (reaction) axis.

\subsection{The challenge in scaling down}

Suppose that a particular tube shape is chosen for the purpose of building a Coriolis massflowmeter. Suppose also, for simplicity, that the tube used for this shape is uniform - it does not change in cross section and thickness over the active length. The thinner the tube wall, the less mass and stiffness it will provide to the Coriolis force, resulting in a larger Coriolis motion (advantageous). However, the tube has to withstand a certain fluid pressure - this value for pressure (together with the strength of the tube material) fixes the minimum ratio of the wall thickness to the inner diameter of the tube. Consequently (as the wall thickness can be varied in a fixed ratio with the $I D$ ) it is possible to "scale" the tube (geometry and also cross-section) with a scaling factor " $S F$ ".

For a useful flowmeter, let us define the nominal maximum flow rate as the flow rate that causes a particular pressure drop across the tube. Making the tube thinner is impractical. For comparison, consider also that the angular excitation amplitude of the construction at any scale is constant (related to material fatigue). Consider now what happens with the same tube at a different scale:

Nominal maximum flow-rate (laminar) ${ }^{12}: \frac{\Delta P \cdot \pi \cdot I D^{4} \cdot \rho}{128 \cdot \eta \cdot L} \Rightarrow$ varies as $S F^{3}$

Corner stiffness (equivalent linear stiffness): $\frac{O D^{4}}{h^{3}} \Rightarrow \frac{S F^{4}}{S F^{3}} \Rightarrow$ varies as $S F$

\footnotetext{
12 The expressions for these quantities have been merely listed here, they are explained in Ch. 5.1.1
} 
Excitation - linear excursion (of corner): varies as $S F$

Excitation force (=stiffness $\times$ excitation): varies as $S F^{2}$

Tube inertia: varies as $S F^{3}$ (related to the volume)

Tube eigenfrequency: $\omega=\sqrt{\frac{k}{m}} \Rightarrow \sqrt{\frac{S F}{S F^{3}}} \Rightarrow$ varies as $\frac{1}{S F}$

Active tube length: $L \Rightarrow$ varies as $S F$

Flow-rate per excitation-force: $F p E \Rightarrow \frac{S F^{3}}{S F^{2}} \Rightarrow$ varies as $S F$

Coriolis force per excitation force: $\quad \frac{\overbrace{\frac{2 \cdot(\text { massflowrate }) \cdot L \cdot \dot{\theta}}{\text { exc }}}^{\text {Coxcitation force })}}{\Rightarrow 2 \cdot(F p E) \cdot L \cdot \overbrace{\theta_{\text {exc }} \cdot \omega_{\text {eig }}}^{\text {peak angular vel }} \Rightarrow 2 \cdot(S F) \cdot S F \cdot 1 \cdot \frac{1}{S F} \Rightarrow \text { varies as } S F}$
$\Rightarrow$

Effectively, as the tube is scaled-down, the relative magnitude of the Coriolis force reduces, making it more challenging to measure the Coriolis force in presence of the other (relatively much larger) forces arising from tube excitation. 


\subsection{Innovation areas, compared with the state of the art}

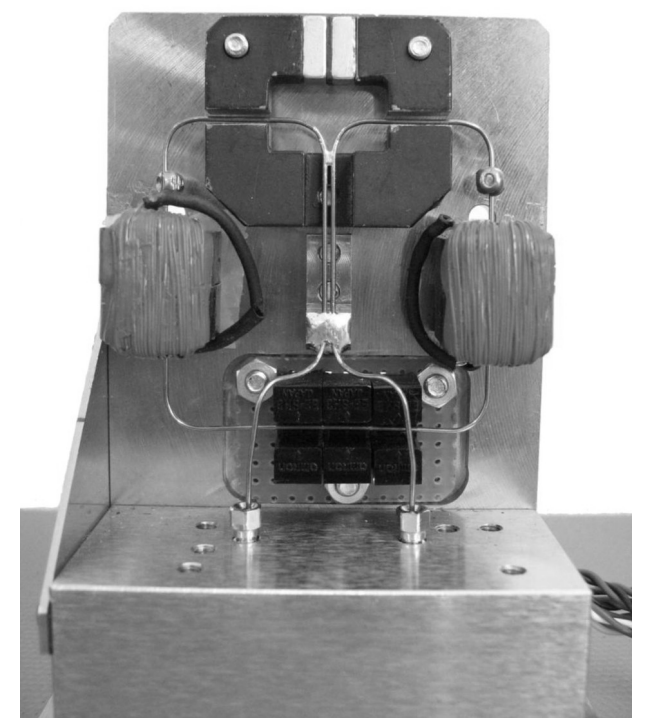

Figure 2.7 Prototype instrument (2005), from the present work

The inner workings of commercial Coriolis meters are closely guarded and the available information on the working of existing instruments is limited - mostly in the form of patents published by the respective inventors. The present work deals with innovations on several system aspects, together leading to better overall performance. These innovations are discussed in detail in subsequent chapters; they are presented here as a preview, contrasted against the state of the art in existing Coriolis mass flowmeters.

\subsubsection{Existing Coriolis flowmeters: state of the art}

The instrument shown in Figure 2.8 closely resembles the example U-tube instrument discussed in section 2.3. The tube is actuated in a "swing" motion by means of a solenoid and the motion of the tube is picked up by means of two voice coils, attached to the tube corners.

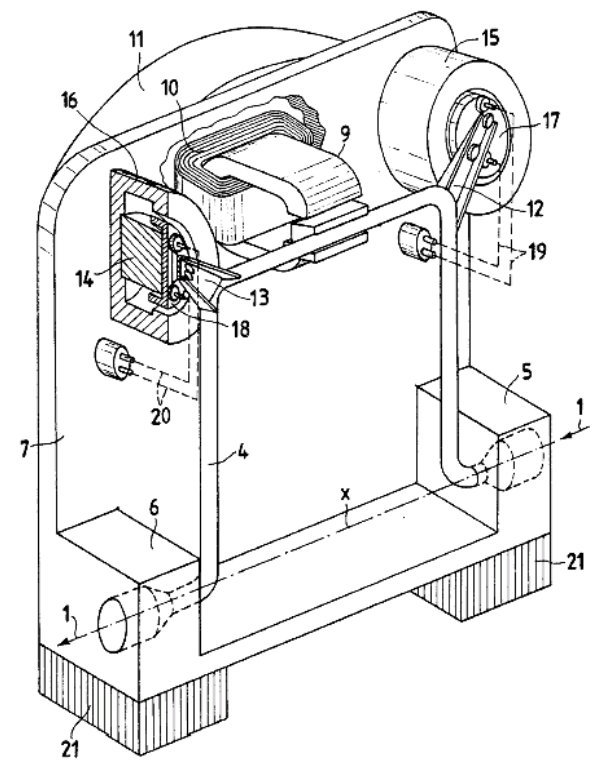

Figure 2.8 A swinging U-tube Coriolis meter - Patent: US 4895030 (Bergamini et al., 1990) 
This form has several disadvantages. Here the tube ends (fixed to the chassis at points 5 and 6 ) are away from each other. This structure is susceptible to distortion under a thermal gradient (a warm fluid medium). There are heavy and paddle-like (having large wind resistance) attachments upon the tube. Coriolis forces being in phase with damping forces; will be indistinguishable, and influenced by the windage.

The actuation is achieved by means of applying a force in the middle of the tube. If the force is not exactly in the middle, the tube will see it as a combination - force plus torque. Based on phase relations, most of this torque can be eliminated, but is nonetheless undesirable. The actuator is a solenoid $(9,10)$. Magnetization of the ferromagnetic components may cause outof-phase actuation (can be mistaken for Coriolis force).

In addition to the arguments mentioned in section 0 , the vertical limbs of the U-shape are subjected to bending, and the Coriolis force is generated in these very limbs. The Coriolis force, dependent on the local angular velocity will then be not uniform over the entire tubelength, but concentrated at the parts with the most deflection. Imagine now that the nature of bending changes slightly; this will affect the instrument sensitivity.

The sensors used to measure the position of the tube and thereby the Coriolis force, also see all the excitation motion. The (small) Coriolis motion is buried in a large common-mode signal corresponding to the excitation. This is avoidable.

This particular instrument consists of a single tube on a heavy chassis. The tube vibration will cause a chassis-shake. If the chassis is not sufficiently heavier than the tube, the chassis motion may be misinterpreted as Coriolis motion.

The form shown in Figure 2.9 has two tubes usually carrying exactly the same mass flow (as they are connected in series).

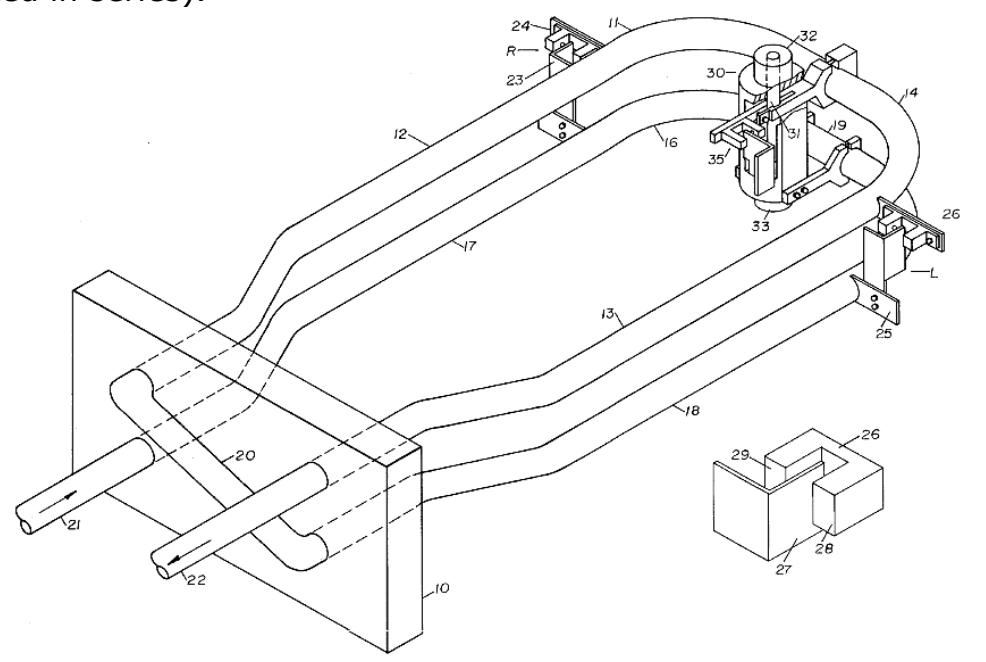

Figure 2.9 A twin-tube Coriolis meter - Patent: US 4192184 (Cox et al., 1980)

This configuration, though generally the same as the previous one, offers some advantages. The double tube reduces the problem of the chassis shake. Also, notice that the actuation and the position sensing is done as a relative measurement - between the two tubes; this makes external vibrations invisible (or less visible) to the position sensors. The numerous attachments for the purpose of actuation and sensing also seem smaller than the previous example.

For the rest, this instrument has all the drawbacks of the instrument in the previous example. Additionally, the double-tube construction has some problems - the assumption behind the 
twin-tube construction is that the two tubes are a mirror-symmetric in all respects. This assumption is not true, especially when the fluid medium causes a temperature difference in the two loops. The natural frequency of oscillation of one loop now differs slightly from that of the other, while they are forced to oscillate in tandem. The resulting changes in the excitation requirement can lead to drifts. The same problem may also result from an inhomogeneous medium inside the tube.
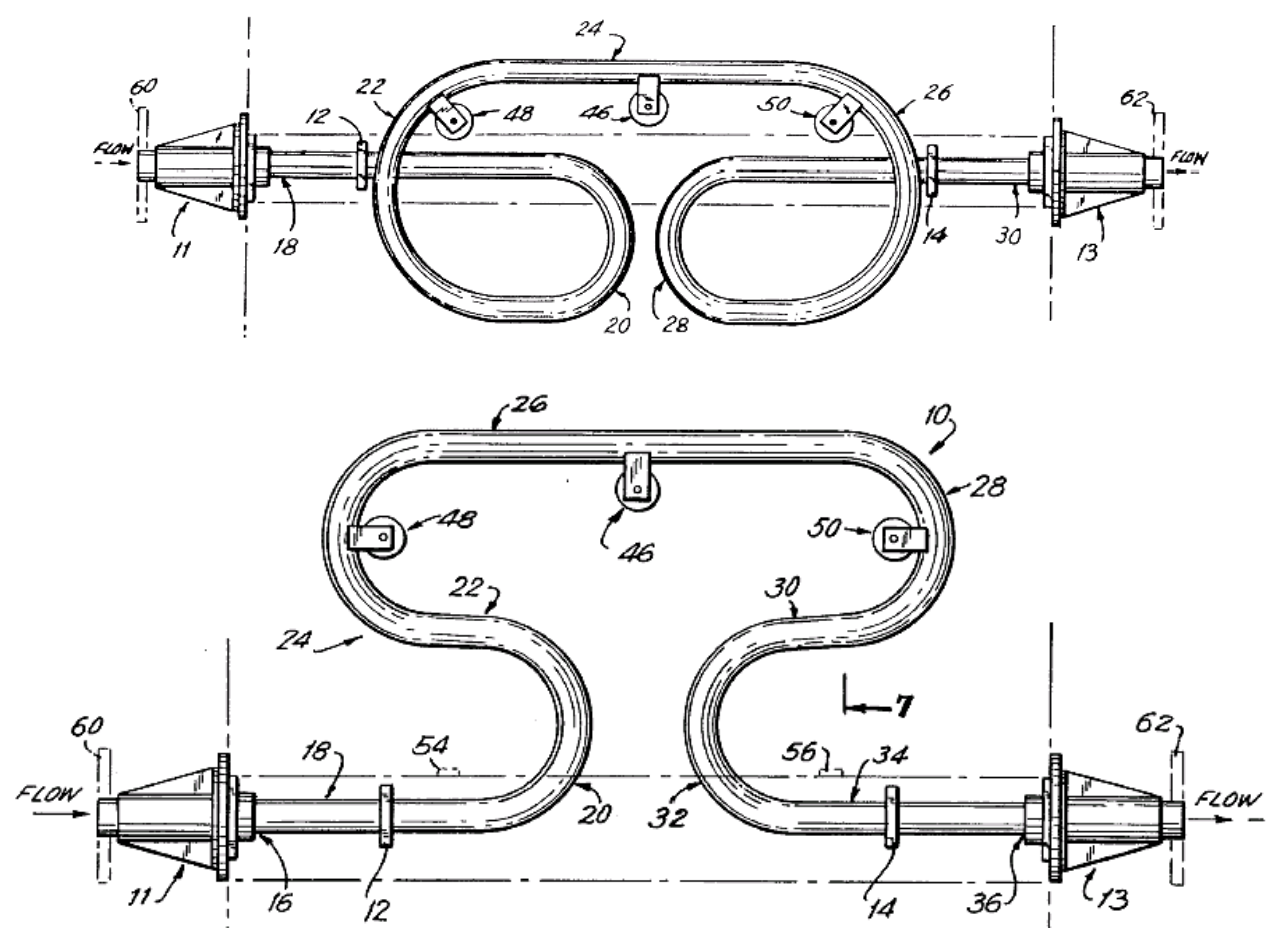

Figure 2.10 A folded tube construction - Patent: US 5044208 (Corown et al., 1991)

An improvement in these tube shapes (see Figure 2.10), also implemented as double tubes, is the positioning of the sensors nearer to the rotation axis of the excitation motion. The common mode (excitation) signal seen by the position sensors is somewhat smaller, resulting in a higher phasor sensitivity (discussed in Chapter 5) - which can be thought of as a mechanical gain. Here, errors originating from sensors, electronics and processing become relatively smaller. A problem that still remains is that the inlet and outlet attachment points are far from each other; this will probably cause temperature-dependent drifts. 


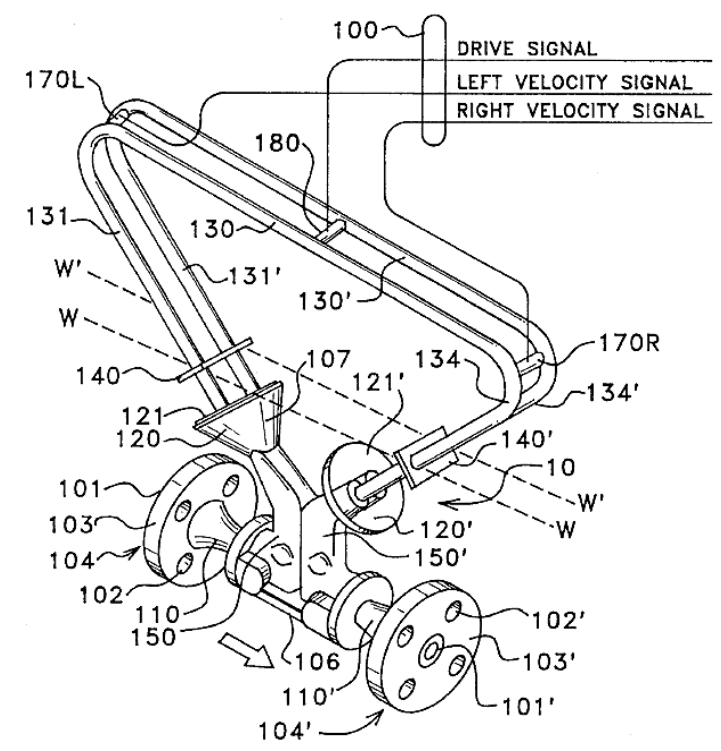

Figure 2.11 Attachment-points in proximity - Patent: US 6044715 (Ollila et al., 2000)

An improvement is seen in this example in the proximity of the attachment points. However, the interconnection plates (140) still probably influence tube interaction. In this example too, the position sensors see the entire excitation signal - meaning lower phasor gain and poorer use of the sensors' dynamic range for the Coriolis motion.

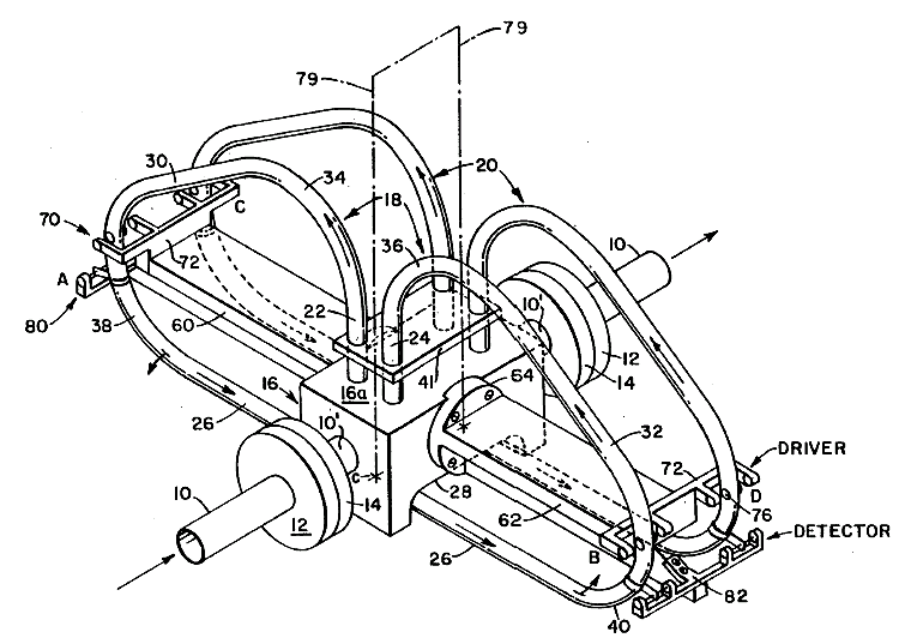

Figure 2.12 Folded tube with attachment-points in proximity - Patent: US 5050439 (Thompson, 1991)

Several improvements are seen in this example - the attachment points are situated close to each other. The option of a "twist mode excitation" is also discussed, whereby it becomes possible to place the position sensors very close to the rotation pole, thus improving the mechanical phasor gain. However, the excitation is still in the form of two separate (force) actuators (which may be mismatched - with drastic consequences), and there are still a lot of attachments upon the tube, for example (see patent text), the proposed LVDT - like sensing.

\subsubsection{The Coriolis tube from the present work}

The shortcomings illustrated in the previous examples have been judiciously avoided in the design of the design of the prototype instrument illustrated in Figure 2.13. 


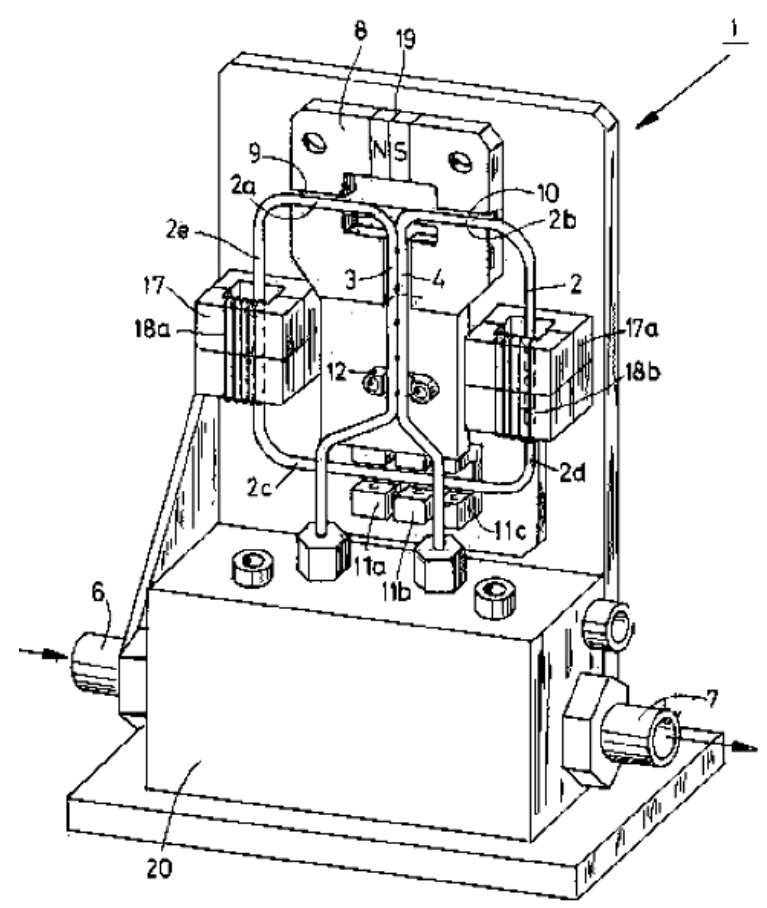

Figure 2.13 The prototype (Patent schematic) from present work

These are mainly in the areas of tube-shape $(\mathbf{T})$ excitation $(\mathbf{E})$ sensing $(\mathbf{S})$ and processing $(\mathbf{P})$ :

- (T) The moving tube is attached to the chassis at a single fixation point (12). Extra bent lengths of tubing between this fixation point and the fluidic couplings to the base block (20) allow the entire "sensor tube" to be in one plane, and act as strain-relief for the fixation point (against thermal \& constructional stresses at the fluidic couplings).

- $\quad(\mathbf{T}, \mathbf{E})$ The oscillatory excitation of the tube is in a twist mode. Beginning at the attachment point, the central pair of tubes acts as the (torsion) spring. This central section twists, (no bending) resulting in no Coriolis force here.

- $\quad(\mathbf{T}, \mathbf{S})$ The rectangle formed by the tube, on the other hand, does not deform much; the rotating sections of this rectangle generate Coriolis force in presence of mass-flow.

- $\quad(\mathbf{E}, \mathbf{S})$ Barring the fixation point and a vane for sensing purposes, there are no attachments upon the tube - actuation and sensing is done in a contactless manner.

- (E) The excitation is applied as a torque (and not as a force, or pair of forces). This means, that even with position misalignment, the nature of excitation stays constant (principle of translation of a torque).

- (S) The position sensing (of the Coriolis tube) is done close to the rotation axis, meaning a higher phasor gain.

Apart from these (visible) improvements, several other novel methods are employed for using the information from the oscillating tube:

- (S) Position sensing (of a straight line) is done with three sensors, 11a 11b and 11c. Though contra-intuitive, this allows measurement based purely on time-domain information. (discussed further in 5.4.2)

- (P) Measurement of Coriolis force is done in terms of phasor-angle differences. This is a difference-measurement, entirely in the time domain, making the gain \& offset calibration of (position) sensors unnecessary.

- (P) The Coriolis force is measured in terms of "amplitudes' ratio" rather than as a phase-shift or time difference, as described in literature \& patents. This prevents nonlinearity due to approximation in presence of large ( $>1 \mathrm{e}-2 \mathrm{rad}$ ) phasor angles.

- (E) Tube excitation based on position feedback allows the tube to oscillate at its natural frequency (eigenfrequency) - this allows density measurement and sensitivity compensation. 
- (P) The temperature of the tube is also measured. With knowledge about the material properties of the tube, correction (for temperature) is possible.

\subsection{Conclusion}

In this chapter, we have seen the fundamental working principle of Coriolis meters. We have seen a simple construction to generate Coriolis force from mass flow in a tube. In order to have a practical construction, an oscillatory source of rotation is seen to be advantageous (rather than a continuous rotation in one direction). The oscillatory sensor is then considered as a simple u-tube shape and several more complex shapes are illustrated along with the various advantages that they offer. The difficulties in measuring this force are discussed. The various drawbacks of existing tube shapes are identified and avenues, where there is room for improvement, are listed.

In Ch. 2.6.2, the innovative elements of the present work, namely the tube-fixation, oscillatory rotation excitation, tube shape, contactless sensing and torque-actuation, strategic sensor and actuator placement, multi-sensor time-domain measurement, and ratiometric detection are discussed. 


\title{
3 Requirements/Specifications
}

\author{
Target setting \\ The objective of the project behind this research was to develop a range of mass flow (rate) \\ meters, based on the Coriolis principle, and controllers based on it, to measure and control low \\ flow rates in liquids and gases. It was proposed to have a number of variants of the \\ instrument to cater to specific flow ranges, each variant measuring flow-rates a decade $(x 10)$ \\ smaller than the previous one.
}

\subsection{Functional specifications}

The functional specifications are typically the specifications that are presented in a 'product folder' to a customer who views the instrument as a black box. The functional specs may be subdivided into performance specifications (how well the instrument performs) and operational conditions specifications (what the instrument can withstand). Additionally there may be technical specifications for the dimensions, footprints, interfaces, appearances etc. The Functional specifications are a good starting point to enumerate the measurement steps needed to qualify the outcome of the project; the qualification tests and performance metrics (expectations) may be planned in advance.

Specifications are the result of requirements defined at the beginning of a project. In the following text, the functional requirements (FR) are enumerated, with an identifier number of the format $\boldsymbol{F R} \boldsymbol{x} \cdot \boldsymbol{y}$, where $\boldsymbol{x}$ is the serial number identifying the requirement and $\boldsymbol{y}$ is the instrument type number. Only those requirements that are relevant from a scientific standpoint are listed, together with a brief example or a description of the implications thereof.

\subsubsection{Performance specifications}

\section{FR 1 to FR 3: Flow range}

A wide range of flow rates is to be measured - the ratio between the smallest and largest (expected application full-scale) is about 1:1000. In order to better utilize the dynamic range it is proposed to distribute the flow ranges over multiple types of instruments:

\section{- FR 1.1 Type I- Flow range: $\quad 10 \ldots 200 \mathrm{~g} / \mathrm{h}$ full scale (FS) \\ - FR 1.2 Type II- Flow range: $\quad 100 \ldots 2000 \mathrm{~g} / \mathrm{h}$ FS \\ - FR 1.3 Type III- Flow range: $1000 \ldots 20000 \mathrm{~g} / \mathrm{h}$ FS}

In general, a Coriolis mass flow rate meter senses mass flow by means of translating the Coriolis force generated in a meter-tube into a "measurable quantity" - e.g. strain in a straingage or displacement in an elastic member. This (small) Coriolis force has to be measured in presence of (unwanted) disturbances that, in an absolute sense, scale with geometry (the larger the meter tube, the more the disturbance). This pushes the design in favor of a smaller construction. In contradiction to this is the pressure drop across the tube. The acceptable pressure drop across a commercial flow meter is about 1 bar, i.e. $1 \mathrm{e} 5 \mathrm{~Pa}^{13}$, while that across a controller is $\sim 3$ bar (3e5 Pa). The pressure drop $\Delta P$ in a fluid, of density $\rho$ and viscosity $\eta$, flowing through a tube having a diameter $d$ and length $L$, may be determined by the Hagen Poiseuille equation (see section 5.1.1). In general, the smaller the tube, the larger is the pressure-drop, for a given flow-rate. This pushes the design in favor of a larger tube diameter.

In the requirements FR1.y above, two values are specified for each type of instrument. For example, in the type-I instrument, the smaller of the two (i.e. $10 \mathrm{~g} / \mathrm{h}$ ) is intended for gases

\footnotetext{
${ }^{13}$ This comes about as the commonly available driving-pressure in a fluidic system is about 10 bar. A 1 bar drop across the meter corresponds to $10 \%$ of this supply-pressure. In flow controllers, for rapid control, it is essential that more than $50 \%$ of the pressure drops across the control-valve (see [10]); the 3 bar specification for controllers accounts for this extra drop across the valve. See also: FR 14
} 
(typ. air) while the larger value $(200 \mathrm{~g} / \mathrm{h})$ is intended for liquids (typ. water). This is for the practical reason, that with the allowed 1 bar pressure drop, the ratio of mass flow-rate of air (a typical gas) through a meter tube and the mass flow rate of water (a typical liquid) through the same tube corresponds to the ratio of these two values.

\section{- FR 2.0 Dynamic range in relation to the Full Scale (FS) (meter): $1: 1000$ - FR 3.0 Lowest controllable flow in relation to FS: (controller) $1: \mathbf{5 0}^{14}$}

The dynamic range is the ratio of the smallest to the largest quantity that an instrument can measure, and is practically determined by various factors such as the measurement noise, digitizer resolution etc. As the full-scale flow-rate for each type of instrument spans a range of $1: 20$ (e.g. FR1.2 of the type II instrument is specified to be 100 to $2000 \mathrm{~g} / \mathrm{h} \mathrm{FS}$ ), if the dynamic range specification is satisfied for the lowest rate (i.e. $100 \mathrm{~g} / \mathrm{h}$ ), it will be a factor 20 better at the highest rate (for the same instrument). This implies the "best case" dynamic range to be $1: 20000^{15}$.

\section{FR 4 to FR 6: Measurement error}

The meter is expected to meet the following values after factory calibration and also an on-site "Auto-Zero" (offset correction step):

- FR 4.0 Zero stability: better than $0.1 \%$ of max. FS

E.g. for type I: $0.2 \mathrm{~g} / \mathrm{h}(0.1 \%$ of $200 \mathrm{~g} / \mathrm{h})$; If the type I meter is operated with zero actual flow, then under various environmental conditions (temperature, pressure, humidity, medium properties etc.), the absolute of the averaged (over one second) reading reported by the instrument should be less than $0.2 \mathrm{~g} / \mathrm{h}$.

\section{- FR 5.0 Totalizer error: $<0.1 \%$ reading + time $\times(0.1 \%$ FS $)$}

E.g. If a type I instrument (having FS: $200 \mathrm{~g} / \mathrm{h}$ ) is operated for 1 hour and the actual net flow through the instrument is $100 \mathrm{~g}$, then the totalizer reading must be within:

$$
100 \pm(\underbrace{\frac{0.1}{100} \cdot 100}_{0.1 \% \text { reading }}+\underbrace{1 \cdot \frac{0.1}{100} \cdot 200}_{1[\mathrm{hr}] \times 0.1 \% \mathrm{FS}} \text { [g], i.e. between } 99.7 \mathrm{~g} \text { and } 100.3 \mathrm{~g}
$$

\section{- FR 6.0 Uncertainty in the reading "instantaneous": No requirement}

As it is not easily possible to measure the actual instantaneous flow rate, there is no requirement on the instantaneous reading. This requirement is listed here for clarification only.

\section{FR 7, FR 8: - Behavior in the time domain}

- FR 7.0 Response time (meter): t63\% $\leq 0.2 \mathrm{~s}$

This specification assumes that the meter follows a 1st-order (exponential) tracking of the actual flow. (In a $1^{\text {st }}$ order system, in one time-constant $\tau$, the reported value traverses $63 \%$ of the applied step change.) When specified in this manner, the instantaneous measurement noise (usually much smaller than the remaining $37 \%$ at the end of one time-constant) does not significantly affect the determination of this time-constant.

\section{- FR 8.0 Settling time (controller): t98\% $\leq 1$ s (0 .. 100\% setpoint)}

When making a setpoint-step, the actual flow-rate $1 \mathrm{~s}$ after issuing the step command should have settled to within $+/-2 \%$ FS of the final value. E.g. for a type II instrument, with flow-range (Full Scale) set to $100 \mathrm{~g} / \mathrm{h}$, the value $1 \mathrm{~s}$ after a set-point step should be within $+/-2 \mathrm{~g} / \mathrm{h}$ of the final value.

\footnotetext{
${ }^{14}$ The dynamic range (turn-down) of the control-valve is the limiting factor

15 This is an improvement by a factor 20 over commercially available instruments
} 


\subsubsection{Operational conditions specifications}

While not as visible as the performance specifications, the operational condition specifications play an important role in the design choices and choices of operating principles. If neglected at the outset, these may lead to expensive redesign \& delays during later stages.

\section{FR 9 to FR 11: Temperature}

\section{- FR 9.0 Ambient temperature range: -10 to $+70^{\circ} \mathrm{C}$; sterilization (fluid at $150^{\circ} \mathrm{C}$ ) for 15 minutes}

Survivability: The said temperature range precludes the use of certain materials, e.g. plastics and certain types of permanent magnets. The power dissipation budget of the instrument too should consider the upper temperature. The lower temperature range does not apply to media like water that would freeze, thereby possibly damaging the instrument.

Function: The ambient temperature has an effect on pickup-sensor characteristics and on (tube) material properties (see [9]). The impact of the wide $\left(80^{\circ} \mathrm{C}\right)$ temperature range on the performance should be accounted for.

\section{- FR 10.0 Rate of change of instrument temperature (survivability): $1^{\circ} \mathrm{C}-$ per-second}

- FR 11.0 Drift due to change in fluid medium temperature: $\pm 0.01 \%$ reading $/{ }^{\circ} \mathrm{C}$ The drift is to be interpreted as a linear parameter, which means that after calibration at the instantaneous temperature of e.g. $20^{\circ} \mathrm{C}$, for a rise to e.g. $30^{\circ} \mathrm{C}$ an error in the instantaneous reading of $10 \times 0.01=0.1 \%$ is allowed. This is to be added to the sum of previously mentioned errors.

\section{FR 12 to FR 17: Pressure and media properties}

- FR 12.0 Fluid types: All clean, homogeneous fluids compatible with AISI 316 AISI 316 stainless steel is the material of choice for typical applications of Coriolis mass flow rate meters (semiconductors, medicines, foods \& beverages etc.). This seems a roundabout way of specifying that the Coriolis meter tube should be made of a material that can withstand media that are a superset of media that AISI 316 can withstand. The material choice is limited to AISI316 itself, and a few others like titanium and Hastelloy. Materials such as Kalrez may be used in couplings.

- FR 13.0 Viscosity: Up to 0.1 Pa.s

The nominal viscosity of water is $0.001 \mathrm{~Pa} \cdot \mathrm{s}$. This is considered when specifying pressure drop over the instruments. It is understood that fluids with higher viscosity shall not achieve the rated flow-per-pressure-drop specification.

- FR 14.0 Pressure drop: < 1 bar at nominal water flow (meter); 3 bar (controller) From experience (see [10]), it is recommended that in order to achieve specified speed in a controller, the pressure drop across the control valve should be at least $50 \%$ of the total pressure drop for liquid media (and $>75 \%$ for gases).

- FR 15.0 Pressure sensitivity: $\leq 0.01 \%$ reading per bar input pressure Similar to FR 11.0, this allows for errors due to minor deformations in tube-geometry due to fluid pressure.

- FR 16.0 Maximum system pressure: 100 bar The tube should survive and operate normally at this pressure. This specification will dictate the minimum wall thickness of a particular tube.

- FR 17.0 Density measurement error: $<10 \mathrm{~kg} / \mathrm{m}^{3}$

The density of the medium affects the inertia of the Coriolis tube and can be inferred from instrument variables, primarily the eigenfrequency of oscillation of the tube. The 
density should be measured with an error smaller than $10 \mathrm{~kg} / \mathrm{m}^{3}$. A two-point calibration may be carried out per instrument, using two reference fluids (typ. water and air) having known densities.

\section{FR 18 to FR 21: Shocks and Vibrations}

Due to the lack of a precedent, the vibration specifications were difficult to quantify at the beginning of the project; the following are refinements made "on the fly" during the execution of the project.

- FR 18.2 Harmonic vibrations (Example - for type II instrument): $<10 \mathrm{~nm}$ at \{eigenfrequency $\pm 10 \%$ \} of tube excitation eigenmode $<100 \mathrm{~nm}$ at \{eigenfrequency $\pm 10 \%$ \} of tube "response" eigenmode No performance can be guaranteed in case of harmonic vibrations of the mounting in the frequency range around the excitation frequency, because resulting accelerations cannot be distinguished from those caused by the Coriolis effect. In case this occurs, vibration isolation or tuning of the mounting is to be performed. The restriction at the response eigenmode is to prevent the tube being excited with large amplitude, thereby exceeding (internal) sensor limits (see section 6.1.2)

- FR 19.0 Random vibrations: <1e-3 m/s at frequencies below $500 \mathrm{~Hz}$ Only a fraction of the energy of the wideband spectrum occurs in the disturbing frequency range. This level can be higher than harmonic vibrations, as no resonance occurs.

- FR 20.0 Shock (operational):

In case of a shock disturbance the measured value should return to its original position within 2 seconds. This requirement does not apply when the shock induces a resonance of the foundation in the frequency range around the excitation frequency.

- FR 21.0 Shock (survivability): $1000 \mathrm{~m} / \mathrm{s}^{2}$

The instrument should survive shocks with peak accelerations in excess of $1000 \mathrm{~m} / \mathrm{s}^{2}$ (i.e. about $100 \mathrm{~g}$ ) without need for re-calibration. This value is the typical acceleration encountered when the instrument is dropped on a massive and hard surface.

\subsection{Technical specifications}

The technical specifications are presented to a design engineer who, based on this information makes appropriate design choices for the instrument. The technical specs may be subdivided into three main sections

- Mechanical

- Electrical

- Software

In order to make an appropriate technical specification, some information regarding the tasks to be accomplished is essential. This may come in the form of experience regarding the needed tasks in comparable previous instruments, and from proof-of-principle tests (PoP) done in the lab on various subsystems prior to making a specification.

Prior experience was available at the beginning of this research in the form of other Coriolis effect mass flow-rate meters. Additionally, several people (among which were also graduate students from the University of Twente) did a lot of lab-tests in the early stage of the project. Based on the experience and the knowledge learned from these two sources, the following technical specs have been put forth. As some of the specifications are not relevant from a scientific viewpoint, the items listed here are a subset of the actual technical specifications list. Similar to the FR numbering, the technical specifications too take on a $\boldsymbol{T R} \boldsymbol{x} \cdot \boldsymbol{y}$ format

\subsubsection{Mechanical specifications}

For interoperability, it is desirable to have the mechanical and fluid interfaces of Coriolis instruments similar to other existing flow-meter instruments. Apart from the previously mentioned materials' requirement and the obvious cosmetic aspects, the following specifications are noteworthy: 
- TR 1.0 Tube Eigenfrequencies:

For various reasons (discussed in chapter 4 ) it had been decided to employ a Coriolis tube oscillating in an eigenmode to generate and measure Coriolis force. The so-designed tube will have many other eigenmodes, at least one of which will be covered by the motion that results from the Coriolis force. For reasons also discussed in chapter 4, these two 'primary' eigenmodes must have frequencies substantially away (factor 1.5) from one another. The instruments are to be used in an industrial environment, with the presence of mainsfrequency interference (mechanical vibrations, electrical conduction ripple and radiation, optical flicker, etc.) To avoid influence of these, it is desirable to have the excitation frequency (this is the frequency upon which the Coriolis force is "modulated") far away from the mains frequency and its harmonics (especially the second, third and fifth - the most dominant). Two things should be taken into account: (1) the tube eigenfrequency is not constant; it varies substantially with the density of the fluid medium. Typically, $5 \%$ to $8 \%$ variation in eigenfrequency due to density was observed in PoP instruments. It should be away from the mains frequencies and harmonics under all conditions with a margin of $5-10 \mathrm{~Hz}$ and (2) the instrument will be used internationally; the mains frequency may be $50 \mathrm{~Hz}$ or $60 \mathrm{~Hz}$.

\section{- TR 2.0 Tube Eigenmodes:}

As discussed in chapter 4 and TR1.0, the Coriolis tube will have several eigenmodes. These typically result from a mass-spring system, where one portion of the tube provides the mass, while another provides stiffness (the spring). It is possible that a mode is formed by a 'chain' of masses and springs - foundation-spring1-mass1-spring2-mass2-spring3-foundation.

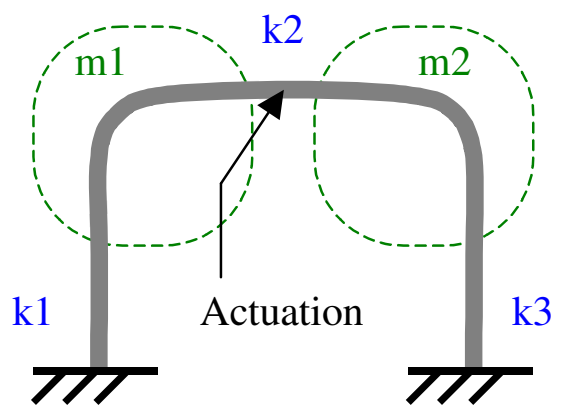

Figure 3.1 The simple U-shaped tube - the stiffness and inertia of the sections of the tube are simplistically discretized

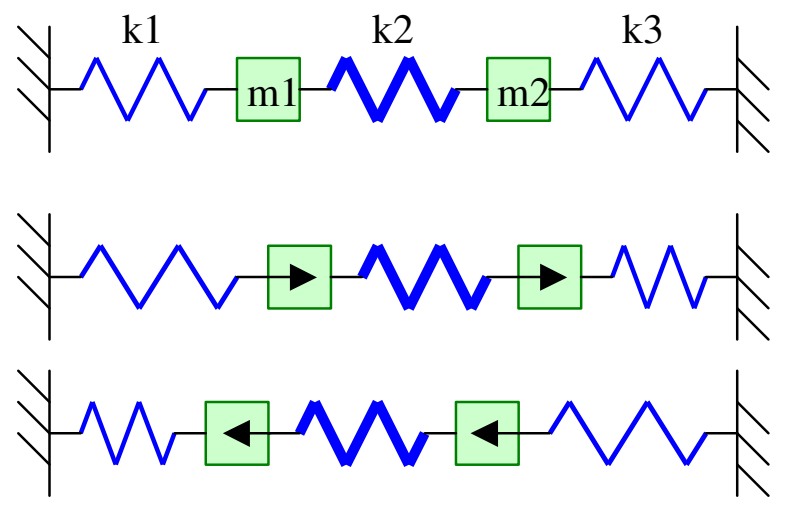

Figure 3.2 A resonant (1 DoF) system formed as a 'chain' (see text)

If the middle spring (spring $\mathrm{k} 2$ in this example) is quite stiff, and the ratios mass 1 :spring 1 and mass 2 :spring 3 are similar, then the two masses will be forced to move in a synchronized manner by the stiff spring $\mathrm{k} 2$. Now, if the stiffness and/or masses were to change slightly in the presence of small (unknown and unavoidable) environmental variations, the exact motion 
of the masses in such a construction would slightly non-symmetric and hence unpredictable. Hence, such a 'chain' should be avoided for the excitation mode. The primary energy-storingelement pairs $(\{\mathrm{k} 1, \mathrm{~m} 1\}$ and $\{\mathrm{k} 2, \mathrm{~m} 2\})$ should be combined into a single physical pair $(\{k, m\})$ if possible.

\section{- TR 3.0 Chassis Eigenfrequencies:}

The vibrating Coriolis tube will be mounted on a chassis, which should ideally appear as an infinitely stiff support for the tube. If not, the modes introduced in the chassis might adversely affect (interfere with) the measurement of motion of the tube. Practically, the chassis should be such that its lowest eigenfrequency should be several times higher than the excitation frequency of the tube.

\section{- TR 4.0 statically determined construction:}

Due to non-ideal construction (e.g. unavoidable constructional asymmetries), there always exists coupling (leakage of power) between eigenmodes. In presence of damping, (leading to phase-shift), such leaked power can result in forces indistinguishable from Coriolis forces. It is possible, from experience from PoP tests, to calibrate-out a small amount of leakage.

However, for the calibration to be effective, the imperfections must stay constant over time. A statically determined construction (see [3]) is essential. Furthermore, the fixed ends of fluidcarrying tube(s) should be as close to each other as possible, avoiding mechanical 'bridges' in order to minimize deformation of the tube in the event of (unavoidable) thermal gradients.

\subsubsection{Electrical specifications}

\section{- TR 5.0 Digital or analog processing:}

In previous PoP studies (see [9]) it has been demonstrated that digital processing is feasible for processing sensor signals to translate them to a flow measurement. Due to advancement in analog to digital signal converter and processing techniques in recent years, especially due to the maturation and widespread use of PC audio, analog signals can be represented and processed in the digital domain with high resolution with reasonably low cost and effort. A digital system offers flexibility (e.g. internal rerouting of signals and tweaking of functional blocks), transparency for debugging and observation of system behavior, upgradeability of features, robustness (the travel-path of analog information is much shorter, and consequently, less susceptible to external disturbance or internal noise). A few COTS digital parts can emulate the function of several specialized analog parts, making a digital system attractive. If desired, a layered approach can be taken towards the engineering of the digital processing, where several sub-iterations of the "V-model" can be made without time-consuming rewiring of analog parts. The details are dealt with in section 3.2.3.

- TR 6.0 Power consumption and heat dissipation:

For compatibility reasons, and dissipation possibilities (ability to get rid of generated heat), it is a requirement that the instrument, as a whole, should not consume or dissipate more than about 8 Watt power. This translates to about $333 \mathrm{~mA}$ current consumption at 24V supply voltage. (Note: As the meter does not export any power, almost all consumed power is converted to heat in the instrument.)

In a particular PoP implementation, it has been demonstrated that in order to have the Coriolis tube oscillate with sufficient amplitude, it needs about $6 \mathrm{~mW}$ of mechanical power to sustain oscillations. This value will of course change with a particular form or size of tube used. However, this specification gives an order-of-magnitude for the requirement.

\section{- TR 7.0 Uncertainty due to time reference:}

It has been demonstrated from calculations and a set of PoP instruments that an achievable amplitudes' ratio between the excitation motion and the response motion due to Coriolis forces is about $100: 1$. At the smallest resolvable flow $(0.01 \%$ of the nominal) this ratio leads to a phase-shift (between sensor signals) that corresponds to a time-delay to the order of about 1 ns. A jitter in the clock signal will lead to noise in the measurement. The details of the effects 
of clock jitter and inaccuracy are dealt with in chapter 5.4.3. The SONET ${ }^{16}$ specification, for example, defines Jitter as "the short-term variations of a digital signal's significant instants from their ideal positions in time". Clocks jitter in the order of picoseconds is achievable with commercial clock generators. Relative error of such clock generators, on the other hand, is usually a few PPM. Both of these are several times better than required.

\section{- TR 8.0 General sensor quality requirements:}

Position sensors are to be used to determine the motion of the Coriolis tube to analyze it for determining the extent of the generated Coriolis force. For the same reasons as in TR 7.0, it is crucial that these motion sensors have as small time delays as possible; in the worst case, they should be matched with each other. The estimate for allowable variation (over time) in delay mismatch is a few tens of nanoseconds. Matching the gain of each sensor is not critical, as a ratiometric and time-referenced measurement is adopted.

- TR 9.0 Galvanic isolation \& current through the instrument:

If physical principles are employed in the CMFM that make it susceptible to stray current return paths, especially in industrial environments, the sensitive components must be galvanically isolated from such paths. Typically, a PoP instrument employed a Lorentz actuator for excitation of the meter tube. A stray (unwanted) $50 \mathrm{~Hz}$ current flowing through this tube would create indeterministic forces that may interfere with the meter principle.

\subsubsection{Specifications dictated by Software}

As mentioned in TR10.0, the meter (soft) function, the communications interface and calibration intelligence is to be handled by a separate proven processor unit. This primarily leaves over the core functionality of:

- Excitation of the tube at a predetermined amplitude

- Determination of amplitudes' ratio by analyzing the effect of Coriolis force on the motion of the tube

And secondarily

- The determination of the (natural) frequency of oscillation of the meter tube

In previous PoP studies, the meter functionality has been implemented and tested on an industrial PC platform; the software that drives this PoP gives insight into the needed processing power. The software on the PoP platform was structured in a manner so as to be driven by timer interrupt ticks. Each "time slice" (time between two successive interrupt "ticks") has the full functionality - input + processing + output - contained within it. This functionality is repeated at the rate of timer ticks. This means, that if a target CPU is able to perform all tasks that are in one "slice" within one time-slice in the PoP setup, it is sufficient for the required operation.

\section{- TR 10.0 Processing power:}

Determination of needed processing power:

All the instructions executed in one time slice in the PoP are collected together. In case an operation runs in a loop, the instructions within that operation are repeated the number of times that the loop repeats. [The instructions running in the time-slice on the industrial PC in the PoP were coded directly in (5x86) assembly. Most of the instructions were coded as FPU assembly, (which is surprisingly powerful and non-tedious) on a 5x86 platform]. Next, the instructions are categorized by complexity of implementation. As the final target platform (i.e., the processor on which the instrument firmware shall be implemented) is probably not a $5 \times 86$ processor, the PoP instructions are factorized in terms of possible instructions on a candidate target processor. As an illustration, the following equivalence may be drawn:

\footnotetext{
${ }^{16}$ Short for Synchronous Optical Network, a standard for connecting fiber-optic transmission systems. SONET was proposed by Bellcore in the middle 1980s and is now an ANSI standard.
} 


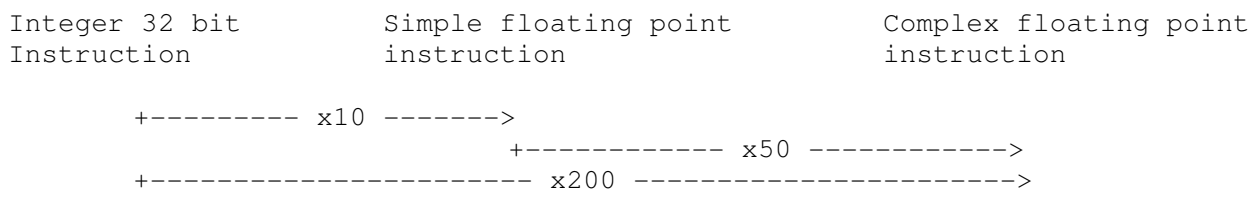

Estimated

Emulation

Penalty

Simple floating point

instruction
Complex floating point

instruction

In this illustration, 'integer' 32-bit instructions include operations like data move, integer addition, counters, etc. The 'simple floating point' instructions include instructions like multiply, divide, add, subtract, compare etc. The 'emulation penalty' gives a multiplier, which says, for example, that emulating a simple floating point operation on a processor by executing integer 32-bit instructions alone will incur a 10x penalty, i.e., 10 integer instructions will need to be executed to achieve the same function as a single 'simple floating point' type instruction. 'Complex floating point' instructions include operations like square-roots, logarithms, exponents and trigonometric operations. The 'penalty' for these is not the same, and usually not even deterministic (an arctangent operation, for example, may take more or less time depending on the value being processed). The stated penalty is only representative. Furthermore, while the penalty of carrying out a complex operation on a 32-bit integer processor (for example, the ARM-7) is a factor 200, the penalty on a processor that supports 32 -bit floating-point instructions (like the ADSP $21 \times x x$ ) is only a factor 50 . Based on these or actual measured values, a processing budget table for one time-slice may be drawn up:

(An illustration: ADSP21260 at $100 \mathrm{MHz}$ )

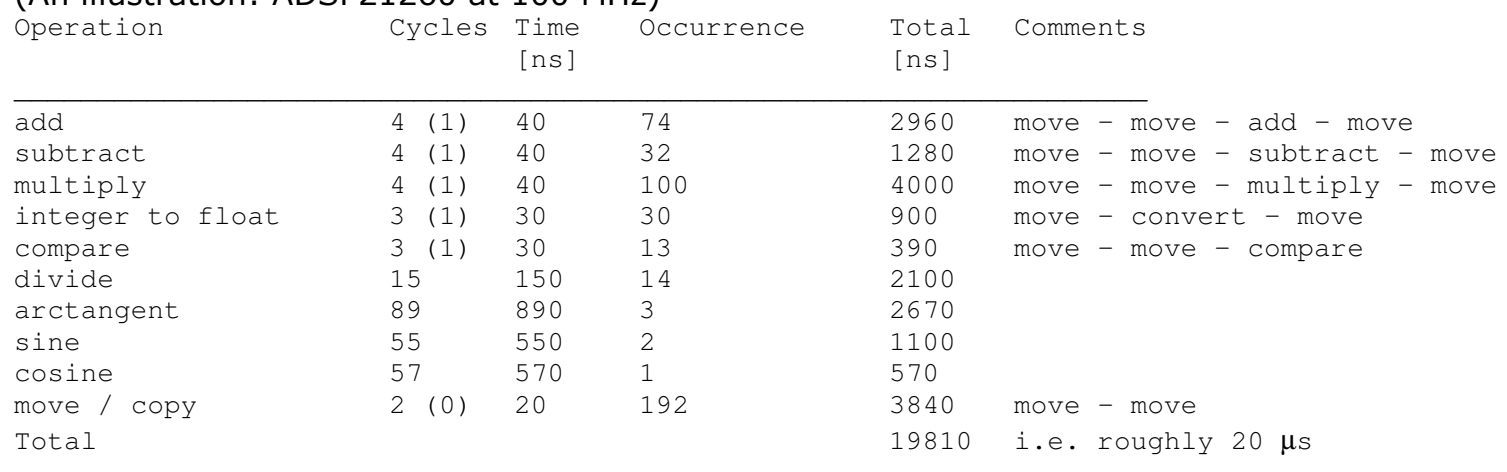

From the PoP, it has been demonstrated that a slice-processing rate of $5 \mathrm{kSps}$ (five thousand slices per second) or better is suitable for operation of the "core functionality". This means that each time slice should be $200 \mu$ s or shorter. In the example above, the used time is about a tenth of the available time. Experience says that in view of

- The decreasing cost of processing power

- The increasing cost and possible risks of reengineering

It is wise to choose a processing platform with at least $80 \%$ "head-room" - i.e. the estimated processor usage should be only $20 \%$ of the available processing power. In the example above, this 'wisdom' is heeded. Note here that by using simple optimization, it is possible to reduce processing. Using multi-rate processing, for instance, low frequency parts of the sensor algorithm can be executed fewer times per second than other parts. Alternative methods with similar functionality but easier embedded implementation, for example a FIR filter that is easy to implement on a DSP, or a 'sine' function implemented as a lookup table, also give reduced requirements. These are, however optimizations, and ...premature optimization is the root of all evil ${ }^{17}$.

To summarize, in terms of "simple floating point" operations, the target processor needs to execute about 2000 instructions per time slice and should be capable of executing at least $5 \mathrm{x}$

\footnotetext{
17 "We should forget about small efficiencies, say about $97 \%$ of the time: premature optimization is the root of all evil." (Knuth, Donald. Structured Programming with go to Statements, ACM Journal Computing Surveys, Vol. 6, No. 4, Dec. 1974. p.268.)
} 
as many. Measurements or simulations on candidate target processors to satisfy this condition takes away guesswork and prevents the risk of hardware redesign.

- TR 11.0 Processing precision:

From PoP tests, it is seen that the processing precision of 24 bits $^{18}$, and the high dynamic range due to an 8-bit exponent, offered by the IEEE 754 32-bit floating point representation is sufficient to carry out trigonometric calculations and implement IIR digital filters for phasedetection. As such, a typical 32-bit signal-processor (with a 32 bit FPU) is recommended.

\subsection{Testing methods}

Generally speaking there are four methods ${ }^{19}$ (see [11]) to verify (prove) a requirement:

- Testing: prove compliance by means of a well-defined procedure.

- Analysis: prove compliance by means of calculations.

- Demonstration: prove compliance by means of a demonstration, for qualitative requirements.

- Inspection: prove compliance by means of studying the product, for visible design features.

Each of the functional requirements defined above is to be proven by means of testing. Most of the technical requirements need not be tested, but can be proven by means of inspection or demonstration. The test methods (TM) are summarized below with an identifier (number)

\section{TM 1.1 1.2 and 1.3: Range}

The measurement must determine the flow range in which the (stationary) error requirements are fulfilled. These requirements are to be measured for a number of flow rates within each of the flow ranges of the instrument, using weighing scales as a reference.

\section{TM 2.0 Error and uncertainty}

The static zero-error (offset) of the reference instrument is to be nulled beforehand The zero stability is to be measured without flow, under normal process conditions: with filled tube, under pressure.

Test practices:

- Sensitivity measurements are to be done from 0 to $100 \%$ of full-scale flow with $25 \%$ steps. Before the measurement the zero offset is eliminated. The dominant error is usually not the non-linearity, but a shift of the sensitivity line.

- Errors and repeatability are related to the totalizer, defined over 1 hour measuring time with constant flow and a 'flying start' and also a 'flying stop'. The actual measuring time depends on the flow rate and the measurement range of the scales: for a smaller flow it will take more time to achieve a measurement having a comparable relative error due to the readout of the scales.

- Meter noise or jitter causes high-frequency fluctuations of the readout. These may be suppressed by filtering of the measured data, e.g. by an exponential smoothing filter (simple first order digital filter), as long as this filtering does not conflict with other requirements. Since the above definition is related to the totalizer reading a constant flow, fluctuations or filtering do not affect the totalizer reading, as the measurement interval is much longer than the filter time-constants.

- The scenario for measuring the repeatability has not been specified. For the flow meter this may be defined as doing a statistically significant number of measurements for a fixed flow at realistic time intervals. As the repeatability applies to the totalizer at full flow, it is tested at $100 \mathrm{~g} / \mathrm{h}$ for a period of 1 hour.

${ }^{18}$ IEEE 754, Single-precision 32-bit representation: 23 bit fraction +1 implicit bit

${ }^{19}$ MIL-STD-961E 


\section{TM 3.0 Temperature}

Ambient temperature testing requires a temperature-controlled environment. A programmable environment chamber shall be used to create predefined temperature ramp for dynamic temperature tests. A reference temperature sensor shall monitor the environment temperature to qualify the built-in temperature sensors.

\section{TM 4.0 Pressure}

- Measurements on pressure drop can be performed with water, at normal system pressure.

- As the minimum system (over)pressure is 3 bar, and the instrument pressure-drop is about $1 \mathrm{bar}$, an additional resistance (e.g. a partly closed valve) will be needed downstream.

\section{TM 5.0 Response and settling time}

- To be measured for a number of different steps, for both increasing and decreasing flows.

- To be measured at minimum specified system pressure, because this is the most critical (worst-case).

- As the performance is not related to measurement error, no accurate reference instrument is needed; the output of the tested instrument itself can be used. Internal filtering is to be switched off where this affects the speed of response.

- The device used for switching during the response time measurement may not add any capacity, resistance or time lag.

- Before the settling time measurement the valve must be bled form air possibly trapped inside.

\section{TM 6.0 Vibrations}

- All vibration and shock tests are to be performed on a softly suspended rigid platform that can be given a defined acceleration in any translational or rotational direction. Harmonic or random vibration can be applied by means of an excitation coil; shock excitation by means of a hammer pendulum with well-defined tip stiffness.

\section{TM 7.0 Immunity for medium density}

- Is to be tested using different media (water, IPA, air) in a thermally well-conditioned environment using accurate scales as a reference, with sufficient measurement (totalizer) time. 


\section{Concept choice}

\section{Target setting}

The concept choices in the course of this project were, to a large extent, based upon proof-ofprinciple studies, simulations and 'thought experiments' carried out since 2001. Aspects of Coriolis mass-flow rate meters published in patents $\&$ other literature are used as a benchmark - a comparison for concepts implemented or chosen in this particular project.

\section{Peculiarity of the measurement principle}

Coriolis mass-flow rate meters, those tested in the PoP and also the vast array presented in various patents shows a peculiar parallel to a well-known measurement instrument, the moving-coil galvanometer; here, the Lorentz force is the cross product of current and magnetic field, whereas in Coriolis force is the result of the cross product of the mass flow and angular velocity vectors. Being based on an active measurement principle, a CMFM thus necessarily has moving parts. The analogy is as follows:

Galvanometer:

- Tiny current flows through coil

- Multiple turns increase sensitivity (force per unit current)

- Coil is strategically placed (in a piece-wise linear magnetic field)

- Force (or torque) causes the coil to move perpendicular to the flow and field

- Pushes a spring with a stiffness $k$

- $\quad$ Needle moves following $\bar{F}=k \cdot \bar{x}$ or $\bar{x}=\frac{\bar{F}}{k}$

- Sensitivity is thus also determined by stiffness

- Weak spring helps to increase sensitivity

- $\quad$ Spring must have constant stiffness and magnetic field must have constant flux density and coil must have constant number of turns for all allowed values of current if the meter is to be linear.

- Nothing else (other than the Lorentz force) should move the coil

Coriolis Mass flow rate meter:

- Tiny flow through tube

- Single turn for simple construction and low pressure drop

- Tube is strategically placed in a 'rotation field'

- DC rotation field is difficult to implement, hence AC rotation field is used

- The Coriolis effect generates a (Coriolis) force that relates the bias (AC rotation field) and the response (AC deflection) depending on the mass-flow rate.

- The Coriolis force deflects spring to an extent $\frac{1}{k}$ (or mass to an extent $\frac{1}{\mathrm{~m} \cdot \mathrm{s}^{2}}$, depending on implementation) hence lower stiffness (or lower mass) results in larger deflection.

- External actuation is essential to create a 'rotation field'; this actuation may not interfere with the instrument response (analogy: in a galvanometer, the coil itself may not be moved by the magnetic field, and hence is designed using non-magnetic materials)

- Parasitic couplings must be minimized; nothing else (other than the Coriolis force) should deflect the tube

This analogy helps to make some concept choices. The challenge in Coriolis meters lies in:

- How to generate a defined angular velocity or how to make measurements insensitive to unknown variations in the angular velocity

- How to measure a periodic (oscillatory) force

- How to make (force) measurements that are insensitive to variations in sensitivity of the force sensor 
- How to prevent other mechanisms from generating forces that may be mistaken for Coriolis force

Choosing a proper concept minimizes the costs and complications involved in the need to use excessively fine mechanisms and tuning and calibration procedures.

\subsection{Size shape and form of the sensing tube}

As discussed in chapter 2.3 (see also [12]), it is advantageous that the rotation leading to the generation of Coriolis force is periodic, i.e. oscillatory instead of continuous 'turning'. The oscillations can be, in principle, any arbitrary motion. However, excitation at an eigenfrequency of a suitable natural mode having a high Q-factor offers several advantages (see [9]).

To recapitulate the advantages of choosing a suitable oscillation eigenmode from [9]:

- The higher the Q-factor, the smaller the excitation force required to oscillate the tube

- An appropriate oscillation mode can be chosen such that the excitation and response are orthogonal modes. It is not necessary to fully know the magnitude of excitation; the motion caused by the Coriolis force is by nature distinguishable from the excitation itself

\subsubsection{The Q-factor}

A guitar string, a tuning fork, an oscillator crystal, and a clock pendulum are commonly known resonant systems. The oscillation of a resonant system is characterized by the constant exchange of stored energy between two types of energy storage elements [1]. In mechanical systems, the two energy types are usually kinetic energy (mass with a velocity) and potential energy (spring with a deflection <<tuning fork >> or weight with a height $<<$ pendulum $>>$ ). Energy is conserved (the pendulum keeps swinging unless it imparts its energy to a 'brake'), but constantly cycles between the two types, the repetition rate at which this happens is usually a natural constant depending on the construction, and is, barring non-linear effects, not dependent on the amplitude. This property is universally applied to make time and frequency references.

A second useful property of resonant systems (stated as a reason in chapter 3 ) relates to its ability to store energy. Applying an effort (force, torque, voltage etc) to such a system in a periodic fashion at appropriate phase continually adds energy to the system, -similar to a series of well timed small pushes causing a large amplitude in a children's swing- and this can be used to achieve amplitudes larger than those possible with the same effort directly (in a non-resonant system). In other words, less effort is needed to achieve the same amplitude.

Consider the following simple mass-spring-damper system:

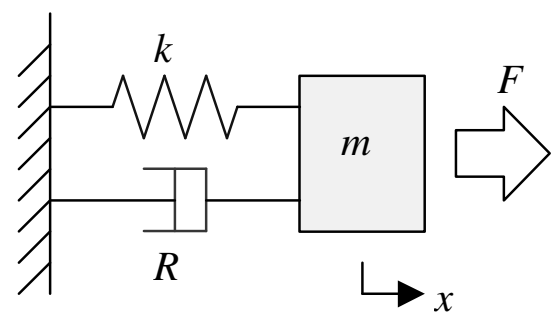

Figure 4.1 Mass-spring-damper: simple ( $2^{\text {nd }}$ order) system

Here, the force $\mathrm{F}$ brings about deflection of the spring ( $F=k \cdot x$ ), the motion of the damper $(F=R \cdot \dot{x})$ and acceleration of the mass $(F=m \cdot \ddot{x})$. If the applied effort (force) is zero, the damping is reasonably small, and the system has some initial energy (e.g. an initial deflected 
position), the mass will continue to describe a so-called "simple harmonic motion". The eigenfrequency (natural frequency, $f_{\text {eig }}$ ) $[\mathrm{Hz}]$ of this motion is given by:

$$
2 \pi \cdot f_{\text {eig }}=\omega_{\text {eig }}=\sqrt{\frac{k}{m}}
$$

Also, given the force-balance

$$
F=k \cdot x+R \cdot \dot{x}+m \cdot \ddot{x}
$$

the system transfer function (Bode plot of deflection-per-applied-force, normalized for unit stiffness) looks like:
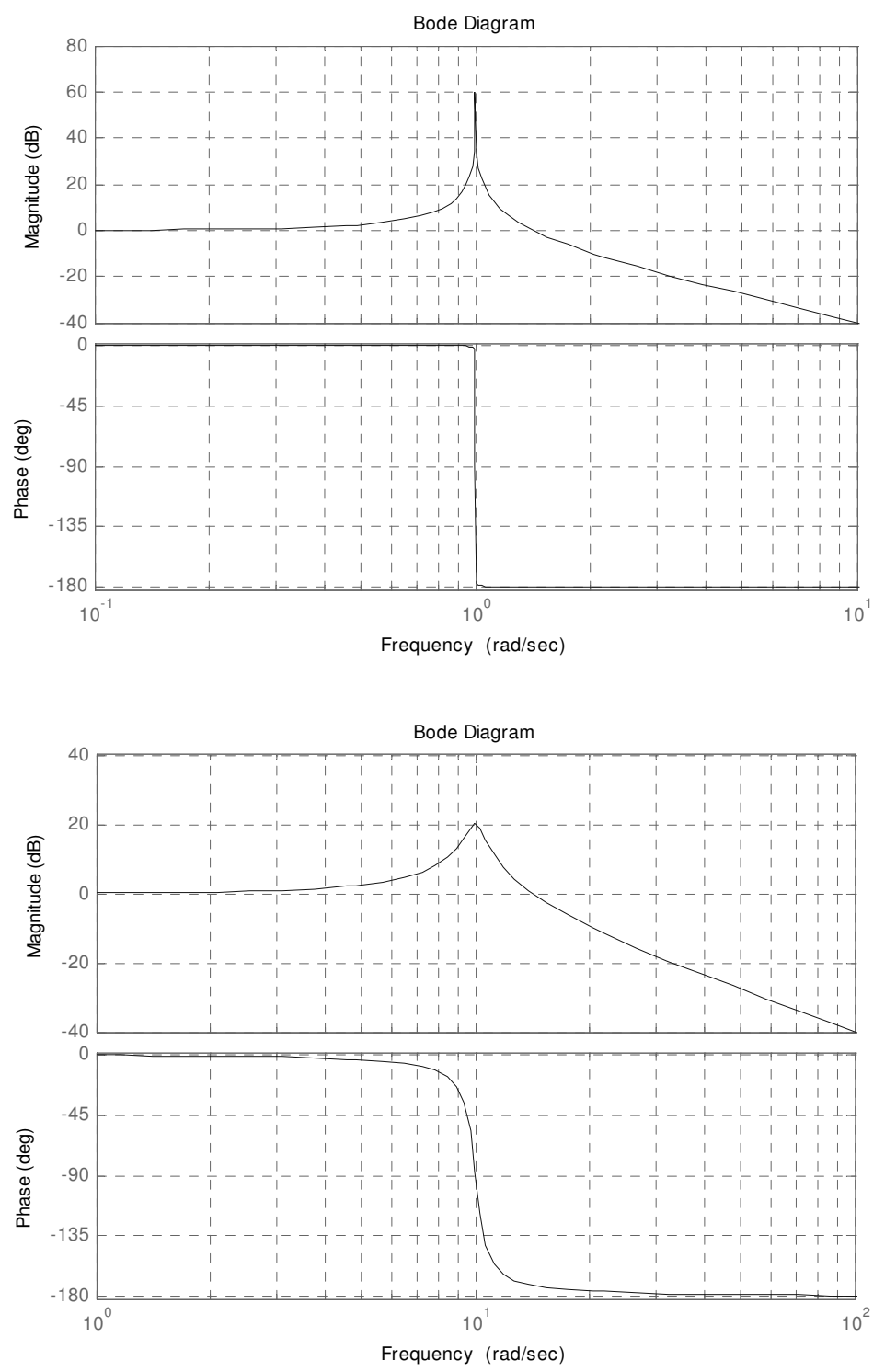

Figure 4.2 Illustrative transfer functions $(X / F)$ of $2^{\text {nd }}$ order systems: $Q=1000$ (top pair) and $Q=10$ (bottom pair)

Notice the sharp phase transition \& narrow peak in the system with the high $Q$-factor 
The magnitude plot can be subdivided into three distinct parts, named "the stiffness line", "the resonance peak" and "the mass line".

The stiffness line is the flat line to the left (lower frequencies) of the peak. Here, the stiffness is dominant, and the deflection $\mathrm{x}$ follows the rule $F=k \cdot x$, or in other words, $\frac{x}{F}=\frac{1}{k}$. Here, $R \cdot \dot{x}$ and $m \cdot \ddot{x}$ are negligibly small as the frequency is low. Furthermore, the phase is zero. This signifies that the deflection directly tracks the force.

To the right of the resonance peak, the mass (inertia) is dominant and the relation between the deflection and the applied force predominantly follows the relation:

$F=m \cdot \ddot{x}$, or in other words, $\frac{x}{F}=\frac{-1}{m \cdot s^{2}}$. Here the magnitude drops as the square of the frequency, inversely proportional to the mass - hence the name "mass-line". The phase is $180^{\circ}$, indicating that the mass moves in an opposite sense than the applied force - logical considering that the force directly causes acceleration, which must be integrated twice in time to get deflection.

Resonance occurs at the eigenfrequency. The magnitude of the resonance peak is determined by the magnitude of the damper, in relation to the mass and spring. It is $Q$ times larger than the magnitude of the stiffness line, where $Q$ is the "quality factor" of the mass spring system; the lower the damping, the higher the 'quality'.

In terms of $m, k$ and $r$, the peak multiplier (quality factor $Q$ ) is given by:

$$
Q=\frac{\sqrt{m \cdot k}}{R}
$$

In other words,

$$
\left[\frac{x}{F}\right]_{\text {peak }}=Q \cdot \frac{1}{k}=\frac{1}{R} \sqrt{\frac{m}{k}}
$$

The term "quality" originates from resonant devices such as tuning forks or oscillator crystals, where the higher the peak, the better the quality of the device (precise frequency; better rejection of nearby frequencies etc.)

It is important that exactly at the eigenfrequency, the phase-shift between the applied force and the resulting (large) deflection is exactly $90^{\circ}$. This means that the velocity $\dot{x}$ is exactly inphase with the force; that the system appears purely resistive to the applied effort. This phase relation can be used to drive a mass-spring system into oscillation; the effort must be inphase with the velocity, or 90' leading the deflection (analogy: push the swing hardest when it is at the null position).

Often, the stiffness $k$ and mass $m$ of a resonant system are easy to determine, by simple calculations or modeling. The (unintentional) damping is often very small and difficult to estimate. However, the Q-factor can be experimentally determined in the following (simple) manner:

- Give an initial energy to a resonator (e.g. - hit the tuning fork)

- Observe the excursion of the system; this will be sinusoidal, with a frequency $f_{\text {eig }}$, beginning with large amplitude, and then will gradually decay, with a decay timeconstant, $\tau_{\text {decay }}$. 


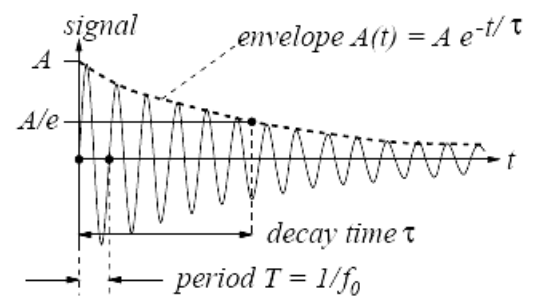

Figure 4.3 Decay envelope after a single hit

- The Q-factor is:

$$
Q=\pi \cdot f_{\text {eig }} \cdot \tau_{\text {decay }}
$$

\subsubsection{Length, diameter and aspect ratio of the Coriolis tube}

From chapter 3 , it is known that the sensing tube is to be made of stainless steel, or a similar material (e.g. titanium). This sensing tube has to withstand a certain pressure [100 bar (i.e. $1 \mathrm{e} 7 \mathrm{~Pa}$ )], and should carry the rated flow at a pressure drop not exceeding [1 bar]. From the previous PoP studies, it is also likely that the Coriolis force will be measured indirectly, employing the deflection of the Coriolis tube; a higher deflection translates to a higher sensitivity (desirable).

- Choosing a tube that is too thick will increase the mass and stiffness, hence reduce the sensitivity

- Too thin a tube will cause excessive pressure drop at rated flow

- Choosing too thick tube walls will increase the mass and stiffness

- Too thin walls might lead to deformation of tube geometry under pressure, changing tube properties significantly

- Too high an aspect ratio (ratio of the length of the tube to the diameter) will result in a low eigenfrequency of oscillation. Gravity will introduce sag in the tube, significantly changing tube geometry. The reduced frequency means lower angular velocity (for the same oscillation amplitude) and hence reduced sensitivity; this is contrary to the fact that increased length increases Coriolis force.

- Too low an aspect ratio will give high eigenfrequency of the tube. If the oscillation frequency is higher than the lowest eigenfrequency of the instrument chassis, there is a risk of exciting chassis eigenmodes, causing non-deterministic behavior

This gives an 'order of magnitude' starting point for the diameter, length and wall thickness of the Coriolis meter tube and is a starting point for tube size selection.

\subsubsection{Shape and form of the sensing tube}

Being able to carry flow, a Coriolis meter tube must have (at least) one inlet and one outlet. The simplest possible tube is a single straight tube of uniform cross-section fixed at both ends. The tube can be further developed into a shape that offers particular advantages. Coriolis tube-shapes from several patents are illustrated in Ch. 2 and Fig. 4.4; many more are to be found elsewhere. 


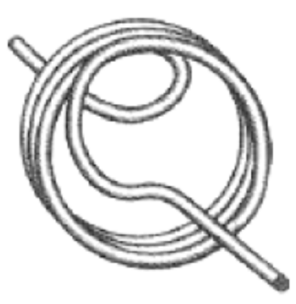

Helix tube Bailey-Fischer \& Porter

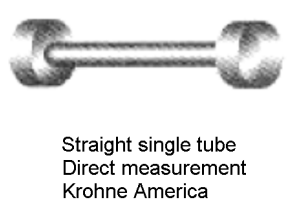

Direct measurement

Krohne America

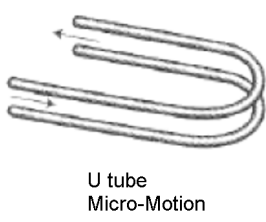
Schlumberger

B tube ABB K-flow
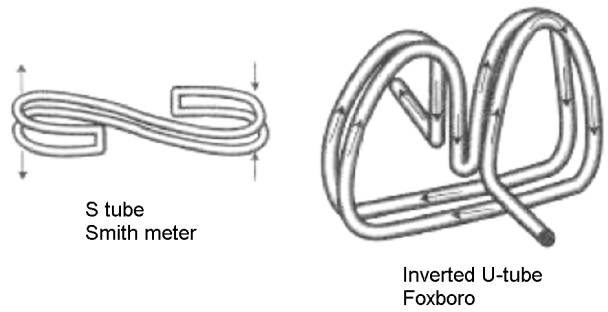

Straight split tube Honeywell

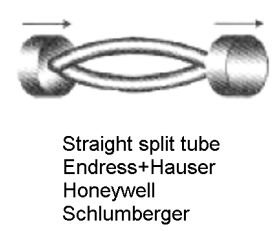

Foxboro
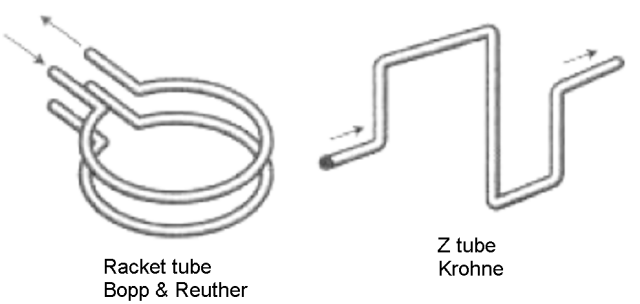

Figure 4.4 Tube shapes in Coriolis meters (from [15])

Due to the multitude and the nature of tube-properties that can be manipulated and goals that can be sought, it is difficult, perhaps impossible, to arrive at "the one best tube shape". We present here arguments that lead to the choice of a particular shape/form, a preview of which has been presented in Ch. 2.6.2.

Fluid connections:

The Coriolis tube has to carry flow - an inlet and an outlet are thus necessary. These have to be attached to the fixed world. The properties of the attachment should not affect tube properties and motion. This suggests the use of a statically determined, vibration-free foundation. If the inlet and outlet of the tube can be placed close to each other, thermal stresses in the foundation are less likely to distort the shape of the tube. The tube has to oscillate in order to generate the Coriolis force - hence space is needed around the tube where it can move. This prohibits the use of intersecting "folded" forms, typically seen in optical systems. The oscillations in the tube may also cause vibrations in the foundation; the foundation should withstand this, and at the same time not affect the oscillation properties.

A shape conducive to distinct and independent modes:

The Coriolis force is generated in a direction perpendicular to the mass flow, and also the rotation. Given that the tube oscillating at an eigenmode causes rotation, the Coriolis force will act on a different eigenmode of the tube. As seen in Ch. 2, the magnitude of this force is very small. In case the force is to be sensed directly, the tube form should be such that the oscillatory motion does not load the dynamic range of the sensor. In case the force is to be sensed indirectly (i.e. by observing deflection in the tube), the tube deflection mode (the response) should have a well-defined characteristic (transfer function gain) at the oscillation frequency. This suggests, that the eigenfrequency of the response-mode should be away from the oscillation frequency. (This is contrary to the intuitive placement near the eigenfrequency 
in order to maximize gain; near the eigenfrequency, the gain and the phase change a lot with minor property-changes). The unused oscillation modes of the tube should be designed far away from the excitation and response modes, to prevent parasitic interactions.

Maximizing the response:

To generate a large Coriolis force, the rotating tube segment should be as large as possible. The tube should be compliant in the response mode (if responding on the stiffness-line) or light (if responding on the mass line). The moment-arm of the Coriolis force upon the response mode should be as large as possible. This increases the achieved mechanical deflection (from a small Coriolis force).

A mechanically "closed" form:

To minimize the effect of unavoidable asymmetries, (see Ch. 3, TR 2.0) the energy storage elements (elastic element, and center of mass) of the oscillatory system should be close to the axis of rotation. For the same reason, it is advantageous to have the possibility to place the oscillation-actuator near the rotation-axis of the response-mode

Based upon these considerations, we arrive at the "window" shape:

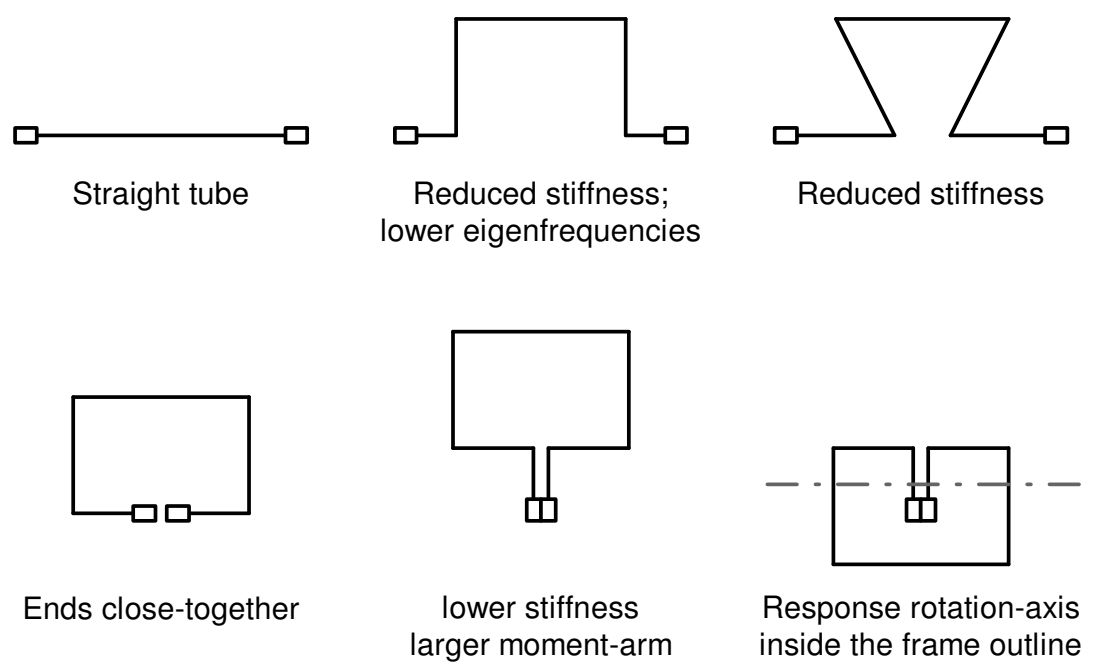

Figure 4.5 Improving the shape of the sensor tube

\subsection{Excitation of the sensing tube - generating rotation}

We have chosen to use an eigenmode of the Coriolis tube (with a well placed eigenfrequency) to generate the rotation leading to Coriolis force. As such, the chosen eigenmode needs to be driven into (controlled) oscillations. The various possibilities are discussed here.

\subsubsection{Actuation}

The meter instrument is to be steered by digital (electrical) processing. There are a limited number of known ways to transform an electrical signal into a mechanical domain. These are based upon electric fields (piezo, capacitive comb-drive etc.) or magnetic fields (Lorentz motor, solenoids etc.). Such actuators may act direct or indirectly upon the meter tube in order to excite it.

\subsubsection{Force excitation vs. Position excitation}

Both the actuation mechanisms described above primarily generate a force. Due to the inclusion of a stiff construction (for example in a piezo actuator) it is possible to get a displacement as an output of an electrical stimulus (instead of a force). What simply happens 
here is that if the material stiffness of the piezo element is $k$, and the force generated by applying an electric field across the piezo element is $\bar{F}$, we see a displacement $\bar{x}$ across the element such that $\bar{x}=\frac{\bar{F}}{k}$. The crucial point here is that the piezo stiffness is much higher than the stiffness of the body (tube) being excited, and hence dominant. A similar explanation also holds true if a (force) actuation principle is used to drive an inertial mass that is several times larger than a secondary body (tube) that is coupled to it. The large mass imposes position excitation upon the tube (see the Rheonik "omega" tube in Appendix D). We choose, if possible, direct (force) actuation for the following reasons:

- A minimum force is required as no extra mass or stiffness is involved. In case of an imperfect construction, less force is present to 'leak' onto the Coriolis mode.

- In absence of extra mass, the effect of the density of fluid medium is clear by means of the eigenfrequency of oscillation of the tube. This aids in the computation of medium density and thereby volume flow-rate.

\subsubsection{Possibility of contact-less excitation}

"It is common to all mass flow meters which work according to the Coriolis principle that the Coriolis forces caused by the mass flow and to be evaluated are very small in relation to the other forces involved in the motion of the conduit. This leads to the fact that the Coriolis forces change the basic motion only slightly. In the case of the known mass flow measurement devices, the basic motion of the conduit is changed by other different influences in addition to the Coriolis forces. For example, these changes appear because of the transducers and oscillation generators attached to the conduit in the case of all known mass flow meters. These additional masses mounted on the conduit, on the one hand, reduce the influence of the Coriolis forces on the motion of the conduit because of their own mass, and, on the other hand, lead to the fact that the conduits are changed mechanically in an insufficiently defined way by the attachment of the masses, for example by soldering or welding. Both influences lead to a reduction of the measurement effect, a lower measurement accuracy, and hard-to-define long-term behavior, and play a role, in particular in the case of mass flow devices of small rated mass flow, i.e., smaller design and thus less conduit mass. In particular, a high mass of conduit including attachments, i.e., of the system capable of oscillation, leads to a lower resonance frequency, equivalent to lower Coriolis forces, and thus a reduced measurement effect. Moreover, in the case of masses symmetrically mounted on the conduit, such as, for example, the transducers in general, very precise mechanical tolerances are to be maintained. This requirement leads to an expensive production process."

- Ronald van der Pol, Meter for flowing media, US Patent 5747 704, May 5, 1998

For several reasons, it is advantageous to be able to actuate the Coriolis meter tube without any attached components. In the event that there are attached components, they should be positioned in such a way that the forces caused by a possibly imperfect construction should be separable (in mechanics, or in the time domain) from generated Coriolis forces.

Bearing this in mind, some concepts were explored before \& during the initial PoP tests: 


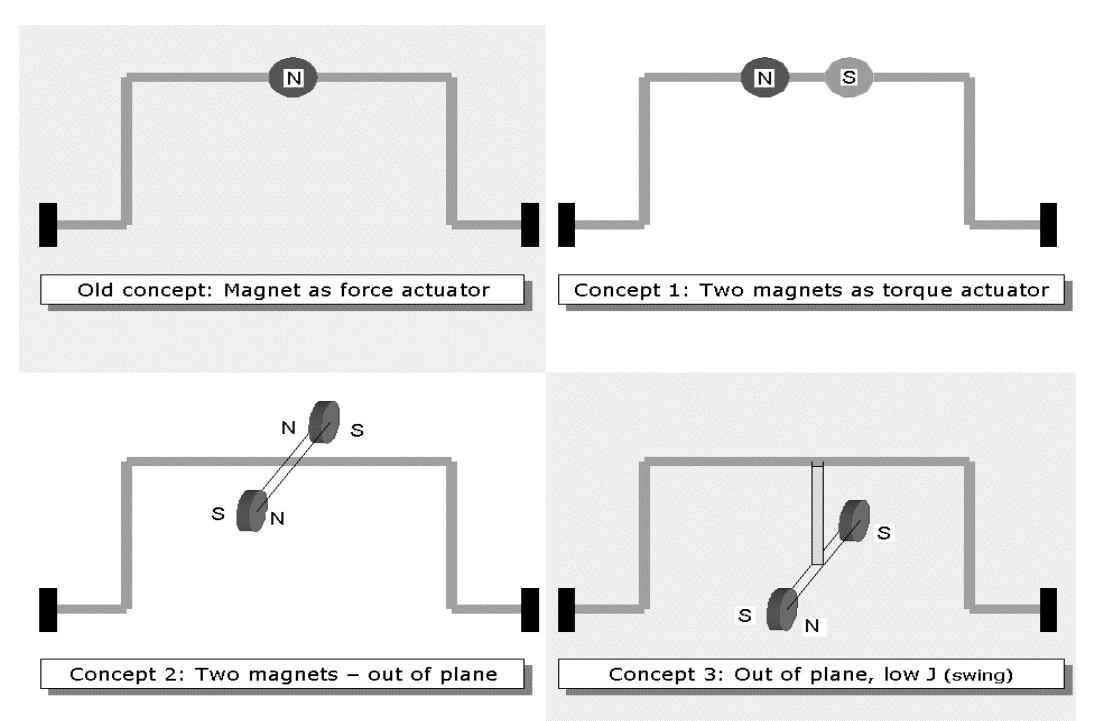

Figure 4.6 Actuator design steps to minimize inter-coupling of actuation and sensing modes

Several optimizations were made to a basic (well known from art) U-shaped tube. An evolution leading to improved meter characteristics is illustrated.

- In step 1, torque actuation is proposed instead of force actuation. If an actuator that generates a pure moment can be constructed, moment being a "free vector", this makes the imbalance-force on the tube independent of a sideways positioning error of the actuator.

- In step 2, the actuation magnets are moved out of the plane of the tube. This reduces the extent to which an imperfect torque actuator excitation would affect the response mode.

- In step 3, the actuation magnet pair is lowered. This prevents the inertia of the magnets from attenuating the Coriolis response (shifts the mass-line upwards). This also moves the actuation plane closer to the rotation axis of the response mode, reducing moment-arm of any "imbalance force". All these and more considerations have been distilled into the prototype instrument.

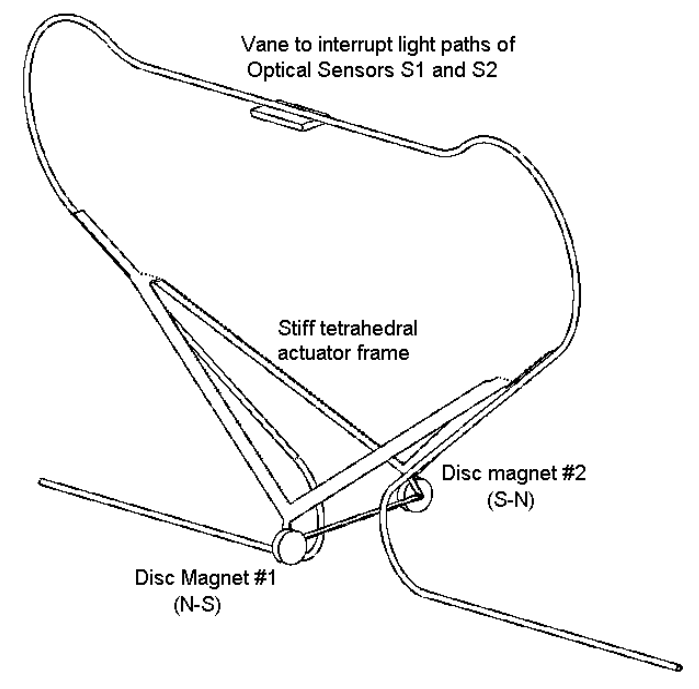

Figure 4.7 Constructional detail of a prototype realization 


\subsubsection{Concept (Part 1) - Lorentz actuator; tube carries current}

The previous construction (Figure 4.7) addresses contactless actuation, but still necessitates a complex construction with a lot of small parts and critical attachments. The (novel) concept of using the tube itself as a current carrying conductor in order to build a Lorentz actuator was explored. Applied to the simple U-shaped tube, this looks like:

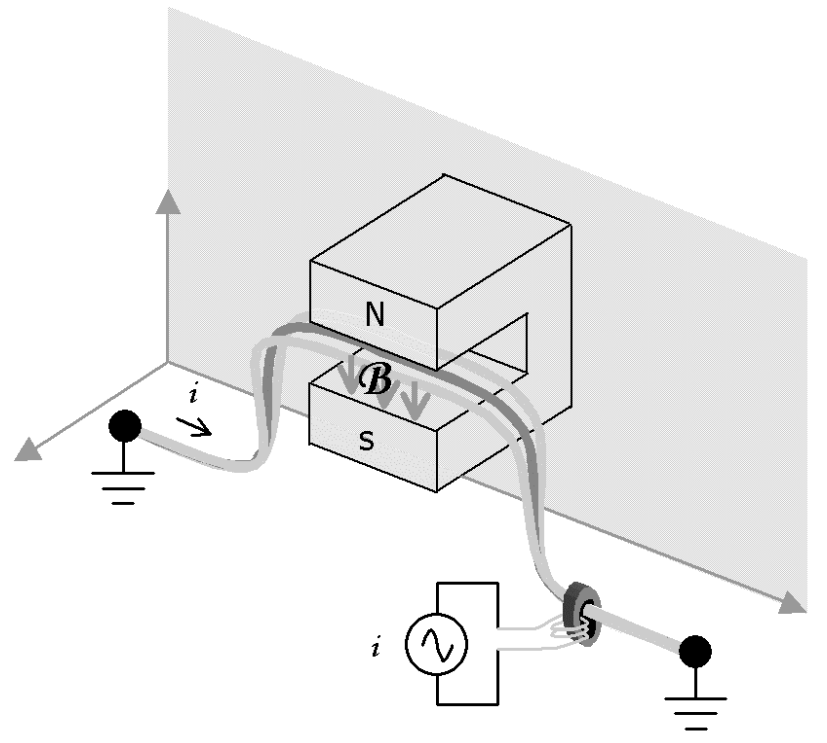

Figure 4.8 A current is induced through the meter tube.

\subsubsection{Concept (Part 2) - Galvanic isolation of excitation current}

In a typical application, it is possible that there exist stray potentials across instruments, leading to currents through them. Such currents, being non-deterministic, would influence forces created in the actuator, at best leading to unwanted actuation, at worst - forces that are indistinguishable from Coriolis forces. It is possible to isolate such currents using an insulating material between the chassis and the (fluid) interface to the external world. The insulating material may cause fluid-compatibility issues - there are only a very few materials that may be allowed to come into contact with the fluid. Furthermore, currents conducted by the fluid itself cannot be avoided in this way. Another way is to bypass most of the external current by providing an alternate path; this would effectively allow only a fraction of the external current to flow through the meter tube. A disadvantage of this second method is that some of the actuation current too is necessarily wasted through this alternate path. If a "mechanically closed loop" concept discussed in Ch. 4.1.3 is used, introducing the current in the tube at all becomes a problem; this would entail the attachment of wires to the (moving part of the) tube. An elegant way to avoid this problem altogether is to use a transformer like construction to introduce a current in the mechanically closed loop - the loop acts as a oneturn secondary winding. A bonus of this method is that we get galvanic isolation between the instrument body and the electronics inside it. This adds to robustness of the electronics, and the electrical safety of the instrument in general.

\subsubsection{Pure torque actuation}

In order to avoid actuator asymmetry influencing the Coriolis mode, a Lorentz actuator principle has been further developed as a "pure torque" actuator. 


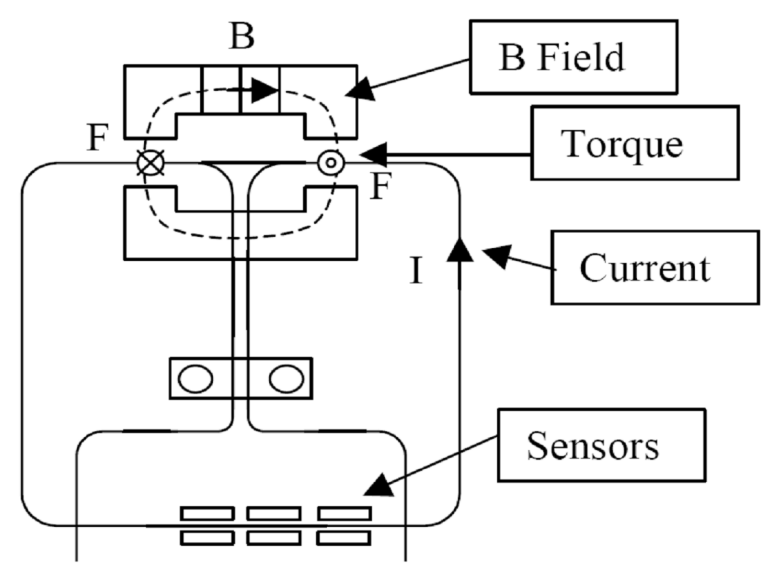

Figure 4.9 Pure torque actuator

The tube is exposed two (oppositely oriented) magnetic gaps (instead of just one), which carry flux lines in anti-parallel directions. As the two gaps are in 'series' it is possible, without the need to match magnets, to get near-identical (opposite) flux densities in the two gaps. As the tube itself is used as the current carries in the Lorentz actuation, the forces generated in the two gaps will be equal-and-opposite - in fact an almost ideal torque. Being a torque actuator, its position (in the horizontal direction in Figure 4.9) does not significantly affect the nature of actuation. Another feature of this actuation method is that it does not conflict with the principle of applying actuation at a point close to the response-mode (swing-mode) rotation axis.

\subsection{Observation of the sensing tube}

So far, we have a tube that periodically rotates (oscillates), and means to introduce a fluidic mass-flow through this tube. The fluid carrying tube has a certain length, determined by its geometry and rotation mode. Depending on the instantaneous angular velocity and mass flow rate in the tube, an instantaneous Coriolis force will be generated in the tube. This force must be determined accurately in order to make an accurate estimate of the mass flow-rate. Forces other than the Coriolis force should not be allowed to this measurement. Typically, such extraneous forces are:

- Windage: Air damping caused upon the (rapidly moving) meter tube causes a couple (and additionally, a force if the damper is asymmetric) upon the tube. Like the Coriolis force, this couple too is proportional to (and hence in-phase with) the instantaneous (angular) velocity of the tube.

- Lorentz force caused by stray magnetic fields

- Forces due to damping in the instrument chassis associated with the vibration of the tube

- External (environmental) vibrations Additionally, the actuation force that sets the tube in motion, if applied incorrectly (due to constructional imperfections) may also be misconstrued as Coriolis force.

\subsubsection{Observing the Coriolis force}

The Coriolis force generated in the sensor tube has a very small magnitude (typically a few micro-Newton at full-scale). This force may be measured in several possible ways:

- Stiff sensor: A force sensor like a Piezo disc is attached to the tube and restricts the motion of the sensor tube, in turn applying a counter force upon the tube. This causes a strain in the force-sensor material, which gives an electrical signal representing the force.

- Compliant spring: The force is allowed to act upon a non-stiff spring, or a small mass, possibly that formed by the sensor tube itself (this means that no additional components and attachments are needed). The force can be inferred from the resulting deflection of the spring (or mass). 
- Stiff controller: A controller and actuator pair (together having a precisely known transfer-function) counteracts the Coriolis force. This "force balance" method minimizes effects of unknown "sensing stiffness" in the two previous methods.

Once again, a concept that affords minimum attachments to the sensor tube is chosen. From PoP studies and a review of various position sensing methods (see [4]), it has been concluded that position (deflection) measurement upon the tube itself by means of optical interruption sensors is suitable:

\begin{tabular}{|l|c|c|}
\hline Method & Quality of measurement & Ease of construction \\
\hline Strain gage & ++ & - \\
Eddy-current & + & ++ \\
Variable reluctance & - & ++ \\
Inductive & + & ++ \\
Magnetic & + & ++ \\
Piezoelectric & ++ & + \\
Capacitive & + & - \\
Interferometer & ++ & - \\
Optical transmissive & ++ & - \\
Optical reflective & ++ & Non critical construction at \\
\hline++ & Accurate measurement - better than & millimeter scale \\
\hline+ & $1 \%$ of full-scale - easily possible & Critical construction \\
\hline- & Marginally suitable & Complex construction, \\
& Insufficient accuracy & bordering on impossible \\
\hline
\end{tabular}

*Adapted from an internal document by Paul P.L.Regtien, August 2002

Furthermore, for reasons discussed in section 4.4, it is essential that the position measurement sensors have as little phase-lag as possible. Optical sensors are well suited to this requirement too.

\subsubsection{Optical position sensing}

The selected optical transmissive (interruption based) position sensing scheme works as follows: An aligned pair consisting of one optical emitter (typically an LED) and one optical detector (typically a phototransistor) forms a basic sensing entity. Such pairs are available for industrial optical interruption detection from manufacturers such as Omron, Vishay, Fairchild, etc. as COTS parts. Most of the light from the emitter traverses a gap and is incident upon the detector. By placing an occluding element in this gap, the amount of light reaching the detector may be modulated.

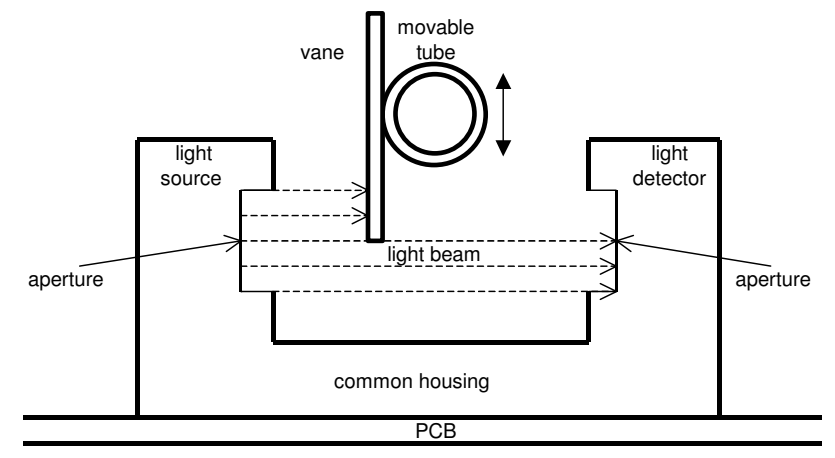

Figure 4.10 Occlusion-based optical position sensing

In the detector, the amount of light reaching it generates a proportional photocurrent. This may be converted to an analog signal suitable to be digitized and interpreted by a DSP. Nominally, the occluding element is adjusted to block about half the light that would reach the 
detector. As the occluding element (in our case, a vane attached to the sensor tube) moves, the modulated light-beam causes an electrical signal proportional to the extent of occlusion. One or more optical interruption sensors may be positioned to sense the movement of the meter tube. The motion resulting from Coriolis force in the active (rotationally actuated) portion of the tube is a periodic translation. The rotation and translation motions, being modally orthogonal, appear as a superposition. These two motions (henceforth called excitation and response) should be separated in order to isolate the response motion (which represents the Coriolis force). Two factors can be used to aid this separation:

1. As the excitation motion is a rotation, it has an axis. At this axis, the position change of the tube due to rotation is zero; the motion here is caused purely due to translation. Alternatively, if two position-sensors observe the tube symmetrically around the rotation axis, the common-mode signal (mean of the two) corresponds to the translation, while the difference corresponds to the excitation (rotation) motion.

2. The Coriolis force is proportional to the instantaneous angular velocity of the tube, which is excited in a simple harmonic (sinusoidal) motion. The position due to the excitation is then 90' phase-shifted from the angular velocity. The deflection due to the Coriolis force is, however, in-step (purely in phase or purely anti-phase) with the angular velocity. Knowing the exact phase of one of the two - the excitation or the response - can enable the isolation of the response motion.

\subsubsection{Electrical representation of optical signals}

For reasons discussed in section 4.4 , it is essential that the position sensor have a small latency (time interval between an event happening and it being read-out). From PoP tests it is evident that this latency (or rather, the variation thereof) should be smaller than 20 ns. In an optical interruption sensor, the main source of latency (and also variation) is the charging/discharging time of parasitic capacitors in the transistor structure. The light incident upon the phototransistor generates a photocurrent. Typical analog-to-digital converters accept a voltage signal. The simplest way to convert the photocurrent to a voltage is to allow it to pass through a so-called "sense resistor". The voltage drop across the sense resistor is proportional to the photocurrent. Herein lies the problem: As the incident light varies, the changing voltage across the resistor must also charge or discharge the parasitic capacitors; the resistance $\mathrm{R}$ of the sense resistor, together with the parasitic capacitor $\mathrm{C}$ forms an RC network. Furthermore, the effective value of $\mathrm{C}$ is a function of temperature, making the delay due to the RC time-constant very temperature dependent (measured variation in equivalent capacitance in PoP tests was about $2 \mathrm{nF}$ - leading to a variation in latency 100x worse than required). Hence, a concept choice is made to use a transimpedance amplifier (see [4] and Appendix C) for converting the photocurrent to a voltage signal.

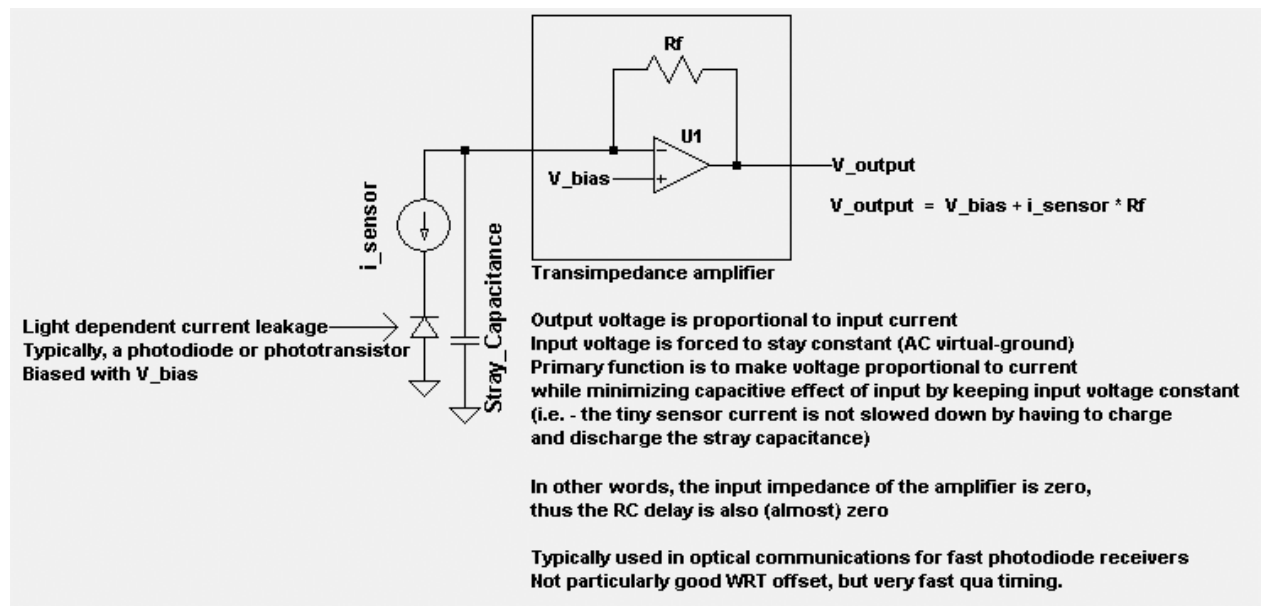

Figure 4.11 Transimpedance amplifier: Operation principle

The transimpedance amplifier (current input; voltage output) intentionally has low input impedance. This means that the voltage variation across the transistor is negligible. As no 
charging and discharging takes place, the parasitic capacitance plays no role. An alternate explanation can be, that as the input impedance of the amplifier is low (zero, ideally) the RC time constant is also is nearly zero; hence no delay.

\subsection{Ratiometric sensing in terms of phasors}

Let us briefly review the chain of events leading to a mass flow measurement: The actuator described in 4.2 drives, with a certain gain, the sensor tube into a rotational mode, with amplitude dependent on the actuation effort and the Q-factor of the mass-spring system formed by the tube. A flow through the tube causes Coriolis force that pushes the tube sideways. A force sensor, with a particular sensitivity, senses this force. This is a measure for the mass flow. Every gain term in this chain contributes to the measured quantity. Each variation in gain is a possible error in measurement. A smart way to avoid dealing with individual component gains is to carry out measurement purely in time/frequency domain. This concept is quite common in phase modulated radio communication systems [16] and has been seen applied to various Coriolis mass flow rate meters in the past decade. The idea is as follows:

1. Two sensors are used to measure the position of the sensor tube. These are placed symmetrically around the rotation axis of the tube.

2. These two position sensors simultaneously measure the superposition of rotation (excitation) of the tube (r. $\boldsymbol{\theta})$ as well as the translation-swing (y) caused by the periodic Coriolis force (each individual sensor cannot tell the two apart, but the two sense a common signal for a translation and a difference signal for a rotation).

3. The excitation motion can be considered a phasor (detailed in Appendix A); the length of the phasor arrow corresponds to the amplitude of excitation as seen by the sensor $(r . \theta)$, and the direction to the phase. As the position sensors are placed on two sides of the rotation axis, the excitation phasors seen by the two sensors are $180^{\circ}$ out-of-phase - thus represented by anti-parallel arrows.

4. The Coriolis force is at its instantaneous maximum when the rotational velocity is highest. At this instant, the rotational position is zero. As the periodicity of the Coriolis force is NOT near the periodicity of the eigenmode of the resulting motion, the 'Coriolis motion' is not a resonance motion - it is either on the mass line or on the stiffness line of the resulting mode. This implies that the 'Coriolis motion' is exactly $90^{\circ}$ out of phase (leading OR lagging) with the excitation motion.

5. Similar to the excitation phasors, the translation response caused by the Coriolis force too can be seen as a secondary (much smaller) phasors, orthogonal to the first, with exactly the same frequency. Being an oscillatory translation ("swing like motion"), this secondary motion corresponds to in-phase (parallel) phasors at the sensor locations. Each sensor, however, sees not the individual (excitation and response) phasors, but rather the sum of the two. (see Appendix A)

6. Under a no-flow condition, two sensors mounted on two sides of the rotation axis would have measured position signals that are, in time, exactly $180^{\circ}$ out of phase. However, in presence of flow, due to the so-called 'Coriolis motion', they register a sum of (anti-phase) excitation phasors and (in phase) response phasors, resulting in an extra phase-shift over and above the $180^{\circ}$ difference.

7. This extra phase-shift is a measure for ratio of amplitudes (response-perexcitation), which in turn is a measure for the Coriolis force. Being a kind of ratiometric measurement, it is dependant neither on the actuator amplification nor the sensor sensitivity nor the rotation amplitude. The other influences are easily characterizable and predictable. The evaluation of the Coriolis force is referenced only to the (in)accuracy of the system clock source.

\subsubsection{Choice of phase detection method}

Various phase detection schemes are available for precisely measuring phase difference between two signals (see [4],[7]). A dual zero-crossing-detector, perhaps the simplest phase detection scheme, is seen in several CMFM patents. As an outcome of PoP studies, we choose for a dual quadrature detection scheme as it offers several advantages: 
- Measurement noise is lower as the detector observes the complete waveform and not just the zero-crossing instants (narrow band measurement - less noise)

- Using a dual quadrature detector (instead of a single phase detector [7],[16]) allows the rejection of harmonics; we can effectively use the knowledge that the motion of the meter-tube is sinusoidal to reject harmonics caused, for example, by the nonlinearity of the position sensors.

- It is feasible to implement dual quadrature detection algorithms on inexpensive commercial DSPs.

\subsubsection{Overview - Dual quadrature demodulation}

A method, very similar to one used in communication receivers [5] and vector voltmeters [16] is briefly described here, to determine the phase difference between the measured position signals from position sensors (say S1 and S2). This detection method (using a PLL algorithm) makes use of the entire waveform to extract phase information. It may be summarized as:

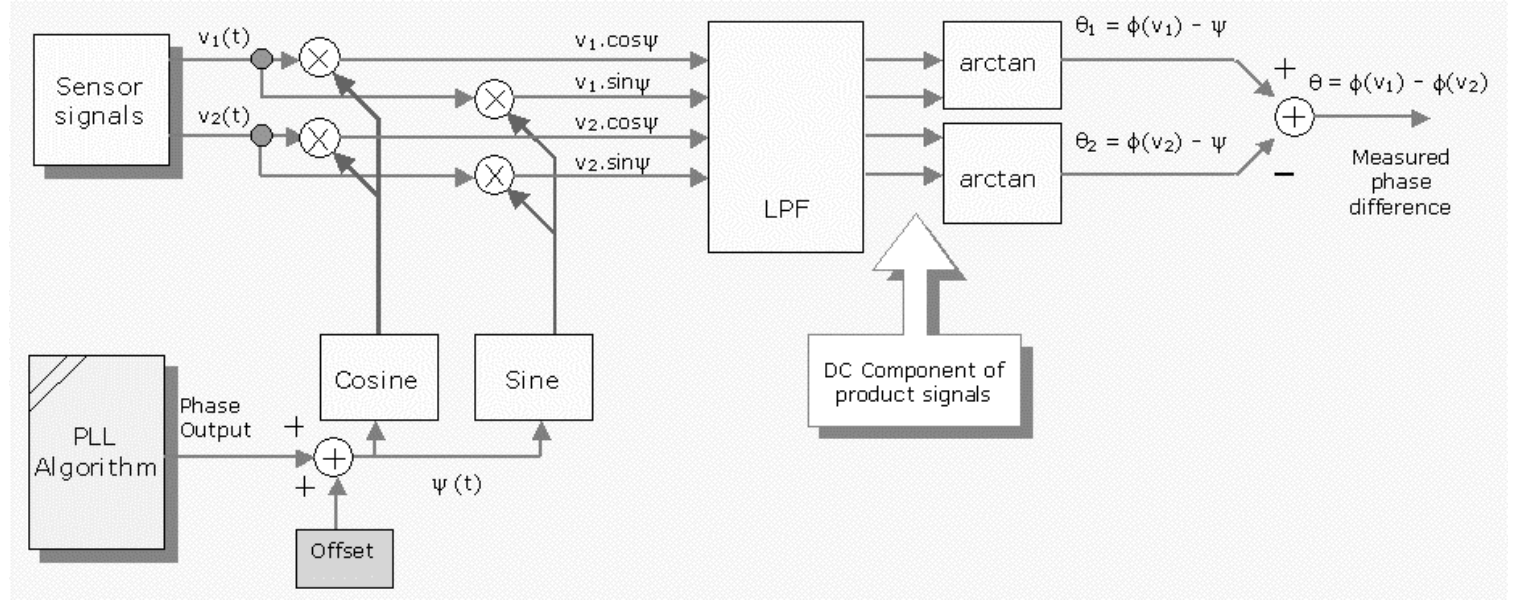

Figure 4.12 Dual quadrature detector implementation to measure phase difference

1. A PLL-like algorithm (implemented in instrument firmware) generates a reference phase signal locked onto the measured phase of the sensor signals. It computes the (eigen)frequency of the oscillating tube referenced to the system clock (crystal oscillator)

2. With prior knowledge about the instrument (oscillator with a high Q-factor) we can guess what the rate of change of the instrument eigenfrequency might be. Based on this rate, we can filter the variable 'computed frequency' (targeted filtering instead of general filtering).

3. Using the filtered frequency and the system time-base, we synthesize two waveforms - a sine and a cosine - in the firmware, having a phase (say $\psi$ ).

4. The two sensor signals are resolved in terms of the reference phase by multiplying them in real time with the reference sine and cosine waves.

5. Multiplication shifts the sensor spectrum by a frequency exactly equal to the instantaneous eigenfrequency. By filtering the result, we can focus on only the primary component of the spectrum, narrowing the bandwidth, thereby improving SNR.

6. The 'resolver' action actually computes the phase of each of the two sensor signals with respect to the reference sine/cosine waveforms $\left(\theta_{n}=\operatorname{PhaseOf}\left(S_{n}\right)-\psi\right)$. As the computation is ratiometric, it does not depend on the amplitude of the measured position signal.

7. For any arbitrary reference phase $\psi$, the difference between the two resolved phases $\left(\theta_{1}-\theta_{2}\right)$ always corresponds to the angle $\pi-2 \theta$ (see Appendix $A$ ) and is a metric for the mass flow rate. 


\section{Noise vs. speed tradeoff}

Any measurement system has an associated measurement noise. The noise has a spectrum; by decreasing measurement bandwidth, it is usually possible to decrease noise power. The use of a dual quadrature detector offers algorithmic flexibility to trade-off speed for noise. Adjusting the filter parameters of the low-pass filter module (after multiplication with the sine and cosine reference signals) is a convenient way to achieve this.

\subsection{Digital representation of electrical signals (and vice versa)}

Having made a concept choice to process signals digitally, it is important to choose analog-todigital conversion means that do not degrade measurement. Let us first consider the digitization of the tube-position (sensor) signals. From PoP tests, it has been demonstrated [9] that a 12-bit representation of each position is sufficient to implement basic functionality. In the interest of reducing quantization noise, it is advantageous to use an A/D converter with a higher resolution. It is important to remember that a high accuracy (of magnitude) is not essential, as the measurement is phase-based. However, the (position) measurement must be deterministic (accurate) in time. Any phase mismatch in the measurement instant of the two position signals will be misinterpreted as a phase shift (caused by a non-existent Coriolis force). Various analog-to-digital converters are commercially available. These include flash converters, successive approximation converters, slope integrating converters, sigma-delta converters etc. (see [4]). One particular type, the "audio CODEC" sigma-delta converter, is particularly suited for this application:

- $20+$ bits of resolution

- $48 \mathrm{k}$ samples-per-second typical conversion rate

- Multiple "parallel sampling" converter channels - This means that the position sensors are sampled simultaneously - there is no significant time-interval between the sampling of one sensor and the second sensor; compensation is not required. (This is a big advantage over multiplexed ADCs)

- Interface is tailored for DSP applications; transferring high precision data at high sample-rates to a DSP is efficiently carried out with minimal software overhead Commercial CODEC ICs have an integrated high-resolution digital-to-analog converter, which can be used for driving the actuator to oscillate the tube; a separate DAC is not required. In order to achieve a high resolution, audio CODEC converters have a built-in digital filter. Using this filter and a technique called oversampling [4] (sampling the analog signal at a rate many times higher than the final conversion rate) together with noise shaping, the $20+$ bits of resolution is achieved. However, this filtering leads to a (deterministic) latency of a few milliseconds. This needs to be accounted for in the system design. Due to the cyclic nature of the tube movement, and the absence of fast control loops requiring low latencies, this limitation of an audio CODEC (sigma delta ADC) is not a hindrance for our system.

\subsection{Summary}

In this chapter, the background and concepts regarding several aspects of Coriolis meters have been discussed. In particular, the discussions concern

- the shape of the tube

- the nature of oscillations

- various actuation concepts, and a particular near-ideal torque actuation concept

- sensing of tube position

- the processing of measurements

In case of multiple concepts, where appropriate, one of the possibilities has been chosen with qualitative arguments. 


\section{Detailed design}

In order to design a mass flow rate measuring instrument as satisfying conditions laid down in chapter 3, we have considered several concepts in chapter 4, weighed them according to merit, and arrived at some concept choices; this entails the use of expert knowledge and experience. Several PoP tests have been carried out at the same time to create insight into the specific problem at hand. Modeling and simulations complement these two activities in order to investigate "what if" scenarios and to generate order-of-magnitudes (numbers) for the chosen concepts; they consider (a simplified version of) the underlying physics. Based upon this physics, a detailed analysis can yield analytical expressions that characterize these nuances of the instrument in addition to its macro behavior.

This chapter deals with the detailed design of the instrument; in this sense it is more precise than the previous chapter - numbers and expressions are provided along with the analysis.

\subsection{The meter tube - Shape and dimensions}

- Instrument has base dimensions of (H)100 mm x (B)60 mm x (D)30 mm; from PoP tests it has been seen that a rectangular closed-frame form of the tube is suitable. It is reasonable to assume that the entire meter tube will have a length that is about 6 times the base of the rectangle, which in turn is about $70 \%$ of the chassis base ( 4 sides of the rectangle +2 middle sections - inlet and outlet)

- It is known that the meter tube should be as light as possible, but at the same time carry, depending on the type, a certain mass flow rate of water, with the resulting pressure drop not exceeding 1 e5 $\mathrm{Pa}$ (1 bar)

- The tube should withstand 1e7 Pa (100 bar) pressure without bursting

\subsubsection{Diameter of meter tube}

Fluid flow through a tube (see [13] pp A4/26 to A4/30) can be approximated as one of two types - laminar or turbulent. The Reynolds' number of a fluid flow is given by:

For a circular tube:

$$
R n=\frac{\rho \cdot v_{\text {mean }} \cdot I D}{\eta}
$$

Where

$\begin{array}{ll}R n & \text { Reynolds number }[-] \\ \rho & \text { Density of fluid }\left[\mathrm{kg} / \mathrm{m}^{3}\right] \\ v_{\text {mean }} & \text { Mean velocity of fluid through tube }[\mathrm{m} / \mathrm{s}] \\ I D & \text { Diameter of tube }[\mathrm{m}] \\ \eta & \text { Dynamic viscosity }[\mathrm{Pa} . \mathrm{s}], \text { in other words, }[\mathrm{kg} /(\mathrm{m} . \mathrm{s})]\end{array}$

Generally, for a flow having Reynolds' number lower than 2300, the flow is laminar, while Reynolds' number higher than 2300 suggests that the flow is turbulent.

Depending on the flow type, for a hydraulically 'smooth' tube, a friction coefficient may be estimated for the fluid:

$$
f_{\text {lam }}=\frac{64}{R n}
$$




$$
f_{\text {tur }}=\frac{0.3164}{\sqrt[4]{R n}}
$$

Where

$f_{\text {lam }} \quad$ Friction coefficient with laminar flow [-] (Laminar: $R n<\sim 2300$ )

$f_{\text {tur }} \quad$ Friction coefficient with turbulent flow [-] (Turbulent: $R n>\sim 2300$ )

Further, depending on the length of the tube in question, the pressure drop across the tube may be estimated:

$$
\Delta P=f_{x x x} \cdot \frac{L}{I D} \cdot \frac{1}{2} \cdot \rho \cdot v_{\text {mean }}^{2}
$$

Where

$\Delta P \quad$ Pressure drop across tube $[\mathrm{Pa}]$ or $[\mathrm{N} / \mathrm{m} 2]$ or $\left[\mathrm{kg} /\left(\mathrm{m} . \mathrm{s}^{2}\right)\right]$

$f_{x x x} \quad f_{\text {lam }}$ or $f_{\text {tur }}$, the friction coefficient [-], to be used appropriately

$L \quad$ Length of tube [m]

ID Inner diameter of tube $[\mathrm{m}]$

It is possible to use these equations, together with the $R n<2300$ condition, to estimate the relation between mass-flow-rate and pressure drop for a given tube diameter. For example, for laminar flows, we have:

$$
\Phi_{v}=\frac{\pi \cdot I D^{4} \cdot \Delta P}{128 \cdot \eta \cdot L}
$$

Where

$\Phi_{v} \quad$ Volume flow-rate $\left[\mathrm{m}^{3} / \mathrm{s}\right]$

Further, it is possible to relate the mass-flow rate $\Phi_{m}$ and the volume flow rate as:

$$
\Phi_{m}=\Phi_{v} \cdot \rho
$$

Using 1e-3 [Pa.s] as the dynamic viscosity of water and $1 \mathrm{e} 3[\mathrm{~kg} / \mathrm{m} 3]$ as the density, a pair of curves (Mass flow rate against pressure drop, laminar and turbulent) can be plotted. The Reynolds number may be used to appropriately choose one of the two curves for a given flow.

\subsubsection{Wall thickness of meter tube}

Stress in thin walled cylindrical pressure vessel:

Consider a length $d x$ of the pressure vessel 
Consider the cylinder cut along its length into two halves

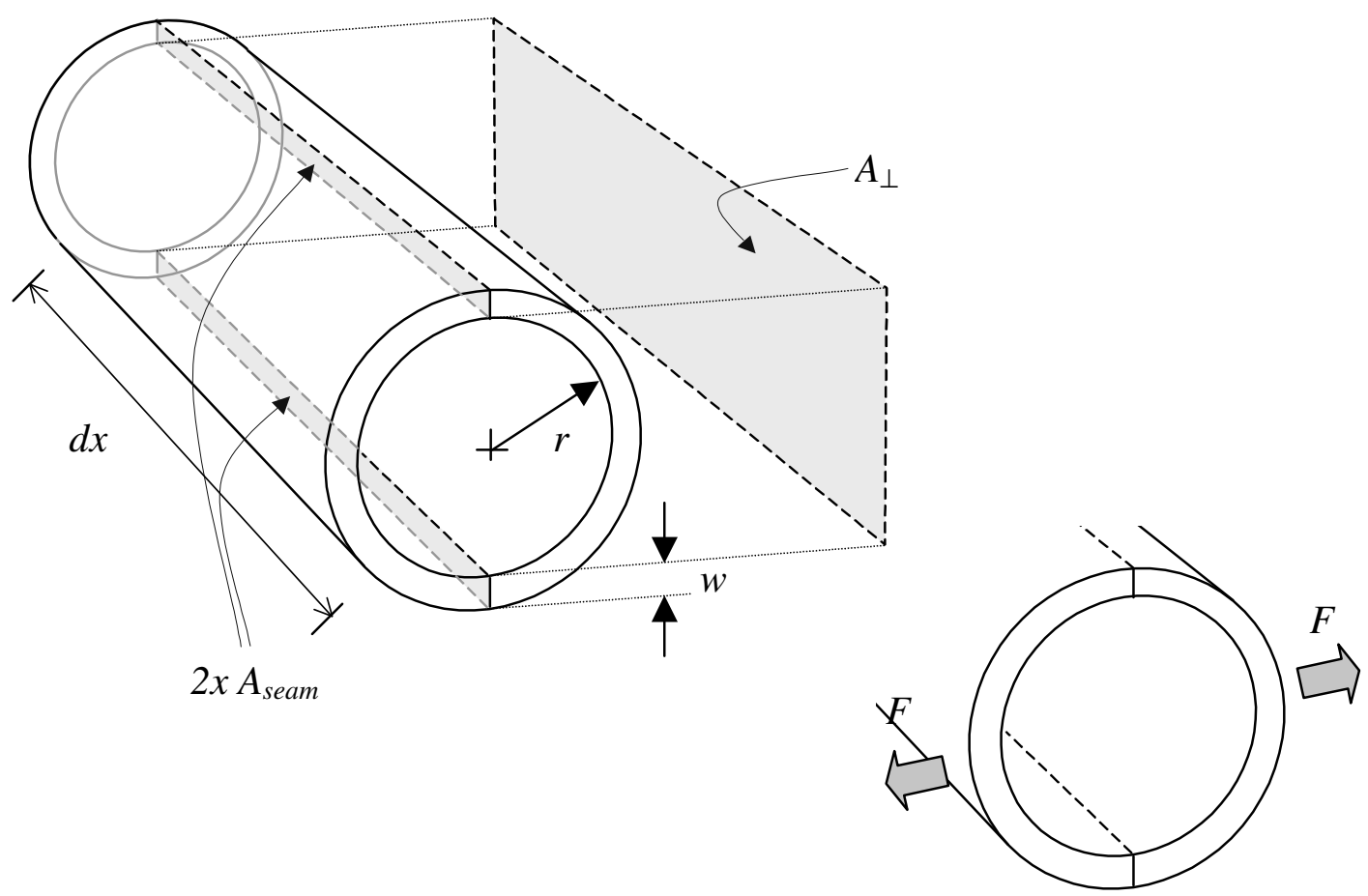

Figure 5.1 Stress in tube wall

Now, the force $F$ on each half (normal surface) is

$$
F=P \cdot A=P \cdot 2 \cdot r \cdot d x
$$

Where

$P \quad$ Pressure in tube [Pa]

$A \quad$ Normal inside-area of a lengthwise-cut cylinder

Now imagine that the cut edges are joined as two seams.

This force is balanced by stress through the two seams

$$
F=2 \cdot \sigma \cdot A_{\text {seam }}=2 \cdot \sigma \cdot w \cdot d x
$$

Equating the two,

$$
P \cdot 2 \cdot r \cdot d x=2 \cdot \sigma \cdot w \cdot d x
$$

Substituting $P=1 \mathrm{e} 7 \mathrm{~Pa}$ (i.e. 100 bar - see $C h .3 .1 .2, F R 14.0$ ), $r=5 \mathrm{e}-4 \mathrm{~m}$ and $w=1 \mathrm{e}-4 \mathrm{~m}$,

$$
\sigma=\frac{P \cdot r}{t}=5 e 7[P a]
$$

This is well below the typical 2 e8 Pa yield strength of AISI 316 stainless steel (see [13], pp E1/49) ${ }^{20}$.

\footnotetext{
${ }^{20}$ And also http://www-ferp.ucsd.edu/LIB/PROPS/PANOS/ss.html
} 


\subsubsection{Shape of meter tube: choosing eigenfrequencies}

The properties of a particular tube-form can be predicted by FEM simulations. The dimension of the tube can be tuned in this manner to follow the conceptual guidelines presented in Ch. 4.1. One consideration is to have the excitation and response frequencies separated from each other, by a factor $>1.5$ - this is to have an acceptably low influence of the system Q-factor upon the response gain. The separation, on the other hand should not be too large $(<1.8)$; a larger separation would mean extra stiffness (or mass) thus lower gain. Another consideration is to place the operational frequencies away from mains frequencies $(50 \mathrm{~Hz}, 60 \mathrm{~Hz})$ and their odd harmonics.

A typical result of an iterative process with FEM simulations is shown in Figure 5.2.
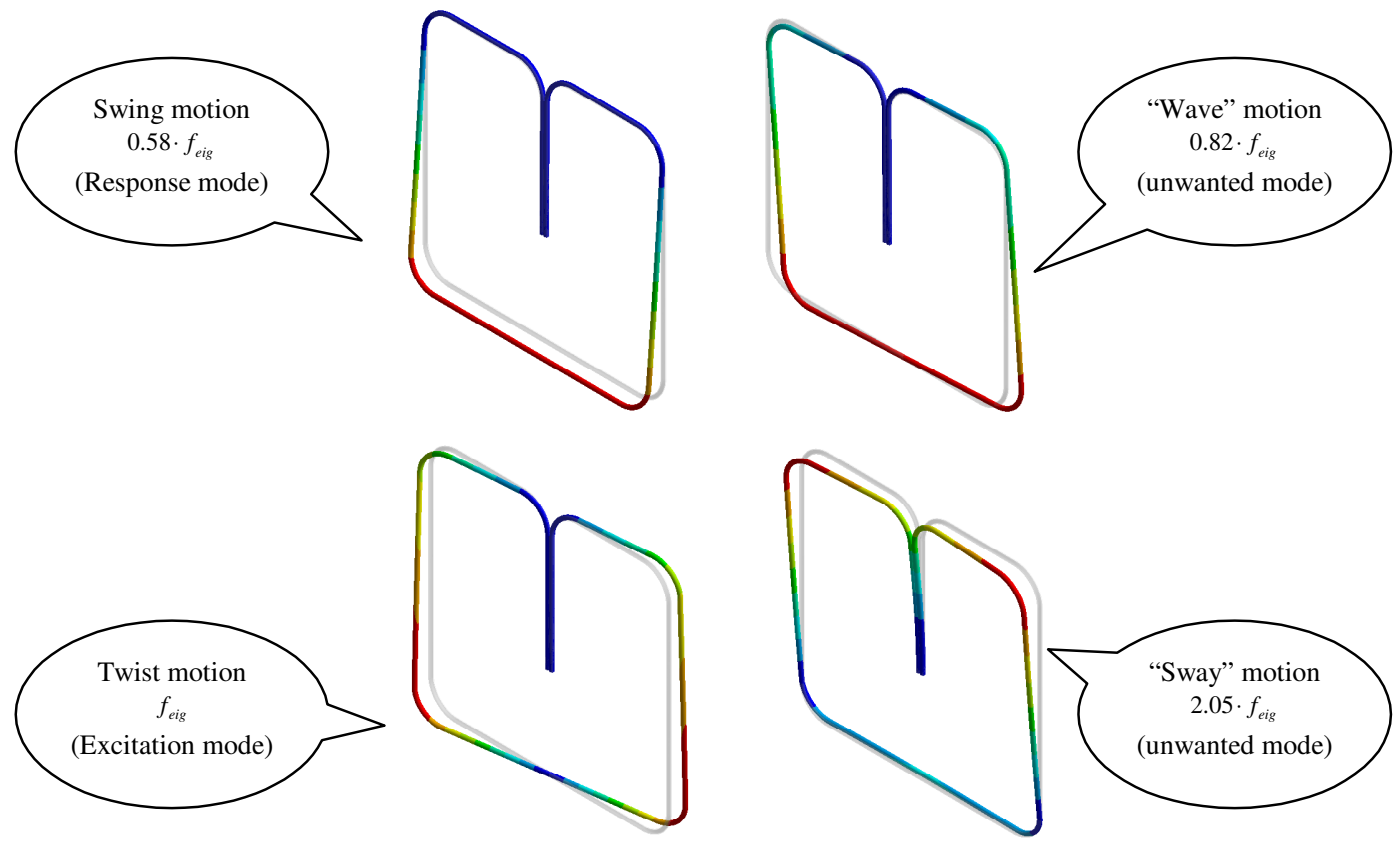

Figure 5.2 Various eigenmodes with a "window" shape

Generally, the swing eigenfrequency is primarily influenced by the height of the 'window' while the twist eigenfrequency is dominated by the width of the window. Both frequencies are affected by the central 'stem' (combined inlet and outlet tube), but the stem-height may be used to adjust the rotation-axis of the swing motion.

For example, due to the folded shape, the rotation-axis of the swing motion lies close to the top-segments of the 'window' shape. This can be used advantageously by actuating the twist motion on these segments. Actuation asymmetry present here will have a small moment-arm on the swing-mode, and will not generate large response-motion.

\subsubsection{Conclusion}

- We have chosen, after careful design of eigenfrequencies, a tube shape that is manufacturable, and separates the function of spring from that of the sensing tube.

- The disadvantages associated with two necessary tube-connections to the 'fixed world' (namely the inlet and the outlet) are overcome by having a single mechanical fixation for both.

- $\quad$ The designed significant eigenfrequencies are well separated from each other and are both at least $10 \mathrm{~Hz}$ away from odd harmonics of $50 \mathrm{~Hz}$ and $60 \mathrm{~Hz}$ (mains frequencies). 


\subsection{Excitation - The oscillating tube}

As discussed in Ch. 2.3 and section 4.2, the Coriolis tube needs to be oscillated. A torqueactuator applies effort at the appropriate period and phase with the right magnitude to put energy into the chosen eigenmode of the tube and thereafter continues to provide just enough power to counteract dissipation. There are several properties of the construction elements that come into play while designing an oscillating tube. These are:

\subsubsection{Q-factor}

The background and measurement procedure for the Q-factor has been discussed in Ch. 4.1. The measured Q-factor can be used for the following:

- Determination of the effort required in the actuator

- Determination of the dissipation in the oscillating tube

- Dissipation generates forces that are in-phase with the velocity of the sensortube, similar to (and if asymmetric, indistinguishable from) the Coriolis force. A larger Q means less dissipation; this makes constructional asymmetries less relevant

- Dissipation can lead to local heating of the construction changing tube properties

- The Q-factor can be used as a diagnostic metric to verify that a sensor tube has been properly constructed. A low Q-factor is indicative of constructional defects.

In PoP tests several tube shapes, construction methods and environmental situations have been tried out. The main causes of a Q-factor that is not infinitely large are:

1. Material-damping in the attachment interface (glue, solder, weld, etc. that attaches the tube to the 'fixed world'), and other construction elements that experience a strain

2. Material damping in the construction material stainless steel of the sensor tube itself

3. Wind damping due to the tube moving rapidly in (viscous) air.

Glue interface

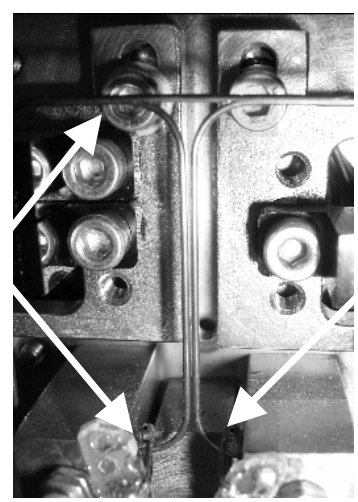

Solder interface

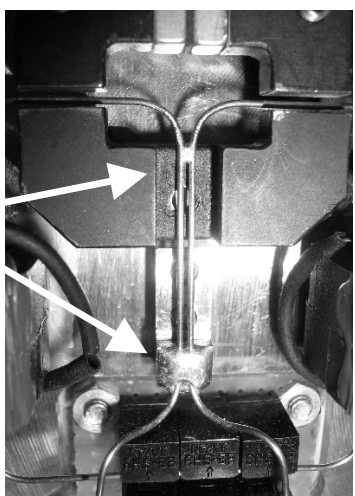

Figure 5.3 Q-factors in various implementations

The results from the Q-factor measurements can be summarized as:

- A compliant attachment material (glue) severely deteriorates the Q-factor

- By using welded or soldered interfaces, a Q-factor of about 2000 can be realized

- In this situation, the air viscosity is responsible for about half of the damping; if evacuated, the system has a Q-factor of about 4500.

- It can be reasoned that the remaining damping is caused by losses in the tube material, and dissipation in 'instrument-to-fixed-world' interfaces 


\subsubsection{Actuation of the Coriolis tube}

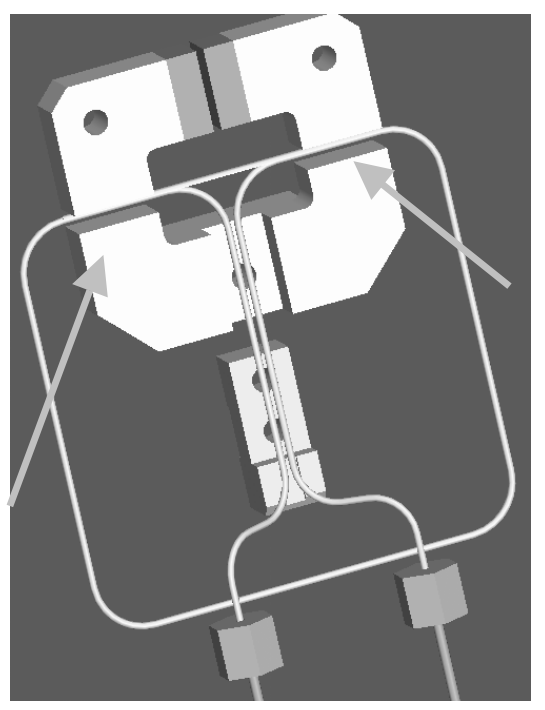

\section{Innovation: Pure torque generator:}

The essential portion of the "Lorentz actuator" concept chosen for driving the Coriolis tube into oscillations is shown here (see Appendix B.1 for details), and works as follows: A current $i$ is introduced in the fluid carrying tube. This current traverses the tube, passing through gaps in a stator magnet yoke. A magnetic field is present in its gap, having a flux density $B$ perpendicular to the tube (and also the current). A force is created in the current carrying conductor, proportional to the cross product of the current, the flux density, and the length of tube $l$ exposed to the magnetic flux.

$\bar{F}=l \cdot \bar{B} \times \bar{i}$

The construction shown here has the following properties:

1) There are two identical, antiparallel air-gaps.

2) The current flowing through the left gap is exactly equal to that through the right gap.

3) Neglecting construction tolerances, this creates a pure couple (effort) upon the tube.

4) This couple twists the central "torsion spring" (the inlet and outlet tube pair), depending on the instantaneous direction of the current

Motor constant of the actuator:

Consider half of the actuator;

\begin{tabular}{|c|c|c|c|}
\hline Moment arm & $r$ & {$[\mathrm{~m}]$} & $\begin{array}{l}\text { Distance between the torsion axis and the centroid of } \\
\text { force acting on half the tube }\end{array}$ \\
\hline $\begin{array}{l}\text { Flux density in air-gap, } \\
\text { upon the tube }\end{array}$ & $\bar{B}$ & {$[\mathrm{~T}]$} & $\begin{array}{l}\mathrm{NdFeB} \text { magnets with a } \mathrm{Br}=1.3 \mathrm{~T} \text { are used to bias the air- } \\
\text { gap; approx. value from simulations }\end{array}$ \\
\hline Current through tube $i$ & $i$ & {$[\mathrm{~A}]$} & AC RMS \\
\hline Effective length & $l$ & {$[\mathrm{~m}]$} & The pole-length over which the Lorentz force is generated \\
\hline Lorentz Force: & & \multirow{2}{*}{$F=$} & $(5.11)$ \\
\hline Torque: & & & $(5.12)$ \\
\hline Motor constant: & \multirow{2}{*}{\multicolumn{2}{|c|}{$k_{m}=\frac{1}{i}=2 l \cdot B \cdot r$}} & $=7.5 e-5[\mathrm{~N} \cdot \mathrm{m} / \mathrm{A}]$ \\
\hline $\begin{array}{l}\text { Torsion stiffness of the } \\
\text { tube construction }\end{array}$ & & & $\begin{array}{l}\text { Depends on material properties and dimensions of the } \\
\text { construction }\end{array}$ \\
\hline Required deflection & $2.8 \mathrm{e}-2$ & [rad] & The amplitude of angular deflection desired \\
\hline Required static torque & $2.0 \mathrm{e}-3$ & {$[\mathrm{~N} . \mathrm{m}]$} & The static torque needed to deflect the tube \\
\hline Typical Q-factor & 2000 & {$[-]$} & Measured - a property of the tube and its surroundings \\
\hline $\begin{array}{l}\text { Required torque } \\
\text { amplitude }\end{array}$ & $1 e-6$ & {$[\mathrm{~N} . \mathrm{m}]$} & Q times smaller than the static torque \\
\hline $\begin{array}{l}\text { Required actuation } \\
\text { current }\end{array}$ & $13.2 \mathrm{e}-3$ & {$[\mathrm{~A}]$} & $\begin{array}{l}\text { The current } i=\mathrm{T} / k_{m} \text { is needed (when the tube is } \\
\text { stably oscillating) to sustain oscillations at the said } \\
\text { amplitude (i.e. to counteract dissipation) }\end{array}$ \\
\hline
\end{tabular}

The "required actuation current" value is for the situation when the sensor tube is stably oscillating; at the time of startup, more current is introduced into the tube to rapidly reach the desired oscillation amplitude. 
The magnitude of excitation current is small enough to be achievable by means of a simple transformer construction, and also does not lead to excessive (heat) dissipation in the sensor tube. The dimensioning of the transformer is discussed in Appendix B.2.

\begin{tabular}{|l|l|l|l|}
\hline Tube loop resistance & 0.6 & {$[\mathrm{ohm}]$} & $316 \mathrm{~L}$ stainless steel tube \\
\hline Required current (RMS) & $1 \mathrm{e}-2$ & {$[\mathrm{~A}]$} & Approx. RMS current required for sustaining oscillations \\
\hline $\begin{array}{l}i^{2} \cdot R \text { dissipation } \\
\text { (heating) in tube }\end{array}$ & $4.8 \mathrm{e}-5$ & {$[\mathrm{~W}]$} & The (negligible) ohmic dissipation in the meter tube \\
\hline
\end{tabular}

The proposed construction seems feasible for a Coriolis sensor-tube oscillator.

\subsubsection{Tolerances essential for the actuator}

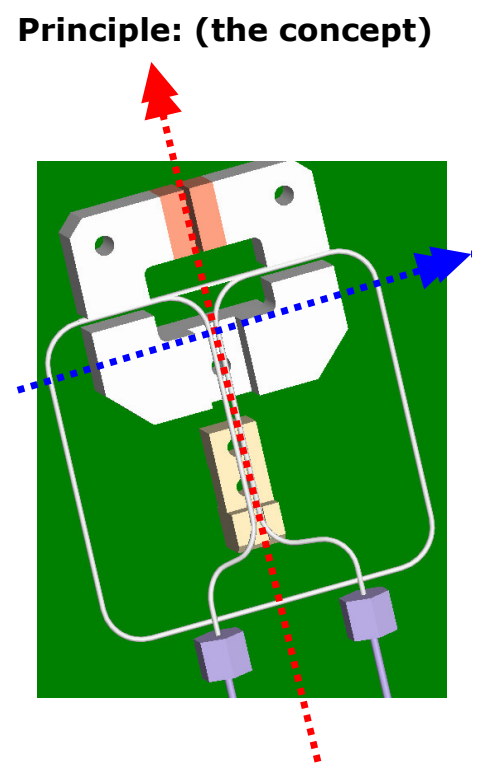

- The actuator drives the tube at its eigenfrequency, with a torque around the vertical axis, a 'twist'. This means that the effort (torque) produced by the actuator is inphase with the instantaneous (angular) velocity of twisting of the tube.

- The Coriolis force(s) too is (are) in phase with the instantaneous velocity, but push the tube around a horizontal 'swing' axis (blue line) and are very small in magnitude. This leads to a situation whereby if a small portion of the actuation effort were to be not a "pure torque" but rather a "torque plus force", this would be indistinguishable from the Coriolis force itself, and would be seen as an offset on the Coriolis force measurement.

- The actuator is designed so as to have an imbalance force that is much less than typically expected Coriolis force; furthermore, the points on the tube where the pair of forces are generated are deliberately near the swing (horizontal) axis, making the moment-arm of any imbalance-force as small as possible.

\section{Possible problems:}

In the concept choice, we have selected an actuator principle that is well suited to generating a pure-torque. However, imperfections in the yoke construction can lead to unequal flux density and unequal active-lengths in the left and right air-gaps. This would lead to the left and right forces to be not exactly equal (and anti-parallel).

The second possible cause of mismatch is unequal electrical current (in the left and right gaps). The mismatch generated in this manner is negligibly small, as the tube is one physical loop, and there are no alternate paths available for the current.

\section{Problem analysis:}

Discussion: constructional imperfections in the magnet-yoke that can lead to unequal flux densities in the left and right air-gaps

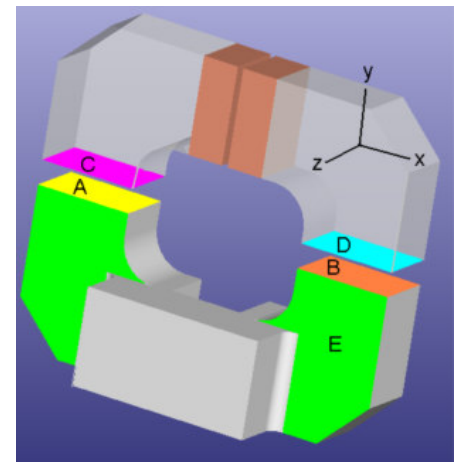

The stator magnet yoke from the actuator is shown here in detail. The pole-surfaces A/C and B/D define the two (left and right) air-gaps. The magnets (in brick-red) cause flux lines through the two air-gaps; as the two gaps are in series, the net flux through the two is the same. However, it is not just the flux density but also the active length that determines the actuation force. This leads to the following:

1) If the width of a pole-surface (in direction of the current) is longer, the area becomes larger; hence the flux density reduces as the width. However, the activelength increases with the width, the net effect being nil.

2) If the distance between the pole surfaces defining one of the gaps is changed, the net flux changes, but is still 
equal in both gaps (as they are in series), causing a different torque, but not an imbalance (force).

3) If the height of one of the pole-surfaces is changed, this leads to a large imbalance. Indeed, the flux in the two gaps is equal, as is the effective length. However, the flux density is less at the pole where the height is more; this causes a mismatch in the force couple (torque)

4) On similar lines, it can be reasoned that surfaces $A / C$ and $B / D$ should be parallel around the $r_{x}$ axis.

Next, let us estimate the extent of allowable mismatch in the actuator construction, using as an example the type (3) situation where the flux in the left and right gaps is unequal due to unequal heights of the pole surfaces.

As stated earlier (see Figure 5.2), the moment arm of an imbalance force is, by design, smaller than that of Coriolis force(s). From geometry, we can say that the Coriolis forces (generated in the rotating top and bottom sections of the tube) have an arm that is about 10x larger than any imbalance force generated at the yoke.

\begin{tabular}{|l|l|l|l|}
\hline $\begin{array}{l}\text { Tube rotation-deflection } \\
\text { amplitude }\end{array}$ & $2.8 \mathrm{e}-2$ & {$[\mathrm{rad}]$} & $\begin{array}{l}\text { Amplitude of the movement that leads to the generation of } \\
\text { a Coriolis force }\end{array}$ \\
\hline $\begin{array}{l}\text { Twist-eigenfrequency of } \\
\text { tube }\end{array}$ & $f_{\text {eig }}$ & {$[\mathrm{Hz}]$} & $\begin{array}{l}\text { The twist amplitude and the twist eigenfrequency together } \\
\text { lead to a rotational angular velocity; the tube describes a } \\
\text { periodic rotation }\end{array}$ \\
\hline Peak angular velocity & 28 & {$[\mathrm{rad} / \mathrm{s}]$} & \\
\hline $\begin{array}{l}\text { Peak Coriolis force at } \\
\text { typ. max. flow }\end{array}$ & $6.2 \mathrm{e}-5$ & {$[\mathrm{~N}]$} & $\begin{array}{l}\text { An antiparallel pair originates in the top and bottom } \\
\text { sections of the tube, causing the tube to 'swing' }\end{array}$ \\
\hline
\end{tabular}

The Coriolis forces lead to a swing deflection; In order to have a small offset, the moment caused by actuator mismatch force should be smaller than a fraction (say 1:1000) of the Coriolis force.

\begin{tabular}{|l|l|l|l|}
\hline Typ. peak tube Current & $1.3 e-2$ & {$[\mathrm{~A}]$} & (during sustained stable oscillation of the tube) \\
\hline Allowed mismatch force & $6.2 \mathrm{e}-7$ & {$[\mathrm{~N}]$} & $\begin{array}{l}1000 x \text { smaller allowed magnitude of moment but 10x } \\
\text { smaller moment-arm, leading to 100x smaller net force }\end{array}$ \\
\hline Length of stator pole & $8 \mathrm{e}-3$ & {$[\mathrm{~m}]$} & From actuator geometry \\
\hline
\end{tabular}

The maximum allowed mismatch in the flux densities in air gaps follows from the relation:

$$
F_{\text {mismatch }}=B_{\text {mismatch }} \cdot i_{\text {actuator }} \cdot l
$$

To have a mismatch-force smaller than $6.2 \mathrm{e}-7[\mathrm{~N}]$, the mismatch flux density must be lower than $5.9 \mathrm{e}-3[\mathrm{~T}]$. Given that the nominal flux density in the gaps is $0.4[\mathrm{~T}]$, the heights of the left and right air-gaps may differ by no more than $1.4 \%$ of their nominal value, i.e. by no more than $35 \mu \mathrm{m}$. This is feasible using standard milling techniques.

A similar analysis may be done for parallel-ness or surface unevenness of the poles: Assuming a near-worst case scenario, where one pole is concave, while the other is convex, leads to a mismatch; this mismatch is acceptably low $(<1.4 \%)$ if the peak-to-peak surface variation is less than about $50 \mu \mathrm{m}$. 


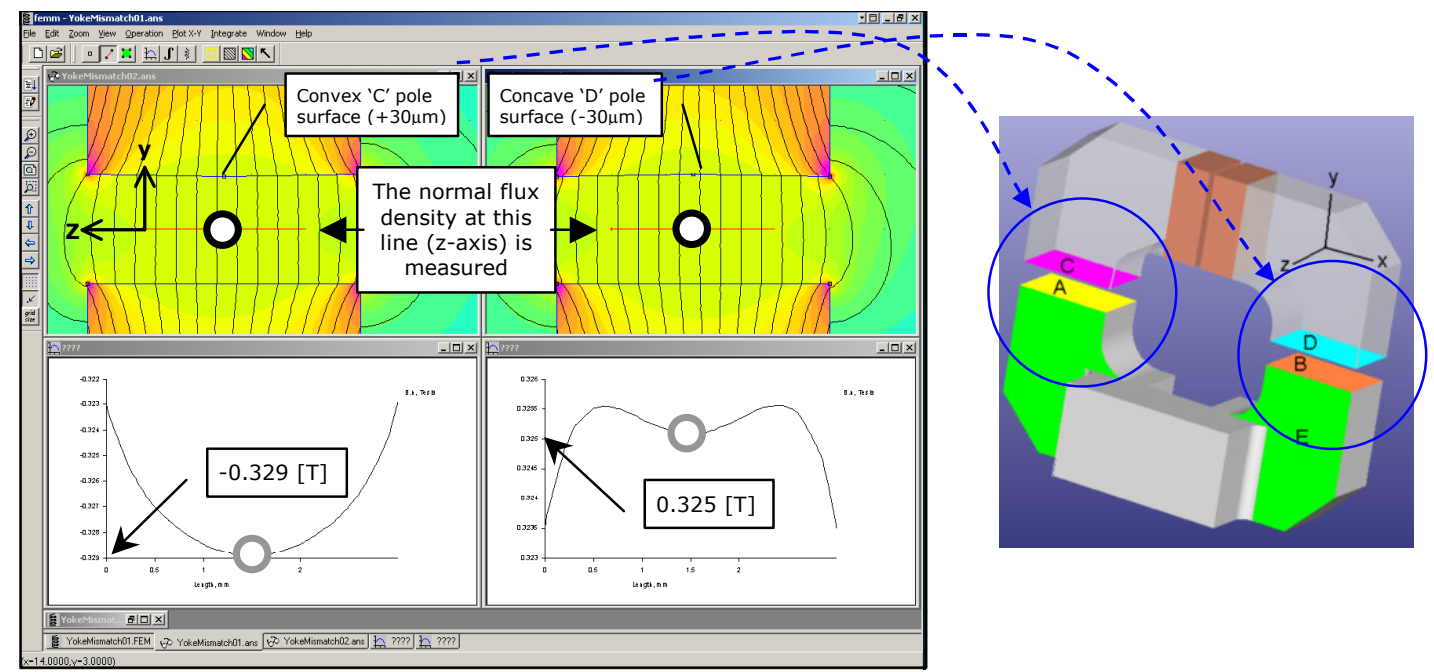

Figure 5.4 The effect of non-parallel pole-shoes on the normal flux density at tube positions along the $z$-axis

\section{Conclusion:}

In conclusion, the design of the actuator is critical, but achievable. Attention is needed to symmetric magnetic design.

\subsubsection{Temperature dependent variation of (stainless steel) material properties}

We excite the Coriolis sensor-tube at its "twist mode" eigenfrequency. The eigenfrequency varies as the square-root of the stiffness of the effective spring and inversely as the squareroot of the effective mass. As the eigenfrequency changes, so does the rotational angular velocity of the tube $\{1\}$. This affects the Coriolis force and introduces a predictable variation of the Coriolis force for a given mass-flow. By means of the eigenfrequency, the instrument is also expected to measure fluid density. However, the tube temperature affects the stiffness, thereby also changing the eigenfrequency $\{2\}$. Both these effects $(1,2)$ have to be corrected for (in order to accurately estimate flow and density).

Accurate temperature measurement is easily possible by means of sensors affixed directly to the tube. Accurate tube-eigenfrequency measurement is possible by means of a crystal clock reference. If we were to know the relation between temperature and the effective stiffness, we could correct for the various physical effects in the following manner: 


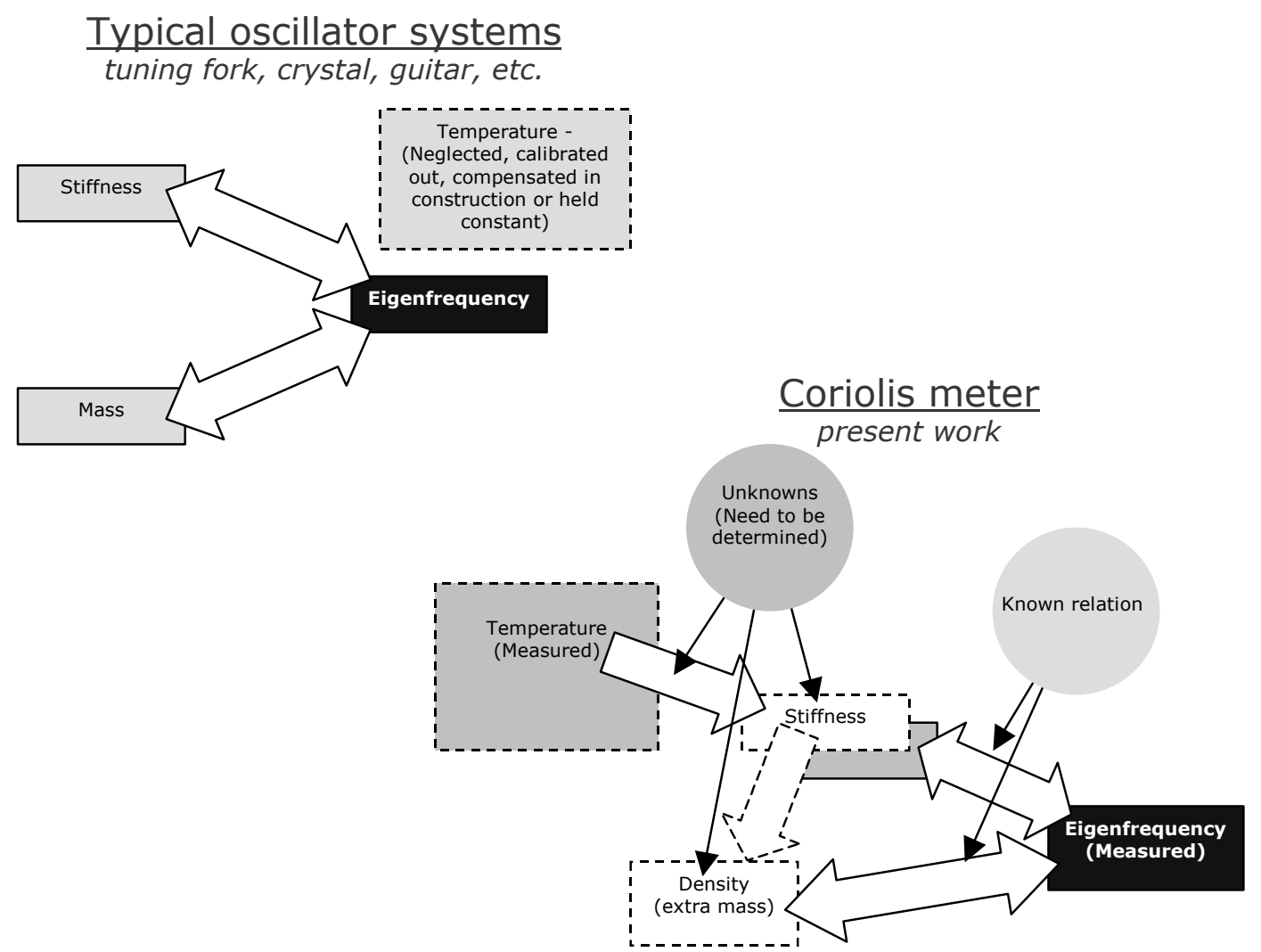

Figure 5.5 Relating temperature to stiffness and stiffness and density to eigenfrequency

Reasoning:

- The Coriolis meter tube is an oscillating system, we excite this in the "twist" mode

- The twist-eigenfrequency is determined by the torsion stiffness and the moment of inertia of the tube

- The tube is filled with a medium, the mass flow rate of which is of interest. The medium adds to the inertia of the tube, reducing the eigenfrequency.

- The medium and the surroundings impart a temperature to the tube

- The temperature of the tube affects two aspects:

- The elastic modulus (stiffness) of the tube material changes with temperature

- The length and volume of the tube also change due to thermal expansion, affecting the amount of fluid in the tube, in turn affecting the moment of inertia, and also the stiffness

- The temperature of the medium affects its density, thereby changing the inertia.

- Beyond these causes, there are no other significant factors (e.g. fluid-pressure in the medium) that influence the eigenfrequency (has been verified experimentally).

If we can determine a sufficiently accurate relation between the tube temperature and two tube-properties - (1) the elastic modulus and (2) the thermal expansion, then by measuring the tube-temperature, we can accurately determine the density of the fluid medium. Furthermore, having separated the inertial attributes and the stiffness attributes, we can predictably relate the minor variation in sensitivity of the instrument to the variation of inertia and stiffness. This relation is determined in 5.3.2. For now, we discuss the effect of temperature on tube-stiffness.

In view of the limited specific information reported in literature regarding this issue, we carried out experiments with sample material (SS - AISI 316 tube) as follows: 


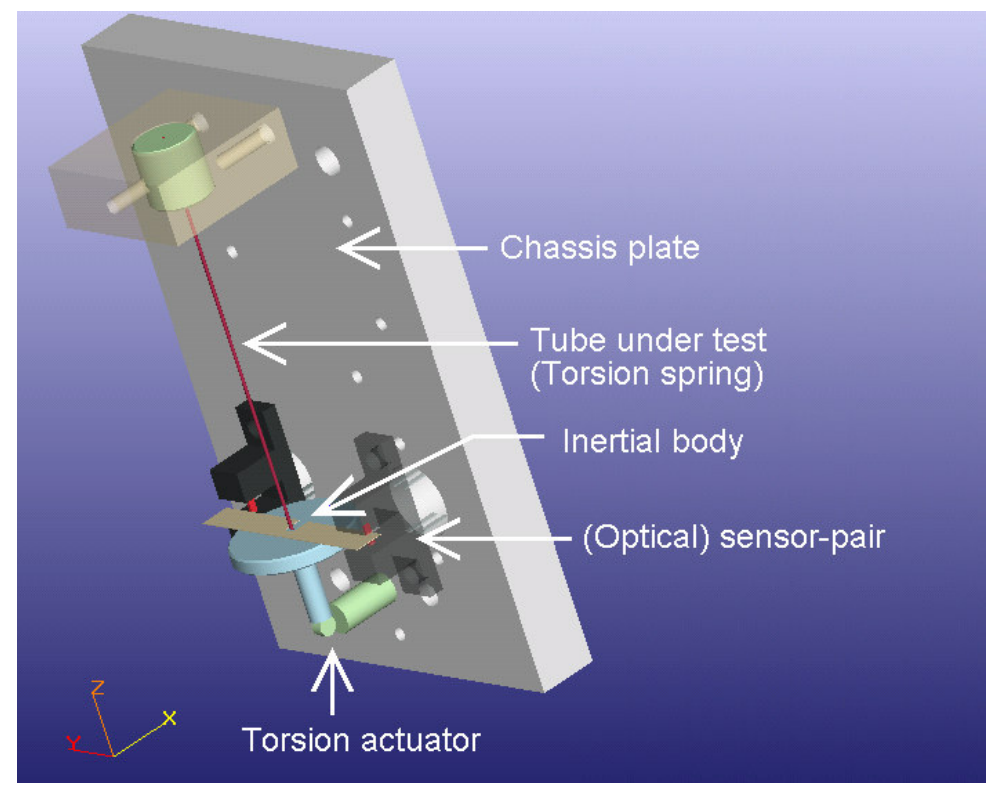

Figure 5.6 Experiment set up to determine temperature coefficient of stiffness

- The test setup consists of an SS tube-sample acting as a torsion-spring, rigidly connected to a chassis at one end.

- An inertial body (having a known moment of inertia) is attached to the other end of the tube. This body is actuated in a 'torsion' (Rz) direction, the actuator getting an excitation signal from an oscillator that observes the angular position of the body, and applies (effort) excitation at $90^{\circ}$ phase-shift, leading to excitation at eigenfrequency.

- As the shape of the inertial mass is well defined, the change in its moment of inertia due to temperature changes can be well predicted.

- The environment temperature can be regulated, and can be measured near the sample tube by means of a temperature sensor attached to the chassis.

- The (twist) eigenfrequency can be logged, measured against a crystal oscillator reference. Changes in the eigenfrequency can be related to the variation in torsionstiffness of the SS tube and the known change in the MI of the inertial mass.

Observations:

- The eigenfrequency is seen to change about $7.9 \mathrm{mHz} /{ }^{\circ} \mathrm{C}$ around $35^{\circ} \mathrm{C}$, for a nominal frequency of $29.3[\mathrm{~Hz}]$.

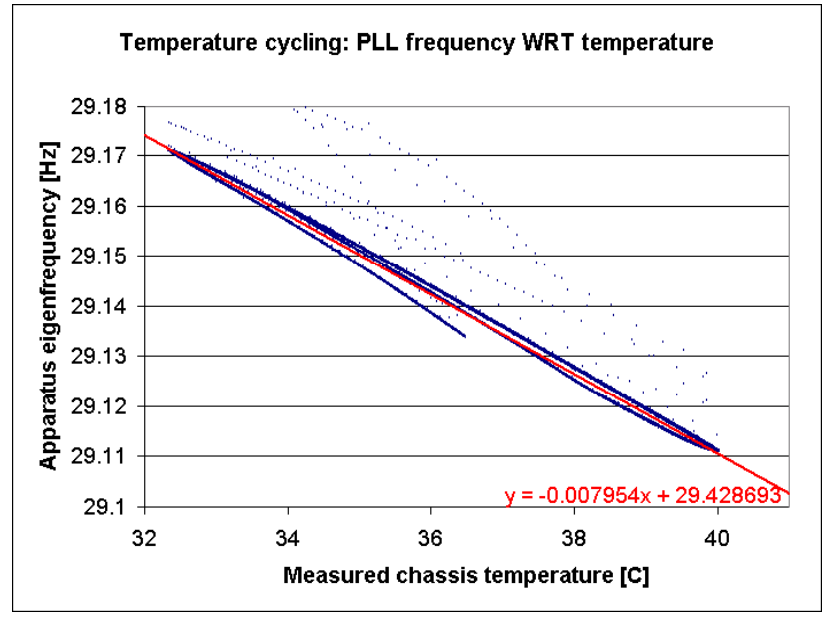

Figure 5.7 Observed change in oscillation eigenfrequency of the resonator from Figure 5.6 as a function of temperature 
This can be related to a change in stiffness as follows:

Table 5-1

\begin{tabular}{|c|l|c|c|l|}
\hline A & Inertial body & - & & Steel cylinder \\
\hline B & $\begin{array}{l}\text { Thermal expansion } \\
\text { coefficient of steel }\end{array}$ & $1.73 e-5$ & {$[\mathrm{~m} / \mathrm{m} \cdot \mathrm{K}]$} & At 20C \\
\hline C & $\begin{array}{l}\text { Thermal coefficient of } \\
\text { moment of inertia }\end{array}$ & $3.46 \mathrm{e}-5$ & {$\left[\left(\mathrm{~kg} \cdot \mathrm{m}^{2}\right) /\left(\mathrm{kg} \cdot \mathrm{m}^{2}\right) \cdot \mathrm{K}\right]$} & $\begin{array}{l}\text { For constant mass, varies as the } \\
\text { square of the diameter }\{\mathrm{C} \approx 2 \cdot \mathrm{B}\}\end{array}$ \\
\hline D & $\begin{array}{l}\text { Effect of thermal } \\
\text { coefficient of MI on } \\
\text { eigenfrequency }\end{array}$ & $-1.73 \mathrm{e}-5$ & {$[(\mathrm{~Hz}) /(\mathrm{Hz}) \cdot \mathrm{K}]$} & $\begin{array}{l}\text { Varies inversely as the square-root of } \\
\text { the moment of inertia } \\
\text { [D } \approx-\mathrm{C} / 2\}\end{array}$ \\
\hline E & $\begin{array}{l}\text { Measured deviation in } \\
\text { eigenfrequency }\end{array}$ & $-7.95 \mathrm{e}-3$ & {$[\mathrm{~Hz} / \mathrm{K}]$} & From experiments \\
\hline F & At nominal frequency & 29.3 & {$[\mathrm{~Hz}]$} & At 20C \\
\hline G & $\begin{array}{l}\text { Measured variation in } \\
\text { eigenfrequency }\end{array}$ & $-2.72 \mathrm{e}-4$ & {$[(\mathrm{~Hz}) /(\mathrm{Hz}) \cdot \mathrm{K}]$} & $\{\mathrm{E} / \mathrm{F}\}$ \\
\hline $\mathrm{H}$ & $\begin{array}{l}\text { Effect from other } \\
\text { sources (i.e. stiffness) }\end{array}$ & $-2.54 \mathrm{e}-4$ & {$[(\mathrm{~Hz}) /(\mathrm{Hz}) \cdot \mathrm{K}]$} & $\begin{array}{l}\text { Frequency varies as the square-root } \\
\text { of stiffness }\{\mathrm{G}-\mathrm{D}\}\end{array}$ \\
\hline I & $\begin{array}{l}\text { Thermal coefficient of } \\
\text { stiffness }\end{array}$ & $-5.08 \mathrm{e}-4$ & {$[(\mathrm{~N} \cdot \mathrm{m} / \mathrm{rad}) /(\mathrm{N} \cdot \mathrm{m} / \mathrm{rad}) \cdot \mathrm{K}]$} & $\begin{array}{l}\text { Stiffness, conversely, varies as the } \\
\text { square of eigenfrequency }\{\mathrm{I} \approx 2 \cdot \mathrm{H}\}\end{array}$ \\
\hline
\end{tabular}

The measured temperature dependence (coefficient) of stiffness, $-5.1 \mathrm{e}-4\left[\mathrm{~K}^{-1}\right]$, is a little more severe than that reported in literature for a different kind of steel $\left(-2.5 \mathrm{e}-4\left[\mathrm{~K}^{-1}\right]\right)$ (see [17]) or austenitic 316 steel: $-3.5 e-4\left[K^{-1}\right]\left([w w w]^{21}\right)$

or austenitic 316 steel: $-3.0 \mathrm{e}-4\left[\mathrm{~K}^{-1}\right]\left([\mathrm{www}]^{22}\right)$.

\subsubsection{Medium density and eigenfrequency}

The mass per unit length of tube comprises mostly the tube-wall material and thickness, and to some extent the tube-contents (i.e. the medium being measured). As the tube has a uniform cross-section throughout the construction, the inertia of the tube varies directly as the mass-per-unit-length of the tube.

Table 5-2

\begin{tabular}{|c|l|c|c|l|}
\hline A & Outer diameter of tube & $\boldsymbol{O D}$ & {$[\mathrm{m}]$} & \\
\hline B & Inner diameter of tube & $\boldsymbol{I D}$ & {$[\mathrm{m}]$} & \\
\hline C & Tube-wall cross section & $\boldsymbol{A}_{\boldsymbol{w}}$ & {$\left[\mathrm{m}^{2}\right]$} & $\left\{\pi / 4 \cdot\left(\mathrm{A}^{2}-\mathrm{B}^{2}\right)\right\}$ \\
\hline D & Density of tube material & 7960 & {$\left[\mathrm{~kg} / \mathrm{m}^{3}\right]$} & AISI 316 stainless steel \\
\hline E & Fluid channel cross-section & $\boldsymbol{A}_{\boldsymbol{c}}$ & {$\left[\mathrm{m}^{2}\right]$} & $\left\{\pi / 4 \cdot \mathrm{B}^{2}\right\}$ \\
\hline F & Density of fluid medium & $\rho_{\boldsymbol{m}}$ & {$\left[\mathrm{kg} / \mathrm{m}^{3}\right]$} & \\
\hline
\end{tabular}

The overall mass-per-unit-length of the tube is then $\{C \cdot D+E \cdot F\}[\mathrm{kg} / \mathrm{m}]$.

For a typical medium, air $\left(\rho_{\mathrm{m}}=1.3\left[\mathrm{~kg} / \mathrm{m}^{3}\right]\right)$, its value is $1.50 \mathrm{e}-3[\mathrm{~kg} / \mathrm{m}]$.

For another typical medium, water $\left(\rho_{\mathrm{m}}=1 \mathrm{e} 3\left[\mathrm{~kg} / \mathrm{m}^{3}\right]\right)$, it is $1.69 \mathrm{e}-3[\mathrm{~kg} / \mathrm{m}]$, i.e. a $13 \%$ increase. This is expected to lead to a decrease in eigenfrequency of about $6.5 \%$ due to the heavier medium. This prediction is seen to agree with experimental values for five fluids - air, methanol, ethanol, iso-propanol and water - to an extent that the density calculated from the measured eigenfrequency is within $+/-2\left[\mathrm{~kg} / \mathrm{m}^{3}\right]$ of the known value for gases and $+/-20$ $\left[\mathrm{kg} / \mathrm{m}^{3}\right]$ for liquids (see 6.5.4).

Knowing the effect of temperature on stiffness, the following procedure is proposed for estimating the medium-density in an actual instrument:

\footnotetext{
${ }^{21} \mathrm{http}: / /$ www.engineeringtoolbox.com/young-modulus-d_773.html

22 http://www-ferp.ucsd.edu/LIB/PROPS/PANOS/ss.html
} 


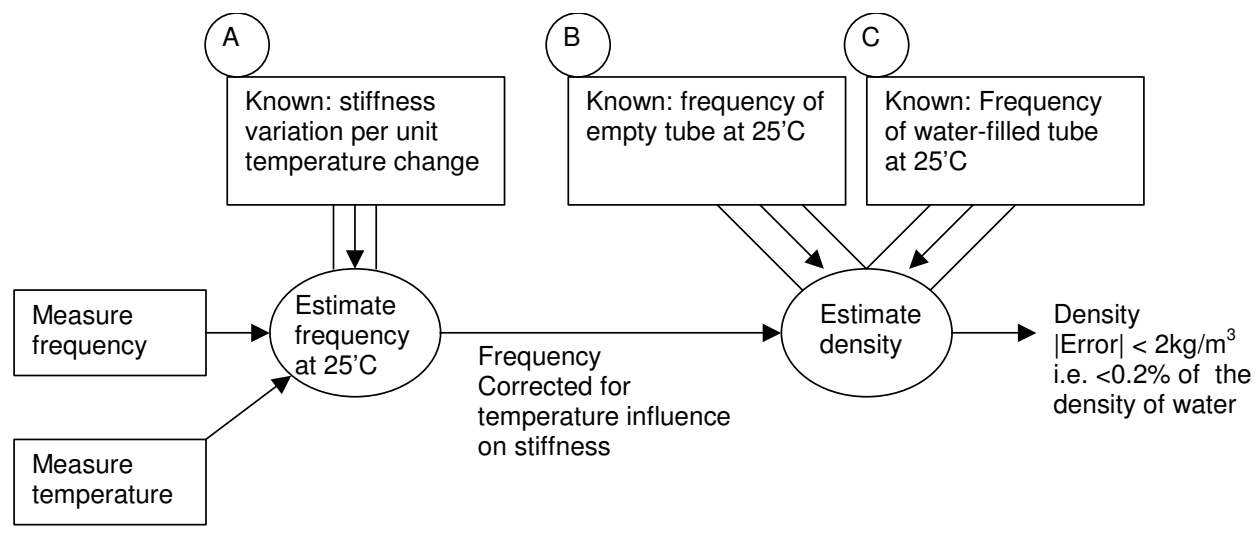

Figure 5.8 Procedure for estimating density

The three parameters $A B$ and $C$ need to be known to properly calculate the medium density. One way to know them is to measure each instrument at $25^{\circ} \mathrm{C}$ with air (B) and water (C) and then again at a higher temperature $\left(\right.$ say $65^{\circ} \mathrm{C}$ ) with air $(A)$. This procedure would be timeconsuming. It may be possible (depending on how identical individual instruments are) to use a fixed set of parameters (without individual calibration/setting). It is likely that the parameter A is identical for a set of instruments, if the tube-material is from the same batch. This means that the per-instrument high-temperature measurement may be omitted. The parameter $\mathrm{B}$ (eigenfrequency of empty tube at $25^{\circ} \mathrm{C}$ ) is probably not the same for various instruments due to constructional imperfections, and so has to be measured for each instrument. However, this parameter is simple to determine. The parameter $\mathrm{C}$ is also probably not constant; however the ratio $\mathrm{B} / \mathrm{C}$ probably is constant, as the exact inner diameter of tube from the same batch is likely to be identical. This can present a simple alternative to a second calibration run with water in the tube.

\subsubsection{Oscillator: Actuation at eigenfrequency}

As mentioned in 5.2.1, in order to get maximum tube excitation while applying a minimum effort, the tube must be excited at or very close to the eigenfrequency. The tube by itself is a passive mass-spring-damper system. As such, when given an initial energy (initial non-zero state), its motion is described by the damped harmonic oscillator (effort balance) equation:

$$
m \cdot \ddot{x}+R \cdot \dot{x}+k \cdot x=0
$$

The solution to such an equation is the well known "universal oscillator equation". Of the three 'efforts', i.e. inertial, resistive, and stiffness related, only the resistive contribution is dissipative, and leads to a decline in the energy stored in the mass-spring system. For systems with a high q-factor $(>10)$ the solution to this equation can be approximated by the product of an oscillatory part and an exponential-decay part, the overall motion looking like:

$$
x(t)=A_{0} e^{-R t / 2 m} \cos \left(\omega_{0} t+\phi\right)
$$

The term $2 m / R$ is the "decay time constant" mentioned in 5.2.1

Furthermore, the mean power dissipated by such a system -the average of the product of the instantaneous damping force and velocity- can be stated as:

$$
\langle P\rangle=\left\langle R \cdot\left(\frac{d x}{d t}\right) \cdot\left(\frac{d x}{d t}\right)\right\rangle
$$

At any given amplitude, the dissipation is proportional to the square of the amplitude. Left to its own means, the energy of this oscillating mass-spring-damper system would decay, like that of a pendulum or a tuning fork after a single hit. However, if by means of an actuator, the same power (as that being dissipated at a particular amplitude) is fed into the oscillating 
system, the actuation will compensate for the dissipation, and stable (constant "dx/dt") oscillations will result.

\section{Actuation strategy - Part 1 of 2: the nature of excitation}

Let us first consider the motion of the oscillating tube on a short time-scale $T$, such that for the tube eigenfrequency $f_{\text {eig }}=\frac{1}{2 \pi} \sqrt{\frac{k}{m}}$ and tube oscillation decay time-constant $\tau=\frac{R}{2 \cdot m}$, the relation $\frac{1}{f_{\text {eig }}}<T<\tau$ holds.

The instantaneous power can be described as the dot product of the instantaneous velocity and the instantaneous force (angular velocity and torque, in our case). In order to feed power into the oscillating system, the average applied power (average of instantaneous power over one or more cycles) should be positive. In other words, the applied torque should be in-phase with the actual angular velocity of the twist-oscillating tube.

We have chosen for optical occlusion type position sensors in [4.3.2]. These give a representation of the instantaneous (angular) position of the oscillating tube, not the velocity. If the angular position is represented as $\theta(t)$, then:

$$
\theta(t)=A \cos \left(\omega_{0} t+\phi\right)
$$

$A$ being the (slowly varying) oscillation amplitude.

The angular velocity of the tube has a definite phase-relation to the angular position - it leads the position by phase $\pi / 2$. Additionally, we know the approximate eigenfrequency of oscillation, using which the $\pi / 2$ (angle) may be translated to a time-delay (seconds). We can thus use the measured position signal, with a phase-shift $(-3 \pi / 2$ instead $+\pi / 2$ for causality), to guess the phase of the angular velocity:

$$
\dot{\theta}(t)=-A \omega_{0} \sin \left(\omega_{0} t+\phi\right)=A \omega_{0} \cos \left(\omega_{0} t+\phi-3 \pi / 2\right)=\omega_{0} \cdot(\theta(t) \angle-3 \pi / 2)
$$

Given that the Q-factor of the tube is quite high, this guess is used to generate an effort (torque) at an appropriate phase so as to feed power into the oscillatory system. The $-3 \pi / 2$ radian delay can be conveniently generated by means of a (digital) delay line, implemented as a ring-buffer with a variable pick-off-point.

It is not essential for the phase of this guess to correspond precisely to the phase of the actual velocity; an error in the phase will lead to an effort that is off by a phase, say $\Delta$. This error will be resolved into sine and cosine components, the cosine component ( $\cos \Delta \cong 1$ ) will feed power, while the sine component ( $\sin \Delta \cong 0$ ) will have no long-term effect on the energy of the system. Note here that an error in guessing the initial frequency leads to a temporary phase error in the actuation, not a frequency error. This phase error is progressively removed, as the exact eigenfrequency of the oscillator becomes known, once it starts to oscillate.

\section{Actuation strategy - Part 2 of 2: the magnitude of excitation}

Next, consider a long time-scale $T *$ such that given the decay time-constant $\tau=\frac{R}{2 \cdot m}$,

the relation $T^{*}>\tau$ holds.

On this time-scale, the amplitude $A$ (of angular displacement of the system) can be stated as

$$
A(t)=A_{0} e^{-R t / 2 m}=A_{0} e^{-t / \tau}
$$


$A(t)$ being the amplitude of $x(t)$ in equation 5.16 , with the oscillatory portion ignored. This is a simple $1^{\text {st }}$ order "exponential decay" function. Similarly, the energy (which is proportional to the square of the magnitude) can also be thought of as an exponential decay, only with a time-constant that is half as much. The amplitude of the oscillator can be made to track a set value by means of a simple I or P+I type controller with the following caveat:

The "amplitude" that is being set can be easily measured only at peak-values, thus this quantity is not continuous, but some form of "peak detection" scheme is required. This involves a state that "remembers" the maximum attained excursion in the recent past. Such a peak repeats only $2 \cdot f_{\text {eig }}$ times per second, and hence the "remember" function (some form of low-pass filter) must have a time constant that is slower than the repetition rate of the peak. Consequently, the P+I controller settings should be slower still than the low-pass filter.

The output of the controller is a value representing the required effort. However, the oscillation is a cyclic phenomenon, and the effort to be applied too must be cyclic, at the appropriate phase. The $-3 \pi / 2$ [rad] phase-shifted position signal can be used to this end. A sinusoidal reference signal at the appropriate phase can be derived from this signal by dividing the waveform by the (measured) amplitude. This unit-waveform is scaled by the output of the controller, and fed to the actuator.

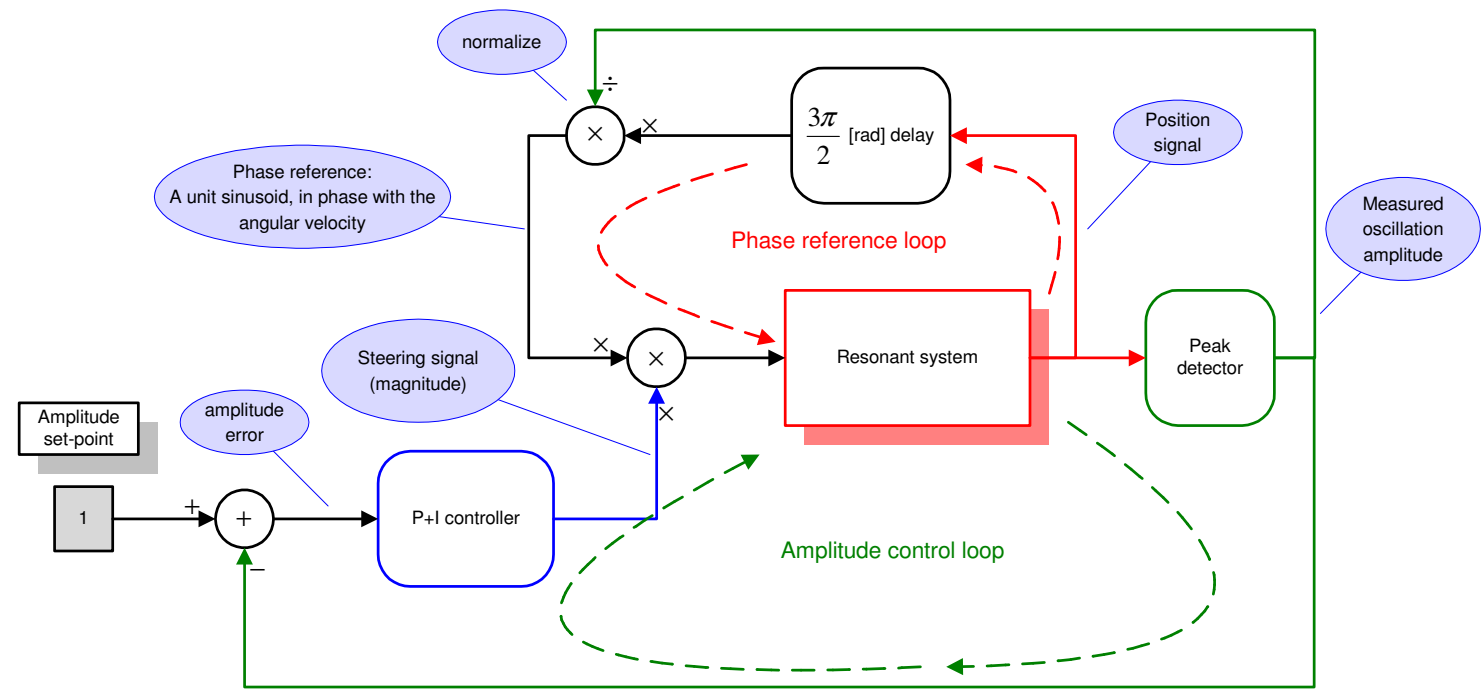

Figure 5.9 Oscillator for a resonant system

\subsection{Sensing the Coriolis effect}

Mass-flow through the tube (developed in 5.1) that oscillates (as per 5.2) leads to the generation of Coriolis force in its rotating parts. As outlined in Ch. 2.1, the Coriolis force can be expressed as

$$
\widetilde{\bar{F}}_{c}=2 l \cdot \bar{\Phi}_{m} \times \widetilde{\widetilde{\theta}}
$$

The symbols have their usual meanings. The 'tilde' symbols on the Coriolis force $F_{c}$ and the angular velocity $\dot{\theta}$ are a reminder that due to the oscillatory nature of the instrument, these quantities too are oscillatory. Note that the Coriolis force is in-phase with the instantaneous excitation angular velocity and hence exactly $\pi / 2$ [rad] out-of phase with the excitationdeflection. 
Depending upon the geometry of the sensor tube, the Coriolis force will cause a 'response' motion, whose magnitude will be proportional, among others, to the mass flow rate through the tube and the angular velocity of the (rotating) tube. The interaction of the various tube parameters and quantities is depicted in the following model:

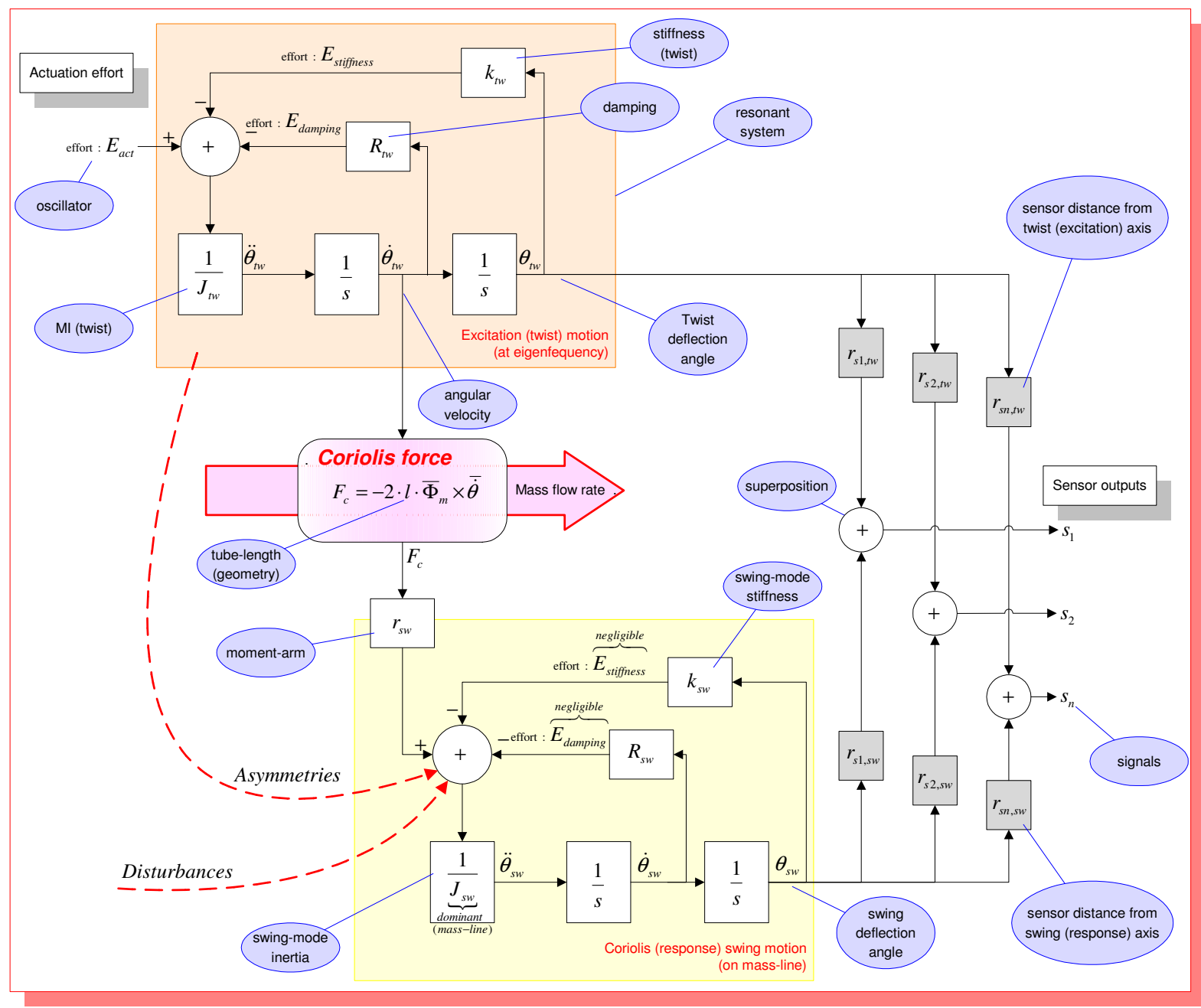

Figure 5.10 Parameters' \& quantities' interaction model of a Coriolis tube having the response mode on the mass-line

A parametric calculation-sheet (with heights, widths, E-moduli etc. as the inputs) is used for the purpose of estimating the various model parameters (torsion stiffness, moment arm etc). Using the two together, the various system goals (e.g. a large amplitudes' ratio on the sensor outputs) are sought.

As an illustration, the operational orders-of-magnitude of a type-II instrument are presented:

\subsubsection{Magnitudes}

Table 5-3 Typical magnitudes in a type-II instrument

\begin{tabular}{|l|l|l|l|l|}
\hline A & $\begin{array}{l}\text { Typical FS mass-flow rate } \\
\text { (type II instrument) }\end{array}$ & $2.78 \mathrm{e}-5$ & {$[\mathrm{~kg} / \mathrm{s}]$} & i.e. $100 \mathrm{~g} / \mathrm{h}$ \\
\hline B & Active rotating length & $4 \mathrm{e}-2$ & {$[\mathrm{~m}]$} & i.e. $40 \mathrm{~mm}$ \\
\hline C & Oscillation frequency & $f_{\text {eig }}$ & {$[\mathrm{Hz}]$} & Calculated by FEM; agrees with proto-measurements \\
\hline D & Angular excursion & $2.8 \mathrm{e}-2$ & {$[\mathrm{rad}]$} & Amplitude of excitation $\theta_{\text {max }}$ \\
\hline
\end{tabular}




\begin{tabular}{|l|l|l|l|l|}
\hline $\mathrm{E}$ & Angular velocity (amplitude) & 28 & {$[\mathrm{rad} / \mathrm{s}]$} & $\begin{array}{l}\dot{\theta}_{\max }, \text { given that } \theta(t)=\theta_{\max } \sin \left(2 \pi \cdot f_{\text {eig }} \cdot t\right) \\
\{2 \pi \cdot \mathrm{C} \cdot \mathrm{D}\}\end{array}$ \\
\hline $\mathrm{F}$ & Typical Coriolis force & $6.2 \mathrm{e}-5$ & {$[\mathrm{~N}]$} & $\begin{array}{l}\text { See fig. 5.9 - An antiparallel pair originates in the top } \\
\text { and bottom sections of the tube, causing the tube to } \\
\text { 'swing' }\end{array}$ \\
\hline
\end{tabular}

Innovation: Placement of sensors: In 4.4, we have chosen for measuring the Coriolis force in terms of phasors, by observing the superimposed motion of the excitation and that due to Coriolis force. In order not to get too large a displacement due to the excitation, we should measure close to the excitation (vertical) axis. As the rotation axis of the swing motion lies close to the upper horizontal tube section, there will be little "Coriolis displacement" here. By elimination, the place to measure (the displacement of) the tube is on the bottom horizontal section, close to the excitation axis, as shown.

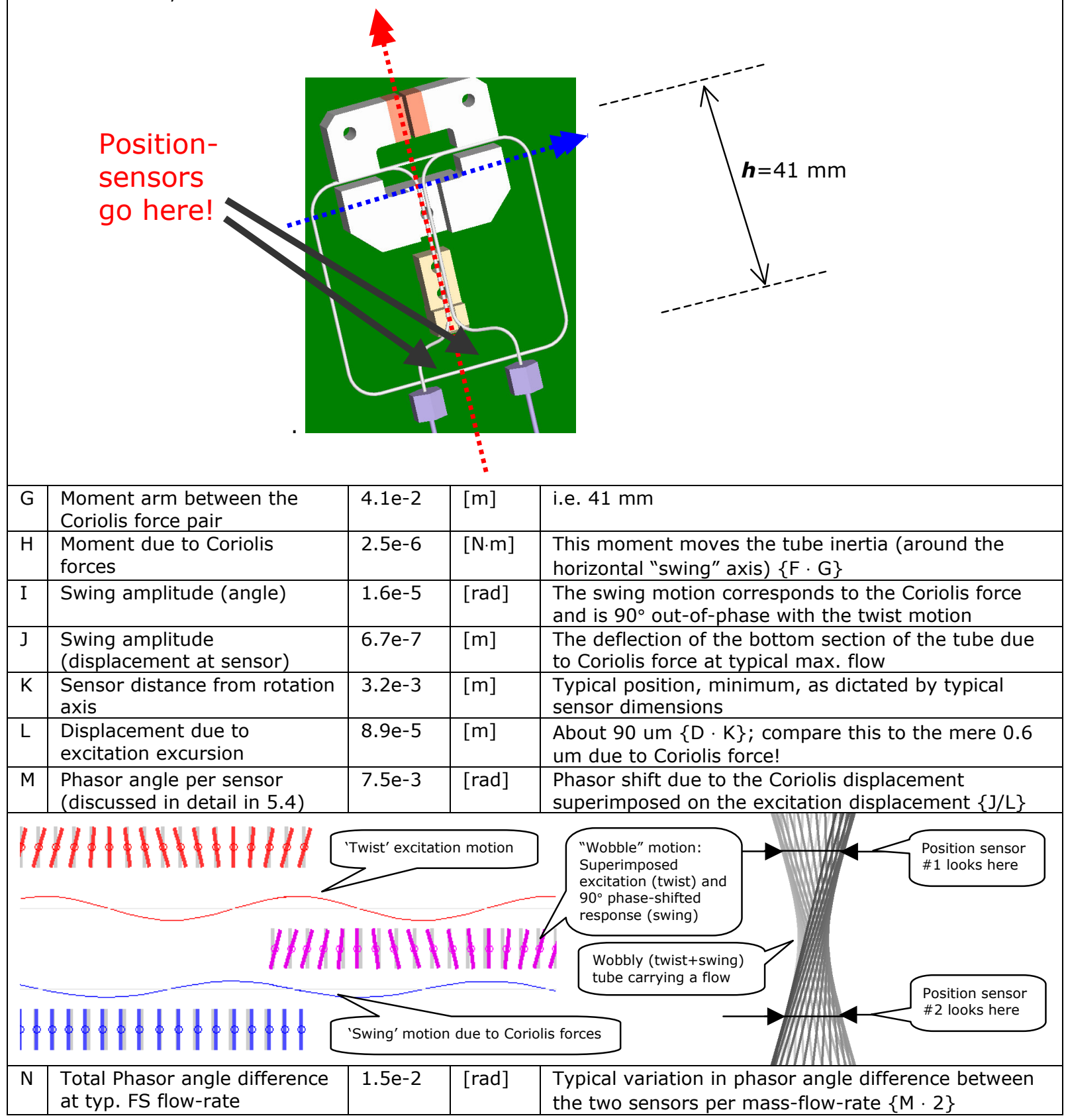




\subsubsection{Variation in sensitivity}

5.3.1 gives an idea about the sensitivity expected from a typical type-II instrument. The exact sensitivity can, if needed, be measured per instrument on a calibration tool, and accordingly adjusted. However, as discussed earlier in the 5.2.4, the tube eigenfrequency varies with temperature (stiffness of the tube) and with the medium density (inertia). We attempt here to develop an analytical expression for the change in sensitivity as a function of these two parameters (adapted from [12]).

\section{A quick summary (reiteration) of the critical points:}

The Coriolis meter under discussion in the following subsections is assumed to have the eigenfrequency of excitation (periodic rotation motion) higher than the eigenfrequency of the native mode of the resultant ('Coriolis') motion. This means that the 'Coriolis motion' is driven on its mass-line, with a phase-shift of $180^{\circ}$ between the excitation (periodic Coriolis force) and the position.

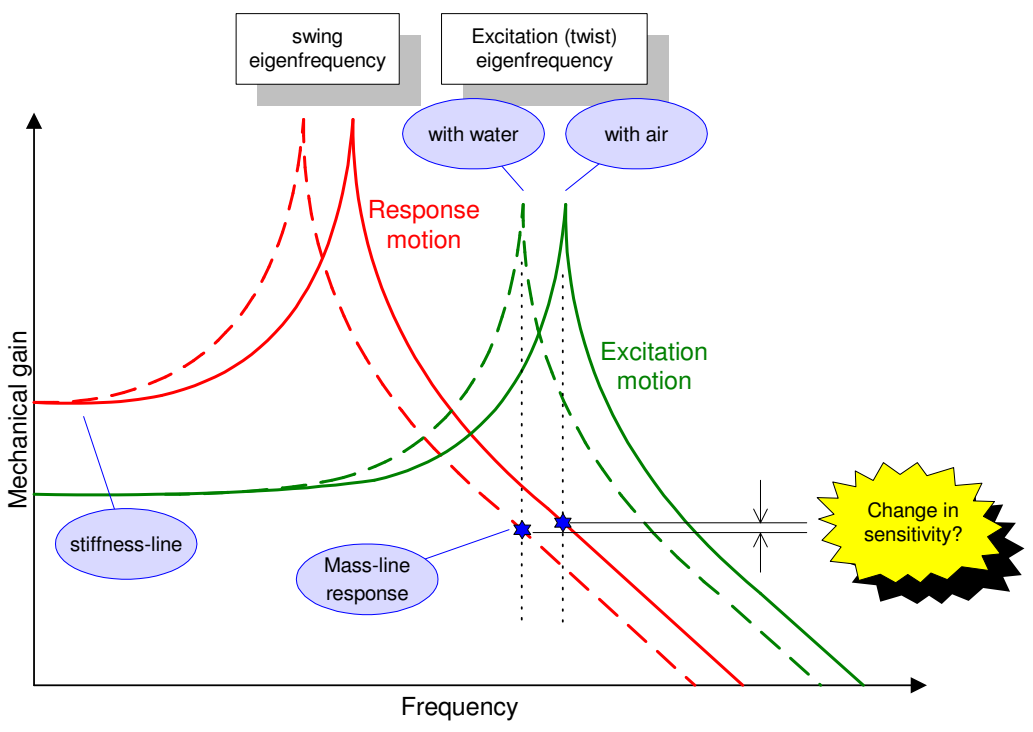

Figure 5.11 Change in sensitivity due to medium - an illustration

A change in either of the response curves (swing mode or excitation twist mode) can possibly lead to a change in the sensitivity of the instrument to the generated Coriolis force.

\section{Correction for a change in medium (fluid) density:}

When the density of the medium inside the Coriolis tube changes, two (relevant) things happen - the equivalent swing inertia $J_{s w}$ of the tube changes (affects mass line of response mode) and the equivalent twist inertia $J_{t w}$ also changes (affects eigenfrequency of excitation mode). The Coriolis tube is essentially a steel tube with masses (vanes, solder joints etc.) attached to it at various points. It has a particular shape. The fluid medium inside contributes a little to the final values of $J_{s w}$ and $J_{t w}$, and not identically. The effects of such a change in medium are illustrated below (using example media \& an exaggerated effect): 
Table 5-4 Effect of medium-density change

Excitation amplitude

$\theta_{t w}$

Angular velocity and Coriolis force

$\dot{\theta}_{t w}, F_{c}$

Swing-mode acceleration due to Coriolis force
The excitation (twist) amplitude $\theta_{t w}$ is held constant by the amplitude control loop. The excitation frequency (i.e. twist mode eigenfrequency) however, changes inversely as the root of the moment of inertia $J_{t w}$

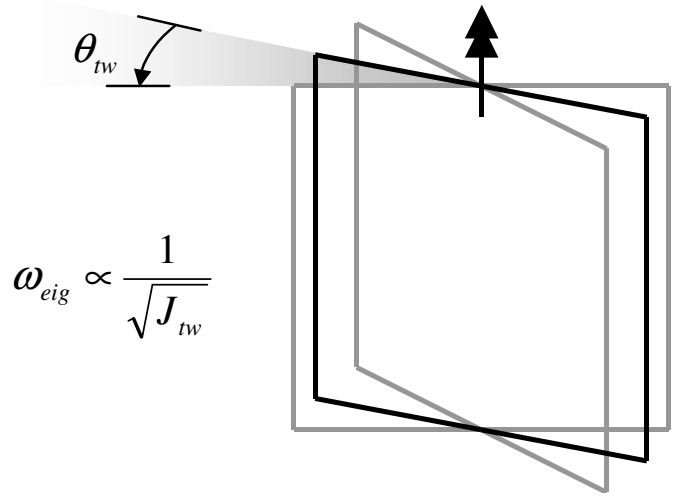

Lighter medium; higher eigen-

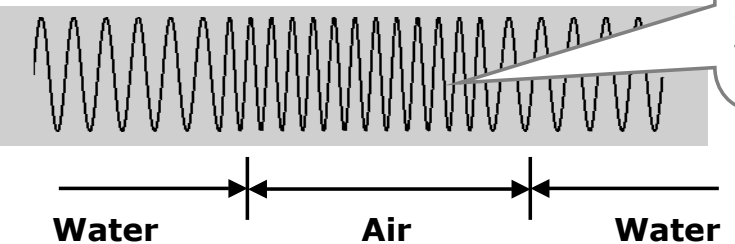

$$
J_{s w(\text { water })}, J_{t w \text { (water })} J_{s w(\text { air })}, J_{t w(\text { air })} \quad J_{s w(\text { water })}, J_{t w \text { (water })}
$$

The instantaneous angular velocity $\dot{\theta}$ of the tube changes as the oscillation eigenfrequency $\omega_{\text {eig }}$; so does the Coriolis force.

$$
\begin{aligned}
& \text { WNMNMMAMMMMMNMN } \\
& \dot{\theta}_{t w(\text { peak })}=\left(\frac{d \theta_{t w}}{d t}\right)_{\text {peak }} \propto \frac{1}{\sqrt{J_{t w}}} \\
& F_{c(\text { peak })}=\left(2 \cdot l \cdot \Phi_{m} \times \dot{\theta}_{t w}\right)_{p e a k} \propto \frac{1}{\sqrt{J_{t w}}}
\end{aligned}
$$

The 'Coriolis acceleration' varies as the Coriolis force $F_{c}$, and inversely as the swing inertia $J_{s w}$, which also changes with medium-density

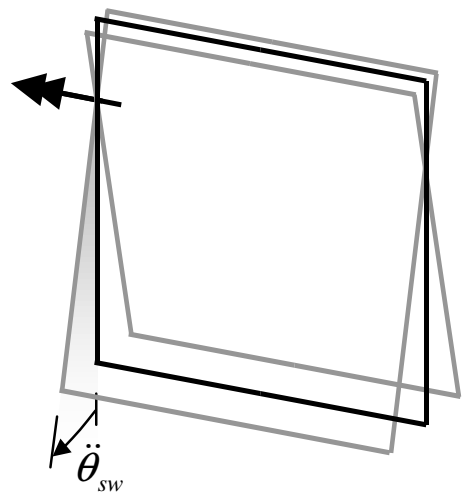




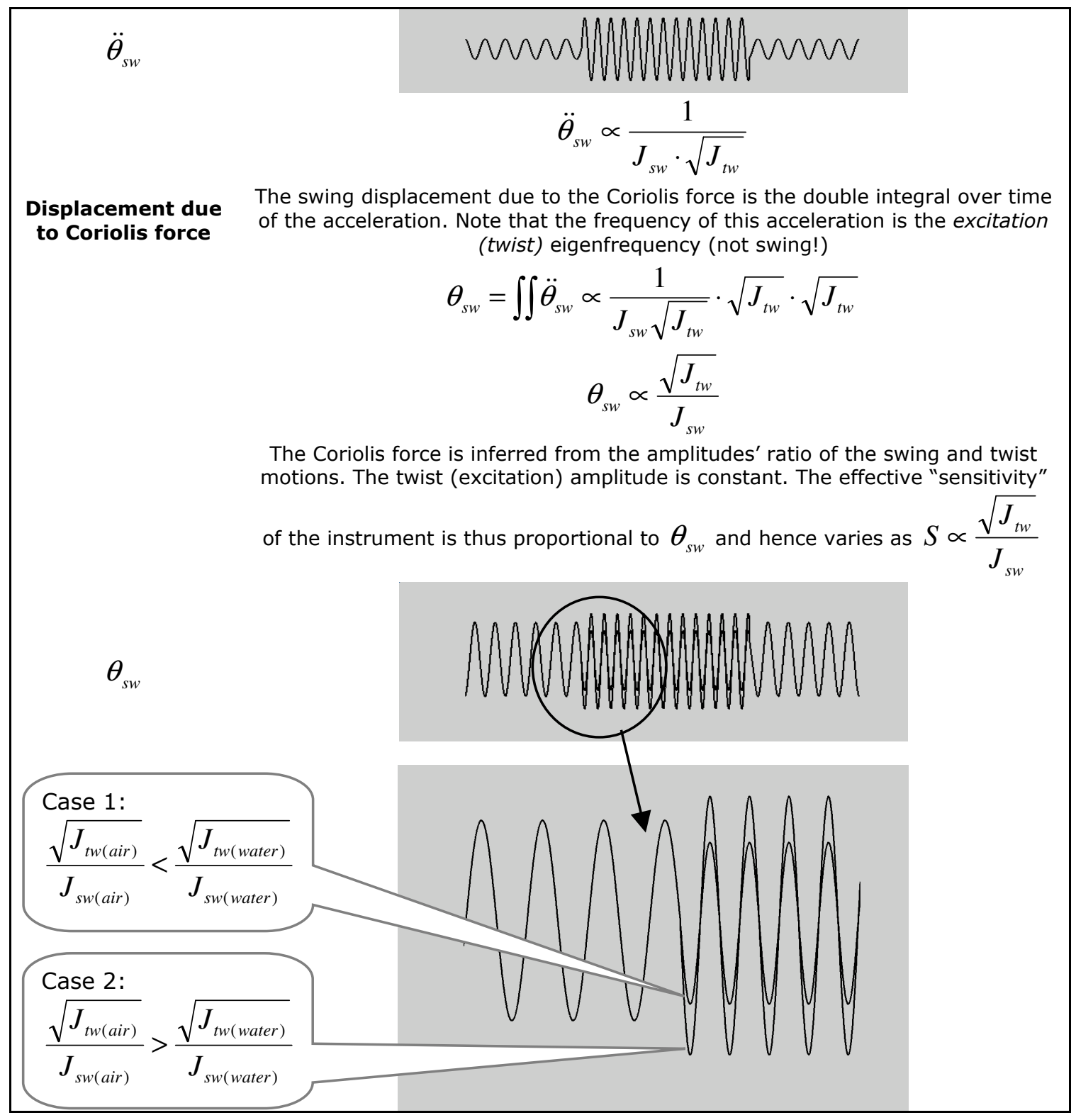

It is thus seen that the sensitivity, for a given medium $x$ varies as:

$$
S_{x} \propto \frac{\sqrt{J_{t w(x)}}}{J_{s w(x)}}
$$

The ratios of measured eigenfrequencies can be used to determine whether $S_{\text {air }}>S_{\text {water }}$.

The twist mode (excitation) eigenfrequency is given by:

$$
\omega_{\text {eig(air)tw }}=\sqrt{\frac{k_{t w}}{J_{t w(\text { air })}}} \text { and } \omega_{\text {eig(water)tw }}=\sqrt{\frac{k_{t w}}{J_{t w(\text { water })}}}
$$


Of these, if we assume that the torsion stiffness $k_{t w}$ remains constant (though unknown) for a given temperature, and if we accurately measure the twist eigenfrequencies of various modes, we have:

$$
\sqrt{\frac{J_{t w(\text { water })}}{J_{t w(\text { air })}}}=\frac{\omega_{\text {eig (air }) t w}}{\omega_{\text {eig(water }) t w}}
$$

Similarly, it may be shown that the eigenfrequency of the response 'swing' mode, for a given temperature (i.e. constant stiffness) has the relation:

$$
\frac{\omega_{\text {eig (air)sw }}}{\omega_{\text {eig (water }) s w}}=\sqrt{\frac{J_{\text {sw(water })}}{J_{s w(\text { air })}}}
$$

in other words,

$$
\frac{J_{\text {sw(water })}}{J_{\text {sw(air })}}=\left(\frac{\omega_{\text {eig }(\text { air }) s w}}{\omega_{\text {eig }(\text { water }) s w}}\right)^{2}
$$

From equations 5.22, 5.24 and 5.26, we may determine the ratio of the instrument sensitivities for air and water:

$$
\frac{S_{\text {air }}}{S_{\text {water }}}=\frac{\sqrt{J_{t w(\text { air })}}}{\sqrt{J_{t w(\text { water })}}}: \frac{J_{s w(\text { air })}}{J_{s w(\text { water })}}=\frac{\omega_{\text {eig(water }) t w}}{\omega_{\text {eig(air)tw }}}:\left(\frac{\omega_{\text {eig (water }) s w}}{\omega_{\text {eig(air)sw }}}\right)^{2}
$$

For any given medium, the swing and twist eigenfrequencies vary as a function of the density. This function can be determined from two measurements with reference media (say air and water) at the time of calibration. During operation, the medium density can be calculated based upon the twist (excitation) eigenfrequency and the tube temperature (both of which can be directly measured), as discussed in 5.2.4 and 5.2.5. Now, using the measured relation, the swing eigenfrequency can be determined from the calculated density. The two eigenfrequencies (twist and swing) can be used to predict the change in sensitivity of the instrument as a consequence of a change in medium density as per Eq. 5.27.

In Ch. 6.5, we show that data measured from a prototype setup agree with the expected sensitivity variations.

\section{Correction for change in sensitivity due to temperature variations:}

By the similar arguments as in Table 5-4, the expected change in sensitivity due to the temperature variation (change in stiffness) may be calculated. However, there is only one variable (torsion stiffness) that affects the sensitivity, as the Coriolis response lies on the mass line of the response (swing) mode (and thus a change in swing-stiffness has no effect). Let us consider an example where:

1. The temperature increases, thus the excitation mode (torsion) stiffness decreases.

2. The eigenfrequency decreases as the inverse square root of stiffness, as does the Coriolis force, for the same actual mass flow (and medium).

3. Although the Coriolis force decreases, the period for each cycle $\left(1 / 2 \pi \omega_{\text {eig }}\right)$ increases. As a combined effect, the displacement (which is the double integral of acceleration caused by the Coriolis force) increases as the period.

$$
S_{(T)} \propto \frac{1}{\omega_{e i g(T)}}
$$


4. In other words, for a change in the temperature $T$ causing a variation in sensitivity $S$, we have:

$$
\frac{S_{\text {hot }}}{S_{\text {cold }}} \propto \frac{\omega_{\text {eig (cold })}}{\omega_{\text {eig (hot })}}
$$

This rule too agrees well with measurements in Ch. 6.5. The dimensional expansion due to temperature, which would also affect the eigenfrequencies, is also included here. However, as seen in Table 5-1, it has a significantly smaller contribution than the change in materialstiffness.

\subsection{Processing - Ratiometric measurement from phasor angles}

In 5.3.1 and Appendix $A$, a manner of identifying an out-of-phase swing motion superimposed on a twist (excitation) motion has been briefly discussed. Basically, we have a meter tube with an oscillatory angular-deflection excitation of $\theta_{\max }$, at the twist-eigenfrequency of the tube.

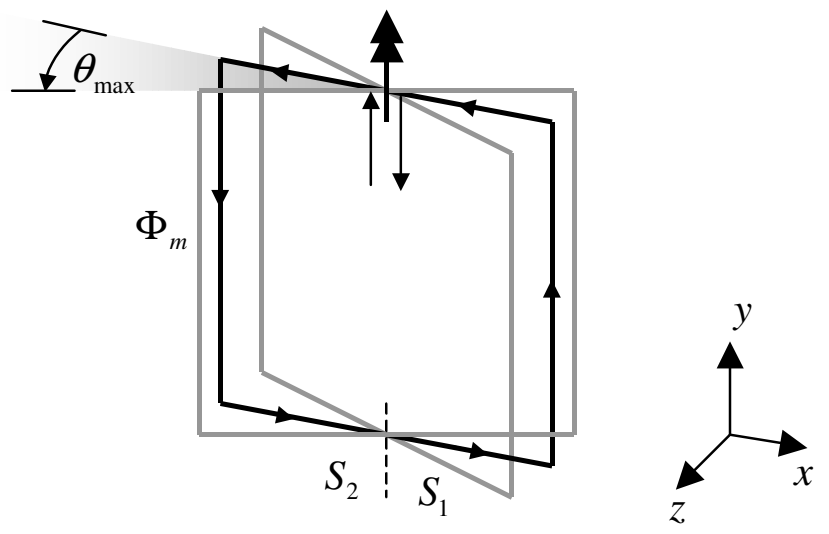

Figure 5.12 Axes convention

Two position sensors placed at distances $\left|x_{s 1}\right|$ and $\left|x_{s 2}\right|$ from the rotation pole observe the meter tube. Typically $x_{s 2}=-x_{s 1}$. The sensors monitor tube-position in the z-direction. The tube has an angular excursion:

$$
\theta(t)=\theta_{\max } \cos \left(\omega_{t w} \cdot t\right)
$$

with some arbitrary phase, which we ignore (treat as zero). Let us treat this signal as the reference.

Due to the angular excursion of the meter tube, the sensors observe:

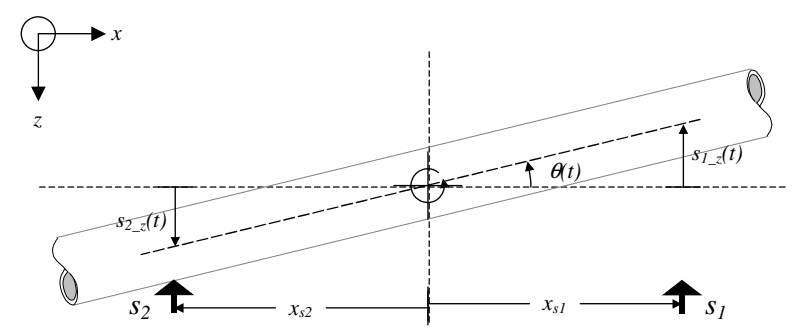

Figure 5.13 Excitation motion as seen by the position-sensors 


$$
s_{1_{-} z}(t)=x_{s 1} \cdot \tan (\theta(t)) \cong x_{s 1} \cdot \underbrace{\theta(t)}_{\text {for small } \theta}
$$

and

$$
s_{2_{-} z}(t)=x_{s 2} \cdot \tan (\theta(t)) \cong x_{s 2} \cdot \theta(t)
$$

These signals can be represented as the phasors $s_{1 z}$ and $s_{2 z}$ in Figure 5.14

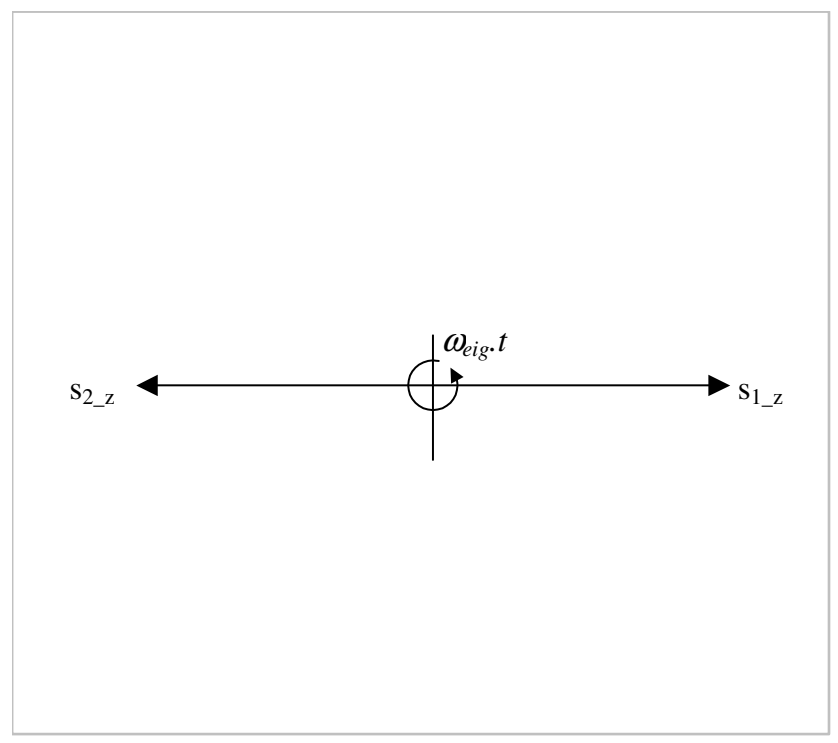

Figure 5.14 Phasors: two ideal sensors observe pure excitation motion

Here,

- $\quad$ The s1 and s2 signals are exactly anti-phase (as the sensors $S_{1}$ and $S_{2}$ are on either side of the rotation axis)

- $\quad$ They are equal, as the sensors are identical and equidistant from the rotation pole.

- The phasors are cyclic at the twist (excitation) eigenfrequency of the tube; the phase $\psi$ of the phasor [rad] is not to be confused with the angular deflection $\theta$ of the tube [rad].

- The angular velocity of rotation of the tube ( $d / d t$ of the angular position) has a phasor that leads the position-phasor by $90^{\circ}$ (i.e. $\pi / 2$ rad).

A mass flow through the tube leads to generation of Coriolis force in phase with the angular velocity. The Coriolis force too then has a phasor that leads the position-phasor by $90^{\circ}$. This force acts along the entire length of the rotating tube. In the example construction in Figure 5.12 , there are two rotating tube sections, a top and a bottom section, each with a flow in opposing directions. Each thus has Coriolis force (anti-parallel pair) leading to a couple. This couple tries to move the tube in a "swing" motion. As outlined in 4.1 and 5.1.2, the swing mode eigenfrequency is intentionally chosen to lie away from the twist excitation eigenfrequency. In our example, the couple caused by the Coriolis force causes a response on the mass line as $f_{\text {eig(response) }}<f_{\text {eig(excitation) }}$. The response (swing) motion can thus be stated as $\ddot{\theta}_{s w}=\frac{\mathrm{T}_{c}}{J_{s w}}$. The swing motion lags the Coriolis-force-phasor by $180^{\circ}$ (i.e. $\pi$ [rad]). In the ideal situation, the swing motion causes an equal displacement on each sensor, giving phasors as per Figure 5.15. 


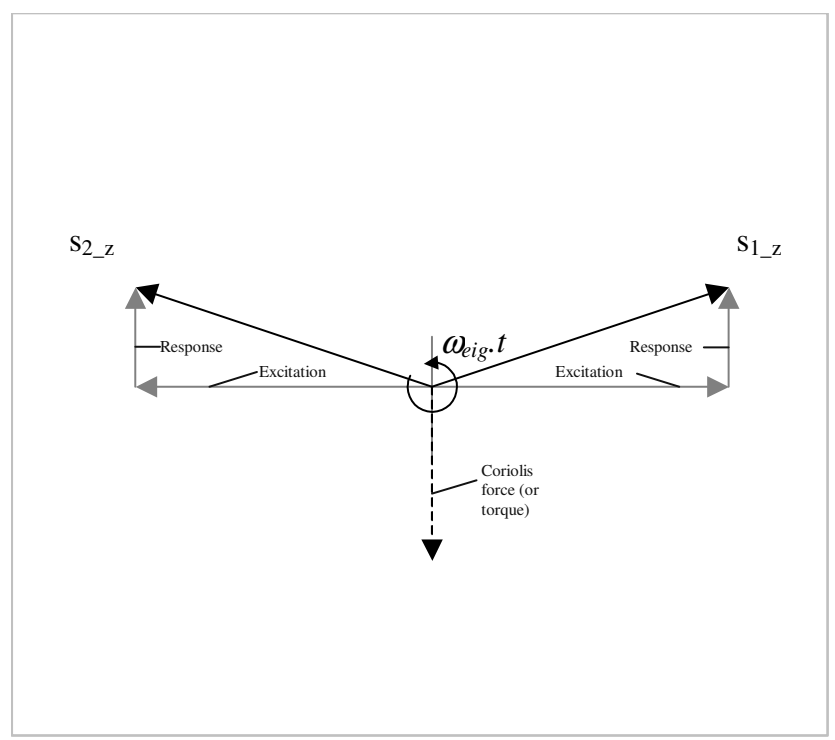

Figure 5.15 Phasors: two ideal sensors observe excitation + Coriolis (swing) response

By means of phase analysis of the sensor signals, it is possible to determine the angle (deviation from $180^{\circ}$ ) between the $s_{1 \_z}$ and $s_{2 \_z}$ signals. The quantity that we are ultimately interested in is the mass flow rate. Having chosen for a concept that uses only time domain measurements for the determination of mass-flow-rate, let us consider how this may be implemented using the phase information from the signals:

The swing motion, superimposed upon the excitation motion, leads to a "wobbly" motion as depicted in Table 5-3, represented in the phasor in Figure 5.15. The sensor S1 reads a position $\mathrm{s}_{1 \_z}$ that is phase-shifted from the case where purely an excitation was present; however, this information cannot be gathered from looking at S1 alone. Similarly, the swing also affects the motion observed by S2; only this time, the phase-shift is in the opposite sense.

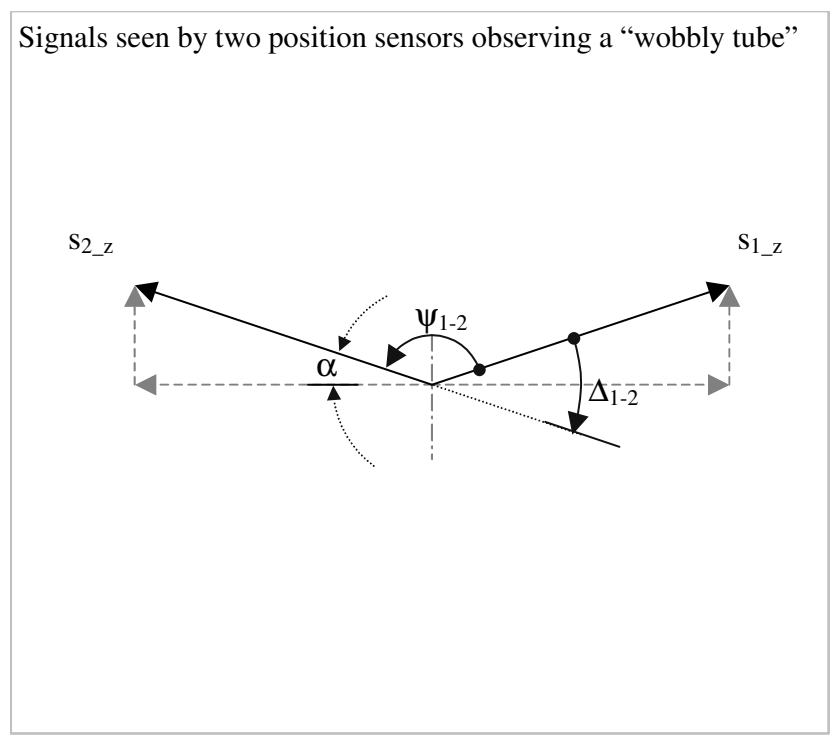

Figure 5.16 Phasor: signals seen by two position sensors observing the wobbly motion

The result is that the (measurable) phasor-angle $\psi_{1-2}$ is no longer $180^{\circ}$ ( $\pi$ rad) but has changed by an angle $\Delta_{1-2}$. When the two sensors are ideal, this angle is twice the angle $\alpha$, the shift in each phasor (see Figure 5.16). The extent-of-wobble i.e. the ratio of swing and twist motion (as observed by the position sensors) is then the tangent of $\alpha$. For small angles, 
as $\tan \alpha \approx \alpha$, we can draw an approximate relation between the measured phase-shift ( $\left.\Delta_{1-2}\right)$

and the flow.

In 5.3, we have discussed, in effect, the various corrections to deduce Coriolis force from a wobble and then mass-flow-rate from the Coriolis force. Here, we will restrict the discussion to the determination of wobble itself.

\subsubsection{Sources of errors}

We have made some simplifying assumptions to arrive at the phasor in Figure 5.16. Lessthan-ideal, circumstances (where these assumptions do not hold) can cause an error in the estimation of the wobble. These are:

Unequal phase delays in sensors S1 and S2:

Say that the tube moves ideally; still, the readout electronics or the sensors introduce unequal (or -even worse- time varying) phase delays in the position measurement. The phase difference adds to $\psi_{1-2}$ and is incorrectly interpreted as wobble. (Figure 5.17)

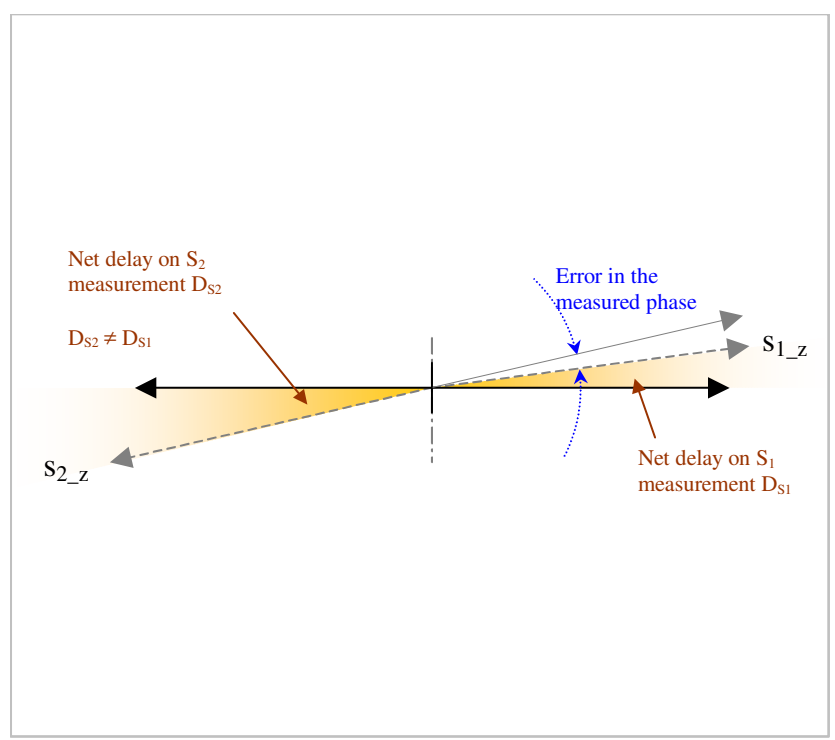

Figure 5.17 Phasor: Difference in measurement-delay leads to error in measured phase (exaggerated)

This source of error has been mitigated by the concept choice of using optical pickups with transimpedance amplifiers ${ }^{23}$. Depending upon the choice of components, such a combination leads to a delay (between the instant that light is incident upon a sensor and the instant that the incident light appears as an electrical signal). Typically, this delay is a few tens of nanoseconds, the inter-sensor mismatch being a fraction ( $20 \%)$ of this delay. The leastcount of a typical instrument corresponds to a phasor angle (see Table 5-3) of about $15 \mu$ rad. This means that the few nanosecond delay-difference between two sensors causes a phasorangle-difference that is smaller than the least-count, and hence negligible.

\section{Unequal gains of sensors $\mathbf{S 1}$ and S2:}

In the concept (Ch. 4.4) of estimating Coriolis force solely based upon time (or phase) measurements, we have chosen to neglect all amplitude information. Unequal or varying gains of S1 and S2 should, then, not cause an error in measurement. Consider Figure 5.18.

${ }^{23}$ See Appendix C 


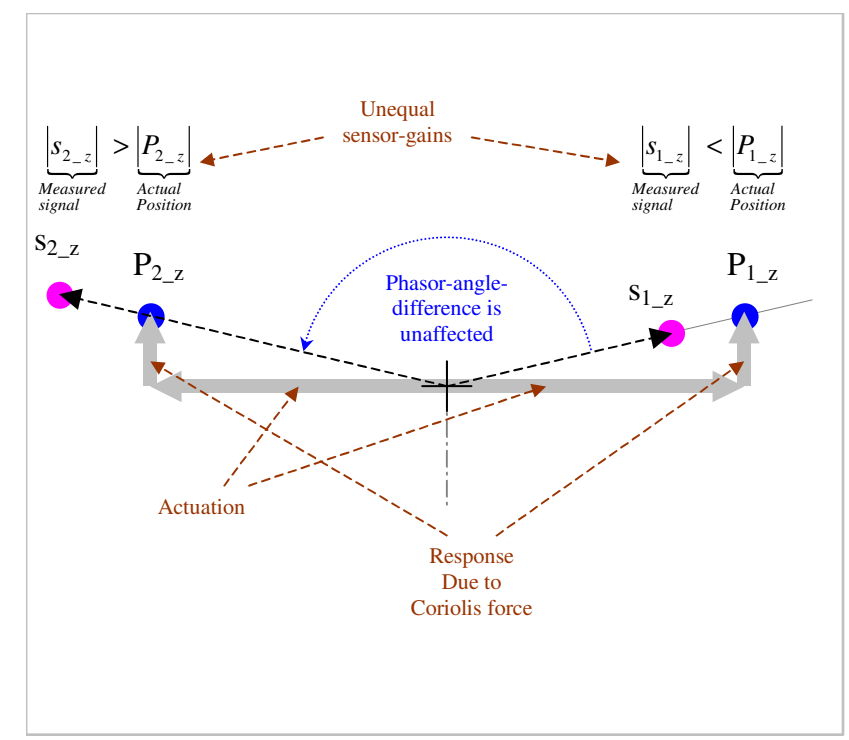

Figure 5.18 Phasor: Dissimilar sensor gains have no effect on phase-difference

The z-position of the tube varies due to wobble as before. The sensors, however, have unequal gains. They give out unequal (measured) amplitudes. However, the phase relation between the two signals $s_{1 \_z}$ and $s_{2 z}$ remains unaffected. A mechanism, for example, whereby the sensor-gains change over time is the ageing of the LEDs and phototransistors.

\section{Change in the position ( $x$-coordinate) of a sensor}

In 5.4 , we have seen that the z-position seen e.g. by sensor-2 as a result of the excitation alone can be expressed as

$$
s_{2_{-} z}(t)=x_{s 2} \cdot \tan (\theta(t)) \cong x_{s 2} \cdot \theta(t)
$$

where $\theta(t)$ is the excitation. As long as the excitation (along with the flow) is the same, the motion due to Coriolis forces too will be the same. However, if sensor- 2 moves away from its nominal position (along the x-axis), the z-deflection that it "sees" as a result of excitation will change, as in Figure 5.19.

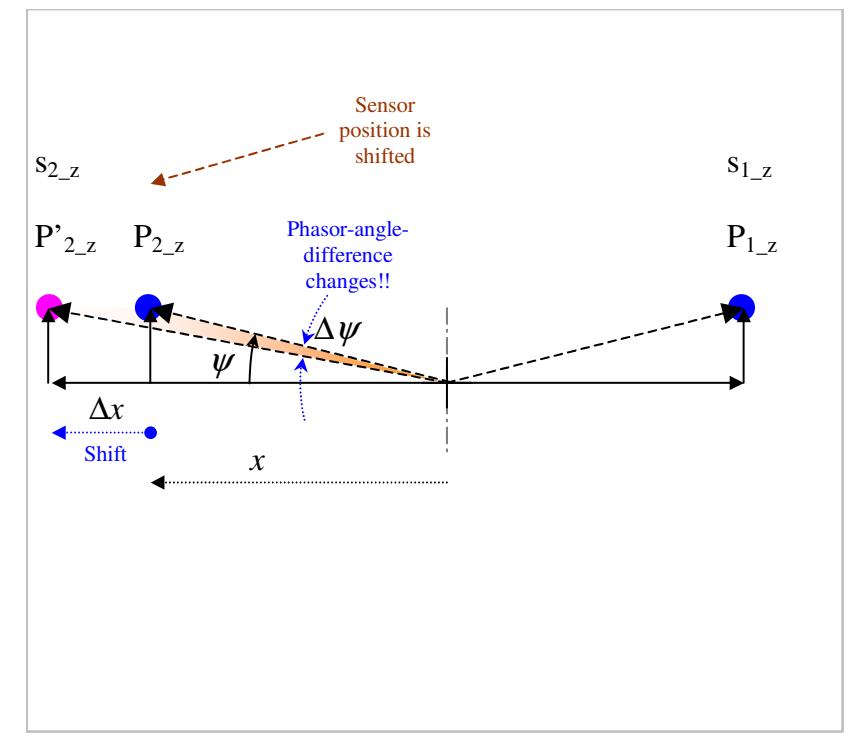

Figure 5.19 Phasor: Change in a sensor's $x$-position changes phasor angle difference 
This will lead to a change in the phasor-angle-difference, $\Delta \psi$, which can be expressed as:

$$
\Delta \psi \cong \psi \cdot \frac{\Delta x}{x}
$$

This error mechanism is unlikely to be active, as the sensors are assembled on a single stiff member (PCB); possible thermal strain is typically $\frac{\Delta x}{x} \cong 11$ [PPM/ ${ }^{\circ} \mathrm{C}$ ] (for FR4 PCB substrate material). No other strain-mechanisms are expected around the sensors, and as such, even with a large temperature-swing $\left(50^{\circ} \mathrm{C}\right)$, a fractional error $(\Delta \psi / \psi)$ of about $0.055 \%$ is anticipated.

\section{Change in the position of the axis-of-rotation of the tube}

Even if the sensors are stationary WRT each other, the environment of the tube (such as temperature gradients, pressure drop, orientation WRT gravity, etc.) can change, causing a sideways-shift in the axis of rotation-oscillation of the tube. In PoP tests, a sideways-shift ranging from $0.5 \mathrm{~mm}$ to several $\mathrm{mm}$ (depending on tube geometry) is seen. We discuss here the effects of such a shift.

The phasor angle of the signal of the first sensor $S_{2}$ is $\psi_{1}$ and that of $S_{2}$ is $\psi_{2}$. Consider, for sake of simplicity, that we represent $\psi_{2}$ as its complementary angle $\psi_{2}{ }^{*}=\pi-\psi_{2}$

As in previous phasors, sensor $S_{1}$ is located at $\mathrm{x}$-coordinate $\mathrm{x}_{\mathrm{s} 1}$ and $\mathrm{S}_{2}$ at $\mathrm{x}_{\mathrm{s} 2}$. The rotation axis is nominally at the origin $(x=0)$

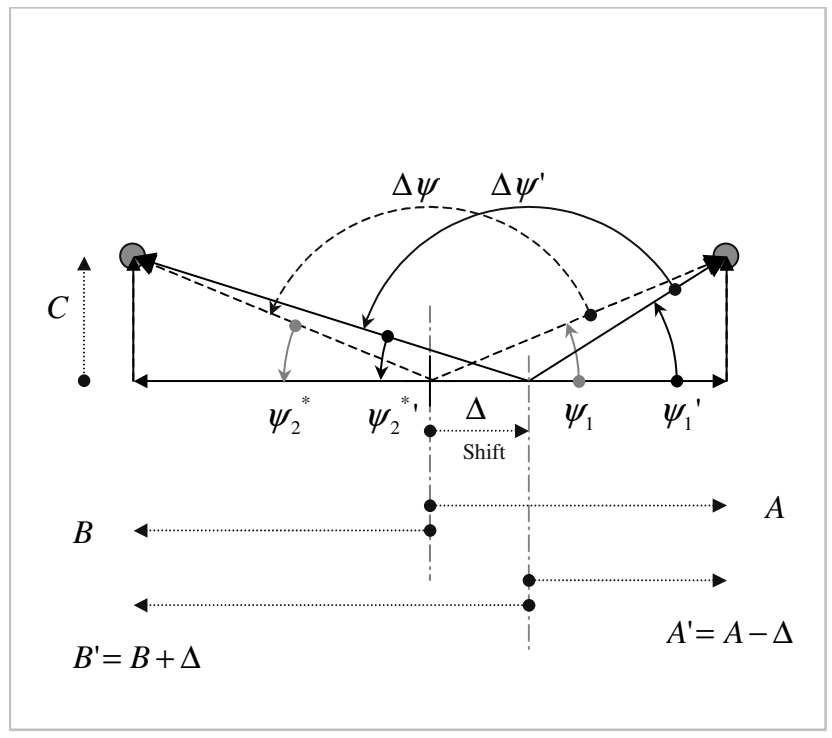

Figure 5.20 Phasor: A shift in the rotation axis changes the phasor-angle-difference

For simplicity, let us say that $\left|x_{s 1}\right|=A$ and $\left|x_{s 2}\right|=B$; initially $A=B=r$ (say)

Now, consider that the rotation axis shifts by a small displacement $\Delta$. In the phasor diagram, shifting the origin of the coordinate axis by the same displacement $\Delta$ can compensate this. However, the coordinates of $\mathrm{S}_{1}$ and $\mathrm{S}_{2}$ change (say, from $\{A, B\}$ to $\left\{A^{\prime}, B^{\prime}\right\}$ ).

$$
A^{\prime}=A-\Delta ; B^{\prime}=B+\Delta
$$

The Coriolis deflection (say $C$ ) is the same (unaffected due to a sideways shift of the rotationaxis). From the phasors, it can be seen that: (for small $\psi$ ) 


$$
\begin{aligned}
& \psi_{1}=\arctan \left(\frac{C}{A}\right) \cong \frac{C}{A} \\
& \psi_{1}^{\prime}=\arctan \left(\frac{C}{A-\delta}\right) \cong \frac{C}{A-\delta}
\end{aligned}
$$

As a result, say that the absolute phasor angle $\psi_{1}$ changes by $d \psi_{1}$.

$$
d \psi_{1} \cong \frac{C}{A-\delta}-\frac{C}{A} \Rightarrow \frac{C \cdot A-C(A-\delta)}{(A-\delta) A} \Rightarrow \frac{C \cdot \delta}{A^{2}-A \cdot \delta} \Rightarrow \frac{C \cdot \delta}{r^{2}-r \cdot \delta}
$$

Along the same lines, it can be shown that

$$
d \psi_{2}^{*} \cong \frac{C}{B+\delta}-\frac{C}{B} \Rightarrow \frac{C \cdot B-C(B+\delta)}{(B+\delta) B} \Rightarrow \frac{-C \cdot \delta}{B^{2}+B \cdot \delta} \Rightarrow \frac{-C \cdot \delta}{r^{2}+r \cdot \delta}
$$

The resultant change in phasor-angle difference will be $\Delta \psi_{12}$, such that:

$$
\begin{gathered}
d \psi_{12}=d \psi_{1}+d \psi_{2}{ }^{*}=\frac{C \cdot \delta}{r^{2}-r \cdot \delta}+\frac{-C \cdot \delta}{r^{2}+r \cdot \delta} \\
=\frac{C \cdot \delta\left(r^{2}+r \cdot \delta\right)-C \cdot \delta\left(r^{2}-r \cdot \delta\right)}{\left(r^{2}-r \cdot \delta\right)\left(r^{2}+r \cdot \delta\right)} \\
=\frac{2 C \cdot r \cdot \delta^{2}}{r^{4}-r^{2} \cdot \delta^{2}} \cong \frac{2 C \cdot r \cdot \delta^{2}}{r^{4}} \\
=\frac{2 C}{r}\left(\frac{\delta}{r}\right)^{2}
\end{gathered}
$$

However, in the initial situation, (see phasor, Figure 5.16) $\frac{2 C}{r}=2 \alpha=\Delta_{1-2}$ corresponds to the phasor-angle change due to Coriolis forces itself. Furthermore, $\delta / r$ is the fractional shift in rotation pole position, where $r$ is the nominal $x$-distance between the rotation axis and any one sensor. A typical value for $r$ (see Table 5-3) is $3.2 \mathrm{~mm}$. For the typical $\delta$ of $0.5 \mathrm{~mm}$, this would lead to a fractional (gain) error $E_{R P}$ in reckoning the amplitudes' ratio of

$$
E_{R P}=\frac{2 C}{r}\left(\frac{\delta}{r}\right)^{2} / \frac{2 C}{r}=\left(\frac{\delta}{r}\right)^{2} \Rightarrow\left(\frac{0.5}{3.2}\right)^{2}=0.023
$$

$$
\text { ( } E_{R P}: \text { Error brought about due to the rotation-axis position shift) }
$$

Such a large error (2.3\%) is not acceptable and needs to be mitigated. This issue is discussed further in [5.4.2].

\subsubsection{Correction for rotation-pole shift using multiple sensors}

It is known that the amplitude accuracy of the (optical) position sensors is poor, while the time-domain response is quite good. For this reason, in $\mathrm{Ch} .4 .4$ we had chosen for the following concept:

Paradigm: In order to make an accurate estimate of wobble (ratio between swing and twist amplitudes), we use purely the time information (direction of phasor arrow) provided by position sensors; we disregard all magnitude information (length of phasor arrow).

Knowing the geometry of the tube and the placement of the position sensors, if we are to ignore all amplitude information from two phasors of an arbitrary (but common) phase 
reference, it is not possible to derive information about the position of a rotation-pole. In order to do this, the use of a third (extra) position sensor is proposed.

Twist excitation axis

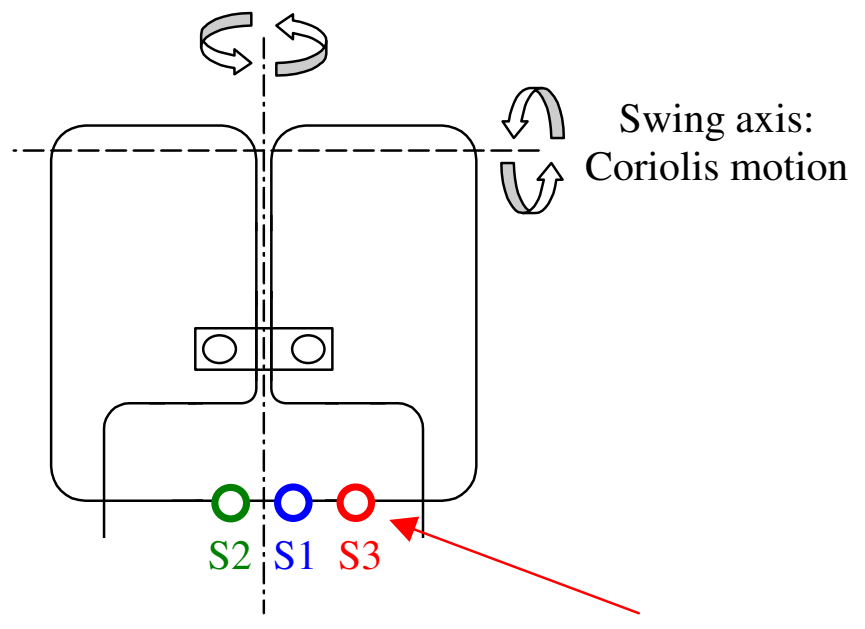

Figure 5.21 Placement of the extra (third) sensor

A third sensor is placed adjacent to the two initial sensors and the position signal read by this sensor is processed in the same manner as the first two sensors. This generates an additional third phasor. For the configuration shown, the phasor would look like Figure 5.22.

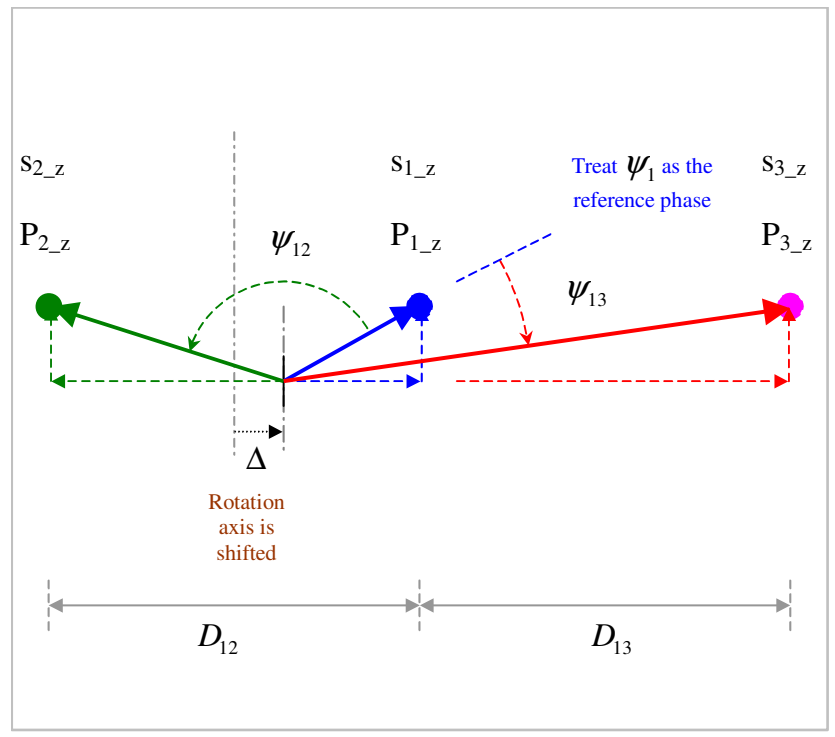

Figure 5.22 Phasors with three sensor signals

To recapitulate we now have accurate information about:

1) The phasor angle difference between the first (which we treat as the arbitrary reference) and the second phasor. We call this angle $\psi_{12}$.

2) The phasor angle difference between the first and the third observation. We call this angle $\psi_{13}$.

3) We also know, from our construction, the quantities $x_{1} x_{2}$ and $x_{3}$, the $\mathrm{x}$-coordinates of the position sensors. These quantities may vary as the pivot-point of rotation 
changes. However, the relative positions of the observers (light sensors) is fixed, and accurately known. In short, we accurately know our intra-sensor spacing $D_{12}$ and $D_{13}$ where $D_{12}=\left|x_{2}-x_{1}\right|$ and $D_{13}=\left|x_{3}-x_{1}\right|$. In fact, $D_{12}=D_{13}$

4) Next, the amplitude information is discarded (see Figure 5.23).

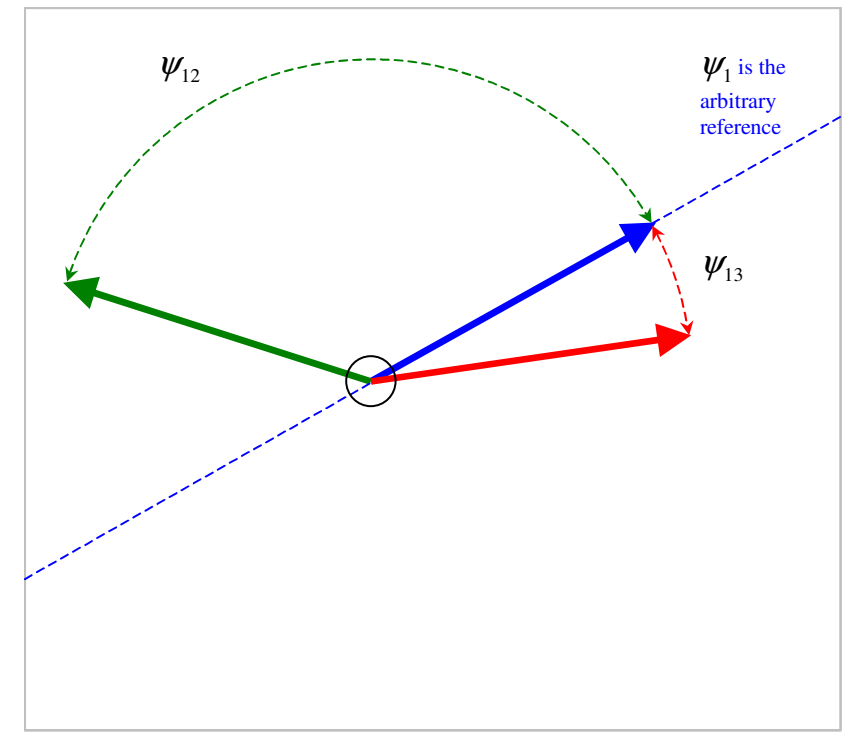

Figure 5.23 Phasors: All amplitude information is discarded

5) We know that the Coriolis force manifests itself as a swing, i.e. an instantaneous sideways deflection that is equal at all points on the sensor tube. We do not know its magnitude, but we do know that it is equal at all three observed locations.

6) Consider the mapping of these phasors on a coordinate plane with an arbitrary scale. (Figure 5.24) The reference phase $\psi_{1}$ is oriented along the $y$-axis for convenience.

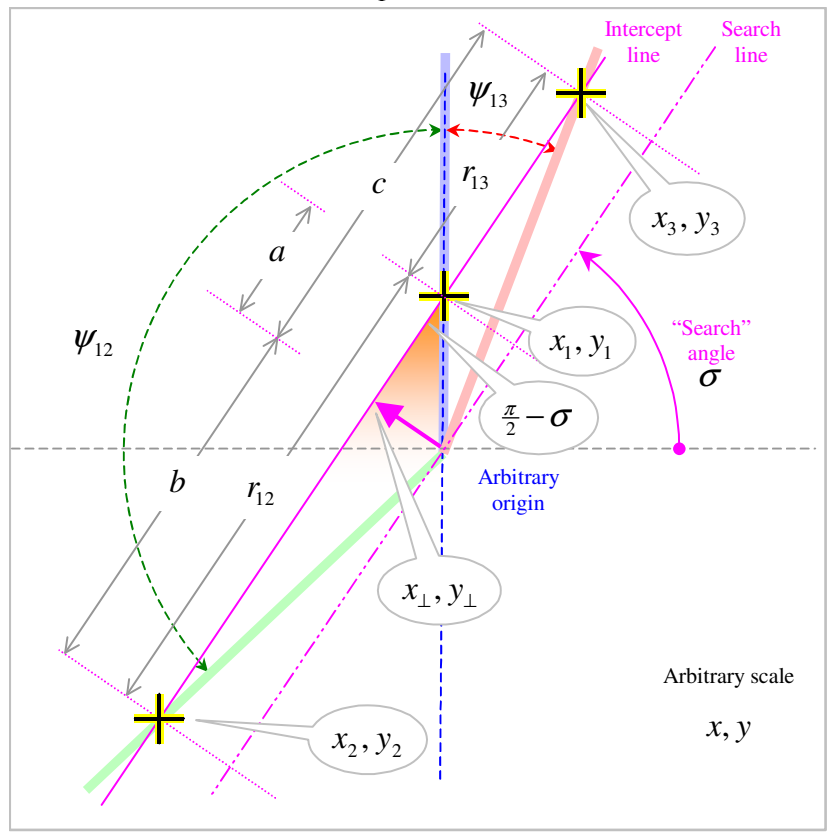

Figure 5.24 Phasors mapped upon an arbitrary scale 
One possible way to determine the amplitudes ratio, independent of the axis-shift, is as follows (refer to Figure 5.24): Consider a "search" line from the origin, with search-angle $\sigma$. Consider also, that an "intercept line" that runs parallel to this search-line at a unit distance, and intersects the sensor- 1 phasor at coordinates $x_{1}, y_{1}$, the sensor- 2 phasor at $x_{2}, y_{2}$ and the sensor-3 phasor at $x_{3}, y_{3}$. The distances between the projections of the various interceptpoints upon the search-line are represented as $a, b$ and $c$ (see Figure 5.24). From geometry,

$$
\begin{aligned}
& a=\tan \sigma \\
& b=\tan \left(\psi_{12}-\sigma\right) \\
& c=\tan \left(\psi_{13}+\sigma\right)
\end{aligned}
$$

Further as $D_{12}=D_{13}$ (see Figure 5.22), relation $r_{12}=r_{13}$ must also hold where

$$
\begin{aligned}
& r_{12}=b+a \\
& r_{13}=c-a
\end{aligned}
$$

Say that a particular search-angle $\sigma^{*}$ satisfies this condition. The rescaled (upon the coordinate axis of Figure 5.24) twist excitation amplitude (referred to sensors 1 and 2) is $r_{12}=b+a$, while the rescaled swing amplitude is unity. The amplitudes' ratio (extent of wobble) is then $1 /(a+b)$, indicative of the mass flow rate.

Interestingly, the shift in the rotation axis can also be determined as

$$
\Delta^{*}=\frac{r_{12}}{2}-a=\frac{b+a}{2}-a=\frac{b-a}{2}
$$

This $\Delta^{*}$ however is on a rescaled coordinate-axis. To scale it back to the inter-sensor spacing, it is multiplied by $S F=D_{12} / r_{12}=D_{12} /(b+a)$, where $D_{12}$ is typically $3.2[\mathrm{~mm}]$. Thus,

$$
\Delta=S F \cdot\left(\Delta^{*}\right)=\frac{D_{12}}{b+a} \cdot\left(\frac{b-a}{2}\right)
$$

\subsubsection{Noise on ratiometric measurement due to noise on position sensor signals}

Here, we consider the effect of electrical and digital (quantization and discretization) noise on the noise on the phasor. Several sources contribute to this:

1. noise on the light emitted in the LED within the position sensor (opto-interrupter)

2. noise in the photocurrent (of the position sensing phototransistor)

3. noise in the resistor that translates current to voltage in the transimpedance amplifier

4. noise in various op-amp devices

5. electrical pickup on the interconnection between the position sensor and the digitizer

6. noise due to quantization (resolution) and discretization (clock jitter) in the digitizer

7. arithmetic and algorithmic noise in the digital processing

These are depicted in the following simplified schematic: 


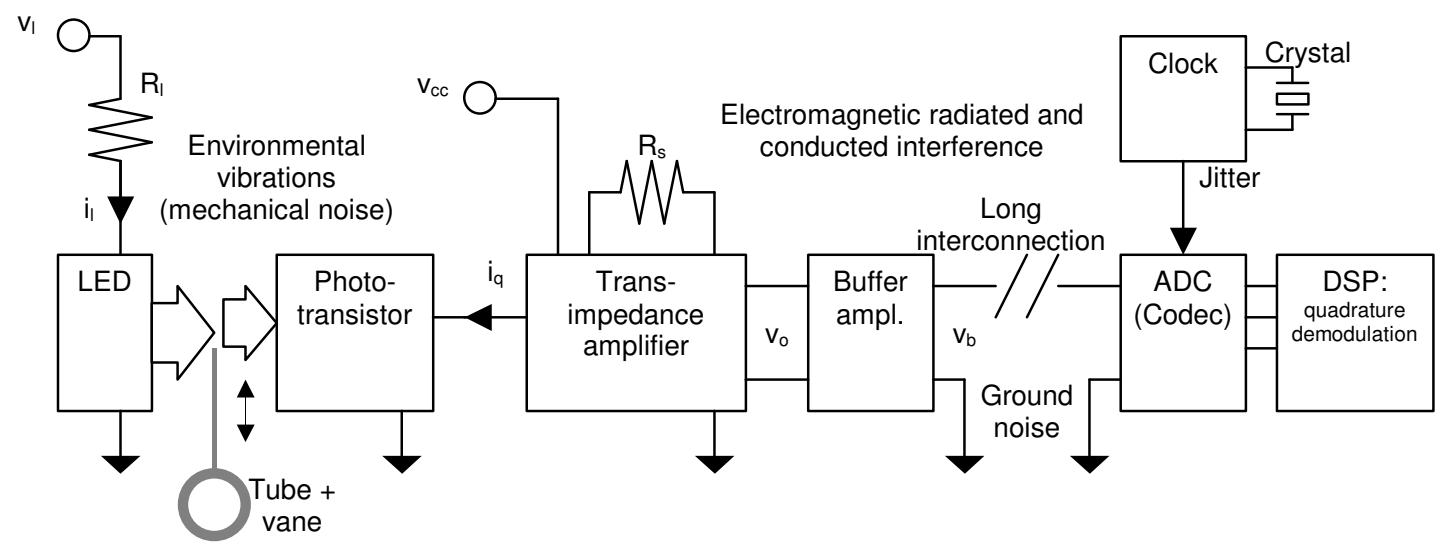

Figure 5.25 Various noise sources in the sensor signal-chain

The noise from each of the sources depicted in Figure 5.25 can be approximately expressed as equivalent flow-noise. The following statements are made, based upon measurements and experiences from PoP tests:

1. The light source (LED) is driven with approximately $20 \mathrm{~mA}$ current. The light is projected upon the phototransistor.

2. The tube and vane create a shadow on the phototransistor that is sinusoidal in time. The frequency of this shadow is the eigenfrequency of the oscillating tube.

3. Mechanical vibrations in the swing direction cause the optical components to move WRT the tube, causing fluctuations in the shadow. These too cause measurement noise.

4. The phototransistor generates photocurrent, around $8 \mathrm{~mA}$ when fully illuminated. This is modulated by the shadow, the mean (quiescent) value being adjusted at about 4 $\mathrm{mA}$. The aperture of the phototransistor is $0.5 \mathrm{~mm}$, giving a "position sensitivity" of $16 \mathrm{~A} / \mathrm{m}$

5. The photocurrent is converted to a voltage signal by means of a (fast, low-noise) transimpedance amplifier. A $200 \Omega$ resistor is used as a sense-resistor, giving a transimpedance of $200 \mathrm{~V} / \mathrm{A}$. The quiescent output voltage is about $800 \mathrm{mV}$, while the position sensitivity is $3200 \mathrm{~V} / \mathrm{m}$.

6. The voltage signal is buffered and transmitted to the ADC - a sigma-delta CODEC (AD1939) with a sampling frequency of $24 \mathrm{kHz}$ and a resolution of 24 bits per 3.2 volts. The A/D conversion noise is dominated by the clock-jitter and not by the converter bits. [datasheet: Analog devices AD1939] The crystal frequency is $25 \mathrm{MHz}$ $(1 / \mathrm{f}=40 \mathrm{~ns})$ with about $5 \%(2 \mathrm{~ns})$ RMS jitter.

7. The sampled signal is processed as 32-bit floating-point data, having 24-bit mantissa. Hence the round-off noise too is negligible, compared to other sources.

8. Steps 1 through 7 occur on 2 or 3 position-sensors. A dual-quadrature-demodulation $\mathrm{w} /$ synchronous detection is applied to these channels, employing a PLL. The effect of this is that the position signal is narrow-band filtered around the tube-eigenfrequency: $f_{\text {eig }} \pm 25 \mathrm{~Hz}$ (The $25 \mathrm{~Hz}$ is a tradeoff between response speed and noise). Antialiasing filters to prevent out-of-band signals affecting measurements are implemented and are assumed to be ideal.

Based upon these assumptions, the following table estimating the equivalent flow-rate noise contribution of each of the various sources may be drawn up: 
Table 5-5 - Noise contribution from various sources

\begin{tabular}{|c|c|c|c|c|}
\hline Source/description & noise & $\begin{array}{l}\text { Equivalent } \\
\text { motion }\end{array}$ & $\begin{array}{l}\text { Equivalent } \\
\text { flow noise }\end{array}$ & Remarks/ Source of information \\
\hline LED current & & $\sim 30 \mathrm{pm}$ & Small & Shot noise; noise on $\mathrm{V}_{\text {led }}$ \\
\hline LED light output & & $?$ & & $1 / \mathrm{f}$ noise \\
\hline Photocurrent & $0.25 \mathrm{nA}$ & $\sim 10 \mathrm{pm}$ & Small & Shot noise: $\sqrt{2 \cdot q_{e} \cdot i \cdot \Delta f}$ (see [5]) \\
\hline $\begin{array}{l}\text { Sense-resistor/ } \\
\text { thermal }\end{array}$ & $12.8 \mathrm{nV}$ & $\sim 3 \mathrm{pm}$ & Small & 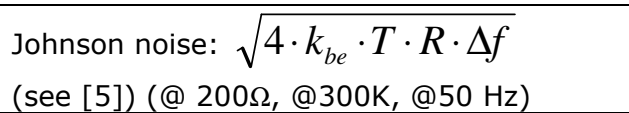 \\
\hline Amplifier i noise & $0.14 \mathrm{pA}$ & - & Small & @ $20 \mathrm{fA} / \mathrm{rtHz}$ \\
\hline Amplifier $v$ noise & $141 \mathrm{nV}$ & $\sim 30 \mathrm{pm}$ & Small & @ $20 \mathrm{nV} / \mathrm{rtHz}$ \\
\hline Buffer v noise & $141 \mathrm{nV}$ & $\sim 30 \mathrm{pm}$ & Small & @ $20 \mathrm{nV} / \mathrm{rtHz}$ \\
\hline $\begin{array}{l}\text { EMI pickup \& } \\
\text { ground noise* }\end{array}$ & & $*$ & & Depends upon implementation! \\
\hline $\begin{array}{l}\text { Converter } \\
\text { resolution }\end{array}$ & $1.8 \mathrm{nV}$ & - & - & Negligible -24 bits resolution \\
\hline Clock jitter & $4 \mathrm{~ns}$ & Note $^{24}$ & & \\
\hline Measured motion & & $5 \mathrm{~nm}$ RMS & $1[\mathrm{~g} / \mathrm{h}]$ & Experimental! \\
\hline Converter noise & $2.37 \mathrm{uV}$ & $0.7 \mathrm{~nm}$ & $0.14[\mathrm{~g} / \mathrm{h}]$ & $\begin{array}{l}\text { In-band portion of }-92 \mathrm{~dB} \text { THD+N @20 kHz, } \\
@ 1.9 \text { VRMS input signal }\end{array}$ \\
\hline
\end{tabular}

It is seen in Table 5-5 that the converter noise is the dominant noise source, and due to the narrow bandwidth, all other noise sources have little contribution. The total component noise in the detector pass-band, calculated in this manner is less than $1 \mathrm{~nm}$. However, as the tube eigenfrequency is quite low (under $1 \mathrm{kHz}$ ), the $1 / \mathrm{f}$ noise $\left(\right.$ flicker $^{25}$ ) of the various components plays a significant role. The EMI pickup and ground-noise will add disturbance directly, and will also add to clock-jitter and thereby increase converter noise. From a pragmatic standpoint, we measure the actual (in-system) converter output is monitored with the input set to zero (volts) with representative wiring. The equivalent motion noise measured in this manner, about $5 \mathrm{~nm}$ (RMS), is 5 - 6 times larger than the expected value.

This could be improved upon, by using pass-band limiting analog filters, a clock signal with lower jitter, or better shielding against radiated/conducted pickups and a stiffer ground. However, as a $5 \mathrm{~nm}$ (RMS) motion corresponds to an acceptably low flow noise, it is not improved any further.

\subsection{Summary}

In this chapter, five critical issues associated with the implementation of a sensitive Coriolis mass-flowmeter have been enumerated and the underlying principles discussed in detail. These are:

1. Shape and form of the sensing tube

2. The use of eigenmodes to minimize actuation power and reduce inter-mode leakage

3. The mechanism behind the generation and the effect of Coriolis forces

4. The concept of ratio measurement based purely on time-domain information

5. The importance - and a novel implementation - of non-contact and galvanically isolated actuation and sensing of the Coriolis tube

\footnotetext{
${ }^{24}$ The clock-jitter will act upon all present frequencies - the jitter/sampling takes place before the quadrature detection. Experimental values are presented here, from actual measurements on a converter with representative wire-traces, having a short-circuited $(v=0)$ sensor. This pragmatic approach also takes into account the conducted and radiated electromagnetic noise in the surroundings of the converter.

${ }^{25}$ The LF noise of the AD8608 op-amp, for example, is stated as $2.3 \mathrm{uV}(\mathrm{p}-\mathrm{p})$ between $0.1 \mathrm{~Hz}$ and $10 \mathrm{~Hz}$.
} 



\section{Evaluation and measurement results}

Several concepts for the design and construction of a Coriolis mass flow rate meter have been chosen in Chapter 4 and worked out in detail in Chapter 5. In this chapter, we evaluate the function of various sub-systems and present measurement results for the instrument as a whole. The measurement results are compared with the targets set in the specifications (listed in Chapter 3).

A typical design cycle consists of stating the requirements (Ch 3 ), making concept choices based on the requirements ( $\mathrm{Ch} 4$ ), working out the concepts, breaking them down into subsystems where possible, (Ch 5), constructing prototypes based upon the concepts, and evaluating these with thorough measurements under varying circumstances (this chapter). This process is done iteratively, with feedback from various intermediate evaluations and measurements driving the concept choice and the detailed design. Evaluation results presented in this chapter may appear to be a conclusion to the selected concepts and constructed prototypes, but they are, from a chronological point-of-view concurrent with the rest of the phases.

Measurement results, on the other hand, presented at the end of this chapter, document the final performance (end-result) of the designed instrument and can typically be presented to specify performance in the 'product flyer' of the instrument, intended for the end user.

\subsection{Evaluation: The tube shape and dimensions}

\subsubsection{Pressure drop across tube}

Prototypes built with several tube diameters are tested with a set of fluids to verify the estimated pressure drops for the various instrument-types.

\begin{tabular}{|l|l|l|l|}
\hline $\begin{array}{l}\text { The measured mass-flow-rate at } 1 \\
\text { bar pressure-drop }[\mathrm{g} / \mathrm{h}] \text { at } 20^{\prime} \mathrm{C}\end{array}$ & Type I & Type II & Type III \\
\hline With air & 14 & 90 & 900 \\
\hline With water & 120 & 1300 & 13000 \\
\hline
\end{tabular}

Furthermore, it is seen that

- The length of the tube is significant in determining the pressure-drop. Bent corners in the tube (bending radius $>>$ tube-diameter) do not add substantially to the drop.

- For some fluids, the transition from laminar flow to turbulent flow occurs at a certain flow-rate that lies within the operational range of the instrument. Artifacts (See Ch. 6.5 , e.g. Figure 6.10) may occur upon the meter response during such a transition. (Example -type II instrument with air at $80 \mathrm{~g} / \mathrm{h}$ or type III instrument with water at $1 \mathrm{e} 4 \mathrm{~g} / \mathrm{h}$ )

Conclusion:

- For the required flow ranges [see Ch 3.1.1] for the types I, II and III meters, (i.e. $2 \mathrm{e} 2,2 \mathrm{e} 3$ and $2 \mathrm{e} 4 \mathrm{~g} / \mathrm{h}$ water and $1 \mathrm{e} 1,1 \mathrm{e} 2$ and $1 \mathrm{e} 3 \mathrm{~g} / \mathrm{h}$ air), the pressure-drop across the meter-tube is somewhat higher than 1 bar ( $1 \mathrm{e} 5 \mathrm{~Pa}$ ). The higher (than calculated) values are perhaps a result of non-smooth inner walls.

\subsubsection{Eigenfrequencies of the meter tube}

For a given tube shape, the dimensions are to be chosen such that:

- The excitation and response eigenfrequencies are separated at least by a factor 1.5 
- Each of the frequencies is to be away $(10 \mathrm{~Hz})$ from odd multiples of mains-frequencies

- Other eigenfrequencies of unused eigenmodes should not lie in the neighborhood of these two eigenfrequencies (excitation and response)

The basic form of the tube is the folded rectangular window shape as outlined in $\mathrm{Ch}$. 5.1.3. In this form, the parameters that can be manipulated are:

1) The height of the tube-window ' $\mathrm{H}^{\prime}$

2) The width of the tube-window ' $W$ '

3) The height of the joined central inlet and outlet tubes that form a torsion spring ' $S$ '

4) The amount of solder material added between the central tubes ' $\mathrm{J}$ '

5) The gap between the central tubes ' $G$ '

Additionally, the tube diameter, the tube wallthickness and the bending radii may also be manipulated, but this is not advisable, in order to stay with standard tube cross-section and bending tools.

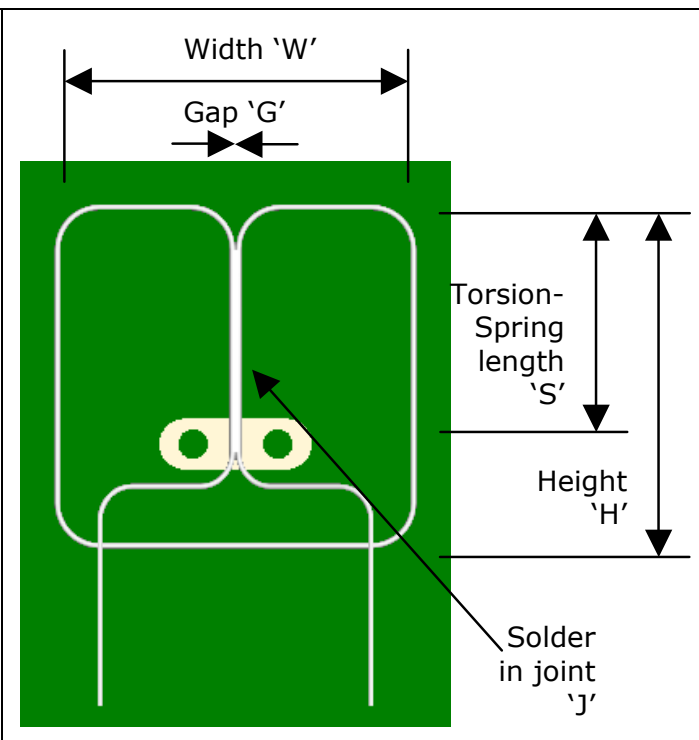

Various tube eigenmodes and their eigenfrequencies for an empty (air-filled) tube

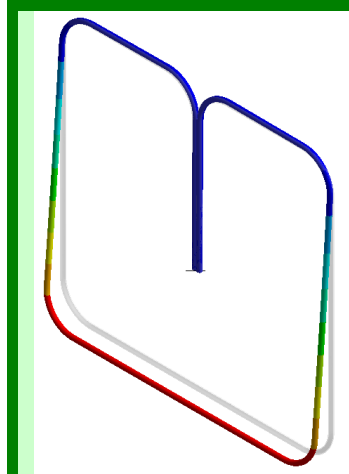

Mode 1 - Swing

Approx. $0.58 \cdot f_{\text {eig }}$

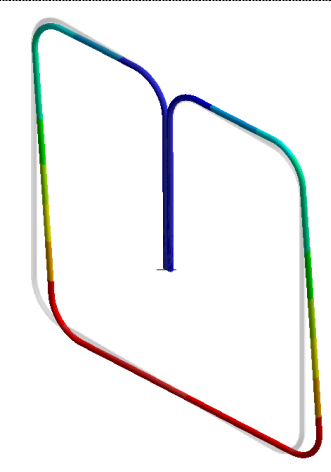

Wave (in-plane)

Approx. $0.82 \cdot f_{\text {eig }}$

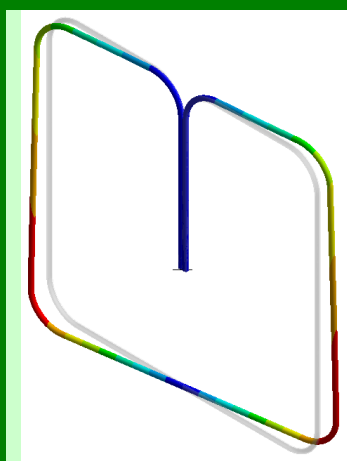

Mode 2 - Twist

$f_{\text {eig }}$

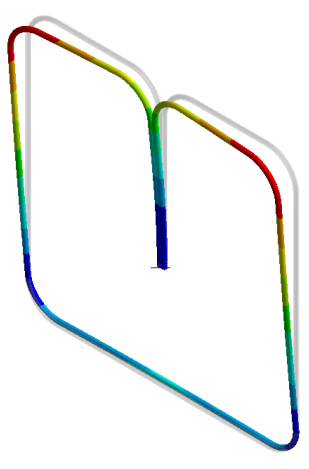

Sway

Approx. $2.05 \cdot f_{\text {eig }}$

The evaluation of the various frequencies and the values of the two frequencies-of-interest is carried out by finite-element simulation and prototype-measurements. The eigenfrequencies are designed in the following manner:

1) FEM simulation:

Assuming some initial dimensions, various eigenfrequencies are determined. Approximate frequency placement, separation between various eigenfrequencies and the factor-1.5 separation between modes 1 and 2 can be done here.

\section{Reference simulation - Type II instrument tube}

\begin{tabular}{|c|c|c|c|c|c|c|c|c|}
\hline \multicolumn{1}{|c|}{ Dimensions } \\
\hline $\begin{array}{c}\mathrm{H} \\
{[\mathrm{mm}]}\end{array}$ & $\mathrm{W}[\mathrm{mm}]$ & $\mathrm{S}[\mathrm{mm}]$ & $\mathrm{G}[\mathrm{mm}]$ & $\mathrm{J}\left[\mathrm{mm}^{3}\right]$ & Swing & Wave & Twist & Sway \\
\hline$H$ & $1.024 \cdot H$ & $0.610 \cdot H$ & $<$ small $>$ & $0.05 \cdot S \cdot G^{2}$ & $0.58 \cdot f_{\text {eig }}$ & $0.82 \cdot f_{\text {eig }}$ & $f_{\text {eig }}$ & $2.05 \cdot f_{\text {eig }}$ \\
\hline
\end{tabular}


2) FEM - Fractional ${ }^{26}$ effect of individual parameters:

Intuitively, changing the tube width will contribute more to the twist moment-of-inertia and hence the mode-2 eigenfrequency. Changing the height will affect the swing moment of inertia, and hence the mode-1 eigenfrequency. In the FEM simulation, the fractional effect of changing each of the dimensions on the eigenfrequencies can also be determined.

Fractional eigenfrequency change per fractional dimension-change (dimensionless)

Eigenfrequencies The dimensions varied:

\begin{tabular}{|l|c|c|c|c|c|} 
& H & W & S & G & J \\
\hline Swing & -1.4 & -0.70 & -0.02 & -0.03 & 0.01 \\
\hline Twist (excit.) & -0.65 & -1.18 & -0.33 & -0.30 & 0.08 \\
\hline
\end{tabular}

3) Prototype measurement:

Due to differences between actual and assumed material properties (density and E-modulus from material datasheets) and simplifying assumptions made in the FEM model, the actual eigenfrequencies are somewhat different than those in the model. However, the simulated fractional variation of eigenfrequencies with tube dimensions can be used as a correction upon the measured frequencies. If the actual dimensions of the prototype were to be changed fractionally (as predicted by the simulations) the resulting (corrected) tube-shape will closely yield the desired eigenfrequencies.

It is seen that due to manufacturing tolerances, especially in the gap ' $G$ ' and the amount of solder added to the joint ' $\mathrm{J}$ ', a piece-to-variation of about $0.5 \%$ is seen in the Mode 1 and Mode 2 eigenfrequencies of fabricated tubes. Finer tuning is pointless.

\subsection{Evaluation: the oscillation properties}

\subsubsection{The $Q$ factor and its relation to mass flow and the environment}

Damping, driven by the (angular) velocity of the tube, generates an effort proportional to the instantaneous (angular) velocity, and thus in-phase with the Coriolis force. For the sake of simplification, let us say that all damping occurs as a lumped effect at a pair of points (antiparallel and more-or-less symmetric) in the bottom section of the Coriolis meter tube. As the moment-arm of the "Coriolis motion" is largest here, any Coriolis-like forces at this location will cause an error in the measurement of the actual Coriolis force. A lower Q-factor is indicative of higher damping; to have an acceptably small Coriolis-like force due to this damping, higher symmetry of construction of the tube will be necessary.

\section{Estimation of Q-factor of a tube:}

As per one of the procedures outlined in Ch. 4.1.1, the Q-factor is determined by measuring the decay time. The Q-factor is also seen to have a fixed relation with the actuation current; however the actuation current is not a reliable indicator for the Q-factor because:

1) The gains of various sensors and transducers are not constant across instruments. One instrument may need more actuation current simply because the biasing magnets are weaker, or because the position-sensing opto-sensors are less sensitive.

2) The gains are not constant in time. Instruments gains are influenced by warming-up and ageing. (An increasing current consumption does not necessarily suggest a dropping Q-factor.)

The decay-time technique is not dependent on the sensor gain - and hence suitable here.

\section{Estimation of damping asymmetry and actuator asymmetry:}

It has been seen in proof-of-principle tests that wind damping is responsible for about half the dissipation in a Coriolis tube. This can be inferred from the fact that if the air surrounding the tube is evacuated, (pressure reduced to 0.03 bara in experiments) the Q-factor approximately doubles. This behavior can be used to test the combined net asymmetry of the actuator and

${ }^{26}$ Fractional as in $\frac{\Delta f}{f} / \frac{\Delta l}{l}$ 
(wind) damping. In vacuum, the actuation-current-consumption (for the same amplitude of oscillation) drops to half its initial value. It may be argued that in this new situation,

1) The wind damping (and hence any tube asymmetry effect of it) is zero

2) The actuation effort is about half its normal magnitude- any offset due to asymmetric actuation is thus also halved.

These two aspects are evaluated for a set of instruments by evacuating the air around the oscillating tubes. The combined effect of actuator asymmetry and asymmetric wind-damping causes an offset-change of less than $0.1(\mathrm{~g} / \mathrm{h})$.

\subsubsection{Actuation - power requirement and relation to the Q-factor}

We do not expect the power requirements for multiple instruments with the same Q-factor to be identical - this difference is due to different component-gains; it is not essential to calibrate these component gains for proper operation of the instrument, as the measurement is based on ratios. This means that the actual tube oscillation amplitude in each instrument in a set of instruments can be slightly different. This means that each instrument will consume a slightly different power for actuation purposes. Furthermore, a Q-factor change will cause an inversely proportional change in the required actuation power.

A way to temporarily increase the Q-factor of a tube is to evacuate it. Similarly, a way to temporarily decrease the Q-factor of a tube is to set the instrument on (dissipative) damping suspension.

The estimated power requirement:

In Ch. 5.2.2, we have estimated the current required to drive the Coriolis tube in steady-state oscillations as about $13.2 \mathrm{~mA}$. Apart from he $\mathrm{i}^{2} \mathrm{R}$ losses in the tube, the back-EMF generated in the tube is:

$$
e_{b}=2 \cdot B \cdot l \cdot \dot{\theta} \cdot r \Rightarrow 2 \cdot \underbrace{4.0 e-1}_{B} \cdot \underbrace{8.0 e-3}_{l} \cdot \underbrace{28}_{\dot{\theta}} \cdot \underbrace{12 e-3}_{r}=2.3[\mathrm{mV}]
$$

thus,

$$
P_{\text {peak }}=e_{b} \cdot i \Rightarrow 2.3 e-3 \cdot 13.2 e-3 \Rightarrow P_{\text {peak }}=29[\mu \mathrm{W}]
$$

(A) - The excitation

This can also be calculated in a different way (as a check):

The instantaneous power $P$ dissipated in a damped body with damping $d$ and instantaneous angular velocity $\dot{\theta}$ is:

$$
P=\mathrm{T} \cdot \dot{\theta}=(d \cdot \dot{\theta}) \cdot \dot{\theta}
$$

Where the damping torque $\mathrm{T}$ is the damping $d$ times the instantaneous angular velocity $\dot{\theta}$.

From 4.1.1, we have:

$$
Q=\frac{1}{d} \sqrt{m \cdot k}=\frac{1}{d} \cdot \frac{k}{\omega_{e i g}}
$$

For the type-II instrument for example, for excitation (twist) motion, $k=7.1 e-2[N \cdot \mathrm{m} / \mathrm{rad}]$, and $Q \approx 2000$ (measured). 
From this we can estimate the damping:

$$
d \approx 3.3 e-8[N \cdot \mathrm{m} /(\mathrm{rad} / \mathrm{s})]
$$

and

$$
P_{\text {peak }} \approx \underbrace{3.3 e-8}_{\text {damping }} \cdot(\underbrace{28}_{\text {peak angular rate }})^{2} \Rightarrow P_{\text {peak }} \approx 29[\mu W]
$$

(B) - The dissipation

That $A$ (Eq. 6.2) and B (Eq. 6.6) are identical is not remarkable - they both originate from the same set of data - Q-factor, stiffness, eigenfrequency, deflection etc. A good point for validation, however, is the match between the expected current consumption and the actual current consumption in a prototype (see schematic - Figure 6.1).

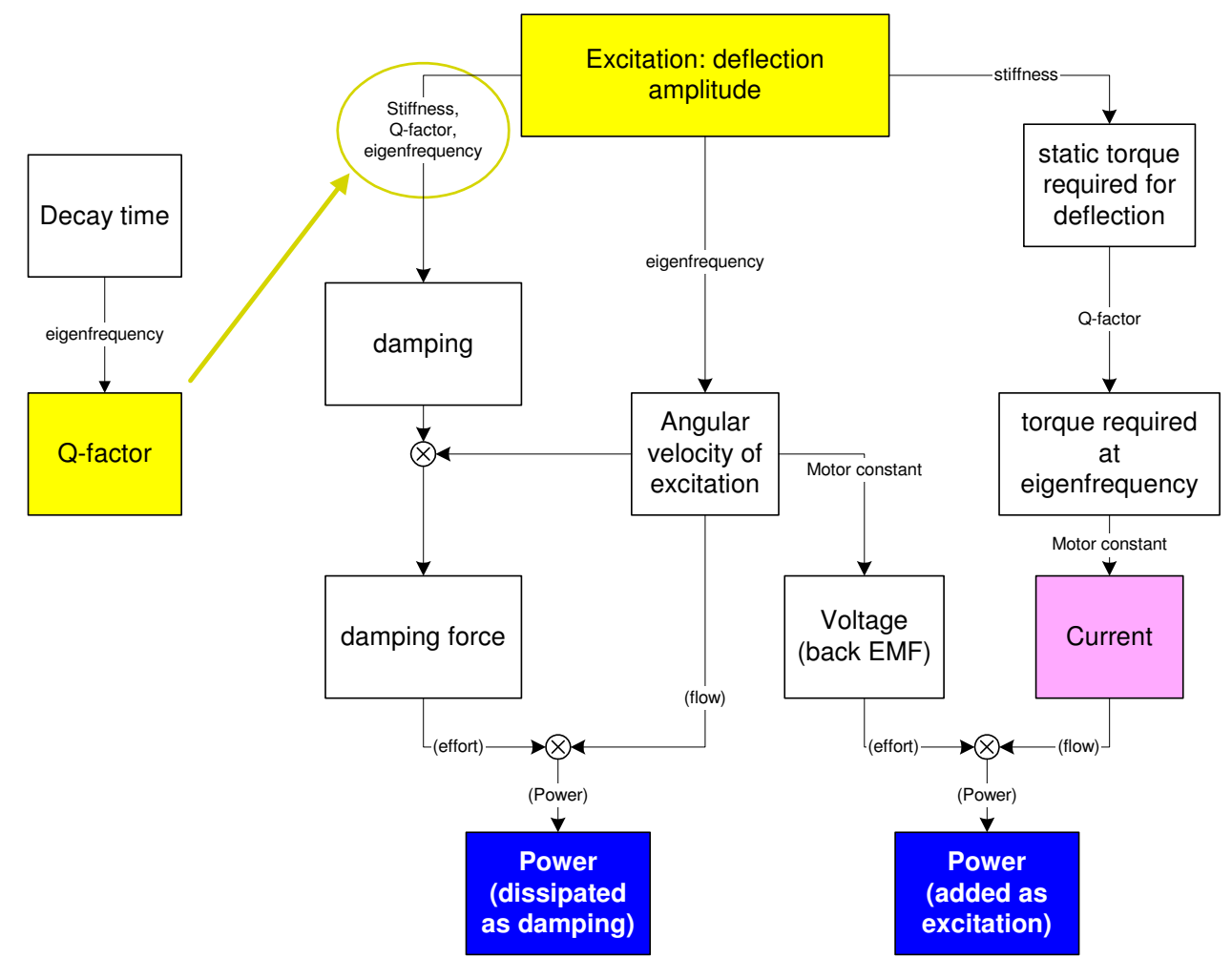

Figure 6.1 Schematic to compare power input and power dissipation

Measurement method:

In an instrument operating at steady-state, a single turn of a conductor is placed as a secondary winding in the current-inducing transformers. From the potential across this oneturn secondary, we infer the volts-per-turn present in the transformers. The ohmic resistance of the (single turn) Coriolis tube is known (from calculations as well as ohm-meter measurements) to be 0.63 [ohm]. At steady state, the voltage (induced by the transformer) will be equal to the phasor sum of the back-EMF and the $i \cdot R$ drop. Thus, upon measuring the transformer volts-per-turn, and approximately knowing the back-EMF, we can calculate the actual current in the tube. A second possible method is to use a clamp-on current meter to directly measure the current in the tube; this method is inconvenient due to space constraints inside the instrument 
Table 6-1 Measurements:

\begin{tabular}{|l|l|l|}
\hline Instrument & Type II & \\
\hline Excitation angular rate & 28 & {$[\mathrm{rad} / \mathrm{s}]$} \\
\hline Estimated back EMF & $2.3 \mathrm{e}-3$ & {$[\mathrm{~V}]($ peak) $(\mathrm{u}=\mathrm{L} \cdot \mathrm{B} \times \mathrm{V})$} \\
\hline Measured volts-per-turn induced in the tube & $5.6 \mathrm{e}-3 \times 2=11.2 \mathrm{e}-3$ & {$[\mathrm{~V}]$ (peak, measured) } \\
\hline i.R drop & $8.9 \mathrm{e}-3$ & {$[\mathrm{~V}]$ (peak) $(0.63 \mathrm{ohm})$} \\
\hline Excitation current & $14.1 \mathrm{e}-3$ & {$[\mathrm{~A}]($ peak) } \\
\hline Excitation power & $32.4 \mathrm{e}-6$ \\
\hline Dissipation power (calc. From Q-factor) & $29 \mathrm{e}-6$ & {$[\mathrm{~W}]$ (peak) } \\
\hline
\end{tabular}

Measurements confirm that the actuation power is indeed equal to the estimated dissipation. The approximate value of the flux-density in the actuator, and the sensitivity of the tubeposition sensor account for the slight mismatch in the expected and measured values. In practice, at sustained (constant amplitude) oscillations, the two will be exactly equal.

\subsubsection{Effects of a non-ideal actuator}

The Coriolis tube is brought into oscillations by means of a Lorentz actuator. Asymmetry in this actuator (magnet-yoke) will directly add offset to the Coriolis measurement as discussed in 5.2.3. Here we evaluate the error introduced as a function of the asymmetry.

By varying the Q-factor of the oscillating Coriolis tube, and then observing the offset on the flow measurement, it is possible to detect asymmetry in the actuator. One way to change the Q-factor is discussed in Ch. 6.2.1 (vacuum), however, the tube symmetry also plays a role in case of evacuation. In another approach, changing the Q-factor by adding dampers to the instrument-suspension will change the Q-factor without affecting the air damping, but the dampers will introduce their own motion, which can be misinterpreted as flow. In-system measurement of the actuator asymmetry can then only be done with a "known symmetric" Coriolis tube, where, in case of evacuation, all errors can be attributed to actuator asymmetry.

It is also possible to intentionally disturb the actuator symmetry (for example by adding ferromagnetic "shims" to increase the pole-surface area and thus reduce the flux density in one of the air-gaps. By doing this, it is possible to verify the predicted (in 5.2.3) effect of actuator asymmetry. 


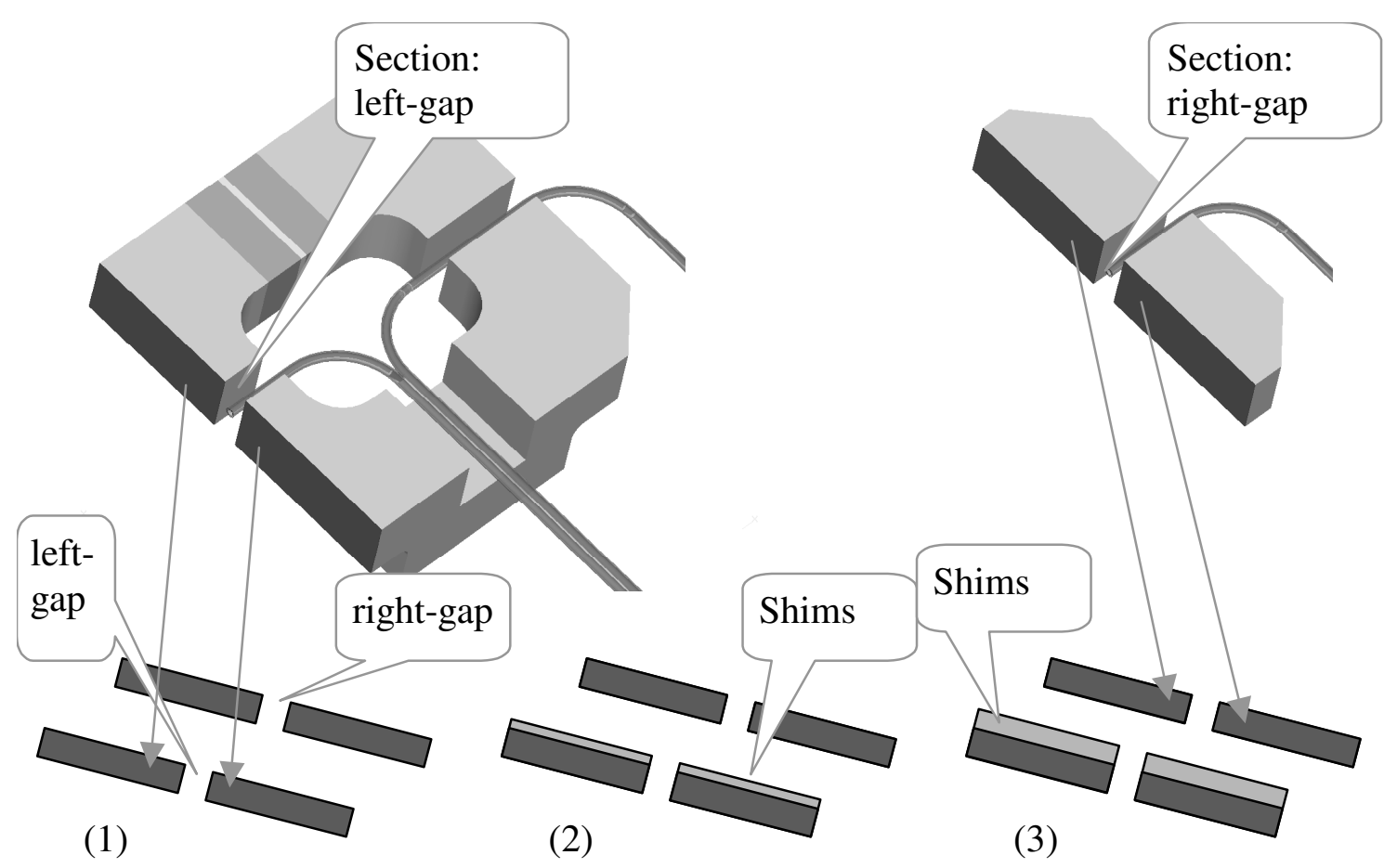

Figure 6.2 Simulating actuator asymmetry with ferromagnetic shims

The figure Figure 6.2 illustrates the idea - (1) shows the normal symmetric configuration. In (2), shims are added to the left gap thereby increasing the pole area. This is further aggravated in (3) by thicker shims. The effect on offset upon the measured flow is measured for each situation.

Table 6-2 Asymmetric actuator: measurements

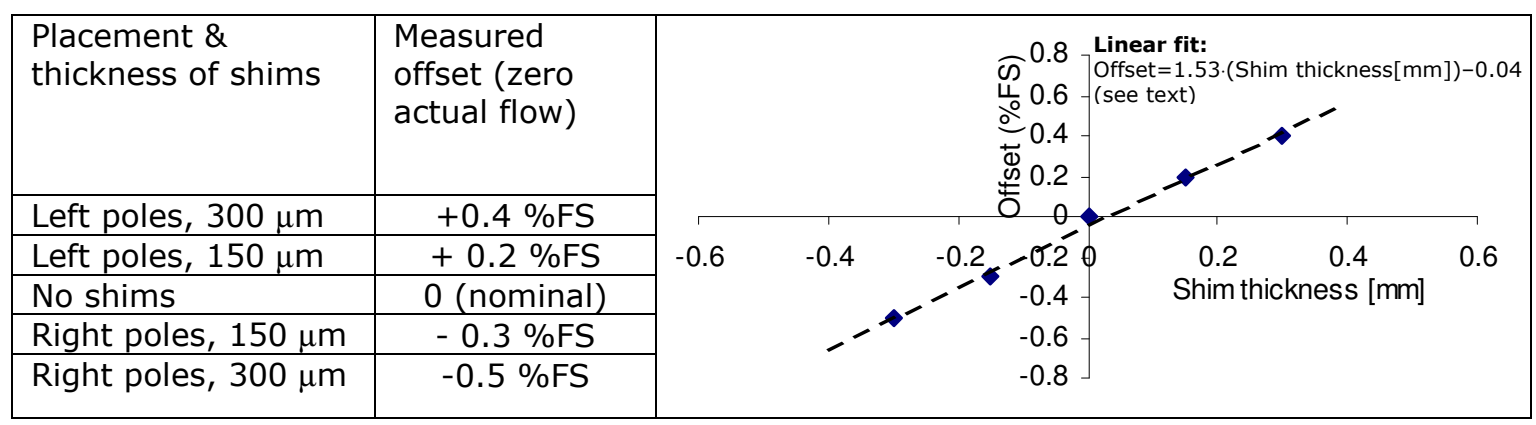

The measured error brought about by making the stator yoke asymmetric is about $0.1 \% \mathrm{FS}$ per $60 \mu \mathrm{m}$ thickness-mismatch - somewhat less than the anticipated $0.1 \%$ FS per $35 \mu \mathrm{m}$ mismatch. This can be due to the proximity of the response (swing) mode's rotation axis to the point of actuation. The order of magnitude of the introduced error does indeed match the prediction made in Ch. 5.2.3.

\subsubsection{Temperature and tube eigenfrequencies}

The variation of the tube eigenfrequency as a function of temperature is seen to follow the same trend as the tube-torsion experiment described in 5.2.5. The fractional change in eigenfrequency per change in temperature is (as a linear approximation) seen to be 


$$
k_{f t(\text { lin })}=\frac{\Delta f_{\text {eig }} / f_{\text {nom }}}{\Delta T} \Rightarrow-2.3 e-4>k_{f t(\text { lin })}>-2.5 e-4[\mathrm{~Hz} /(\mathrm{Hz} \cdot \mathrm{K})]
$$

for several instruments, with the nominal eigenfrequency f as diverse as $30 \mathrm{~Hz}$ and $400 \mathrm{~Hz}$

If stiffness is assumed to change linearly WRT temperature (and not the eigenfrequency), then a quadratic coefficient may be used to express the relation between the fractional change in eigenfrequency and the change in temperature - the square of the eigenfrequency varies as the stiffness.

$$
k_{f^{2} t(\text { quad })}=\frac{\Delta\left(f_{\text {eig }}{ }^{2}\right) / f_{\text {nom }}{ }^{2}}{\Delta T} \approx-4.8 e-4\left[\mathrm{~K}^{-1}\right]
$$

As the fractional change in eigenfrequency due to temperature over the entire temperature range of the instrument is expected to be less than $2 \%$, the linear approximation is used in the actual instrument (seen in section 6.5). As the proportionality is a purely a material property, it stays constant across instruments ( $+/-5 \%$ is observed) as long as a tube of the same alloy is used.

\subsubsection{Medium density and the tube eigenfrequencies}

Eigenfrequencies with various fluids are measured for a batch of instruments.

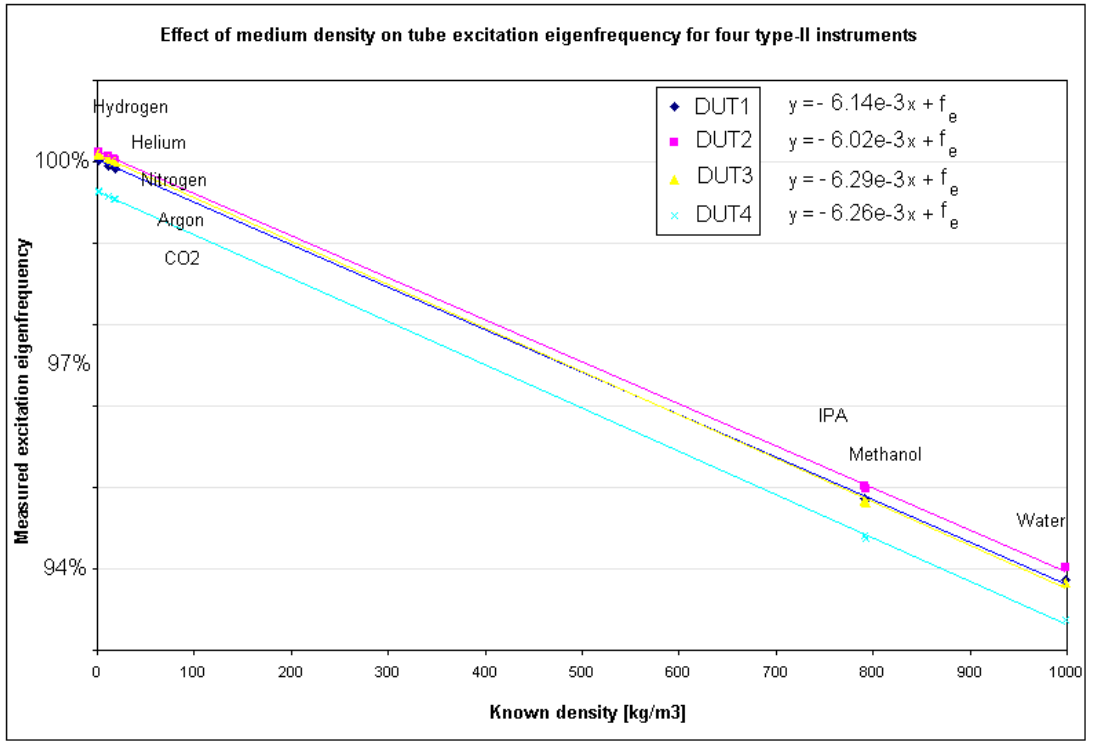

Figure 6.3 Instrument eigenfrequencies with various fluids (media densities)

Due to piece-to-piece variation, the excitation eigenfrequencies of instruments, both dry and wet (filled with water at a known temperature), are seen to differ by a few \%. A "two point" calibration is hence carried out for all instruments - the excitation frequency of the tube filled with uncompressed air subsequently filled with water is measured. These proportionality constants are determined, per instrument, for the measured eigenfrequency values and are thereafter used in determination of density.

Suppose that the tube and the fluid contribute to the inertia of the tube as a whole. Suppose that the volume of fluid in the tube stays constant (irrespective of fluid density). The inertia is expected to affect the inverse squared eigenfrequency: 


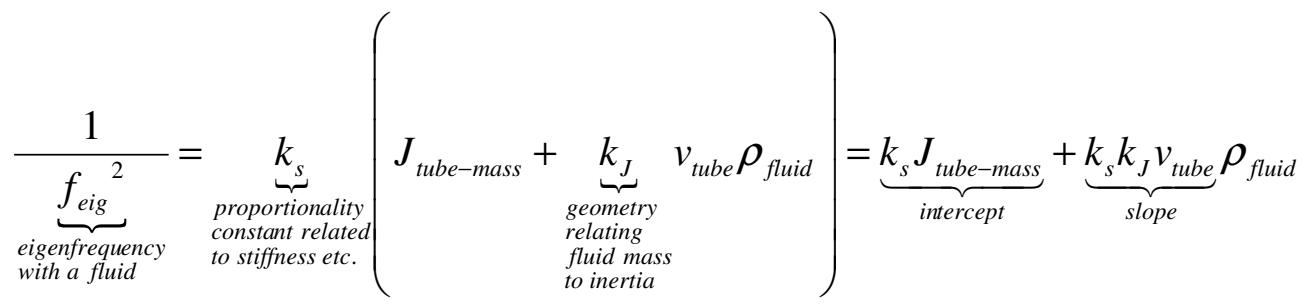

The measured data are used to determine the slope \& intercept in this form:

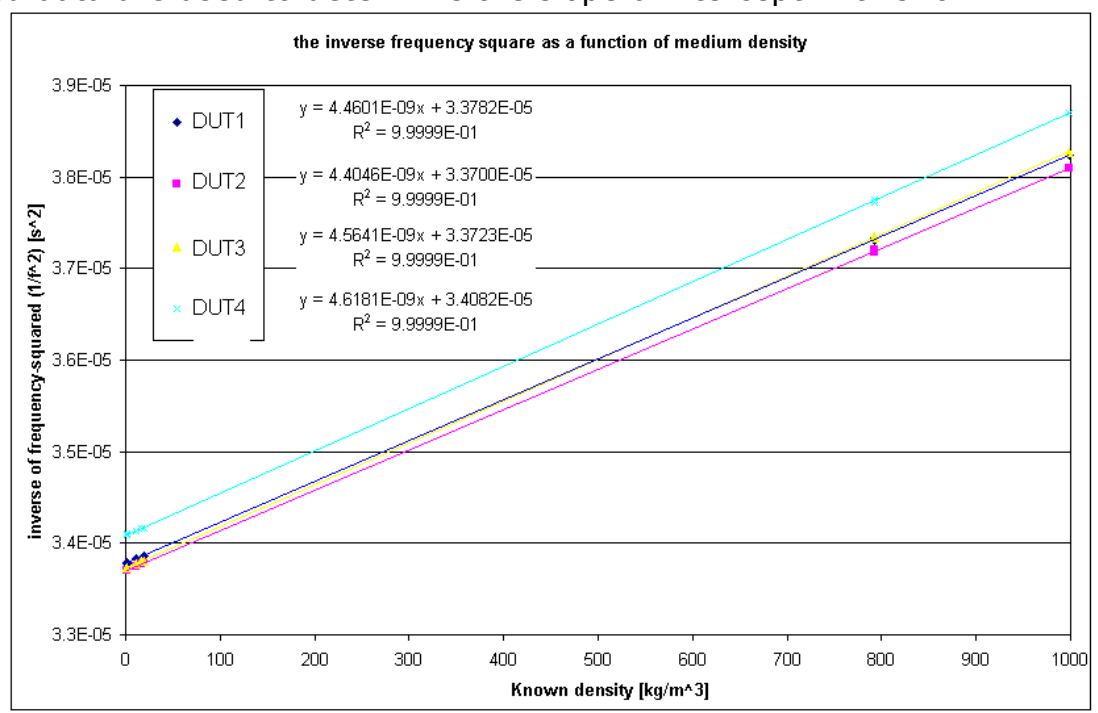

Figure 6.4 Densities mapped to the inverse-squared eigenfrequencies

The slope (proportionality constant) is not normalized to the nominal-frequency (as is done with temperature) - the reason is that for a different tube-geometry (for each tube) the proportionality is substantially different, and hence independently measured. From the slope \& intercept values measured from the two-point calibration, the density of an unknown fluid medium may be calculated from the tube eigenfrequency.

We can conclude from the evaluation that:

- A relative eigenfrequency change of $\sim 6 \%$ is seen between air and water $\left(\Delta \rho \approx 1000 \mathrm{~kg} / \mathrm{m}^{3}\right)$

- Per-instrument-two-point-calibration is necessary for density calculation Further, it is observed that the medium-density has no influence on Q-factor and excitation power requirement.

\subsection{Evaluation: the Coriolis effect}

\subsubsection{Density-related variation in the instrument sensitivity}

In chapter 5.3, a relation between medium-density and the sensitivity (amplitudes' ratio per mass flow) is predicted. This is evaluated here. In the production-instrument, a correction based on the prediction shall be applied.

The DUT is configured as a controller, knowing the approximate sensitivity. The output of the DUT is monitored against the reading of a reference instrument (weigh-scales for liquids and piston-prover for gases) differentiated in real-time. With this method, the approximate sensitivity can be fine-tuned, and small deviations in the sensitivity can be observed in detail, by measuring over a longer time. 
For the type-II instrument, the non-linearity has the same order of magnitude as the accuracy of the reference instrument, and so is neglected. For each sample instrument, normalizing the sensitivity using water as the reference medium (sensitivity with water is set to 1.000), the sensitivity of the instrument to other media is measured. The following results are seen:

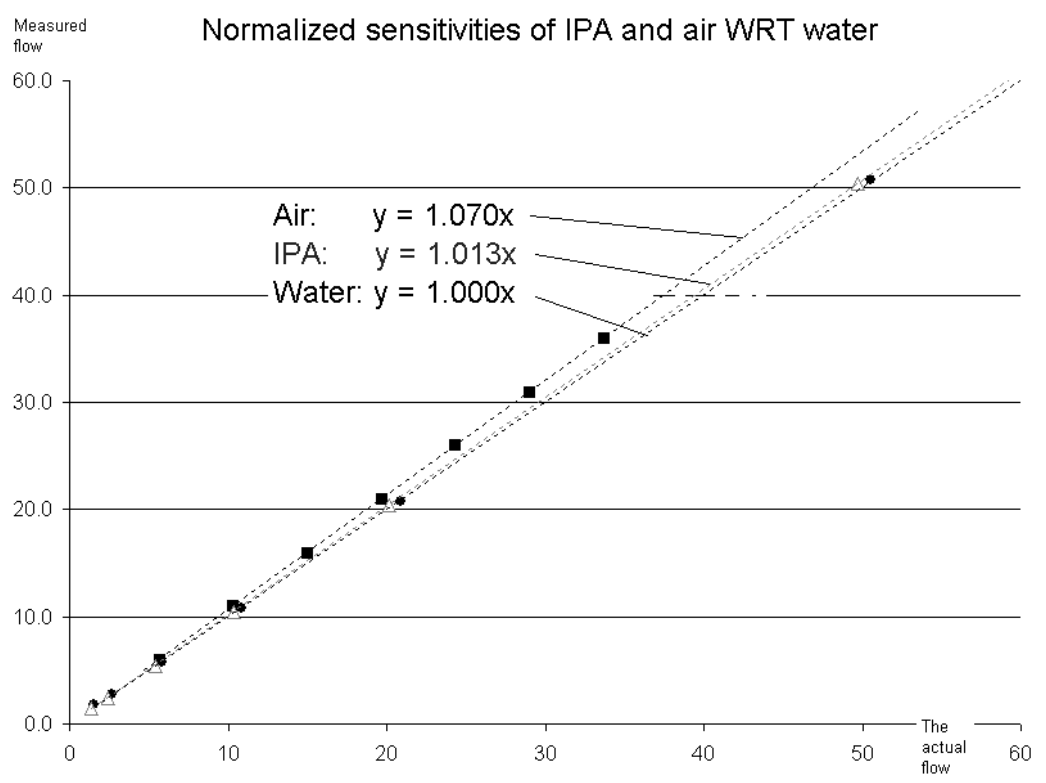

Figure 6.5 Observed sensitivity variation with medium density

The sensitivity of the instrument may be compared to the prediction from 5.3.1:

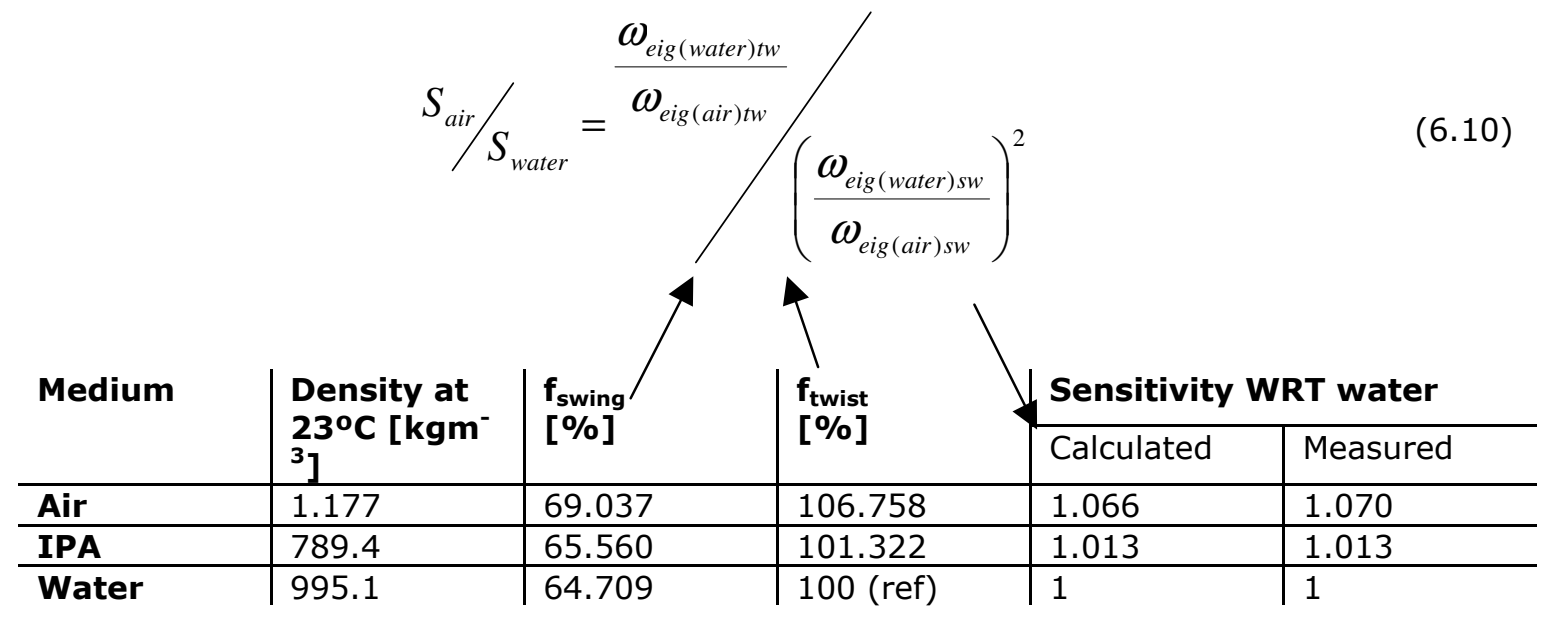

The measured change in instrument sensitivity matches the predicted change - the mechanism proposed in 5.3 is dominant. However, use of Eq. 6.10 poses a practical problem it is difficult to directly measure the swing eigenfrequencies in a production-instrument. An approximation is used instead.

As the Coriolis tube is reasonably homogenous over its length, when a fluid is added to the tube the relative change in swing MI is almost the same as the relative change in the twist MI. Hence, 


$$
\frac{\omega_{\text {eig }(\text { water }) t w}}{\omega_{\text {eig(air }) t w}} \approx \frac{\omega_{\text {eig }(\text { water }) s w}}{\omega_{\text {eig(air }) s w}}
$$

Substituting this in the equation 6.10,

$$
S_{\text {air }} / S_{\text {water }} \approx \frac{\omega_{\text {eig (air)tw }}}{\omega_{\text {eig (water }) t w}}
$$

[The sensitivity varies directly as the tube eigenfrequency (an approximation);

Thus: Lighter medium: lower inertia: higher eigenfrequency: higher sensitivity]

This approximation is used in the production instruments, and is to be found in the measurements in Ch. 6.5.

\subsubsection{Temperature-related variation in the instrument sensitivity}

A theory predicting the variation in instrument sensitivity as a function of the ambient temperature (due to its effects on stiffness) is presented in 5.3.2. The prediction is that the sensitivity is inversely proportional to the eigenfrequency:

$$
\frac{S_{\text {hot }}}{S_{\text {cold }}} \propto \frac{\omega_{\text {eig (cold) }}}{\omega_{\text {eig }(h o t)}}
$$

[Varies inversely as the eigenfrequency;

Hotter tube: lower stiffness: lower eigenfrequency: higher sensitivity]

To evaluate the prediction, the following experiment is used:

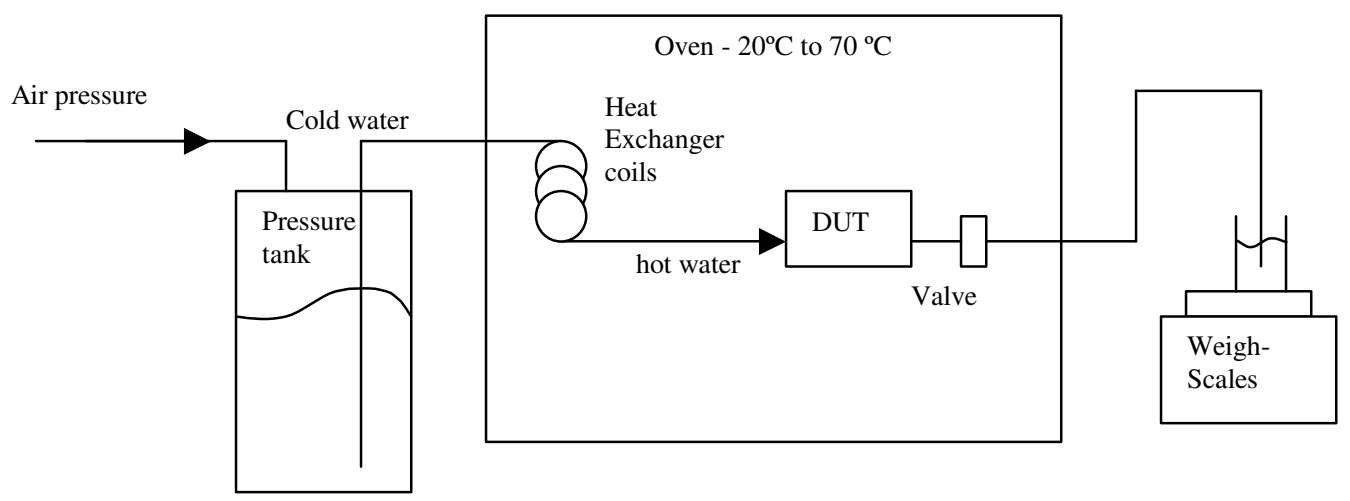

Figure 6.6 Test set-up for measuring sensitivity at various temperatures

The device under test is placed in an oven, maintained at a specified temperature. The device is instructed to, by means of a valve, control MFR at a certain rate. A pressurized flow of water (preheated to the oven temperature by means of a heat exchanger) is made available to the DUT. Under equilibrium conditions (to ensure no change in internal fluid storage), the difference in the weigh-scale reading over a time interval is noted. The ratio of the product of the DUT setting and time interval to the weigh-scale measurement is the relative sensitivity of the DUT. At the elevated temperature, the density of the water is reduces significantly. The 
density of water known from reference tables is used with the density-compensation described in 6.2.5 and 6.3.1 to account for this change.

Table 6-3 Observations:

\begin{tabular}{|l|l|l|l|}
\hline Cold (lower) temperature & 23 & {$\left[{ }^{\circ} \mathrm{C}\right]$} & \\
\hline Hot (higher) temperature & 69 & {$\left[{ }^{\circ} \mathrm{C}\right]$} & \\
\hline Tube eigenfrequency at 'cold' & $100 \%$ & {$[\mathrm{~Hz}]$} & Actual measurement \\
\hline Tube eigenfrequency at 'hot' & $101.21 \%$ & {$[\mathrm{~Hz}]$} & Actual measurement \\
\hline $\begin{array}{l}\text { Expected fractional change in sensitivity due } \\
\text { to hot-cold density change }\end{array}$ & 1.0028 & {$[-]$} & $\begin{array}{l}\text { Calculated as per } 6.2 .5 \\
\text { and 6.3.1 }\{\mathrm{A}\}\end{array}$ \\
\hline $\begin{array}{l}\text { Expected fractional change in sensitivity due } \\
\text { to temperature }\end{array}$ & 1.0113 & {$[-]$} & $\begin{array}{l}\text { Calculated as per 5.3.2 } \\
\{\mathrm{B}\}\end{array}$ \\
\hline Expected total fractional change in sensitivity & 1.0141 & {$[-]$} & $\{\mathrm{A} \cdot \mathrm{B}\}$ \\
\hline Measured relative sensitivity at 'hot' & 1.0127 & {$[-]$} & Actual measurement \\
\hline
\end{tabular}

Conclusion: In the test, it is seen that the sensitivity of the (example: type II) instrument changes by $\sim 1.3 \%$ for a temperature-change of $46^{\circ} \mathrm{C}$. This is the combined effect of density change and stiffness change. After correction, the residual error in sensitivity is around $0.2 \%$.

\subsection{Evaluation: ratiometric measurement}

\subsubsection{Inherent phase delays and mismatch in the instrument- construction}

It is proposed to measure the Coriolis force by means of the amplitudes' ratio of swing and twist motion of the Coriolis tube. The amplitudes' ratio, in turn, is determined from phasor angles. When the mass flow (and hence the Coriolis force) is zero, the mean phasor angle difference is expected to be $180^{\circ}$. At zero flow, the signal processing (that calculates the phasor angle) may register this as not being $180^{\circ}$ (i.e. $\pi$ rad) for two reasons:

- A force similar in nature (frequency and phase) to the Coriolis force acts upon the tube

- There exists an asymmetric phase-delay in the position sensing or signal conversion

For a type-II instrument, the phasor angle corresponding to a flow of $1 \mathrm{~g} / \mathrm{h}$ is about $150 \mu \mathrm{rad}$. It is desirable to have inherent phase-delays and mismatch significantly smaller than this for stable instrument performance. The mean phasor angles of several prototype instruments are measured. The measurements have:

\begin{tabular}{|l|l|l|}
\hline Mean of mean-phasor-angles & $(\pi-80 \mathrm{e}-6)$ & {$[\mathrm{rad}]$} \\
\hline Standard deviation & $40 \mathrm{e}-6$ & {$[\mathrm{rad}]$} \\
\hline
\end{tabular}

The mean (different than $\pi$ ) suggests a systematic offset in all instruments. This may be due to an actuator asymmetry or delay in the electronics, but is very small $(\sim 0.5 \mathrm{~g} / \mathrm{h})$ and hence merely corrected during instrument calibration. The standard deviation upon this offset too, is very small, indicating very little piece-to-piece variation.

It is noteworthy to state that in previous versions of the instrument (earlier iterations of the vmodel), where no transimpedance amplifier was employed for the optical interruption (tubeposition) sensors, the standard deviation in the zero-flow phasor angle measured for several instruments was orders of magnitude larger - about $600 \mathrm{e}-6$ rad. The parasitic capacitance of the photo-sensor was responsible for this, causing significant offset drift.

\subsubsection{Pole shift estimation algorithm}

By creating a shift in the rotation (excitation) axis, the pole-shift estimation (and correction) algorithm is tested. The shift is created by displacing the position-sensor ensemble WRT the oscillating tube. A reference instrument is used to feed the test instrument with a constant flow-rate. The MFR measured by the test instrument (expressed as a \%age of the reference flow) is logged against the measured sideways displacement of the sensor ensemble. 
Effect of rotation-axis shift (simulated by shifting sensor set)

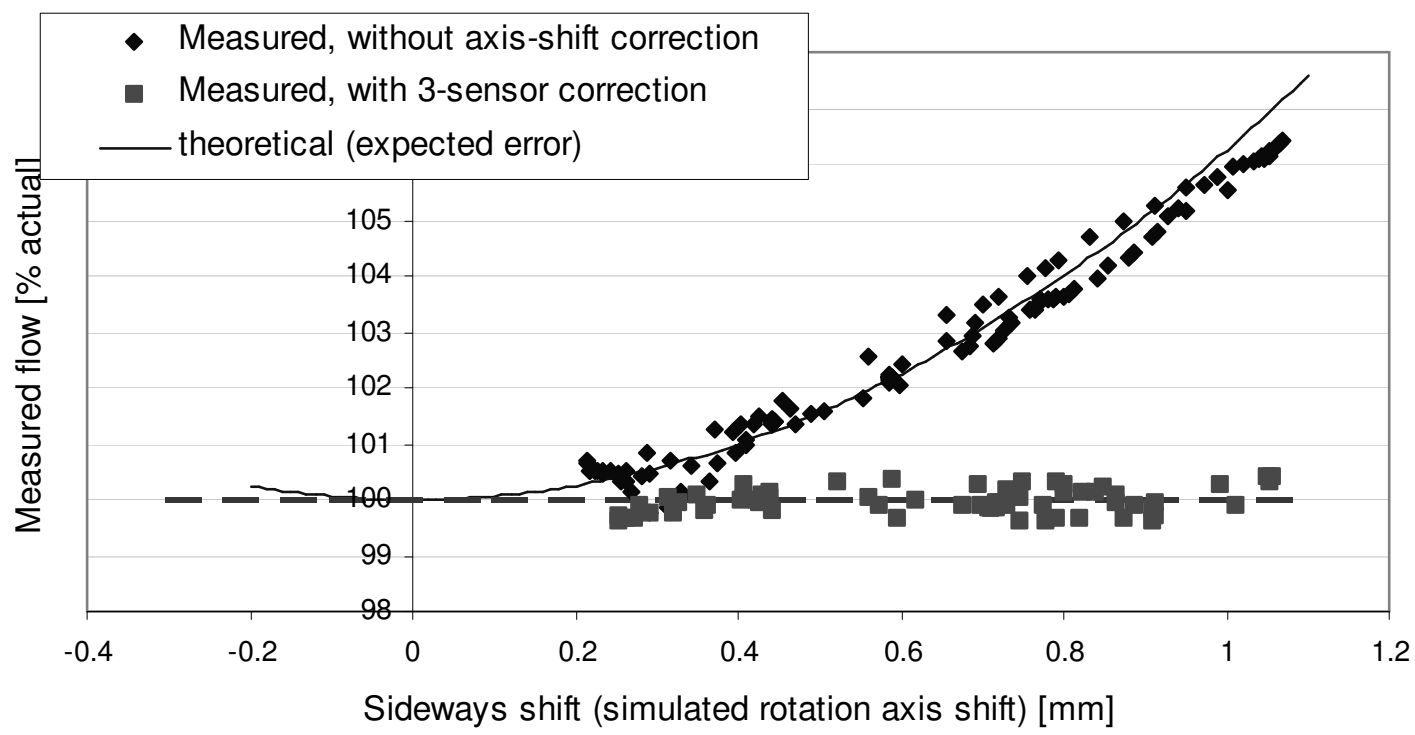

Figure 6.7 Rotation-axis shift: A comparison of expected measurement error, actual measurement (no correction) and measurement with '3-sensor compensation' correction

Without the correction, a large rate error is seen as a result of rotation-axis shift. A possible compensation method, based on phasor measurement with 3 position sensors has been demonstrated to reduce this error to less than $0.5 \%$ (for an axis shift of $1 \mathrm{~mm}$ )

\subsection{Measurements}

Qualification measurements carried out on the finished instruments are listed here. Correction factors for, e.g., the density, sensitivity, tube-temperature etc are incorporated in the measurement. The outputs of these instruments may be compared to the flow measured by a reference instrument as a final qualification. The results of these qualifications may be checked against the functional specifications (listed in Chapter 3.1).

\section{- The test equipment:}

Piston prover: This device measures volume flow rate (of gases) by means of an almost frictionless plunger moving in a cylinder. By measuring the gas temperature accurately and by application of the gas-law, the mass of the gas may be calculated indirectly.

Weigh-scales: measure the weight of liquid in a container placed upon it. Mass may be determined from weight; the mass flow rate may be estimated by differentiating readings over time. Mettler-Toledo analytical balance $-0.1 \mathrm{mg}$ resolution; lab balance $-1 \mathrm{mg}$ resolution

Thermal flowmeter instrument: a mass-flowmeter meter, calibrated for a certain fluid, used as a convenient reference.

Temperature controlled oven: for generating an environment from $-10^{\circ} \mathrm{C}$ to $+70^{\circ} \mathrm{C}$

Pressure vessel: Uses compressed air to drive a fluid into a target instrument under pressure. 
Control-pump: generates an output pressure controlled by a set-point; can be controlled by an instrument. Unlike the pressure vessel, the control pump can create pressurized fluid without any air (from the pressure source) dissolving into the fluid.

\subsubsection{Flow ranges}

The nominal flow ranges for instrument variants are listed below, In the following sections, the measurements relating to range will be based upon these values:

$\begin{array}{ll} & \text { Nomina } \\ \text { Type I } & 100 \mathrm{~g} / \mathrm{h} \\ \text { Type II } & 1 \mathrm{~kg} / \mathrm{h} \\ \text { Type III } & 10 \mathrm{~kg} / \mathrm{h}\end{array}$

\subsubsection{Uncertainty (in measuring flow)}

The relative measurement errors of several instruments are compared to a "conventional true value", measured by a reference-instrument. The outcome is listed in this section.

Measurements are carried out with several media - water, IPA, nitrogen, hydrogen, Argon, carbon dioxide, and helium.

The measured relative errors are plotted against the conventional true value; the "trumpet curves" shown correspond to a boundary (expressed as a combination of relative and absolute error) beyond which the error is larger than acceptable.

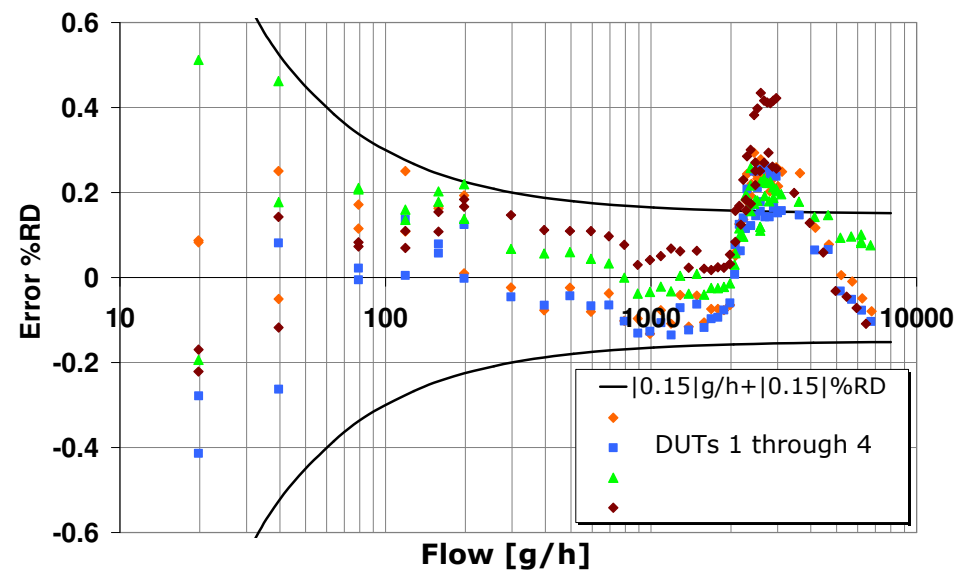

Figure 6.8 Water

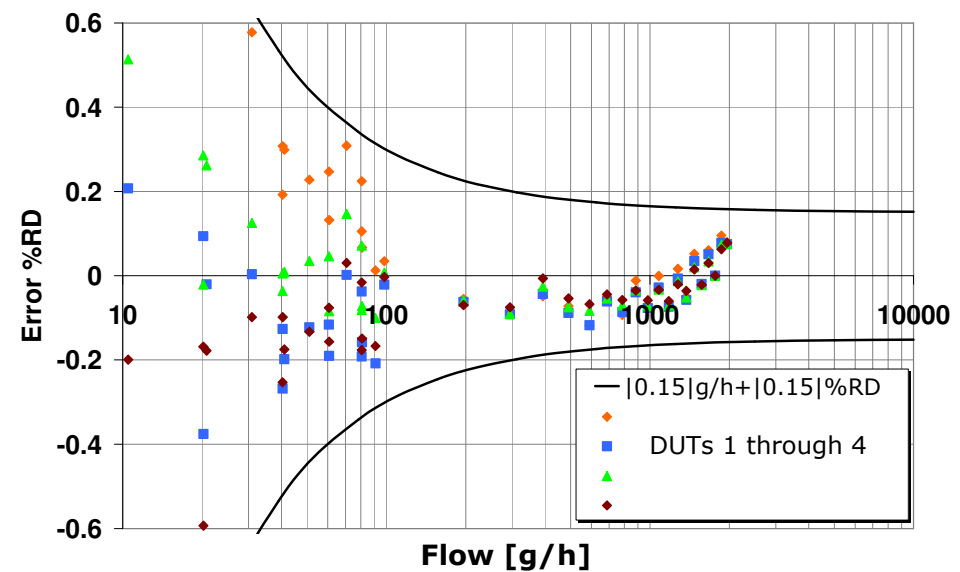

Figure 6.9 IPA 


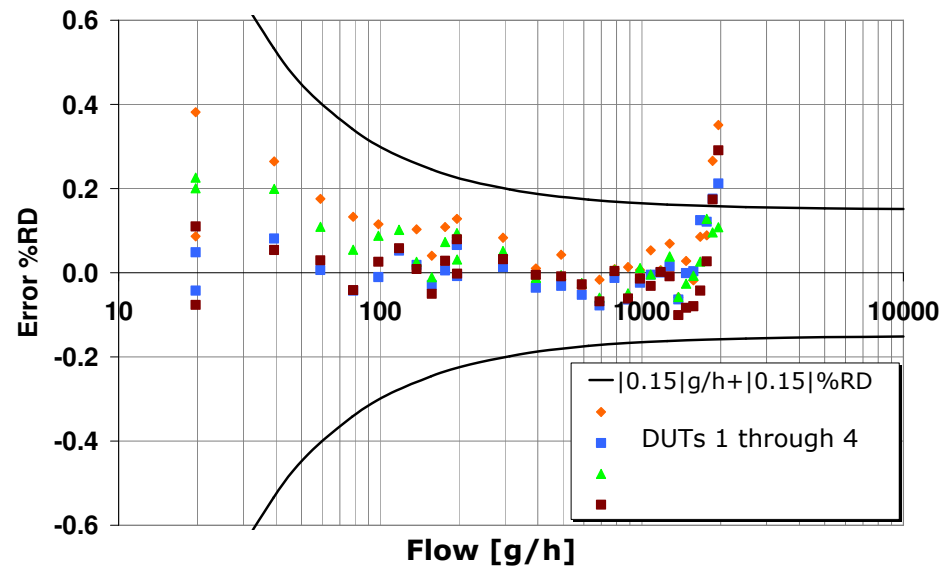

Figure 6.10 Methanol

Weigh-scales are used for the reference measurement. It is seen that up to the specified nominal flow-range $(1 \mathrm{~kg} / \mathrm{h})$, the measurement is linear and the relative error lies within the trumpet-curves. At around $3[\mathrm{~kg} / \mathrm{h}]$ for water and $2[\mathrm{~kg} / \mathrm{h}]$ for methanol, a reproducible artifact is seen on the error. This is probably a side-effect of transition to turbulent flow at these rates, and is a topic for further studies.

Similar tests are carried out for the five gases; here the reading of a piston-prover is used as the conventional true value.

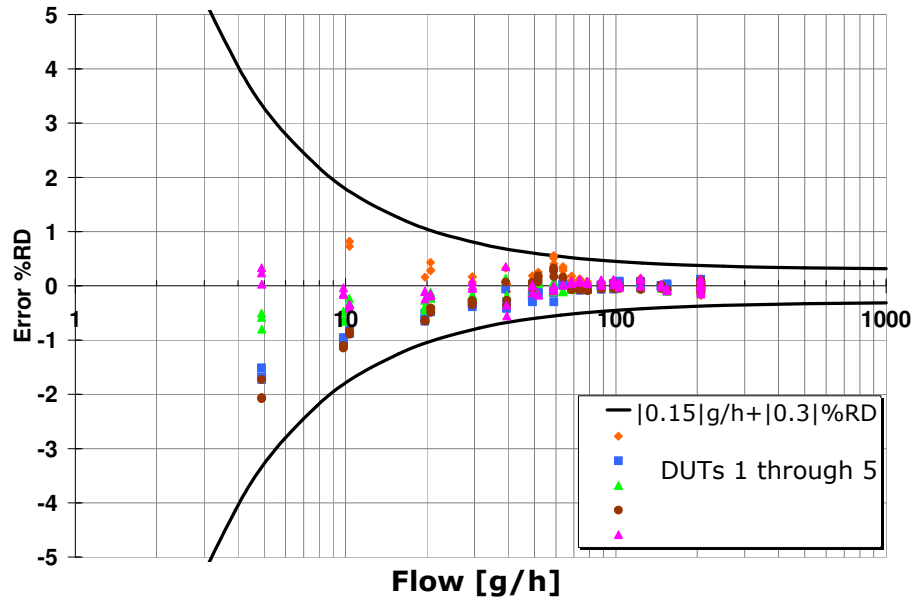

Figure 6.11 Nitrogen 


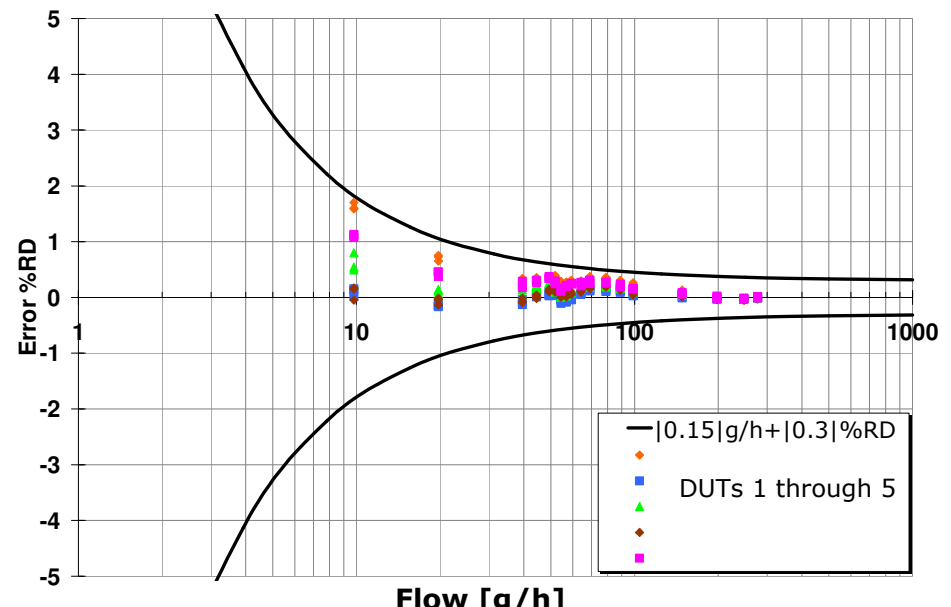

Figure 6.12 Carbon dioxide

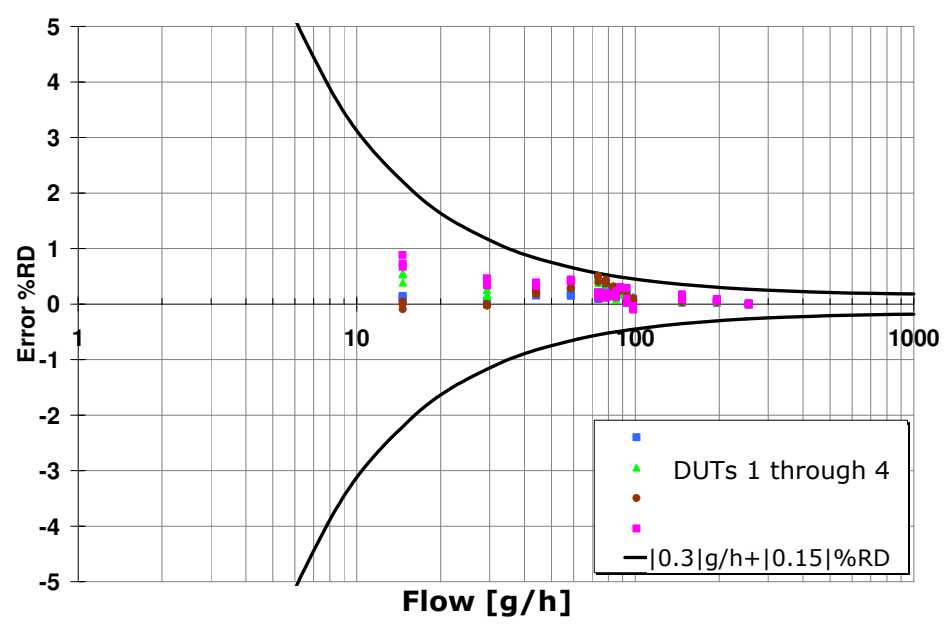

Figure 6.13 Argon

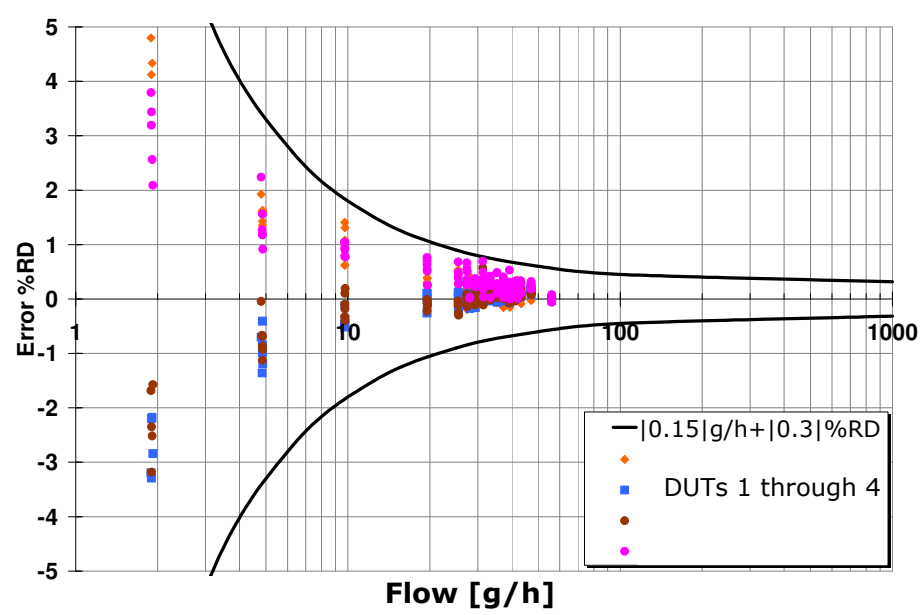

Figure 6.14 Hydrogen 


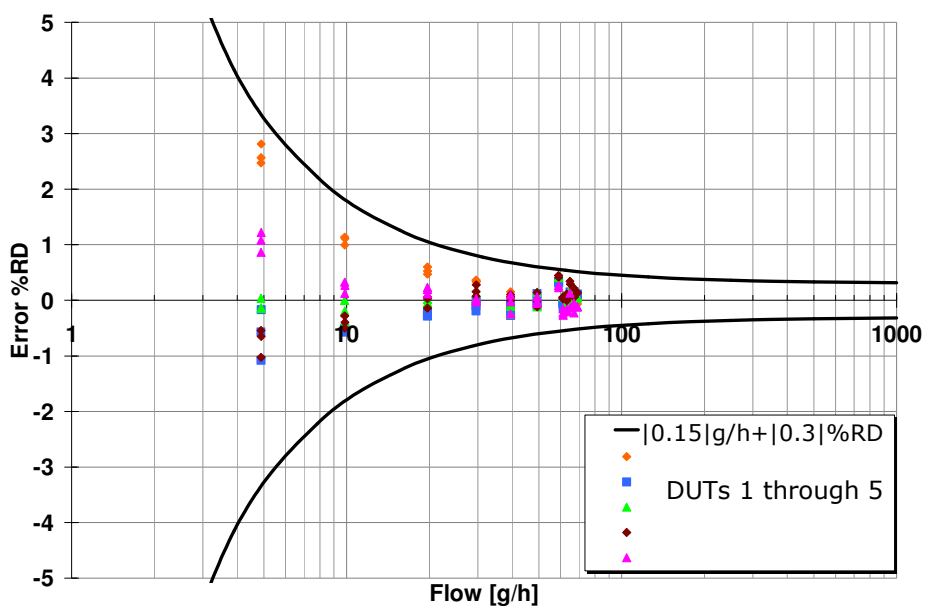

Figure 6.15 Helium

Conclusion:

For the tested type-II instruments, the measured error is:

\begin{tabular}{|c|c|c|}
\hline & Liquids & Gases \\
\hline $\begin{array}{l}\text { "Trumpet" envelope } \\
\text { (Actual measured) }\end{array}$ & $+/-(0.15 \mathrm{~g} / \mathrm{h}+0.15 \%$ reading $)$ & $+/-(0.15 \mathrm{~g} / \mathrm{h}+0.3 \%$ reading $)$ \\
\hline $\begin{array}{l}\text { Performance spec. } \\
\text { (Set out to achieve) } \\
\text { From Ch. } 3.1 .1\end{array}$ & $\begin{array}{l}\text { FS: } 2000 \mathrm{~g} / \mathrm{h} \\
\text { Zero stability: } 0.1 \% \mathrm{FS}, 2 \mathrm{~g} / \mathrm{h} \\
\text { Totalizer (rate) error: } \\
0.1 \% \text { of reading }\end{array}$ & $\begin{array}{l}\text { FS: } 100 \mathrm{~g} / \mathrm{h} \\
\text { Zero stability: } 0.1 \% \mathrm{FS}, 0.1 \mathrm{~g} / \mathrm{h} \\
\text { Totalizer (rate) error: } \\
0.1 \% \text { of reading }\end{array}$ \\
\hline
\end{tabular}

- Rate error: The "trumpet envelope" is slightly relaxed, compared to the specifications $(0.15 \%$ instead of $0.1 \%)$ to accommodate the turbulence artifact. For low flow rates, the $0.1 \%$ specification is in fact satisfied.

- Zero drift error: For gases, the requirement for zero-stability (expressed as a \%age of the full-scale) is relaxed from $0.1 \mathrm{~g} / \mathrm{h}$ to $0.15 \mathrm{~g} / \mathrm{h}$. The observed zero-stability $(0.15$ $\mathrm{g} / \mathrm{h})$ is much better than the specification for the higher flow-range $(0.1 \%$ of $2000 \mathrm{~g} / \mathrm{h}$ is $2 \mathrm{~g} / \mathrm{h}$ )

\subsubsection{Temperature effects: Drift and sensitivity}

\section{- Drift (zero-stability)}

In this experiment the drift (variation in measured flow at zero actual flow) of 10 instruments is monitored as a function of temperature. The temperature is increased from $0^{\circ} \mathrm{C}$ to $70^{\circ} \mathrm{C}$ and then decreased back to $0^{\circ} \mathrm{C}$ in $10^{\circ} \mathrm{C}$ steps. At each step, the temperature is held for 45 minutes. The temperature and the corresponding measured "zero flow" are logged at $20 \mathrm{~s}$ intervals. The temperature vs. time ( 3 examples) and the measured zero vs. time are shown in Figure 6.16 

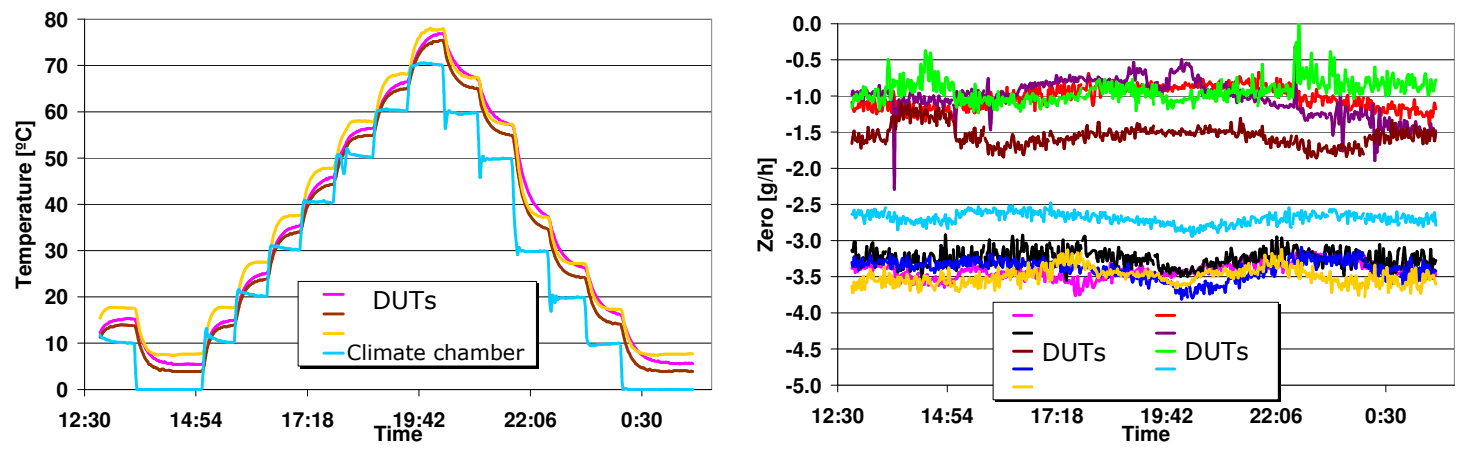

Figure 6.16 Temperature cycling - test results

Before this test, the instruments are intentionally not auto-zeroed, to have the traces separate $\&$ visible.

A plot of the drift as a function the temperature is shown in Figure 6.17; the mean has been subtracted from the reading, to highlight the variation related to instrument-temperature:

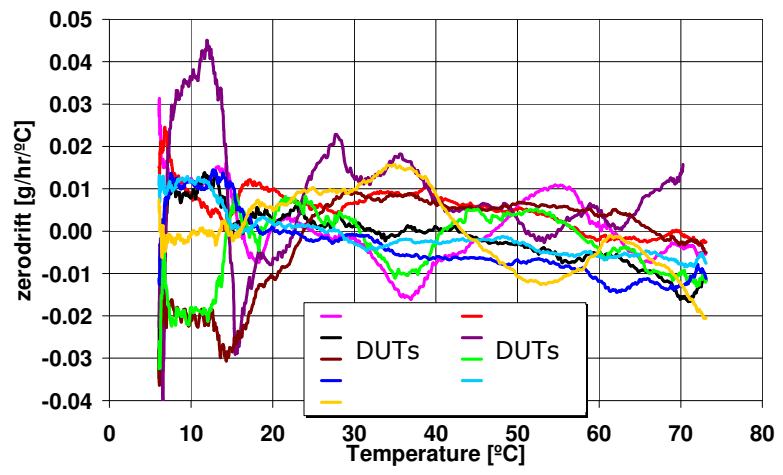

Figure 6.17 Offset drift as a function of environmental temperature

No significant trend is to be seen. The absolute drift is quite small $\left(<0.04 \mathrm{~g} / \mathrm{h}\right.$ PP above $\left.20^{\circ} \mathrm{C}\right)$, but somewhat larger at temperatures below $20^{\circ} \mathrm{C}$; the suspected cause is vibrations from the refrigeration-unit in the oven. It is noteworthy that the offset is hardly affected by the thermal shock (rapid $10^{\circ} \mathrm{C}$ change) at each temperature step.

\section{- Sensitivity (rate-stability)}

An algorithm to compensate the sensitivity for temperature variations has been proposed in 5.3.2 and subsequently evaluated in 6.3.2. The following experiment is devised to test the effectiveness of this correction algorithm:

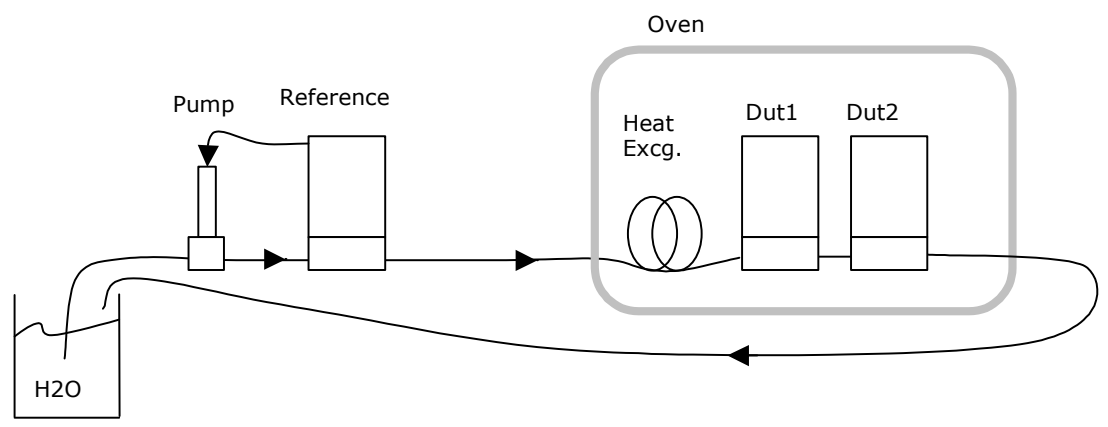

Figure 6.18 Sensitivity measurement - test set-up 
Two instruments are placed inside an oven; the oven is cycled between $0^{\circ} \mathrm{C}$ and $70^{\circ} \mathrm{C}$. A water-flow is generated by a pump and a reference instrument (treated as the conventional true value) and is forced through these two instruments. A heat-exchanger in the oven preheats the fluid. The readings of the two "DUT" instruments are compared with the reference. The following is seen:

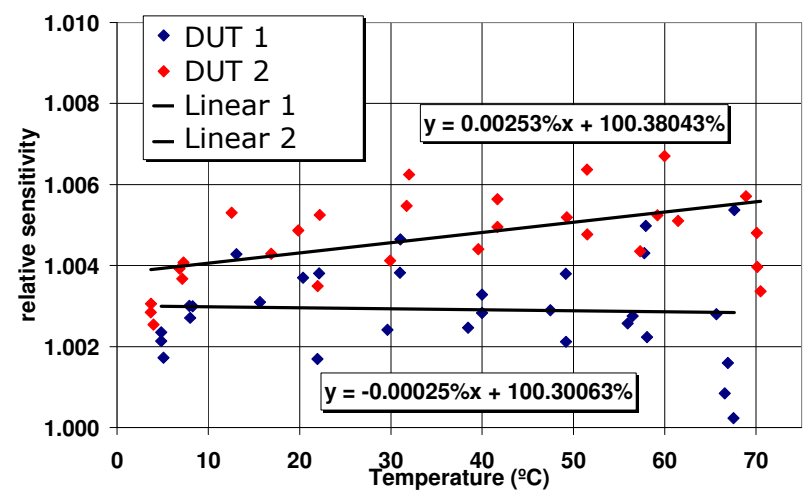

Figure 6.19 Instrument-sensitivity variation with temperature

We can conclude that the worse of the two instruments tested (the DUT 2) has a sensitivitydrift of $0.0025 \% /{ }^{\circ} \mathrm{C}$ - better than the target of $+/-0.01 \% /{ }^{\circ} \mathrm{C}$ from FR 10.0 (chapter 3.1.1)

\subsubsection{Measurement of medium density}

A two-point calibration based upon the discussion in 6.2.5 with dry nitrogen and with water is carried out for all instruments. Subsequently, the calibrated instruments can be used to measure the density of unknown fluids.

In tests carried out with four gases (other than nitrogen) and two liquids (other than water), the following is seen (the conventional true values are taken from gas and fluid charts).
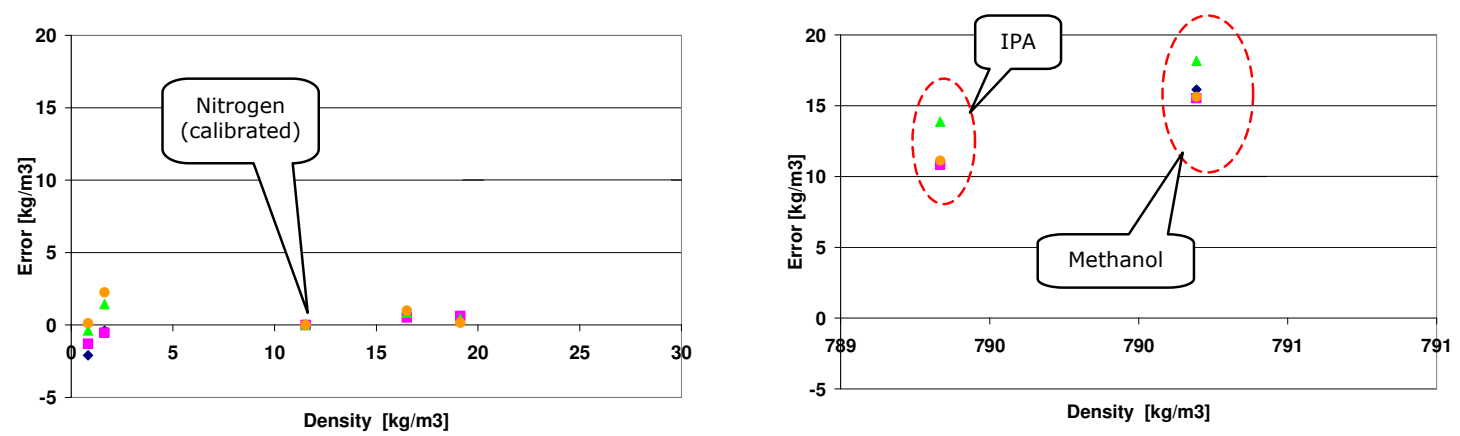

Figure 6.20 Density measurement errors for various media

It is seen that the calculated values agree with the reference values for the respective gases to within $+/-2 \mathrm{~kg} / \mathrm{m}^{3}$ and fluids to within $20 \mathrm{~kg} \cdot \mathrm{m}^{3}$. The extra density measured with IPA and methanol is suspected to be caused by dissolved gases (compressed air used to pump the medium).

A second test is carried out to test density measurement when density variation is caused by medium heating. Water is pumped into two test instruments placed in an oven. The water is preheated by means of a heat exchanger placed inside the oven. The oven temperature is cycled between $5^{\circ} \mathrm{C}$ and $70^{\circ} \mathrm{C}$ 

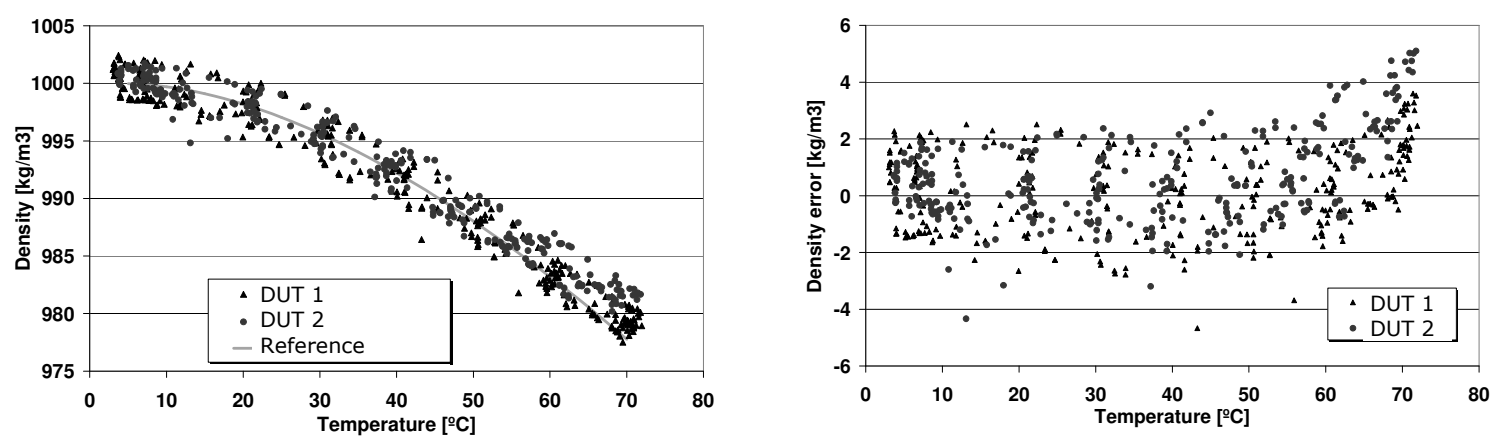

Figure 6.21 Density measurement with varying temperature

Density measurement, based upon the three-parameter estimation proposed in 5.2 .5 is seen to perform well (error $<5 \mathrm{~kg} / \mathrm{m}^{3}$ i.e. $<0.5 \%$ ) even in presence of large temperature change $\left(65^{\circ} \mathrm{C}\right)$. This is better than the FR17.0 requirement of $10 \mathrm{~kg} / \mathrm{m}^{3}$.

\subsubsection{Medium density: effect on sensitivity}

The density compensation method proposed in 5.3.2., the principle of which is evaluated in 6.3.1 is implemented in test instruments. The sensitivity of the instrument is measured, against a reference instrument for various media. It is seen that the sensitivity error for liquid media is within $0.2 \%$ of the reading and for gases, within $0.5 \%$ of the reading.

\subsubsection{Long-duration test of drift and sensitivity}

The offset (zero drift) and the sensitivities of three type II instruments are tested over a period of three months.

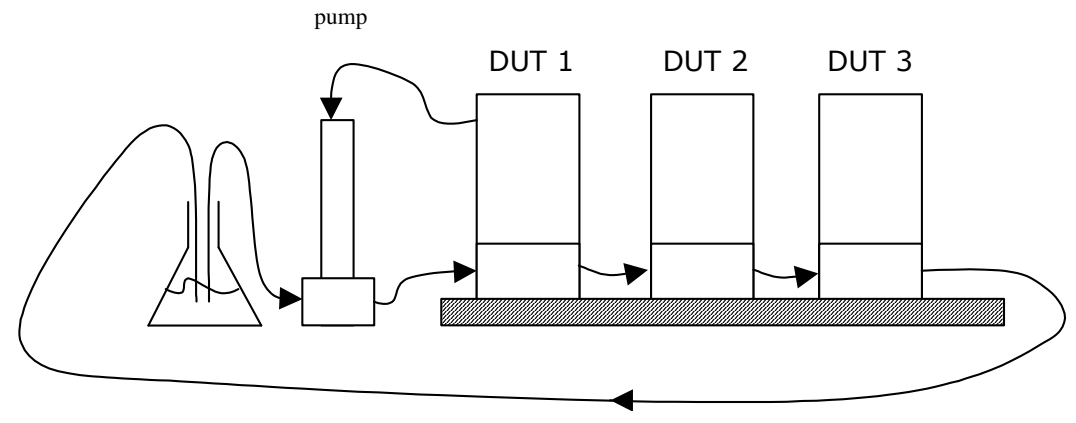

Figure 6.22 Test set-up

One of the three instruments is used to create a pattern of flow to avoid stagnation, and to simultaneously measure the offset (the reading at zero actual flow) and the sensitivity (reading of two instruments WRT the reference third instrument). 

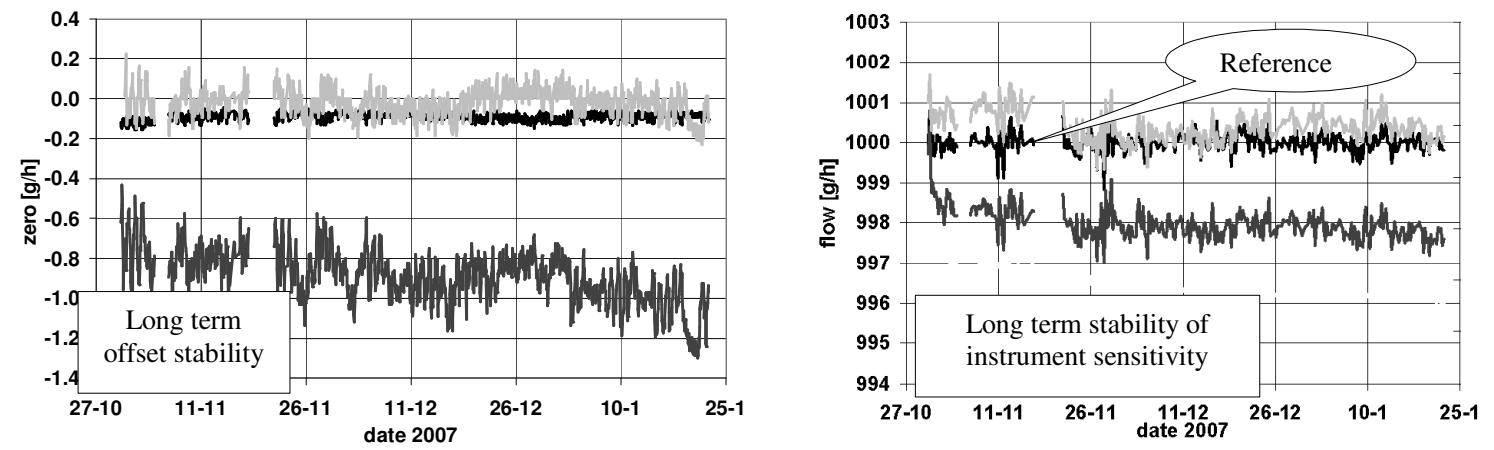

Figure 6.23 Long term stability plots

During the measurement period, the ambient temperature varies about $10^{\circ} \mathrm{C}$. The following observations can be made:

- For all the instruments, the offset-drift over 3 months is less than $0.2 \mathrm{~g} / \mathrm{h}$

- $\quad$ For one of the three instruments, the offset calibration was off, at $-0.9 \mathrm{~g} / \mathrm{h}$

- Correcting for the faulty offset, the sensitivity of the three instruments is within $0.2 \%$ of one another

- The relative change in sensitivity of each instrument over 3 months is less than $0.1 \%$

\subsubsection{Noise on the measured flow}

From in-system tests carried out on a non-actuated type II tube (see 5.4.3) the equivalent inband random Coriolis-mode-like motion of the tube due to the combined effect of environmental vibrations, EM pickup and component noise (sensor, amplifier and quantizer) is about $5 \mathrm{~nm}$ RMS. For typical instrument amplitude settings, this corresponds to a flowmeasurement noise of approximately $1 \mathrm{~g} / \mathrm{h}$. A set of ten instruments is tested for noise. The instruments are operated at several (constant) flows, the readings are logged, and the standard deviation upon the mean value at each flow is determined

- The worst amongst the ten instruments has RMS noise of $0.67 \mathrm{~g} / \mathrm{h}$. The average RMS noise is about $0.56 \mathrm{~g} / \mathrm{h}$.

- The presence of a (constant) flow has negligible influence on the measurement noise

- The ambient temperature has little effect on the measurement noise. This is not surprising, as the noise from thermo-electrical sources is a small fraction of the total noise.

\subsubsection{Meter response time}

The response of the instrument to a step change is monitored by means of a toggle-valve. As the resistance of the valve in its on state is quite small, no significant hydraulic time-constants are anticipated. 


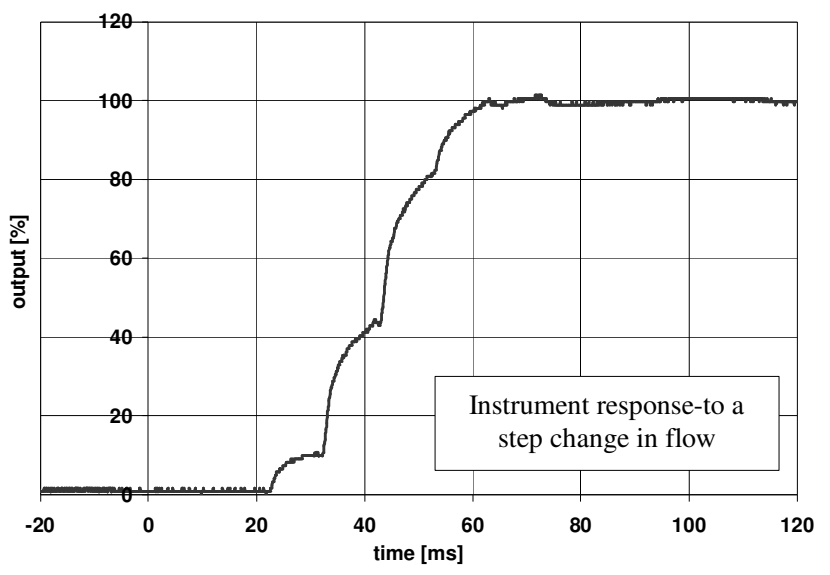

Figure 6.24

Here, the quadrature-detector filter is set at $25 \mathrm{~Hz}$. The valve is switched on at $t=0$. The output is presented to an oscilloscope as an analog voltage signal by means of a D/A converter. The dead-time of the D/A converter is about $20 \mathrm{~ms}$. This is visible as the initial delay. The update rate of the D/A converter (visible as steps) is about $10 \mathrm{~ms}$. The antialiasing filter time-constant (of the D/A converter) is about $5 \mathrm{~ms}$. Measured with the D/A converter (standard hardware), the instrument is seen to settle to within $2 \%$ of the final value well within $0.1 \mathrm{~s} \mathrm{(Ch.} \mathrm{3.1.1} \mathrm{FR} \mathrm{8.0).} \mathrm{Neglecting} \mathrm{the} \mathrm{delay} \mathrm{of} \mathrm{the} \mathrm{output} \mathrm{D/A} \mathrm{converter,} \mathrm{the} \mathrm{core}$ signal processing seems to respond to a step change within $40 \mathrm{~ms}$; this agrees well with the $25 \mathrm{~Hz}$ filter setting. Naturally, it is possible to trade off response speed for noise.

\subsubsection{Pressure drop and effects of absolute pressure on sensitivity}

The pressure drops across the type I, type II and type III instruments (M12, M13 and M14 respectively in Figure 6.25) are measured for various flows, for air and water:

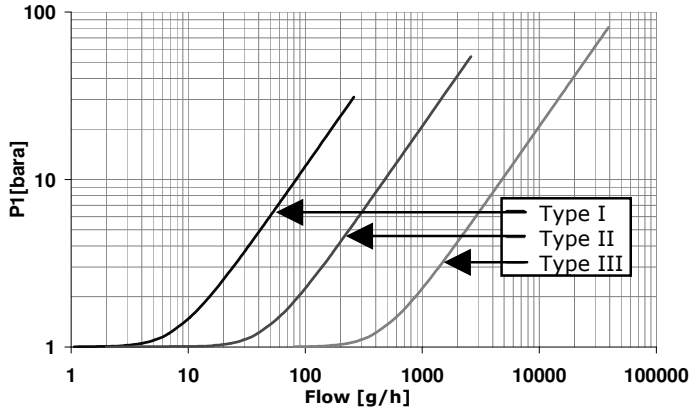

(Air: absolute pressure at inlet)

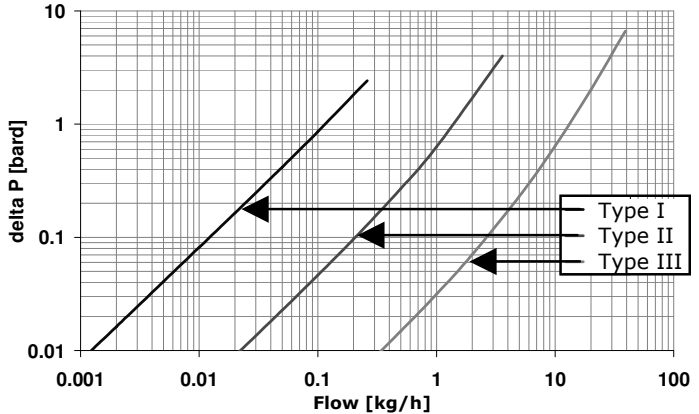

(Water: differential pressure across instrument)

Figure 6.25 Pressure drops at different flow-rates for air and for water

It is seen that for a pressure drop of 0.5 and 1 bar across the instrument, the three instrument types give:

Table 6-4

\begin{tabular}{|l|l|l|l|l|}
\hline & Air: 0.5 bard & Air: 1 bard & Water: 0.5 bard & Water: 1 bard \\
\hline Type I & $10 \mathrm{~g} / \mathrm{h}$ & $15 \mathrm{~g} / \mathrm{h}$ & $60 \mathrm{~g} / \mathrm{h}$ & $100 \mathrm{~g} / \mathrm{h}$ \\
\hline Type II & $60 \mathrm{~g} / \mathrm{h}$ & $90 \mathrm{~g} / \mathrm{h}$ & $800 \mathrm{~g} / \mathrm{h}$ & $1.3 \mathrm{~kg} / \mathrm{h}$ \\
\hline Type III & $600 \mathrm{~g} / \mathrm{h}$ & $900 \mathrm{~g} / \mathrm{h}$ & $9 \mathrm{~kg} / \mathrm{h}$ & $13 \mathrm{~kg} / \mathrm{h}$ \\
\hline
\end{tabular}

Though some of these values are somewhat smaller than the specification, they are acceptable. 
The error of the instrument is measured as a function of the inlet pressure, while the DUT itself controls the air-flow:

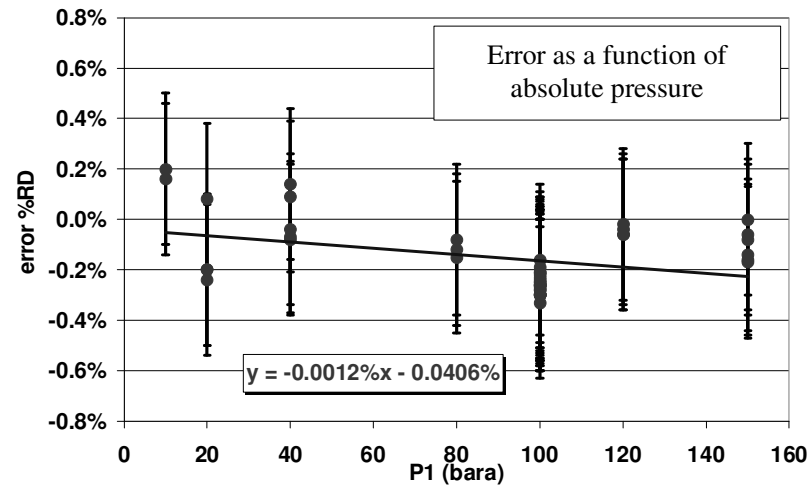

Figure 6.26 Error as a function of the absolute pressure

Little influence of the inlet pressure is seen. A Piston prover instrument is used as the reference here; the reliability of the reference is doubtful, as the high inlet pressures necessitate large decompression, meaning substantial cooling; the uncertainty of the piston prover itself is largely influenced by temperature.

\subsubsection{Influence of instrument attitude on offset and sensitivity}

An experiment is carried out to test the influence of instrument-attitude on the sensitivity. Two instruments are setup in series where one instrument is put in various positions to determine its sensitivity as a function of the attitude. This test shows the result for both zero stability and deviation of sensitivity for various attitudes.

Table 6-5 - Change in sensitivity with instrument attitude

\begin{tabular}{l|cccc} 
attitude: & experiment 1 & experiment 2 & repeatability & average \\
\hline straight up & $0.00 \%$ & $0.01 \%$ & $0.01 \%$ & $0.01 \%$ \\
left side & $-0.01 \%$ & $0.01 \%$ & $0.01 \%$ & $0.00 \%$ \\
right side & $0.06 \%$ & $0.07 \%$ & $0.01 \%$ & $0.07 \%$ \\
belly & $0.03 \%$ & $0.04 \%$ & $0.01 \%$ & $0.03 \%$ \\
back & $-0.03 \%$ & $-0.02 \%$ & $0.01 \%$ & $-0.03 \%$ \\
upside down & $0.07 \%$ & $0.06 \%$ & $-0.01 \%$ & $0.06 \%$
\end{tabular}

The outcome of the test shows that for the sensitivity:

- The experiment is very repeatable within $0.01 \%$

- The maximum change in sensitivity is $<0.07 \%$.

- The typical change in sensitivity is $<0.04 \%$

The shift in sensitivity results in an error in reading. A comparison between two opposite positions show that the worst-case change in sensitivity change stays within $0.07 \%$ as depicted in the table below: 
Table 6-6 Opposite-attitude pairs: change in sensitivity

\begin{tabular}{l|c} 
& Difference \\
\hline straight up $<>$ upside down & $-0.06 \%$ \\
left side $<>$ right side & $-0.07 \%$ \\
belly $<>$ back & $0.06 \%$
\end{tabular}

Table 6-7 Offset-shift with attitude (all values $[\mathrm{g} / \mathrm{h}]$ )

\begin{tabular}{l|cccc} 
& experiment 1 & experiment 2 & repeatability & average \\
\hline straight up & 0.11 & 0.14 & -0.03 & 0.12 \\
left side & 0.33 & 0.51 & -0.18 & 0.42 \\
right side & 0.07 & 0.27 & -0.20 & 0.17 \\
belly & -0.27 & -0.70 & 0.43 & -0.48 \\
back & 0.09 & 0.36 & -0.27 & 0.22 \\
upside down & 0.07 & 0.02 & 0.05 & 0.04
\end{tabular}

The position dependent change in offset is less than $1 \mathrm{~g} / \mathrm{h}$. Since the instrument needs to be zeroed at process conditions (including its position) the outcome of this experiment, though interesting, is of little consequence.

\subsection{Conclusion}

In chapter 6 , various concepts are tested and evaluated. In section 6.5 , the performance of a set of instruments is investigated in detail as a qualification of the specifications stated in chapter 3 . This rounds up the last iterative stage in the V-model of system engineering. 


\section{Conclusions and recommendations}

\subsection{The output of the project}

This thesis is aimed at the design of a Coriolis mass-flow meter intended for low flow-rates. A broad range of topics is treated from the Mechatronics perspective - ranging from tube-shapes and eigenmodes to sensor and actuator design to data processing and presentation. Based upon a set of concept and design choices - evaluated \& selected from a number of existing and novel ideas - three scale variants (having the same basis design components) are worked out in detail. One of these three, the type II instrument, is illustratively discussed in detail in this thesis. Based upon the concepts and design elements resulting from the present research, a commercial instrument has been launched. Several patents have been assigned, and several others are pending.

\subsection{Conclusions at the system-level}

- An instrument capable of measuring low mass-flow-rates $(100 \mathrm{~g} / \mathrm{h}$ full-scale) has been demonstrated. This is the cumulative result of choices made at the subsystem and component levels and suitable system integration thereof.

- A zero (offset)-drift better than $0.1 \mathrm{~g} / \mathrm{h}$ and a relative error against conventional reference instruments better than $0.2 \%$ has been demonstrated.

- A measurement scheme for Coriolis force based on ratiometric measurement purely in the time-domain has been demonstrated - at no point is any amplitude information used as a measure for the mass flow. This eliminates the need for component-level calibration. As the Coriolis force is miniscule compared to the actuation and oscillation forces, the high precision (1:1e5) afforded by cheap time-references helps isolate the tiny Coriolis forces (magnitudes).

- The construction of the demonstrated instrument is possible using conventional finemechanical techniques (as opposed to micro-machining etc.) and COTS sensors and processing electronics, with low production-costs to target the mass-market.

\subsection{Conclusions at the subsystem level}

- In a Coriolis meter using an unconstrained oscillating tube, the ratio of the amplitude of the Coriolis response motion -the "swing"- to the amplitude of excitation motion -the rotation or "twist"- is an indication of the Coriolis force. It is an insufficient approximation to relate the Coriolis force to the phasor-angle or time shift between position signals.

- The sensitivity of the Coriolis mass flow meter discussed in this thesis varies as a function of the medium-density. This variation is the result of choices such as excitation at eigenfrequency and Coriolis response on the mass line. Despite the variation, these choices are made as they afford several advantages. The variation is well explainable and a correction based on physical principles (rather than trial-and-error methods) is demonstrated.

- The sensitivity of the Coriolis mass flow meter also varies as a function of the temperature (primarily that of the tube material). Correction for this variation, based upon the underlying physics, has been demonstrated.

- In order to increase instrument sensitivity to Coriolis motion, it is advantageous to have as small a common-mode signal as possible. For this reason, it is advantageous to place the tube-motion sensors close to the rotation axis. A shift in the rotation axis affects the ratiosensitivity. To correct for such a shift, using only time-domain (phasor) measurements, three (or more) position sensors are essential.

\subsection{Conclusions at the component-level}

- By using a tube with a high Q-factor (>1000) excited at its eigenfrequency, the excitation effort is minimized, also minimizing the effect of actuator asymmetry on the Coriolis-mode motion. 
- A meter tube-shape whose geometry changes little due to temperature is realized by means of a bent tube where the inlet and outlet are collocated.

- A shape for the resonant tube, whereby the 'spring' function is unified (single torsion spring entity) and isolated from the 'rotating tube' function affords predictable and constant performance (sensitivity and offset-stability).

- It is advantageous to choose a shape for the resonant tube where the excitation eigenfrequency and Coriolis (response) eigenfrequency are in a ratio $\mathrm{R}$ such that $1.5<R<1.8$. The lower limit (1.5) ensures that the response motion (gain) is not significantly affected by a change in the Q-factor of the oscillating tube. The upper limit (1.8) ensures that the response motion is not too far away (in our case, on the mass line); this would mean a smaller gain.

- Tuning the ratio of the excitation and response modes is easily possible by tuning the aspect-ratio of the rectangular tube-shape.

- A novel contactless actuation principle based on induction and Lorentz forces is demonstrated for actuation of the tube. The contactless nature makes near-ideal actuation (without attachment asymmetries) possible.

- A torque actuator construction is demonstrated, which due to the translation property of torque, makes for a near-ideal actuator.

- The position of the actuator, very close to the rotation-axis of the response motion, introduces an additional attenuation preventing the actuation of the Coriolis motion.

- Contactless sensing is carried out by means of optical interruption sensors. Time delays inherent to these devices, caused by parasitic capacitance, are eliminated by the use of transimpedance amplifier techniques.

- The sensor signals are interpreted by quadrature lock-in phase detection technique - this technique inherently ignores harmonics, and hence non-linearity of the sensor signals is inconsequential.

- $\quad$ Due to the quadrature lock-in phase detection technique, measurement happens in a narrow frequency band around the excitation frequency. Being away from DC, this inherently gets rid of flicker ( $1 / \mathrm{f})$, and being a narrow-band measurement, it also affords low measurement noise.

- A simple technique for creating sustained stable oscillations in the Coriolis tube is demonstrated.

\subsection{Recommendations}

Research into the following issues can further improve the instrument performance:

- Investigating laminar>turbulent transition It is seen for some media that the transition from laminar flow to turbulent flow occurs within the typical flow range. It is observed (see Ch. 6.5) that during this transition the instrument shows a slightly higher sensitivity and the Q-factor of the tube too becomes substantially lower. A possible explanation is that the flow resistance is modulated by the tube oscillation leading to pulsating (at tube eigenfrequency) flow. This phenomenon merits investigation.

- Optimization algorithm for the tube shape

Due to the multitude of parameters that may be varied, finding the ideal tube shape is a challenge. By means of an automated "goal seeking" algorithm, it may be possible to come up with a better tube shape than those presented in this work. Naturally a new challenge will be to define all possible criteria, cost functions, and boundaries for such an algorithm.

- Internal and external vibrations

All Coriolis flowmeters inherently have a moving flow-tube. Vibrations generated due to the motion of this tube and external vibrations can potentially interfere with the measurement of the generated Coriolis force. An investigation into options to reduce the effect of external vibrations would add significantly to the present work.

- $\quad$ Reducing instrument noise

With the present device (type-II), at maximum bandwidth, the lower limit on measuring flow is set by the noise at about $1 \mathrm{~g} / \mathrm{h}$. For applications with short interval times, it is necessary to reduce the noise by improving signal and data path, especially the instrument-ground-and-shield and converter clock jitter. 
- Detailed magnetic modeling

A Lorentz actuation is chosen for of the Coriolis tube in the present work. Detailed analysis of the magnetic quantities and interaction with the other instrument components (leakage fluxes etc.) would be useful for application of this concept to a range of instruments. 



\section{Appendix A - Ratiometric measurement with phasors}

\section{The principle of ratiometric measurement based on phasors can be summarized as:}

Actuators placed around a Coriolis tube excite it in a rotation (twist) oscillation mode. The tip of the tube describes an angular motion:

$$
\theta_{\text {twist }}=\theta_{\max } \sin \left(\omega_{\text {eig }} \cdot t\right)
$$

The z-motion of a point $n$ on the tube at a distance $r_{n}$ from the center of rotation due to this rotation excitation (approximated to a linear scale for small values of $\theta$ ) may be written as

$$
z_{n(t w)}=r_{n} \cdot \theta_{\max } \sin \left(\omega_{\text {eig }} \cdot t\right)=p_{n} \sin \left(\omega_{\text {eig }} \cdot t\right) \text { [say] }
$$

Now, the Coriolis force causes a swing motion, $90^{\circ}$ out-of-phase with the motion described in Eq. A.2. If a given mass flow causes a Coriolis motion of amplitude, say $q$, this tube motion (swing or translation, thus equal at all radial distances) may be written as:

$$
z_{\text {cor }}=q \cos \left(\omega_{\text {eig }} \cdot t\right)
$$

The total resultant motion of the tube as seen by a position sensor measuring the z-position of the tube at $n$ is a superposition of Eq. A.2 and Eq. A.3. Thus:

$$
z_{n}=z_{n(t w)}+z_{\text {cor }}=p_{n} \sin \left(\omega_{\text {eig }} \cdot t\right)+q \cos \left(\omega_{\text {eig }} \cdot t\right)
$$

Consider that the position is sensed by two elements on either side of rotation axis as seen in fig. A.1. Equation A.4 may be represented as a phasor:
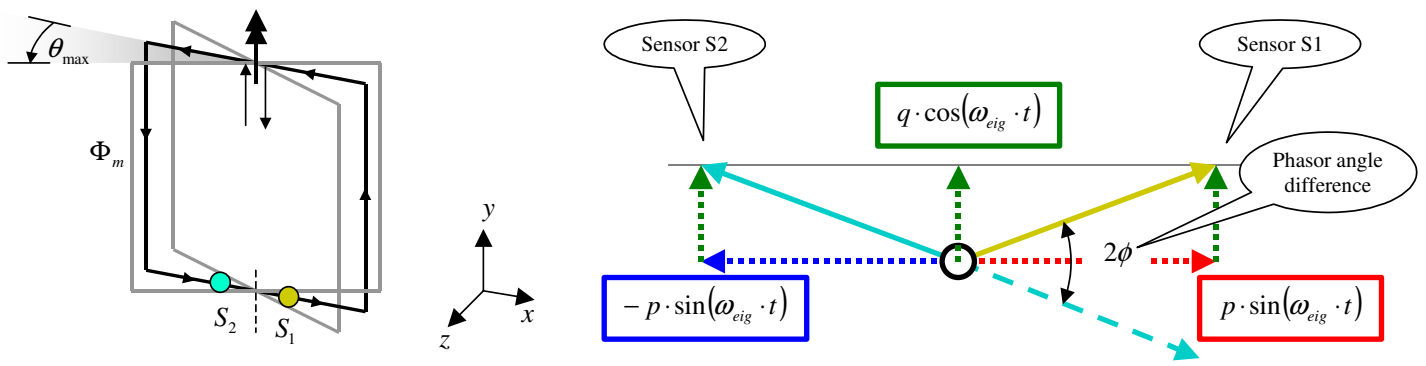

Figure A.1 Phasor representation of deflection (seen by sensors)

Having a time domain representation:

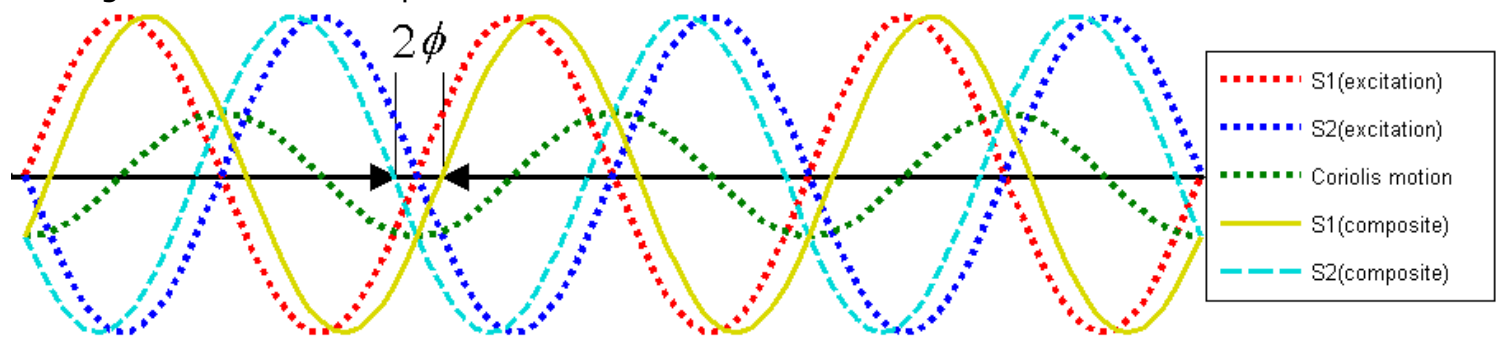

Figure A.2

Here, the measured phase difference between two sensor signals S1 and S2 is the composite $(\pi-2 \phi)$, where $\phi=\tan ^{-1}\left(\frac{q}{p}\right) \approx \frac{q}{p}$ - the amplitudes' ratio. 



\section{Appendix B - Lorentz actuation}

\section{B.1 Dimensioning of the stator yoke of the Lorentz actuator}

A static field yoke provides a pair of air-gaps with anti-parallel fields. A current-carrying conductor passing through these two gaps will experience a force couple (a pair of equal and opposite Lorentz forces). This principle is used to actuate the Coriolis tube in the type-II apparatus in a torsion-mode excitation.

To make a compact construction, rare earth (NdFeB) magnets are used for the yoke (high residual flux density). The two air-gaps in the yoke must be identical in gap-width and field strength (antiparallel) in order to have a pure torque. Hence, the magnetic circuit of the yoke is constructed in such a way that the two air-gaps are 'connected' in series, instead of using two separate constructions. Being in series, the flux passing through each gap is equal.

Further, soft iron is used as the material for the yoke, as it has a saturation flux rating that is suitable for use with NdFeB magnets, and is easily available and machineable.

\section{Geometry}

NdFeB magnets are typically available in 1.1 to 1.2 Tesla (residual flux density) rating. Flux density ( $\mathrm{B}[\mathrm{Tesla}]$ ) is a metric of the strength of a magnetic field. Two other metrics describe the magnet: the surface area and the thickness. The surface area decides the total flux available from the magnet (flux density $\times$ area $=$ total flux). The flux "coming out" of the magnet may be spread or concentrated using appropriate construction of a 'pole shoe' of a ferromagnetic material (in this case - soft iron).

The residual flux density mentioned above (1.2 Tesla) is available from a magnet only if a high permeability (analogous to low resistance) path is made available between the two poles of the magnet. The total flux ( $\Phi$ [Weber]) is equal at all cross sections of such a magnetic path (irrespective of area). Now, for example, if the cross section of the high permeability 'short circuit' path is constant over the entire length, the flux density (Tesla) is equal in the whole path. In practice, if there is an air-gap in the magnetic path, it has the effect of reducing the flux and hence also the flux density along the entire path in a ratio expressed by

$$
B_{\text {path }}=B_{\text {gap }}=B_{\text {res }} * \frac{l_{\text {magnet }}}{l_{\text {magnet }}+l_{\text {gap }}}
$$

Where $B_{\text {res }}$ is the residual flux density (1.2 Tesla, in this case), and $I_{\text {magnet }}$ is the thickness of the magnet along the direction of polarization.

Thus, in case of a magnetic circuit with an air-gap, a thicker magnet causes higher flux and hence higher flux density along the entire path (which can be best seen in the air-gap). Further, 'spreading' the air gap to double the area has the same effect as halving the gaplength (analogous to resistance). Thus a gap with double area causes more flux, (though lower flux density within the gap). Consider the following example:

$$
B=1.2 * \frac{l}{l+l}=0.6[\text { Tesla }]
$$$$
B=1.2 * \frac{l}{l+l / 2}=0.8[\text { Tesla }]
$$
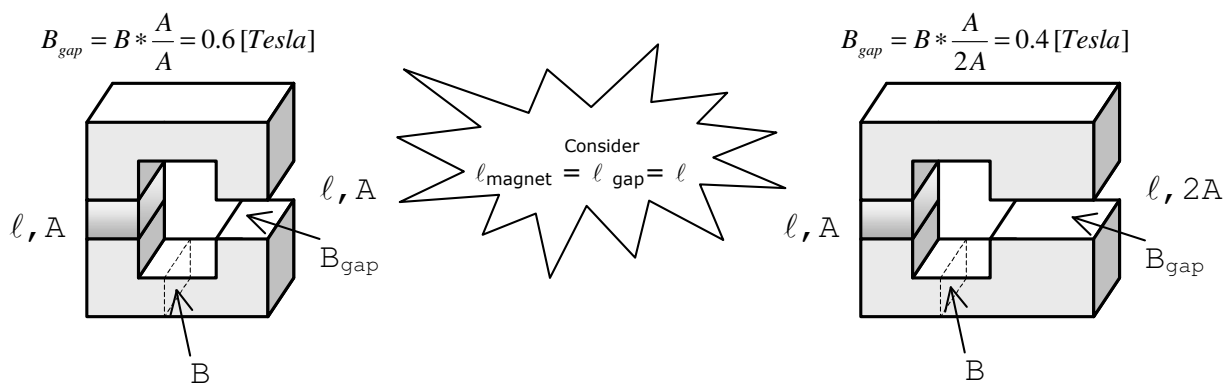

Figure B.1 Illustration: Flux density in magnetic yoke with air-gap 
Also, if several equal-area air gaps are present in series, their effective 'resistance' (length) adds, but there is no 'spreading' effect (obviously, since flux in each gap is equal). Further, since the air-gap areas are the same, the flux densities will also be equal.

\section{Magnetic flux ( $\Phi)$ :}

Generally, if an NdFeB magnet of pole surface area $A_{\text {magnet }}$ and thickness $I_{\text {magnet }}$ is placed in a magnetic path as shown in Figure B.1, then the total flux in the path is approximately ${ }^{27}$ given by the expression:

$$
\Phi_{\text {path }}=\left(\frac{\left.1.2 * A_{\text {magnet }}\right)}{[\text { Tesla }]} \frac{l_{\text {magnet }}}{l_{\text {magnet }}+l_{\text {gap }}\left(A_{\text {magnet }} / A_{\text {gap }}\right)}\right.
$$

Where $I_{\text {gap }}$ is the equivalent total gap length and $A_{\text {gap }}$ is the air-gap pole surface area

\section{Creating a flux (density) in an air-gap:}

To create a magnetic air-gap, we need to first create a 'path' using a ferromagnetic material around a magnet. If a magnetic path has a 'break' (e.g. - as shown in figure 2), where the equivalent gap-length is much smaller than the thickness of the magnet itself, most of the flux coming 'out' of the magnet will choose to travel through the gap. Some flux will leak through the surrounding regions, either directly from the magnet or from parts of the magnetic path. The main idea is to design a path such that most of the flux passes through the 'break' in the magnetic path, i.e. the air-gap. Adding magnetic 'insulators' (diamagnetic materials) to block leakage fields is usually impractical.

\section{Flux density (B):}

In the example above, if the path cross-section area at some point (including in the air-gap) is $\mathbf{A}_{\mathbf{c s}}$, then the flux density (B) perpendicular to that cross-section is:

$$
B_{C S}=\Phi_{\text {path }} / A_{C S}
$$

For NdFeB magnets, as stated earlier, the residual flux density is about 1.2 Tesla. Although, in theory, it is possible to have a path that acts as a 'concentrator' and creates a higher flux density in an air-gap (say 5 Tesla), it is impractical. Why? The 'saturation flux density' $\boldsymbol{B}_{\text {sat }}$ of a typical path material - say soft iron - is about 1.5 Tesla. In fact, the material shows soft saturation above about 1 Tesla. This in turn means that if the flux density in any part of a magnetic path constructed with soft iron is larger than 1 Tesla, the path will show larger 'resistance' than expected and there will be some flux leakage. A practical rule is to design for 1.0 Tesla flux density. (For some applications, if a lower flux density is desired, this is okay; 1.0 Tesla is only an upper limit)

\section{Force (Electromagnetic actuation)}

If a conductor carrying a current $\boldsymbol{I}$ is placed in an air-gap with a magnetic field having flux density $\boldsymbol{B}_{\text {gap }}$, and if a length $\boldsymbol{L}$ of the conductor is exposed to the magnetic field (typically, the breadth of the pole shoe), then a force $\boldsymbol{F}_{\text {actuation }}$ is exerted on the conductor, which may be expressed as:

$$
\vec{F}_{\text {actuation }}=(\vec{B} \times \vec{L}) * I
$$

Limiting design factors are:

- If the conductor (actuated element) has a certain stroke, the pole shoe must be as broad ( $\left.\boldsymbol{b}_{\text {stroke }}\right)$, typically $50 \%$ more, to provide uniform actuation force over the entire stroke.

\footnotetext{
27 Saturation effects in core, leakage, etc. are not accounted for.
} 
- $\quad$ For the conductor (Actuated element) to move freely, the gap length $\boldsymbol{I}_{\text {gap }}$ must be at least as much as the thickness of the conductor, plus motion tolerances plus assembly tolerances. This restriction, when used with the 1.0 Tesla rule, gives an estimate of the required thickness of the magnet $\boldsymbol{I}_{\text {magnet }}$ (Typically 2 to 3 times as much as the gap) and the surface area of the magnet (typically 1.0 to 1.5 times the pole shoe area)

- $\quad$ As stated previously, a practical value for flux density is 1.0 Tesla. If a force $\boldsymbol{F}_{\text {actuation }}$ is desired in a conductor carrying a current $\boldsymbol{I}_{\text {actuation, }}$ then the parameter to vary is the actuation length $\boldsymbol{L}_{\text {actuation }}$ (not to be confused with the gap length $\boldsymbol{I}_{\text {gap }}$ ). Now, the airgap pole area is established as

$$
A_{\text {gap }}=L_{\text {actuation }} \cdot b_{\text {stroke }}
$$

- $\quad$ It is also possible to increase actuation force by increasing the current, $\boldsymbol{I}_{\text {actuation }}$. There are, however, two limitations to this

- A larger current often implies use of a thicker conductor. This may require a wider air-gap length, which will in turn reduce flux. (Not a relevant issue in the Coriolis actuator.)

- The current carrying conductor generates a field of its own. This is actually what causes the actuation. Its interaction with the gap field must be considered. (Not a relevant issue in the Coriolis actuator.)

- The actuation force on the conductor creates an equal and opposite reaction force, that acts upon the magnetic path (yoke). The yoke should hence, by design, be securely anchored.

\section{Pure torque}

In the Window-tube, in interest of exciting the rotational mode alone, a pure torque needs to be exerted. In principle, if we have the same current flowing through a tube (conductor), and if the tube is placed in a pair of equal (antiparallel) magnetic air-gaps, this should be achieved. To obtain identical air-gaps, the following points need to be considered:

1) The gaps should be mechanically identical. This means that the gap length, the actuation length and the stroke length must be congruent in each gap. The following errors may be expected:

a. If the gap length is not identical, no major difference in the flux density in the gap will be seen. This is due to the fact that, by design, the two gaps form a series magnetic circuit (see figure 1), and thus the flux through them is equal. A minor difference will, however, be seen, as a wider gap will have slightly different leakage pattern.

b. If the pole areas are unequal: (two cases)

i. If the stroke breadth $\boldsymbol{b}_{\text {stroke }}$ is different for each pole, it will substantially affect the flux densities [Tesla], as the same flux $\boldsymbol{\Phi}$ will create different flux densities $\boldsymbol{B}_{\text {gap } 1}$ and $\boldsymbol{B}_{\text {gap } 2}$.

ii. If the actuation length $\boldsymbol{L}_{\text {actuation }}$ is different, say smaller in gap 2, it will increase $\boldsymbol{B}_{\text {gap } 2}$ (same flux, smaller area) but as the active length is smaller, the two effects will cancel out, and the force will stay roughly constant

2) The pole faces should be aligned sideways. If they are not, the construction is no longer mirror-symmetric, and unequal leakages in each gap may result in an (unexpected) unequal B-field in the air-gaps

3) The stroke breadth should be designed appropriately. We assume that the B field is equal and uniform over the entire air-gap surface, and is perpendicular to it. In reality, there are fringe effects (curved field lines near the edges). If, during assembly, the tube is not exactly centered within the air-gap, then in too narrow a gap, an unequal pair of forces will be exerted (not desirable). 


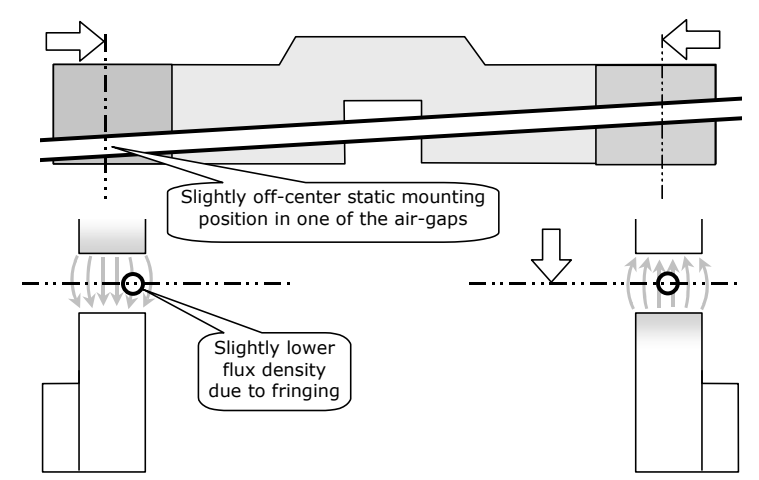

Figure B.2 An off-center section of tube sees lower flux density due to fringing

4) Parasitic magnetic paths and interfering fields: If other ferromagnetic objects (e.g. transformer cores) are designed to be near the static field yoke, they may provide an asymmetric leakage path for flux lines. This will cause unequal flux densities in each of the two air-gaps. As a general rule, static (immobile WRT the magnetic path) ferromagnetic components should be placed at a distance, at least $4 \cdot l_{\text {magnet }}$ away from the path, and preferably in a symmetric placement. A 'spacer' should be designed around the magnetic path so that other ferromagnetic objects (for example - bench tools, other instruments) are at a distance of at least $10 \cdot l_{\text {magnet }}$ away from the magnetic path. If this is not possible, the use of magnetic shielding should be considered. A magnetic shield is also necessary to block fields of other nearby magnets from affecting the air-gap flux.

Why is a pure torque essential? Our Coriolis tube has several eigenmodes of mechanical oscillation, of which two are of interest. One is what we call the actuation mode, in which the tube oscillates in such a way that a substantial section of the Coriolis tube sees a rotation motion in plane with the tube frame and perpendicular to the flow channel. This rotation, in presence of mass flow through the tube, causes a 'Coriolis force' that moves the tube, additionally, along the path of another mode, that we call the Coriolis mode. It is important to remember that the Coriolis force does not actually excite the Coriolis eigenmode, as it has a different frequency (specifically designed to be very different than the excitation eigenfrequency).

Now, the actuator is placed in such a way that actuation motion is a rotation ('twist') from its point of view and the Coriolis motion is a lateral 'swing'. If the actuator applies a pure torque (couple) on the Coriolis tube, it will cause motion only in the actuation-mode. If, however, it applies an unbalanced couple (force plus torque) it will also cause motion that will be confused as motion due to Coriolis force. This by itself is not a problem, as the measured Coriolis effect will merely show an offset. However, if the imbalance changes during use of the instrument, (due to one of several possible reasons) it will cause variation in the offset, which will be seen as a zero drift.

A typical example of this effect is when the viscous damping on the tube increases due to change in surrounding atmospheric conditions. In this case, to maintain constant oscillation amplitude, the actuator will have to apply a larger excitation torque, (say $110 \%)$. However, when the excitation current is increased to do this, the unwanted offset force will also increase to $110 \%$. Imagine that the initial offset corresponded to $100 \mathrm{~g} / \mathrm{h}$. (compensated - calibrated to zero). In the new situation, the offset will be $110 \mathrm{~g} / \mathrm{h}$, which, after compensation, will appear as an offset of $10 \mathrm{~g} / \mathrm{h}$. This is a severe error.

If on the other hand, the actuator applied a purer torque, the initial offset would, say, be only $1.0 \mathrm{~g} / \mathrm{h}$ (calibrated to zero). After the same atmospheric change, an offset of only 0.1 $\mathrm{g} / \mathrm{h}$ will be seen.

Some mechanisms that my cause errors of this type are:

- Varying air damping 
- Varying tube frequency (due to temperature or density; higher eigenfrequency will require larger actuation force)

- Variation in sensitivity of opto sensors

\section{B.2 Dimensioning of current inducing transformer}

To oscillate our Coriolis tube, we propose electromagnetic actuation. We need to have a current in a conductor (i.e. the tube itself) placed in a magnetic air-gap in order to generate a force on it. Direct injection of current into the tube is not desirable for two reasons:

- Stray currents flowing along the tube can cause unwanted actuation

- We may only use stainless steel for all places exposed to fluid. This makes electrical insulation impossible; an injected current can take paths other than that which would cause actuation (e.g. a shorter path through the chassis)

To target these shortcomings of direct current injection, we propose that the fluid carrying tube be designed as a secondary winding of a transformer. Applying excitation to a primary winding will induce a current in the secondary winding. Further, a "short circuit" through the chassis will function to complete the (secondary) circuit. An added advantage of this method is that the actuation current is induced in a contact-less fashion, thus without affecting the mechanical properties of the tube.

\section{Sizing}

From measurements, it has been determined (taking into account the Q-factor etc.) that we need roughly $14 \mathrm{~mA}$ peak current through the Coriolis tube (with typical dimensions of the stator magnet yoke) to sustain sufficient amplitude of oscillations. During startup, to rapidly get the instrument operational, a "startup current", about 5x larger, is needed. Thus the requirement on the transformer is that it should be able to induce $70 \mathrm{~mA}$ in the tube, at the tube eigenfrequency.

Further, it has been measured (and also calculated) that the Coriolis tube has an electrical resistance of roughly $0.6 \mathrm{ohm}$. Assuming purely resistive behavior, during startup, the Transformer core must induce about $42 \mathrm{mV}$ in the one-turn secondary circuit (the tube).

Operating, for example, at $170 \mathrm{~Hz}$, the transformer is required to induce $70 \mathrm{~mA}$ into a $0.6 \mathrm{ohm}$ secondary. It thus needs to induce $42 \mathrm{mV}$ in the secondary, i.e.

$$
v_{\text {secondary }}=42 \cdot 10^{-3} \cdot \sin 2 \pi \cdot 170 \cdot t
$$

Now,

$$
v=\frac{d \Phi}{d t}
$$

Or put differently,

$$
\Phi=\int v \cdot d t=\int 42 \cdot 10^{-3} \cdot \sin (2 \pi \cdot 170 \cdot t) \cdot d t=-\frac{42 \cdot 10^{-3}}{2 \pi \cdot 170} \cdot \cos (2 \pi \cdot 170 \cdot t)
$$

Thus the amplitude of the flux is

$$
\Phi_{\max }=\frac{42 \cdot 10^{-3}}{2 \pi \cdot 170}[\mathrm{~Wb}]
$$

Now, for a typical soft iron core, the saturation flux density is about 1 Tesla ([Wb/m^2]) So, for the required flux, so as not to exceed the maximum flux density (i.e. so as to not saturate the core), we need a transformer cross-section area of:

$$
A_{\min }=\frac{\Phi_{\max }}{B_{\max }}=\frac{42 \cdot 10^{-3}}{2 \pi \cdot 170} / 1 \approx 40 \cdot 10^{-6}\left[\mathrm{~m}^{2}\right]
$$




\section{Number of primary turns}

We need to induce a certain flux (40e-6 [Wb] peak) at a certain frequency $(170[\mathrm{~Hz}])$ in the transformer core. To do this we need a voltage waveform with certain amplitude. A pragmatic consideration is to choose this amplitude on basis of the available power supply. Using a fullbridge amplifier, it is possible to get a peak output voltage equal to the supply voltage. A $10 \mathrm{~V}$ DC supply is a convenient choice; thus a peak voltage of $10 \mathrm{~V}$ is available to induce flux. The peak voltage, the induced flux and the number of turns are, once again, related as

$$
v=N \frac{d \Phi}{d t}
$$

But the induced flux is sinusoidal in time, such that:

$$
\Phi=\Phi_{\max } \cdot \sin \left(2 \pi f_{\text {eig }} t\right)=40 \cdot 10^{-6} \cdot \sin (2 \pi \cdot 170 \cdot t)
$$

Thus

$$
\frac{d \Phi}{d t}=40 \cdot 10^{-6} \cdot 2 \pi \cdot 170 \cdot \cos (2 \pi \cdot 170 \cdot t)
$$

For a primary coil having $\boldsymbol{N}$ turns, this gives the peak amplitude of the voltage waveform as $v_{\max }=N \cdot 40 \cdot 10^{-6} \cdot 2 \pi \cdot 170=0.04 \cdot N$

But, $\mathrm{V}_{\max }$ must be less than $10 \mathrm{~V}$. Thus, the inequality

$0.04 \cdot N \leq 10$

i.e.

$N \leq 250$ must hold.

This is a good starting point for constructing the primary windings. 


\section{Appendix C - Transimpedance amplifiers}

\section{Transimpedance amplifier for occlusion measurement}

\section{Sensor with simple voltage amplifier}
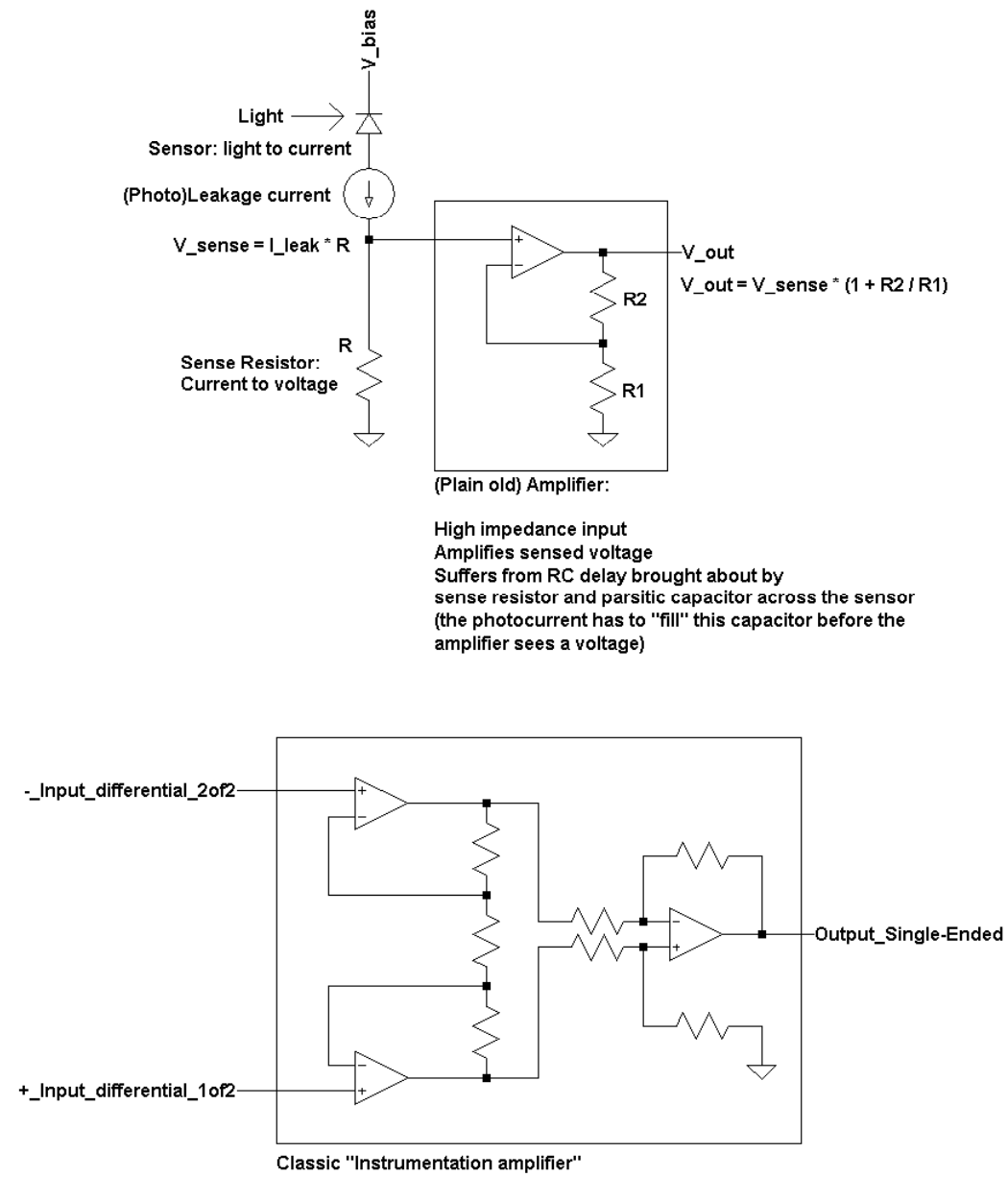

High input impedance, low offset, high gain, differential measurement Typically used with high impedance small-signal sensors e.g. bridges, load cells etc Increasing speed (reducing delays) is NOT its intended function

Figure C.1

Both illustrations show voltage amplifiers (gain is expressed as "Volts-per-Volt"). The first illustration is a simple op-amp based non-inverting amplifier. Many voltage-amplifier topologies are known; this is just one example. While the R-C delay is reduced owing to the use of a smaller ' $R$ ', it is still a finite value. The amplifier may also add delays of its own.

The second illustration is merely a modification to the first. It is commonly known as an instrumentation amplifier; used to sense a differential signal (from bridge sensors). This topology is used with matched input stages and is intended primarily to provide high common mode rejection. It does not provide significant speed advantage over the previous scheme. (Actually, this second scheme can be imagined as a pair of non-inverting amplifiers in mirror image, followed by a 'subtractor'.) This circuit is shown in the patent 275367 (figures 12 and 14) to amplify the output signal of the pickup coils (with low offset). 
Voltage amplifiers have high input impedances (i.e. the input current is forced constant (or zero)). They are suitable if the sensor is a voltage source or has a large inductance. Not being the case with our opto sensor, this conventional topology is not the best-suited solution for our opto sensors.

A different amplifier topology called "transimpedance amplifier" is commonly used in communications receivers (having optical receivers) to practically eliminate the effect of the parasitic capacitance. This is achieved by having a low impedance input instead of the conventional high impedance input. In other words, the input voltage of the amplifier is held (forced) constant; the current flowing in/out of the input is used for measurement purposes. Naturally, the gain is specified as Volts-per-Ampere (hence the name "trans-impedance"). An implementation schematic is shown below:

\section{Transimpedance amplifier:}

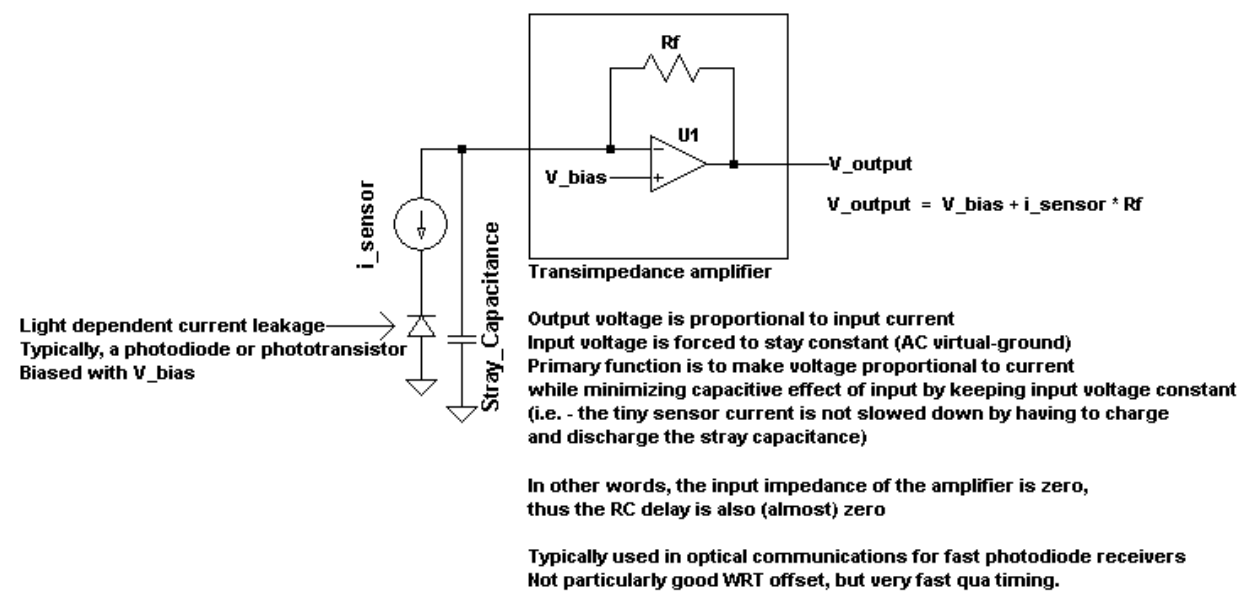

Figure C.2

The op-amp must drive its inverting input (and thus the top-side of the sensor) to a constant voltage equal to $U_{\text {bias }}$. The sensor leaks a variable current, proportional to incident light; the op-amp mirrors this by generating an output voltage such that an equal and opposite current is set up in the feedback resistor. The output voltage is then proportional to the photocurrent:

$$
U_{\text {output }}=U_{\text {bias }}+\left(R_{f} \cdot I_{\text {sensor }}\right)
$$

By choosing a fast op-amp, it is possible to reduce the original R-C delay by orders of magnitude. The "trick" here being that the voltage across the photo-sensor is held constant at V-bias; the parasitic capacitor is thus neither charged nor discharged, and thus is ineffective in causing delays. Another way of looking at it is to imagine that due to the low input resistance of the amplifier, the equivalent sense-resistor is zero; the R.C time constant is thus also zero (no delay). 


\section{Appendix D - Commercial Coriolis flowmeters}

Coriolis mass-flow meters - state of the art Listing of commercial instruments:

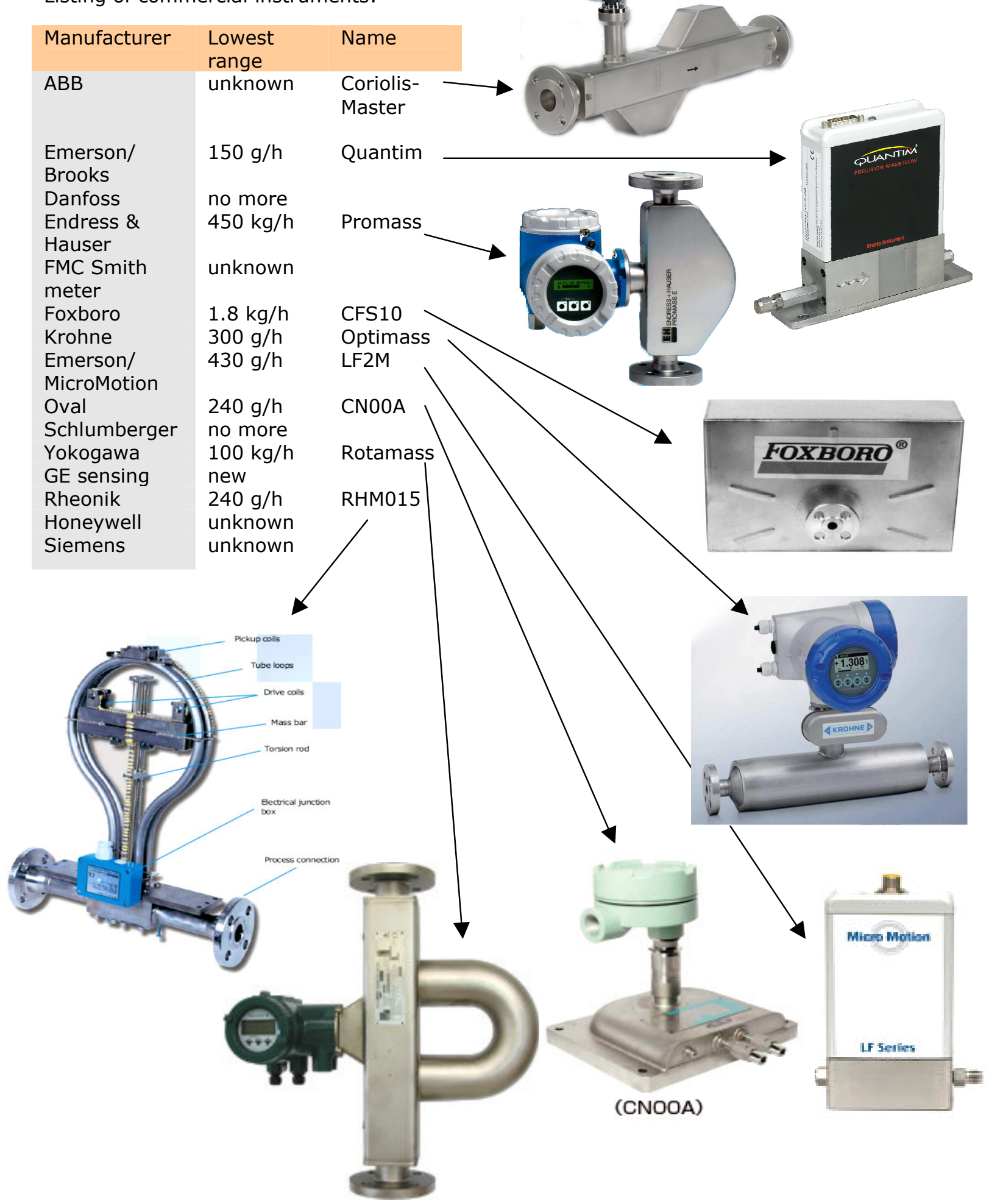




\section{References}

[1] Richard P. Feynman, Robert B. Leighton, Matthew Sands, The Feynman Lectures on Physics, The Definitive and Extended Edition, $2^{\text {nd }}$ edition, Addison-Wesley (2005), ISBN 0-8053-9045-6

[2] Stephen Hawking, A brief history of time, Bantam (1988)

[3] M.P. Koster, Constructieprincipes, PPI Uitgeverij (2005), ISBN 90-78249-01-3

[4] P.P.L. Regtien, F. van der Heijden, M.J. Korsten, W. Olthuis, Measurement Science for Engineers, Kogan Page Science (2004), ISBN 1903996589

[5] Simon Haykin, An Introduction to Analog and Digital Communication, Wiley (1989), ISBN 0-471-85978-8

[6] W. Wrigley, W.M. Hollister, W.G. Denhard, Gyroscopic Theory, Design, and Instrumentation, The M.I.T. Press (1969), SBN 262230372

[7] M.L. Meade, Lock-in amplifiers: principles and applications, IEE / Peter Peregrinus Ltd. (1983)

[8] Dennis Schipper (PhD Thesis), Mobile Autonomous Robot Twente, Twente University Press (2001), ISBN 9036516862

[9] Aditya Mehendale (Masters' thesis), Validation, Characterization and Performance Enhancements for a Low Capacity Coriolis Mass Flow Meter, University of Twente (2003)

[10] Marcel Katerberg (Masters' thesis), Coriolis Mass Flow Sensor and Controller, University of Twente (2004)

[11] MIL-STD-961E (1 August 2003). Paragraph 5.9.1

[12] Aditya Mehendale, P.P.L. Regtien, System design of low capacity Coriolis mass-flow meters, Conference proceedings, I3S 2005 - Juelich (2005)

[13] Poly-technisch zakboek (Nederlands), 49th print, Elsevier (2002), ISBN 90-6228-358-6

[14] Piezoelectric Ceramics Speciality Ferrites, Data handbook MA03, Philips components (1997)

[15] C.M. Lievers (Masters' thesis), Coriolis Mass-Flow Meters, University of Twente (2000)

[16] Peter H. Sydenham, Richard Thorn, Handbook of measuring system design, volume 3, Wiley (2005), ISBN 0-470-02143-8

[17] Lisa Bates, Timothy Beach and Maya Arnott, Determination of the temperature dependence of Young's modulus for stainless steel using a tuning fork, Vol. 18 \#1, The journal of undergraduate research in physics (1999)

\section{Literature}

[18] A. Belhadj, R. Cheesewright, C. Clark, The simulation of Coriolis meter response to pulsating flow using a general purpose F.E. code, Journal of fluids and structures, Vol. 14 , No. 5 (2000), pp 613-634

[19] Furio Cascetta, Effect of fluid pressure on Coriolis mass flowmeter's performance, ISA transactions 35 (1996) pp 365-370 
[20] R. Cheesewright, C. Clark, D. Bisset, Understanding the experimental response of Coriolis massflow meters to flow pulsations, Flow measurement and instrumentation 10 (1999) pp 207-215

[21] R. Cheesewright, C. Clark, D. Bisset, The identification of external factors which influence the calibration of Coriolis massflow meters, Flow measurement and instrumentation 11 (2000) pp 1-10

[22] Thierry Corman, Peter Enoksson, Kjell Noren, Goran stemme, A low-pressure encapsulated resonant fluid density sensor with feedback control electronics, Meas. Sci. Technol. 11 (2000) pp 205-211

[23] Thierry Corman, Peter Enoksson, Kjell Noren, Goran stemme, Novel burst technology for closed loop detection and excitation of resonant silicon sensors, $\mathrm{KTH}$, Sweden

[24] Peter Enoksson, Goran Stemme, Erik Stemme, A silicon resonant sensor structure for Coriolis mass-flow measurements, Journal of microelectromechanical systems, Vol. 6 , No. 2 (1997)

[25] John Hemp, Lynn Hendry, The weight vector theory of Coriolis mass flowmeters - Part 2. Boundary source of secondary vibration, Flow Meas. Instrum., Vol. 6, No. 4 (1995) pp 259-264

[26] Manus Henry, On-line compensation in a digital Coriolis mass flow meter, Flow measurement and instrumentation 12 (2001) pp 147-161

[27] Henry, Clarke, Archer, Bowles, Leahy, Liu, Vignos, Zhou, A self validating Coriolis massflow meter: an overview, Control engineering practice 8 (2000) pp 487-506

[28] K. Kolahi, Th. Gast, H. Rock, Coriolis mass flow measurement of gas under normal conditions, Flow measurement and instrumentation, Vol. 5(4) (1994) pp 275-283

[29] Tony Pankratz, Gary Pawlas, Improved mass flowrate measurement of gases using Coriolis mass flowmeters, SENSOR (1995), pp 103-108

[30] Ralf Storm, Kourosh Kolahi, Helmut Rock, Model based correction of Coriolis mass flowmeters, IEEE transactions on instrumentation and measurement, Vol. 51, No. 4 (2002)

[31] G. Sultan, J. Hemp, Modeling of the Coriolis mass flowmeter, Journal of Sound and Vibration, 132(3) (1989) pp 473-489

[32] Timo Veijola, Thierry Corman, Peter Enoksson, Goran Stemme, Dynamic simulation model for a vibrating fluid density sensor, Sensors and actuators 76 (1999) pp 213-224

[33] Yafan Zang, Srinivas Tadigadapa, Nader Najafi, A micromachined Coriolis-force based mass flowmeter for direct mass flow and fluid density measurement, Transducers (2001) pp 1460-1463

\section{Patents}

[34] Bergamini Giorgio, Campanale Nicola, Cocozza Onofrio, (US4895030) Process for the measurement of weight flow-rates and related devices, Nuovo Pignone (1990)

[35] Bose Tamal, Derby Howard Vincent, Levien Andrew Keith, Pankratz Anthony William, (US5734112) Method and apparatus for measuring pressure in a Coriolis mass flowmeter, Flowtec (1998)

[36] Cage Donald R, (US4768385) Parallel path Coriolis mass flow meter, Micro-motion (1988) 
[37] Carpenter Brent L, (US4777833) Ferromagnetic drive and velocity sensors for a Coriolis mass flow rate meter, Micro-motion (1988)

[38] Corwon Michael E, Oliver Randy L, (US5044208) Double-pigtail-shaped Coriolis-type mass flow rate meter, Neptune Meas. (1991)

[39] Corwon Michael E, Oliver Randy L, (US4852410) Omega-shaped, Coriolis-type mass flow rate meter, Schlumberger (1989)

[40] Cox Bruce M, Ho Morris D, (US4192184) Mass flowmeter, Halliburton, (1980)

[41] Cox Bruce M, Ho Morris D, (US4311054) Mass flowmeter with sensor gain control, Halliburton (1982)

[42] Cunningham Timothy J, (US6199022) Drive circuit modal filter for a vibrating tube flowmeter, Micro-motion (2001)

[43] Cunningham Timothy J, Shelley Stuart J, (US6092429) Driver for oscillating a vibrating conduit, Micro-motion (2000)

[44] Derby Howard V, Bose Tamal, Rajan Seeraman, (US5555190) Method and apparatus for adaptive line enhancement in Coriolis mass flow meter measurement, Micro-motion (1996)

[45] Gomi Shingo, Kitami Hirokazu, Endo Takashi, Matsuoka Kenichi, Ichinose Kimihiro, Futagawa Osamu, Kobayashi Seiji, Kobayashi Kazuhide, (US5796012) Error correcting Coriolis flowmeter, Oval (1998)

[46] Hussain Yousif A, Machacek Milos J, (US5048350) Electromagnetic driver and sensor, Foxboro (1991)

[47] Kalotay Paul Z, (US5400653) Coriolis effect meter using optical fiber sensors, Micromotion (1995)

[48] Kalotay Paul Z, (US5469748) Noise reduction filter system for a Coriolis flowmeter, Micro-motion (1995)

[49] Laursen Mogens Bech, Hansen Henning Max, Birker Bertel, (US6138517) Mass flowmeter with bidirectionally wound oscillation detector, Danfoss (2000)

[50] Mansfield William M, (US6226195) Circuitry for supplying a controlled signal to a drive system, Micro-motion (2001)

[51] Mattar Wade M, Thompson Duane T, Decarlo Joseph P, Hussain Yousif, Chitty Gordon W, (US4891991) Coriolis-type mass flowmeter, Foxboro (1990)

[52] Ollila Curtis John, Normen David Frederick, Lister Ernest Dale, (US5996225) Method for manufacturing a dual loop Coriolis effect mass flowmeter, Micro-motion (1999)

[53] Ollila Curtis John, Normen David Frederick, Lister Ernest Dale, (US6044715) Dual loop Coriolis effect mass flowmeter, Micro-motion (2000)

[54] Shelley Stuart J, Cunningham Timothy J, (US6230104) Combined pickoff and oscillatory driver for use in Coriolis flowmeters and method of operating the same, Micro-motion (2001)

[55] Stadler Dietmar, (US6073495) Measuring and operating circuit of a Coriolis-type mass flow meter, Flowtec (2000)

[56] Thompson Duane T, (US4911020) Coriolis-type mass flowmeter circuitry, Foxboro (1990)

[57] Thompson Duane T, (US5050439) Coriolis-type mass flowmeter circuitry, Foxboro (1991)

[58] Yokoi Toyoaki, Owada Hiroshi, (US5578764) Coriolis type mass flowmeter utilizing phase shifters for phase shifting of the output signals, Yokogawa (1996) 
[59] Yoshimura Hiroyuki, Kudo Takahiro, (US5869770) Coriolis type mass flowmeter having isolation form external vibration, Fuji Electric (1999)

[60] Yoshimura Hiroyuki, Kudo Takahiro, Kishiro Masami, (US5831178) Vibration type measuring instrument, Fuji Electric (1998)

[61] Yoshimura Hiroyuki, Morita Akira, (US5844408) Phase difference measuring apparatus for measuring phase difference between input signals, Fuji Electric (1998)

[62] Young Alan M, Blake E Ronald, (US4914956) Method and circuit for processing sensory input signals of the type obtained from Coriolis mass flow rate sensors and the like, Exac (1990) 


\section{Acknowledgements}

First and foremost, I thank "Aai" (mum) for being just that.

I began my journey in The Netherlands as a master's student in 2001, and now, seven years later, this proefschrift is ready, marking a major milestone. This journey was possible and enjoyable thanks to many people.

Rini, you have been my mentor for most of this journey, and for this I am deeply indebted. Your wisdom, knowledge and enormous patience, together with attention to detail without losing sight of "the big picture" has been phenomenal. Additionally, you have sacrificed much of your personal time - evenings and weekends - for the numerous reviews of this dissertation. Thank you!

The chance to carry out this PhD research - a major part of my journey - was made possible in the group of Prof. Regtien. Paul, I have learnt much about the art of measurement from you. Thank you for all the freedom you gave me in the last four years.

Most of the work I did in the past years has been on the premises of Demcon - initially in Hengelo and then Oldenzaal. The "Demcon family" - a group of talented and able technologyenthusiasts - made every day worth looking forward to. Several people at Demcon have collaborated in realizing the working Coriolis mass-flowmeter. Dennis, a big thank you! for giving me the opportunity to be a part of the Demcon family, and for giving me the much needed pep-talks to persevere to finish this dissertation. Peter, your know-how and multidisciplinary expertise will never cease to amaze me. A special thanks to my office-mates at Demcon: Jeroen H, Gino, Mark, Matthijs (get well soon!), Angela, Renee, Hernes and Wouter, and also to the people in the Coriolis project - Mark, Wouter, Tom, Reinier, Bas, Pieter, Martijn, Remco, Henry, Jeffrey, Ruud, Lucas, Ed K, Ed S, Joke, Rudy, Renee, Chris, Hernes, Angela and Jos, and the behind-the-scene heroes Joke, Arno, Carla, Petra, Mirjam, Mathijn and Elly. Lars - it has been a pleasure working with you on the microCoriolis project. Thanks also to Jan $L$ and Angela for feedback \& corrections with the dissertation and Marvin for the late evening discussions.

Marcel, your feedback on the instrument performance (much of chapter 6 ) has been invaluable. It has been great working and sparring with you - discussing things from Indian food to the formation of bubbles in ethanol ;). Thanks for all this and for the numerous reviews of this dissertation. Thanks also to the team-that-may-not-be-named - especially Ronald, Jan, Rob and Joost.

At the UT, thanks are due to people at the group where I was an AIO - Carla, Liesbeth, Joan, Alfred, and also Brenda, Ellen and Ila for their organizational help - from TAS forms to residence permits - and my fellow AIOs, especially Valer, Jianbo, Marcel G, Edwin \& Gijs. After the term of my PhD contract, I have been accepted as a researcher at the chair of mechanical automation and mechatronics. Thanks to Prof. Jonker for this opportunity and to Dannis and Martina for the organizational help.

My home in The Netherlands in these years has been Calslaan 7-3 "Maar-dan-niet". A big thanks to the folks (and ex-folks) at home - Alex, Andrea, Ben, Bouke, Douwe, Edwin, Gert, Jelmer, Mareike, Marjolein, Niels, Peter, Piet, Rama, Richard, Sjoerd, Steve, Vio, and of course, Mies, for making the journey a pleasure!

Unorthodox as it seems, I find it unfair not to do so - so here goes: A big thanks to team Blizzard, for making Starcraft/Broodwars - in my opinion the greatest game ever, and to Pepsi - my favorite source of caffeine and sugar into the late nights.

Aditya Mehendale

Enschede, 2008 


\section{About the author}

Aditya Mehendale was born on the 2nd of June 1976 in Pune, India. After finishing higher secondary education, he went on to graduate with distinction (Bachelor's degree in electronics engineering) from the University of Pune in 1998. Having done technical projects since age 12, he joined in partnership in the company "IDG product development", with friend and fellow alumnus Archis Bhave.

In 2001, he moved to the Netherlands to follow the master's program in Mechatronics at the Universiteit Twente in Enschede. In 2003, after completing the master's program with honors, he worked for a year as a researcher and subsequently began work towards a Ph.D., both under Prof. Paul Regtien, and in close collaboration with Demcon, initially in Hengelo, and then Oldenzaal.

Since June 2008, he works as a researcher in "Mechanical Automation and Mechatronics" group of Prof. Ben Jonker, at Demcon, in the field of MEMS based (micro) Coriolis devices. 